\title{
QUARTERLY TECHNICAL PROGRESS REPORT ON WATER REACTOR SAFETY PROGRAMS SPONSORED BY THE NUCLEAR REGULATORY COMMISSION'S DIVISION \\ OF REACTOR SAFETY RESEARCH OCTOBER-DECEMBER 1976
}

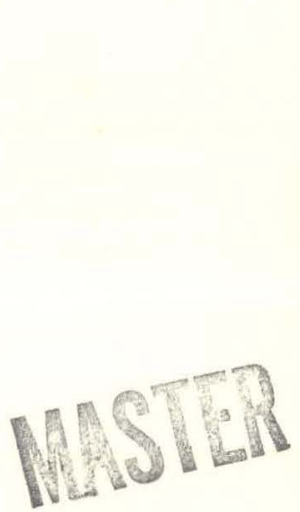

April 1977

EG\&G Idaho, Inc. 


\section{DISCLAIMER}

This report was prepared as an account of work sponsored by an agency of the United States Government. Neither the United States Government nor any agency Thereof, nor any of their employees, makes any warranty, express or implied, or assumes any legal liability or responsibility for the accuracy, completeness, or usefulness of any information, apparatus, product, or process disclosed, or represents that its use would not infringe privately owned rights. Reference herein to any specific commercial product, process, or service by trade name, trademark, manufacturer, or otherwise does not necessarily constitute or imply its endorsement, recommendation, or favoring by the United States Government or any agency thereof. The views and opinions of authors expressed herein do not necessarily state or reflect those of the United States Government or any agency thereof. 


\section{DISCLAIMER}

Portions of this document may be illegible in electronic image products. Images are produced from the best available original document. 
Printed in the United States of America Available from

National Technical Information Service

U.S. Department of Commerce

5285 Port Royal Road

Springfield, Virginia 22161

Price: Printed Copy $\$ 7.50$; Microfiche $\$ 3.00$

"The NRC will make available data tapes and operational computer codes on research programs dealing with postulated loss-of-coolant accidents in light water reactors. Persons requesting this information must reimburse the NRC contractors for their expenses in preparing copies of the data tapes and the operational computer codes. Requests should be submitted to the Research Applications Branch, Office of Nuclear Regulatory Research, Nuclear Regulatory Commission, Washington, D.C. 20555."

\section{NOTICE}

This report was prepared as an account of work sponsored by the United States Government. Neither the United States nor the Energy Research and Development Administration, nor the Nuclear Regulatory Commission, nor any of their employees, nor any of their contractors, subcontractors, or their employees, makes any warranty, express or implied, or assumes any legal liability or responsibility for the accuracy, completeness or usefulness of any information, apparatus, product or process disclosed, or represents that its use would not infringe privately owned rights. 
TREE-NUREG-1070

QUARTERLY TECHNICAL PROGRESS REPORT ON WATER REACTOR SAFETY PROGRAMS SPONSORED BY THE NUCLEAR REGULATORY COMMISSION'S DIVISION OF REACTOR SAFETY RESEARCH, OCTOBER - DECEMBER 1976
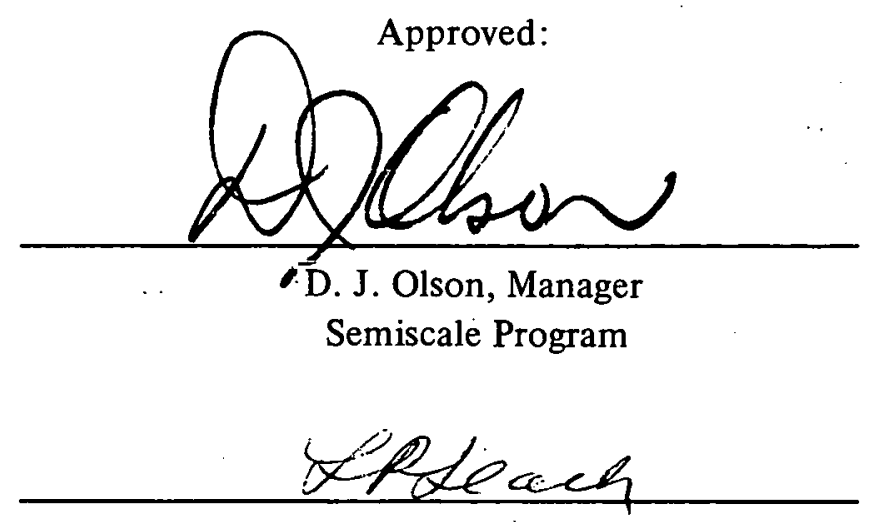

L. P. Leach, Acting Manager

LOFT Experimental Programs

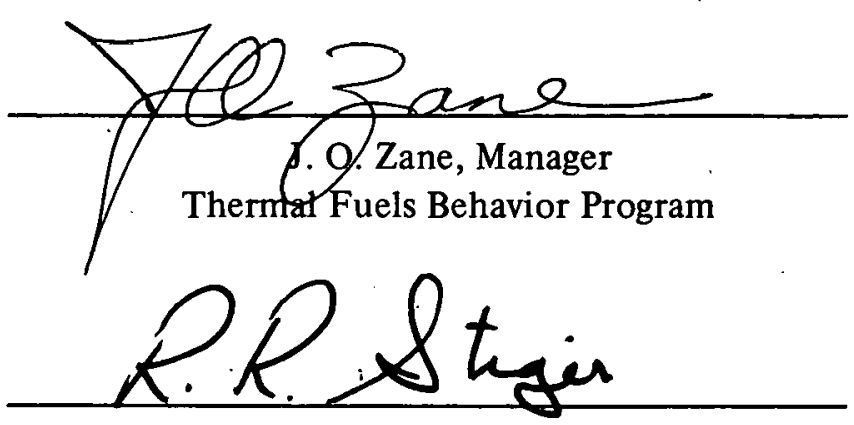

R. R. Stiver, Manager

Reactor Behavior Program

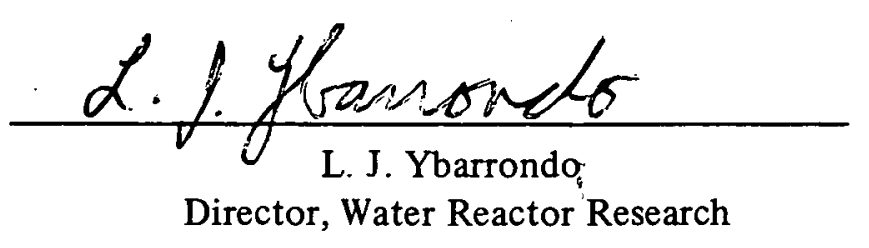

This report nares prepare sponsored by the United States Gocenumt of wo uk the United States nor the United States. Neither Research and Development Administration, nor any of subcontractors. of their employees contractors, warranty, express or implied, of assumes any legal
liability or responsbition for or usefulness of any information, apparat us, product process disclosed, or represents that its use would not
infringe privately owned rights.

4

$+$ 

NRC-2, -3 , and -4 Water Reactor Safety Research Systems Engineering Fuel Behavior Analysis. Development

\title{
QUARTERLY TECHNICAL PROGRESS REPORT ON WATER REACTOR SAFETY PROGRAMS SPONSORED BY THE NUCLEAR REGULATORY COMMISSION'S DIVISION OF REACTOR SAFETY RESEARCH, OCTOBER - DECEMBER 1976
}

EG\&G IDAHO, INC.

\section{IDAHO NATIONAL ENGINEERING LABORATORY IDAHO FALLS, IDAHO 83401}

\author{
Approved by L. J. Ybarrondo \\ Editor - J. B. Ferguson
}

April 1977

PREPARED FOR THE

U.S. NUCLEAR REGULATORY COMMISSION

AND

ENERGY RESEARCH AND DEVELOPMENT ADMINISTRATION

ITAAHOIOPERATIONS OFFICE

UNDER CONTRACT NO. EY-76-C-07-1570 


\begin{abstract}
Light water reactor safety research performed October through December 1976 is discussed. An analysis to determine the effect of emergency core coolant (ECC) injection location and pump speed on system response characteristics was performed. An analysis to evaluate the capability of commonly used critical heat flux (CHF) correlations to calculate the time of the first CHF in the Semiscale core during a loss of-coolant experiment (L.O.F.). was performed. A test program and study to determine the cffect thermocouples mounted on the outside fuel rod surfaces would have on the departure from nucleate boiling (DNB) phenomena in the LOFT core during steady state operation were completed. A correlation for use in predicting DNB lieat fluxes in the LOFT core was developed. Tests of an experimental transit time flowmeter were completed. A nuclear test was performed to obtain fuel rod behavior data from four PWR-type rods during film boiling operation representative of PWR conditions. Preliminary results from thc postirradiation examination of Test IE-1 fuel rods are given. Results of Irradiation Effects Tests IE- 2 and IE-3 are given. Gap Conductance Test GC 2-1 was performed to evaluate the effects of fuel density, initial gap width, and fill gas composition on the pellet-cladding gap conductance. Results of ongoing efforts with foreign experiment programs are reported.' The FRAP-T3 code was verified; the FRAP-S2 code was released to the Argonne Code Center for distribution to the nuclear industry. Efforts were directed toward finalizing the RELAP4/MOD6 code for simulating the reflood portion of a PWR LOCA. Development of the BEACON/MOD 1 code for use in investigating reactor containment phenomena continued. A study to investigate the sensitivity of the response surface uncertainty analysis technique to various unknuwns was completed. A study was made to compare the best-estimate and evaluation-model analyses of a PWR blowdown so the effect of differences necessitated by the conservatisms of a reactor licensing analysis could be assessed.
\end{abstract}




\section{PREFACE}

EG\&G Idaho, Inc. performs technical activites in the water reactor safety programs at the Idaho National Engineering Laboratory under the sponsorship of the U.S. Nuclear Regulatory Commission's Division of Reactor Safety Research. The current water reactor safety activities of EG\&G Idaho, Inc. are accomplished in four programs: the Semiscale, Program, the Loss-of-Fluid Test (LOFT) Experimental Program, the Thermal Fuels Behavior Program, and the Reactor Behavior Program.

The Semiscale Program consists of a continuing series of small-scale nonnuclear thermal-hydraulic experiments having as their primary purpose the generation of experiment data that can be applied to the development and verification of analytical models describing loss-of-coolant accident (LOCA) phenomena in water-cooled nuclear power plants. Emphasis is placed on acquiring system effects data from integral tests that characterize the most significant thermal-hydraulic phenomena likely to occur in the primary coolant system of a nuclear plant during the depressurization (blowdown) and emergency cooling phase of a LOCA. The current program of experiments employs the Semiscale Mod-1 test system, which has one intact loop with active components and a broken loop with passive components. These experiments include core reflood and emergency core cooling tests using an electrically heated 40-rod core.

The LOFT Experimental Program is a nuclear test program for providing test data to support: (a) assessment and improvement of the analytical methods utilized for predicting the behavior of pressurized water reactors (PWR) and LOCA conditions; (b) evaluation of the performance of PWR engineered safety features, particularly the emergency core cooling system; and (c) assessment of the quantitative margins of safety inherent in the performance of these safety features. The test program utilizes the LOFT facility an extensively instrumented 55-MW pressurized water reactor facility designed to conduct loss-of-coolant experiments (LOCEs). The test programs include a series of nonnuclear (without nuclear heat) LOCEs followed by series of low-power nuclear LOCEs and then a series of high-power nuclear LOCEs.

The Thermal Fuels Behavior Program is an integrated experimental and analytical program designed to provide information on the behavior of reactor fuels under normal, off-normal, and accident conditions. The experiment portion of the program is concentrated on testing of single fuel rods and fuel rod clusters under power-cooling mismatch, loss-of-coolant, and reactivity initiated accident conditions. These tests provide in-pile experiment data for the evaluation and verification of analytical models that are used to predict fuel behavior under reactor conditions spanning normal operation through severe hypothetical accidents. Data from this program provide a basis for improvement of the fuel models. 
The Reactor Behavior Program encompasses the analytical aspects of predicting the response of nuclear power reactors under normal, off-normal, and accident conditions. Program areas include loss-of-coolant accident, thermal-hydraulic code development, containment analysis development, and fuel behavior and code verification. The program produces computer codes for predicting the behavior of reactor systems, establishes data requirements for and performs verification of safety assessment codes, and maintains and improves computer codes to meet changing analytical and verification requirements.

A more detailed description of the four programs is presented in the quarterly technical report for January through March 1975, ANCR-1254. Later quarterly reports are ANCK-1262 (for April - June 1975), ANCR-1296 (for July - Seplember 1975), ANCR-NUREG-1301 (for October - December 1975), ANCR-NUREG-1315 (for January - March 1976), TREE-NUREG-1004 (for April - June 1976), and TREENUREG-1017 (for July - September 1976). Copies of the quarterly reports are available from the Technical Information Center, Energy Research and Development Administration, Oak Ridge, Tennessee. 


\section{SUMMARY}

Water reactor safety research activities performed by EG\&G Idaho, Inc. at the Idaho National Engineering Laboratory for October through December 1976 are reported.

In the Semiscale Program, an analysis was performed to determine the effect of emergency core coolant (ECC) injection, location, and pump speed on system response characteristics. The analysis was performed using the results of three double-ended cold leg break tests in the alternate ECC injection test series. In Test S-05-2, ECC was injected into the system at the intact loop pump suction by all ECC systems (accumulator, high pressure injection system, and low pressure injection system), and the pump speed was allowed to coast down from 2335 to $1500 \mathrm{rpm}$ in $10 \mathrm{sec}$. In Test S-05-2A, the high pressure system was disconnected, the low pressure system injection point was moved to the intact loop cold leg, and the pump speed was maintained throughout the test at the prerupture speed. Test S-05-2B was run at the same ECC injection conditions as Test S-05-2A, but the pump was allowed to coast down to a stop at $62 \mathrm{sec}$. Results of Test S-05-2 show that ECC injection into the pump suction leg can result in good core cooling during a loss-of-coolant accident (LOCA). However, excessive pump speed and a correspondingly high pumping capacity relative to the rate of ECC injection (as in Test S-05-2A) results in significantly degraded system thermal-hydraulic performance characteristics. Likewise, loss of pump power at the time of rupture, as simulated in Test S-05-2B, reduces the effectiveness of the ECC injection at the pump suction and may preclude cooling of the core.

Analysis was performed to evaluate the capability of commonly used critical heat flux (CHF) correlations to calculate the time of the first CHF in the Semiscale core during a LOCA. The correlations were evaluted by comparing calculated heat fluxes with mean fluxes from the test data. The conclusion reached, on the basis of evaluation of seven correlations, was that existing CHF correlations can be used to calculate the time of CHF in the Semiscale core during $200 \%$ cold leg break simulations.

A test program and study to determine the effect thermocouples mounted on the outside surface of fuel rods would have on the departure from nucleate boiling (DNB) phenomena in the LOFT core during steady state operation were completed. A correlation for use in predicting DNB heat fluxes in the LOFT core was developed from the test data obtained. It was found that the presence of the cladding thermocouples caused a reduction in the bundle steady state DNB heat flux. Tests of an experimental transit time flowmeter using thermocouples as sensors were completed. Tests indicate that thermocouple signals do not have enough correlated high frequency power to allow an acceptably low level of statistical error in transit time determination.

A nuclear test, Test PCM-4, was performed to obtain fuel rod behavior data from four PWR-type rods during film boiling operation representative of PWR conditions. Maximum cladding surface temperatures ranging from 810 to $1255 \mathrm{~K}$ were sought. "This temperature range includes the $\alpha$ phase and $\alpha+\beta$ phase of zirconium. The maximum measured cladding 
surface temperatures for the three instrumented rods were 930,1100 , and $1005 \mathrm{~K}$. The actual maximum cladding temperatures reached will be determined from detailed posttest metallurgical examination.

Preliminary results from the postirradiation examination of Test IE-1 fuel rods, mentioned in previous reports, show no evidence to suggest a more severe failure mode exists for irradiated fuel rods than for unirradiated fuel rods exposed to similiar transients, although the $\alpha-\mathrm{Zr}$ layer on the inside of the fuel rod cladding appeared to be thicker for the irradiated fuel rods.

Test IE-2 was performed with four PWR-type rods fabricated with fresh fuel and both unirradiated and previously irradiated cladding. No rod failures occurred as a result of pellet-cladding interaction or film boiling operation. Also, there was no evidence to suggest a difference in behavior between rods with fresh pellets clad with unirradiated or irradiated cladding.

Test IE-3 was performed with four PWR-type fuel rods which had been previously irradiated to 11,060 to $15,860 \mathrm{MWd} / \mathrm{t}$ in the Saxton Reactor. The power ramp following preconditioning produced pellet-cladding interaction but not rod failure. One rod apparently failed during reactor shutdown; this failure is being investigated.

Gap Conductance Test GC 2-1 was performed to evaluate the effects of fuel density, initial gap width, and fill gas composition on the pellet-cladding gap conductance. Preliminary results indicate that the test objectives were met and data were obtained from which on-line gap conductance values have been and will continue to be evaluated by steady state and power oscillation methods. Generally, differences between the steady state and power oscillation gap conductance values are large. The same trend for comparison between steady state, power oscillation, and predicted gap conductance values were observed for a previous test (Test GC 1-3).

Thermal fuel behavior development and evaluation efforts were concentrated in the areas of Halden fuel behavior research, light water reactor materials properties correlations, and postirradiation examination of power reactor fuel. Ongoing efforts with foreign experiment programs proceeded in the areas of reactivity initiated accident (RIA) and LOCA research. RIA research collaboration was carried out in meetings at the Japan Atomic Energy Research Institute (JAERI) in Tokai to review the Nuclear Safety Research Reactor and PBF experiment progress and plans. Technical liason with the Italian Government was initiated with discussion in Ispra and Rome concerning use of the ESSOR reactor, a test reactor located at the Ispra Research Center, for LOCA testing with bundled rods. Also, integration with German fuel behavior research work involved German-U.S.A. code comparisons.

In the area of fuel model development and verification, the FRAP-T3 code was verified. The FRAP-S2 code was transmitted to the Argonne Code Center for distribution to the nuclear industry. 
Analytical model development efforts were directed toward finalizing a version of the RELAP4 code for simulating the reflood portion of a PWR LOCA. This effort resulted in RELAP4/MOD6 Version 1, which contains all the MOD5 options plus the new reflood models. Code calculations for one blowdown and four reflood simulations showed reasonable agreement with the test data.

The containment analysis development efforts were primarily directed toward development of the BEACON code for use in investigating reactor containment phenomena. The latest version of the BEACON code, designated as BEACON/MOD1, contains all the capabilities developed to date. Test cases analyzed with the latest version indicate that the code is performing as desired and is a useful tool for analyzing containment systems.

A study to investigate the sensitivity of the response surface uncertainty analysis technique to various unknowns in the analysis was completed as a part of the code verification effort. A study to evaluate the RELAP4/MOD5 code with new data obtained from Oak Ridge National Laboratory and a sensitivity study to evaluate potential improvements in the model were also completed. A prediction of the first boiling water reactor blowdown/emergency core cooling test in the two-loop test apparatus at the General. Electric Company was made with the RELAP4/MOD5. EXP*P4/CE code, an experimental code very similar to RELAP4/MOD5 Update 2.

A study was made to compare best-estimate and evaluation-model analyses of a PWR blowdown, maintaining commonality when possible, so the effect of differences necessitated by the conservatisms of a reactor licensing analysis could be assessed. 


\section{CONTENTS}

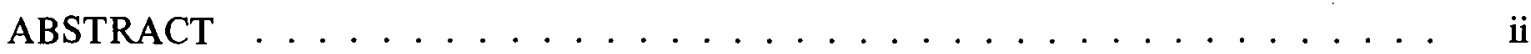

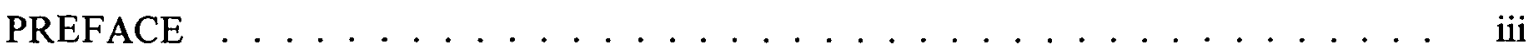

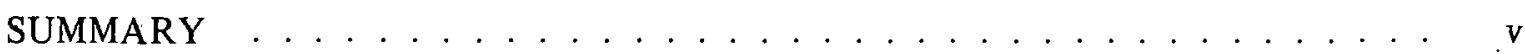

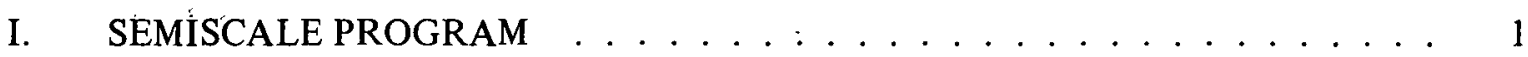

1. PRELIMINARY ANALYSIS OF THE EFFECTS

OF PUMP SPFFD ON SYSTFM RF.SPONSF. DIIRINR

I'UMY SUCLI'IUN EC' INJEC"I'IUN 'IESTS , . . . . . . . . . . . . . 1

1.1 System Behaviơ [uring Blowdown $\ldots \ldots \ldots \ldots$

1.2 System Behavior After Initiation of Intact

Loop ECC Injection . . . . . . . . . . . . . . . . 3

2. EVALUATION OF CHF CORRELATIONS FOR USE IN CALCULATING

TIME ÓF CHF IN SEMISCALE . . . . . . . . . . . . . . . 15

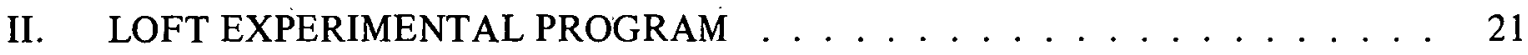

1. EFFECTS OF FUEL ROD SURFACE THERMOCOUPLES

ON STEADY STATE DEPARTURE FROM

NUCLEÁTE BOILING PHENOMENA . . . . . . . . . . . 21

1.1 DNB Test Program ................... 21

1.2 LOFT DNB Correlation .................. 25

2. TRANSIT TIME FLOWMETER DEVELOPMENT $\ldots \ldots . \ldots . \ldots . . \ldots 27$

III. THERMAL FUELS BEHAVIOR PROGRAM _ . . . . . . . . . . . . . . . . . 29

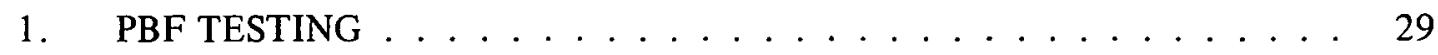

1.1 Power-Cooling-Mismatch (PCM) Test

PCM-4 Preliminary Results . . . . . . . . . . . . . . . . . 29

1.2 Irradiation Effects (IE) Test 1, Preliminary

Postirradiation Examination Results . . . . . . . . . . 38

1.3 Irradiation Etfects 'l'est 2, Preliminary Kesults . . . . . . . . . 48

1.4 Irradiation Effects Test 3, Preliminary Results . . . . . . . . . . . 57

1.5 Gap Conductance Test GC 2-1, Preliminary Results . . . . . . . . 63

2. THERMAL FUELS BEHAVIOR PROGRAM

DEVELOPMENT AND EVALUATION . . . . . . . . . . . . . 92 


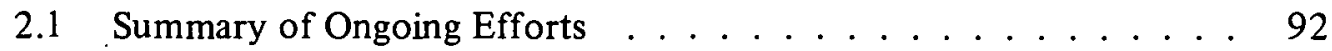

2.2 Cladding Creep Rate . . . . . . . . . . . . . . . . . . . . 94

2.3 Local Strains at Rupture . . . . . . . . . . . . . . . . 100

2.4 Axial Gas Flow Experiments: Comparison of Calculated and Measured Gas Flow Gaps . . . . . . . . . . . . . . 104

3. FUEL MODEL DEVELOPMENT AND VERIFICATION . . . . . . 111

3.1 Status of the Fuel Rod Failure Subçode, FRAIL . . . . . . 111

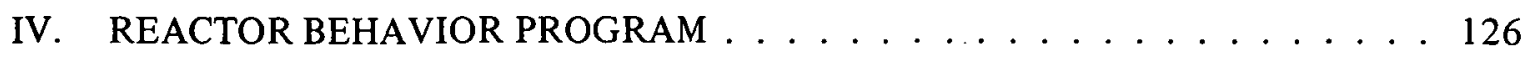

1. LOCA CODE DEVELOPMENT . . . . . . . . . . . 126

$1.1 \quad$ Moving Mesh Model . . . . . . . . . . . . . . . 127

1.2 Local Mass Flux Model . . . . . . . . . . . . . . . . . 127

1.3 Implicit and Explicit Entrainment Models . . . . . . . . . . . . 129

1.4 Deentrainment Model for Upper Plenum . . . . . . . . . . . . 133

1.5 Fallback Model for Core/Upper Plenum Junction . . . . . . . 134

1.6 Core Superheat Model for Reflood . . . . . . . . . . . 135

2. CONTAINMENT ANALYSIS DEVELOPMENT . . . . . . . . 139

2.1 Unequal Area Coupling Technique $\ldots \ldots \ldots 139$

2.2 Test Cases . . . . . . . . . . . . . . . . 140

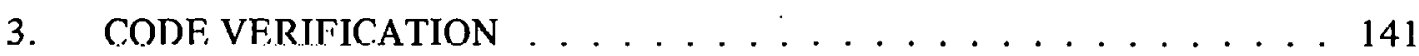

3.1 Sensitivity Study of the Response Surface

Method of Uncertainty Analysis . . . . . . . . . . . . . . . . 142

3.2. RFI.AP4/MnN5 Comparison with

ORNL PWR-BDHT Test $104 \ldots \ldots \ldots$. . . . . . . . 143

3.3 The First BWR-BD/ECC Test: A RELAP4 Prediction and

Comparison with a RELAP4 Analysis of the TLTA BDHT

Program Reference Test . . . . . . . . . . . . . 146

4. BEST ESTIMATE/EVALUATION MODEL COMPARISON $\ldots \ldots . .149$

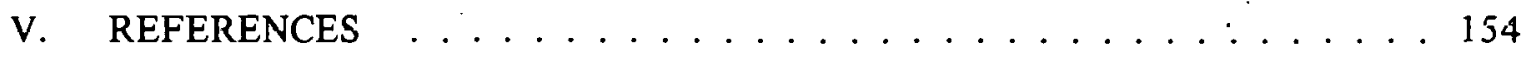

FIGURES

1. Semlscale Mod-1 system and instrumentation $\ldots \ldots \ldots \ldots$ 
2. Intact loop pump differential pressure (Test S-05-2) $\ldots \ldots \ldots$. . . . . 4

3. Intact loop pump differential pressure (Test S-05-2A) . . . . . . . 5

4. Intact loop pump differential pressure (Test S-05-2B) . . . . . . 5

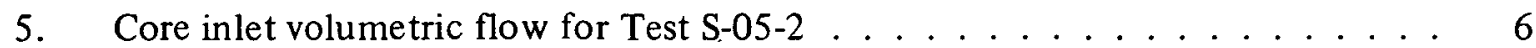

6. Core inlet volumetric flow for Test S-05-2A . . . . . . . . . 6

7. Core inlet volumetric flow for Test S-05-2B . . . . . . . . . . 7

8. Density at pump suction inlet (Test S-05-2A) f . . . . . . . . . . 7

9. Density at broken loop cold leg inlet nozzle (Test S-05-2) ... . . . . . 9

10. Density at broken loop cold leg inlet nozzle (Test S-05-2A) . . . . . . . . 9

11. Density at broken loop cold leg inlet nozzle (Test S-05-2B) . . . . . . . . 10

12. Downcomer collapsed liquid level . . . . . . . . . . . . . . . . 11

13. Rod temperature at the 13 -in. elevation . . . . . . . . . 12

14. Rod temperature at the 29 -in. elevation . . . . . . . . . . . . . 12

15. Rod temperature at the 33 -in. elevation $\ldots \ldots \ldots \ldots \ldots$

16. Calculated critical heat fluxes compared with the mean heat flux at the peak power step $(21$ to $31 \mathrm{in}.) \ldots \ldots . \ldots 16$

17. COBRA-IV-I calculated quality at axial core levels $\ldots \ldots \ldots 17$

18. Calculated critical heat tluxes compared with the mean

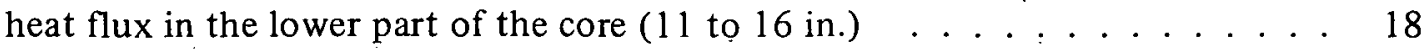

19. Calculated critical heat fluxes compared with the mean heat flux in the upper part of the core $(31$ to 36 in.) . . . . . . . . . 18

20. Variation in CHF from the General Electric Corporation correlation using hydraulics calculated from CUBKA-IV-I and the bounds in core heat transfer . . . . . . . . . . . . . . . . . . .

21. Variation in COBRA-IV-I calculated quality from the uncertain ty in core heat transfer rate 
22. Layout for bundle with external thermocouple simulators . . . . . . . . . 22

23. Layout for bundle without external thermocouple simulators . . . . . . . . 23

24. Comparison of measured and predicted critical heat flux (LOFT + COBRA-II) for LOFT bundles with external thermocouple simulators . . . . . . . . . . 25

25. Comparison of measured and predicted critical heat flux (LOFT + COBRA-II) for LOFT bundles without ex ternal thermocouple simulators . . . . . . . . . . 26

26. Histogram of ratio of measured-to-predicted critical heat fluxes for the LOFT correlation and source data . . . . . . . . . . . . . 27

27. Transit time standard deviation . . . . . . . . . . . . 28

28. Top view of PCM-4 fuel rod locations in the PBF in-pile tube ... . . . . . 30

29. Test PCM-4, Rod UTA-0014 $\left(0^{\circ}\right) \ldots \ldots \ldots$

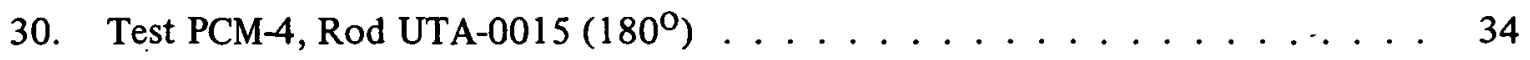

31. Test PCM-4, Rod UTA-0016 $\left(0^{\circ}\right) \ldots \ldots \ldots \ldots \ldots$

32. Test PCM-4, Rod A-0017 $\left(0^{\circ}\right) \ldots \ldots \ldots$

33. Predicted and measured cladding surface temperature versus fuel rod local power for steady state phase of Test PCM-4 . . . . . . . . . . . . . 39

34. Predicted and measured fuel centerline temperature versus fuel rod local power for steady state phase of Test PCM-4 . . . . . . . . . . . . . . . 40

35. Predicted and measured cladding elongation versus fuel rod average power for steady state phase of Test PCM-4 . . . . . . . . . . . . . . . 41

36. Posttest view of Rods IE-007, IE-008, IE-009, and IE-010 _ . . . . . . . . 42

37. Posttest diameter measurements on Test IE-1 irradiated rods . . . . . . . 44

38. Film boiling on Rod IE-007, which resulted in heavy surface oxidation, fuel melting, and fuel relocation . . . . . . . . . . . . . . 45

39. Metallography from Rod IE-009 . . . . . . . . . . . . . . . . 46

40, Highly embrittled cladding from Rod IE-007 . . . . . . . . . . . . . 47 
41. Isolated area on Rod IE-009 where cladding had collapsed into region of fuel relocation and fuel had impinged on the inside surface of the cladding . . . . . . . . . . . . . . . . 49

42. Nominal operating conditions during Test IE-2 . . . . . . . . . . 51

43. Fuel rod power for Rod IE-011 versus SPND current for Test IE-2 . . . . . . 52

44. Fuel rod power for Rod IE-2 versus SPND current for Test IE-2 $\quad \ldots \ldots$. . . . . 53

45. Fuel rod power for Rod IE-013 versus SPND current for Test IE- $2 \ldots \ldots$

46. Fuel rod power for Rod IE-U14 versus SPND current for 'l'est IE-2 $\ldots \ldots \ldots$

$4 \%$ Kod IE-U1l strip chart data showing traces during flow reduçtion . . . . . . . 56

48. Rod IE-013 strip chart data showing traces during flow reduction . . . . . . . . 57

49. Rod IE-014 strip chart data showing traces during flow reduction . . . . . . . 58

50. Predicted and measured fuel centerline temperatures versus local fuel rod power for all four rods during the preconditioning and high-power portions of the test . . . . . . . . . . . . . . 59

51. Nominal operating conditions during Test IE-3 f . . . . . . . . . . 60

52. Rod IE-018 strip chart showing traces during flow reduction for internal pressure, cladding elongation, cladding surface temperatures and flow rate $\ldots \ldots \ldots \ldots . \ldots . \ldots 2$

53. Diagram of relative azimuthal and axial locations of thermocouples and orientation of test fuel rods in $4 \mathrm{X}$ hardware for Test GC 2-1 . . . . . . . . . 66

54. Calculated cladding surface temperature oscillation for $\pm 20 \%$ amplitude, $20 \mathrm{~s} /$ cycle power oscillations at a nominal power density of $46 \mathrm{~kW} / \mathrm{m} \ldots \ldots \ldots \ldots 8$

55. Calculated gap thickness variations during $\pm 20 \%$ amplitude, $20 \mathrm{~s} /$ cycle power oscillations at a nominal power density of $46 \mathrm{~kW} / \mathrm{m} \ldots \ldots 8$

56. Calculated cladding surface temperature oscillations for $\pm 20 \%$ amplitude, $20 \mathrm{~s} /$ cycle power oscillations at a nominal power density of $52.5 \mathrm{~kW} / \mathrm{m} \ldots \ldots \ldots . \ldots . \ldots 69$

57. Calculated gap thickness variations during $\pm 20 \%$ amplitude, $20 \mathrm{~s} /$ cycle power oscillations at a nominal power density of $52.5 \mathrm{~kW} / \mathrm{m} \ldots . . .69$ 
58. Test fuel rod peak power density as a function of SPND 3 current for Test GC 2-1

59. Representative comparison of Rod GC 503 core power oscillations with cladding surface temperature oscillations at $240^{\circ}$ orientation with $\pm 20 \%$ amplitude, $20 \mathrm{~s} /$ cycle oscillations and $2.7 \mathrm{MW}$ core power . . . . . .

60. Representative comparison of Rod GC 504 core power oscillations with cladding surface temperature oscillations at $240^{\circ}$ orientation with $\pm 20 \%$ amplitude, 260 s/cycle oscillations and $2.7 \mathrm{MW}$ core power . . . . .

61. Comparison of Rod GC 504 cladding surface temperature oscillations at $0^{\circ}$ orientation at power density of $44 \mathrm{~kW} / \mathrm{m}$ with Rod GC 501 cladding surface temperature oscillations at $240^{\circ}$ orientation at power density of $48 \mathrm{~kW} / \mathrm{m}$ with $\pm 20 \%$ amplitude, $60 \mathrm{~s} /$ cycle

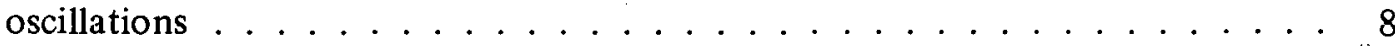

62. Comparison of Rod GC 501 cladding temperature oscillations at $240^{\circ}$ orientation at power density of $48 \mathrm{~kW} / \mathrm{m}$ with $\mathrm{PBF}$ power oscillations with $\pm 20 \%$ amplitude, 60 s/cycle oscillations $\ldots . . . . .$.

63. Comparison of Rod GC 504 cladding temperature oscillations at $0^{\circ}$ orientation at power density of $48 \mathrm{~kW} / \mathrm{m}$ with $\mathrm{PBF}$ power oscillations with $\pm 15 \%$ amplitude, $60 \mathrm{~s} /$ cycle oscillations

64. Comparison of Rod GC 504 cladding temperature oscillations at 0 and $240^{\circ}$ orientation at power density of $48 \mathrm{~kW} / \mathrm{m}$ with $\pm 15 \%$ amplitude, $60 \mathrm{~s} /$ cycle oscillations . . . . . . . . . . . .

05. Comparison of Rod GC 501 cladding temperature oscillations at $240^{\circ}$ orientation at power density of $52 \mathrm{~kW} / \mathrm{m}$ with core power oscillations with $\pm 15 \%$ amplitude, $60 \mathrm{~s} /$ cycle oscillations $\ldots \ldots . .$.

66. . Comparison of Rod GC 504 cladding temperature oscillations at $0^{\circ}$ orientation at power density of $48 \mathrm{~kW} / \mathrm{m}$ with core pơwer oscillations with $\pm 15 \%$ amplitude, 20 s/cycle oscillations

67. Fuel centerline temperature as a function of test rod power density for Rod GC 501 during Test GC 2-1 . . . . . . . . . . . . . 
68. Fuel centerline temperature as a function of test rod power density for Rod GC 502 during Test GC 2-1 . . . . . . . . . . . . . . . 86

69. Fuel centerline temperature as a function of test rod power density for Rod GC 503 during Test GC $2-1 \ldots \ldots$. . . . . . . . . . 87

70. Fuel centerline temperature as a function of test rod power density for Rod GC 504 during Test GC 2-1 f . . . . . . . . . . . . . . 88

71. Comparison of measured and predicted gap conductance values for Rod GC 501 during Test GC2-1 . . . . . . . . . . . . . . . . . . . . . . . . 89

72. Comparison of measurcd and predicted gap conductance values for Rod GC 502 during Test GC $2-1$. . . . . . . . . . . . . . . . . . . . . . . 89

73. Comparison of measured and predicted gap conductance values for Rod GC 503 during Test GC $2-1$ and GC 1-3 . . . . . . . . . . . . . 90

74. Comparison of measured and predicted gap conductance values for Rod GC 504 during Test GC 2-1 . . . . . . . . . . . . . . . . . . . 91

75. Comparison of the predictions of the cladding creep rate equation with the Saxton $[2,3,4]$ data . . . . . . . . . . . . . . 99

76. Comparison of the Ibrahim's ${ }^{[5]}$ pressure tube data with the prediction of the cladding creep rate equation . . . . . . . . . . 101

77. Location of the center of curvature of the side of the tube away from the burst region ..................... 103

78. Components of true local strain at rupture . . . . . . . . . . 106

79. Typical fuel crack pattern and fuel-to-cladding gap $\ldots \ldots \ldots$. . . . . 108

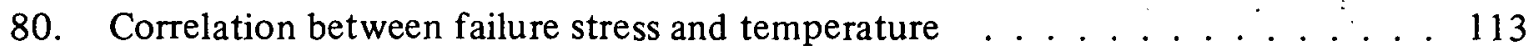

81. Comparison of empirical and assumed distribution of fallure stress . . . . 115

82. FRAIL prediction of pellet-cladding interaction failures . . . . . . . . 116

83. Correlation between stress and temperature at failure $\ldots \ldots \ldots \ldots$

84. Flow blockage correlation ..................... 120

85. Constant stress sample problem . . . . . . . . . . . . . 123 
86. Increasing temperature and stress sample problem . . . . . . . . . . . 124

87. Temperature reversal sample problem . . . . . . . . . . . . 125

88. Instantaneous moving mesh partitioning of coarse heat slab conductors . . . . 128

89. Physical description of entrainment within core volume using modified bubble rise approach . . . . . . . . . . . . . . . . 130

90. Configuration for test case .................... . . 140

91. Steady state pressure and velocity for test case . . . . . . . . . . 141

92. Effect on peak cladding temperature density caused by changing input uncertainty densities for discharge coefficient for vessel side of inlet pipe break and fuel gap conductivity from uniform to Gaussian . . . . . . . . . 143

93. Effect on peak cladding temperature density by changing standard deviations of input uncertainty densities . . . . . . . . . . . . . . 144

94. Comparison of calculated and measured cladding temperatures at hot spot . . . . . . . . . . . . . . . . . . . . 145

95. Cladding temperature at hot spot . . . . . . . . . . . 145

96. Comparison of steam dome pressure in TLTA-1 $7 \times 7$ bundle and TLTA-2 $8 \times 8$ bundle cases . . . . . . . . . . . . 147

97. Comparison of core inlet mass flow in TLTA-1 $7 \times 7$ bundle and TLTA-2 $8 \times 8$ bundle cases . . . . . . . . . . . . . . 147

98. Comparison of rod temperatures of TLTA-1 $7 \times 7$ bundle and TLTA-2 $8 \times 8$ bundle cases . . . . . . . . . . . . . . . 148

99. Analy tical models and analysis process . . . . . . . . . . . 151

100. Comparison of hot spot cladding temperature, evaluation model versus best estimates

\section{TABLES}

I. Rod Bundle Characteristics ................... 
II. Summary of Experiment Results During DNB

Testing for Test PCM-4 . . . . . . . . . . . . . . . 32

III. Film Boiling Zone on IE-1 Rods . . . . . . . . . . . . . . . 43

IV. Measurement Summary Made of Fuel Rods During Film Boiling . . . . . . . 55

V. Nominal Design Characteristics of BWR-Design Test Fuel

Rods for Gap Conductance Test GC 2-1 . . . . . . . . . . . . . . . . 65

VI. Oscillation Conditions During Power Oscillation

Pultiun of Test GC $2-1 \ldots \ldots \ldots 7$

VII. Preliminary On-Line Steady State Data From Gap

Conductance Test GC 2-1 $(\operatorname{Rod}$ GC 501) $\ldots \ldots$. . . . . . . . . . . 72

VIII. Preliminary On-Line Steady State Data From Gap

Conductance Test GC 2-1 (Rod GC 502) . . . . . . . . . . . . . . . . 73

IX. Preliminary On-Line Steady State Data From Gap

Conductance Test GC 2-1 (Rod GC 503) : . . . . . . . . . . . . . . . . . 74

X. Preliminary On-Line Steady State Data From Gap

Conductance Test GC $2-1(\operatorname{Rod}$ GC 504$) \ldots \ldots . \ldots 75$

XI. Preliminary Power Calibration Data From Test GC 2-1 . . . . . . . . . . 76

XII. Preliminary Steady State Gap Conductance Values From Test GC 2-1 . . . . 78

XIII. Preliminary Power Oscillation Data From Gap Conductance Test GC 2-1, Test Rod GC 501 (cladding thermocouple at $240^{\circ}, 0.452 \mathrm{~m}$ above bottom of fuel stack $\ldots \ldots \ldots \ldots$. . . . . . . . . . . . . . . . . . . . .

XIV. Preliminary Power Oscillation Data From Gap Conductance Test GC 2-1, Test Rod GC 502 (cladding thermocouple at $240^{\circ}, 0.452 \mathrm{~m}$ above bottom of fuel stack) . . . . . . . . . . . . . . . . .

XV. Preliminary Power Oscillation Data From Gap Conductance Test GC 2-1, Test Rod GC 503 (cladding thermocouple at $240^{\circ}, 0.452 \mathrm{~m}$ above bottom of fuel stack)

XVI. Preliminary Power Oscillation Data From Gap Conductance

Test CS 2-1, Test Rod 504 (cladding thermocouples at $240^{\circ}$,

$0.452 \mathrm{~m}$ above bottom of fuel stack

XVII. Saxton Rods Creep Data At 9750 Hours . . . . . . . . . . . . . . . . . 96 
XVIII. Ibrahim Pressure Tube Creep Data at 10,000 Hours . . . . . . . . . . . . 96

XIX. Components of Local True Strain . . . . . . . . . . . . 105

XX. Comparison of Calculated and Measured Effective

Annular Gaps in Rod $\mathrm{K}-4$. . . . . . . . . . . . . . . . 110

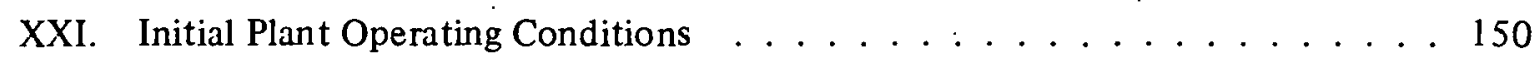

XXII. Assumption for LOCA Sequence . . . . . . . . . . . . . . 151 


\title{
QUARTERLY TECHNICAL PROGRESS REPORT ON WATER REACTOR SAFETY
}

\author{
PROGRAMS SPONSORED BY THE NUCLEAR REGULATORY COMMISSION'S
}

DIVISION OF REACTOR SAFETY RESEARCH, OCTOBER-DECEMBER 1976

\section{SEMISCALE PROGRAM}

D. J. Olson, Program Manager.

Results of three emergency core coolant (ECC) injection tests in which coolant was injected into the pump suction leg were analyzed to determine the effect of pump suction ECC injection and varied pump speed on the response of the Semiscale Mod-1 system. The relation between ECC injection location and pump speed is discussed in Section I-1.

The applicability to Semiscale of commonly used critical heat flux (CHF) correlations was evaluated. Section I-2 presents conclusions from this evaluation.

\section{PRELIMINARY ANALYSIS OF THE EFFECTS OF PUMP SPEED ON}

\section{SYSTEM RESPONSE DURING PUMP SUCTION ECC INJECTION TESTS}

\section{E. A. Harvego}

Three 200\% double-ended cold leg break tests (Tests S-05-2, S-05-2A, and S-05-2B) employing pump suction ECC injection were conducted in the Semiscale system as part of the alternate ECC injection test series. The principal objective of these tests was to assess the effect of pump speed on system response characteristics when ECC was injected into the pump suction leg. The following sections emphasize those areas of system behavior that were significantly affected by differences in pump operating characteristics. Also, to facilitate a complete discussion of thermal-hydraulic phenomena associated with ECC injection into the pump suction, significant results are compared with those from the baseline ECC injection test (Test S-04-6), which utilized cold leg ECC injection. The results of the tests are presented with respect to system behavior during blowdown and after initiation of intact loop accumulator injection.

The principal variable evaluated in the tests was pump speed. In Test S-05-2, the pump coasted down from an initial prerupture speed of approximately $2335 \mathrm{rpm}$ to a speed of $1500 \mathrm{rpm} 10 \mathrm{sec}$ after rupture and maintained that speed for the remainder of the test. To evaluate the effect of increased pump speed on system response, Test S-05-2A was run with the pump speed maintained at its initial prerupture value (approximately $2345 \mathrm{rpm}$ ) throughout the test. In Test S-05-2B, the pump was allowed to coast down from its prerupture speed to a stop approximately $62 \mathrm{sec}$ after rupture. The pump coastdown 
characteristic for Test S-05-2B was representative of the pump coastdown calculated for a pressurized water reactor (PWR) with pump suction injection and loss of pump power at the time of rupture. However, to ensure that the pump speed during Test S-05-2B was conservatively low, the coastdown characteristics were controlled so the pump speed would be $20 \%$ lower than that calculated for a PWR at any point in the coastdown.

For Test S-05-2, all intact loop ECC injection systems, which included the high pressure injection system (HPIS), the accumulator, and the low pressurc injection system (LPIS), were connected directly to the pump suction leg. For Tests S-05-2A and S-05-2B, the high pressure injection system was disconnected, and the low pressure injection system injection location was moved to the intact loop cold leg injection location.

In each of thc tcsts, after intact loop accumulator water was completely discharged, the nitrogen gas in the accumulator was allowed to discharge Into the purnp suction leg fur periods ranging from 25 to $28 \mathrm{sec}$.

The system and instrumentation used in the tests are shown in Figure 1. References 1 through 3 describe the system and instrumentation more extensively and present the data oblained.

The system conditions prior to initiation of injection of ECC by the accumulator are examined to determine whether differences in the system initial blowdown response affected system behavior during ECC injection. The system behavior during ECC injection is then examined to determine effects directly attributable to ECC injection and pump speed.

\subsection{System Behavior During Blowdown}

Comparizon of hoator rod temperatures for the three tesss indirated that: some variations occurred in rod peak temperatures and rod hot spot locations during initial blowdown. In Tests S-05-2 and S-05-2A, the rod hot spot occurred at the 29-in. elevation and the peak temperatures, reached $9 \mathrm{sec}$ after rupture, were $1475^{\circ} \mathrm{F}$ and $1510^{\circ} \mathrm{F}$, respectively. In Test S-05-2B, the measured peak blowdown temperature at the 29-in. elevation was only $1390^{\circ} \mathrm{F}$. As a result of this locally lower temperature at the 29-in. elevation, the core hot spot occurred at the 21 -in. elevation where the peak temperature, reached $9 \mathrm{sec}$ atter rupture, was $146^{\circ} \mathrm{U}^{\circ} \mathrm{F}$.

The reason for the difference in location of the rod hot spot in Test $\mathrm{S}-\mathrm{U} 5-2 \mathrm{~B}$ is not apparent and requires further analysis. Examination of the delayed deparlure lrom nucleate boiling and rewet characteristics of the three tests indicated behavior similar to that observed in Test S-04-6 ${ }^{[4]}$. Although some variations in heater rod temperatures between the three pump suction injection tests were apparent and may have contributed to minor variations in rod quench times in the lower third of the core (discussed in the following section), those differences were judged not sufficient to affect the core-wide thermal response significantly following the initial blowdown.

Comparisons of the system pressure, flow rates, and densities indicated that the system conditions at the initiation of pump suction ECC injection were nearly identical for 


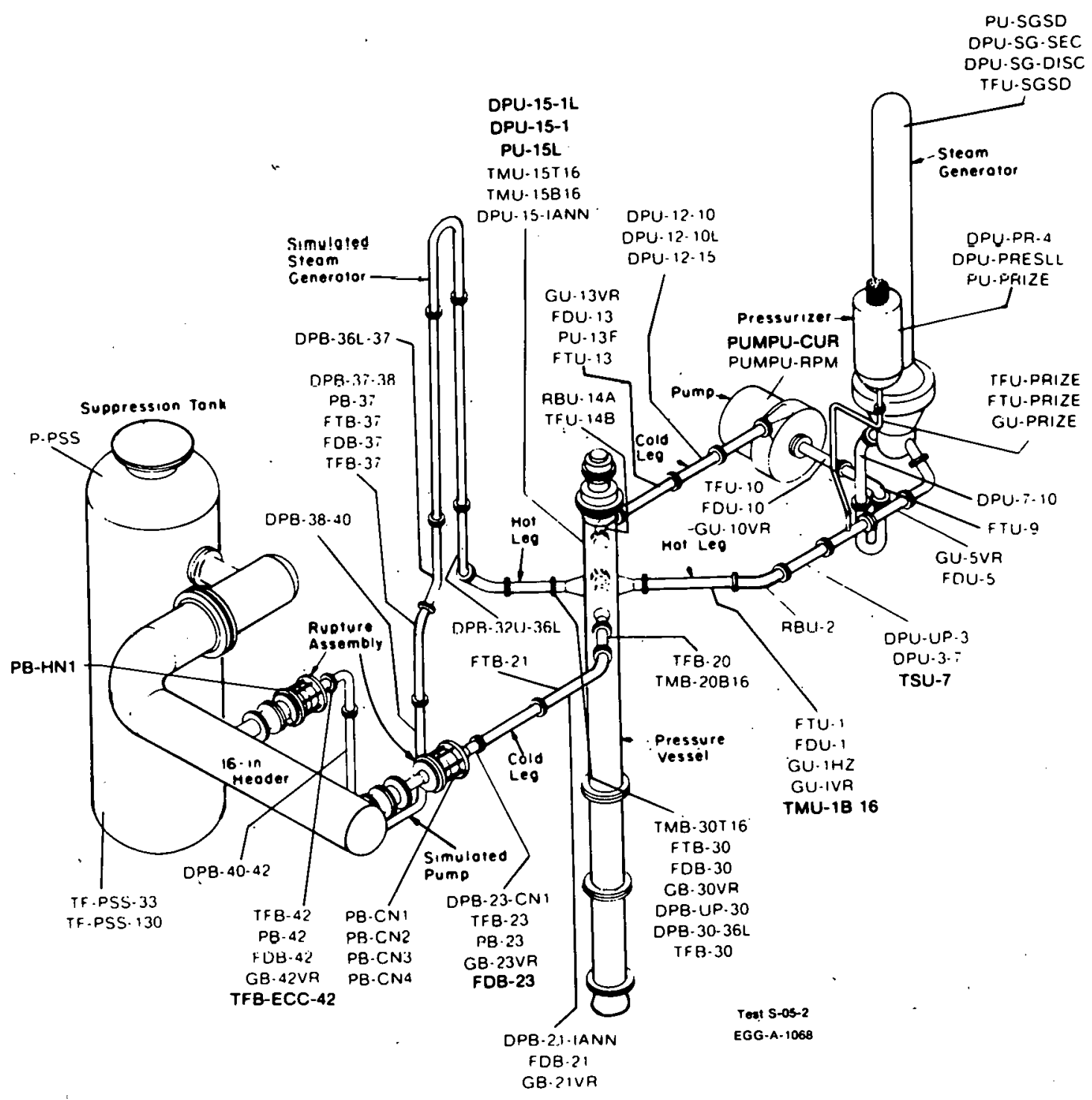

Fig. 1 Semiscale Mod-1 system and instrumentation.

Tests S-05-2, S-05-2A, and S-05-2B. The system conditions prior to ECC injection for the three pump suction injection tests were also nearly identical to those of the baseline test, Test S-04-6. Therefore, the conclusion reached was that the differences between Tests S-04-6, S-05-2, S-05-2A, and S-05-2B with respect to core and system response following the initiation of ECC injection are due primarily to the differences in pump operating characteristics and to differences in the ECC injection locations.

\subsection{System Behavior After Initiation of Intact Loop ECC Injection}

In all three tests, the system response characteristics were significantly affected by the differential pressure developed by the intact loop pump shortly after intact loop pump suction ECC injection was initiated. A delay time of 4 to $7 \mathrm{sec}$ between the start of ECC 
pump suction injection and the development of a substantial intact loop pump differential pressure in each of the tests, as shown in Figures 2, 3, and 4, is attributed to the time required to fill the pump suction leg. During this delay, no significant effect of ECC injection on system performance was observed. When the pump suction leg filled with ECC, the increased density of fluid at the pump inlet resulted in an increase in the developed pump differential pressure, and significant positive core and intact loop flow was observed in each of the pump suction injection tests. Figures 5, 6, and 7 show the initial positive surge in flow that developed at the core inlet when the initial increase occurred in the differential pressure across the pump.

The three tests together demonstrated that the system performance was dramatically affected by a relationship between pump speed and pump suction injection rate. The relationship was most apparent in Test S-05-2A (Figure 3). Large oscillations occurred in the differential pressure developed by the pump during this test. In 'lest $\mathrm{S}-05-2 \mathrm{~A}$, the pump was operated at full speed for the entire test. At this pump speed, the pump flow capacity was greater than the ECC injection rate, resulting in rapid depletion of water from the pump suction leg. The pressure differential developed by the pump then dropped until the pump suction leg refilled with ECC. When the pump pressure differential was reestablished, significant pump flow occurred and the pump suction leg was again emptied. This process of alternately depleting and refilling the pump suction leg was repeated several times during the ECC injection period, as indicated in Figure 8 by the alternate increase and decrease in measured density at the pump suction inlet from 20 to $64 \mathrm{sec}$ after blowdown initiation.

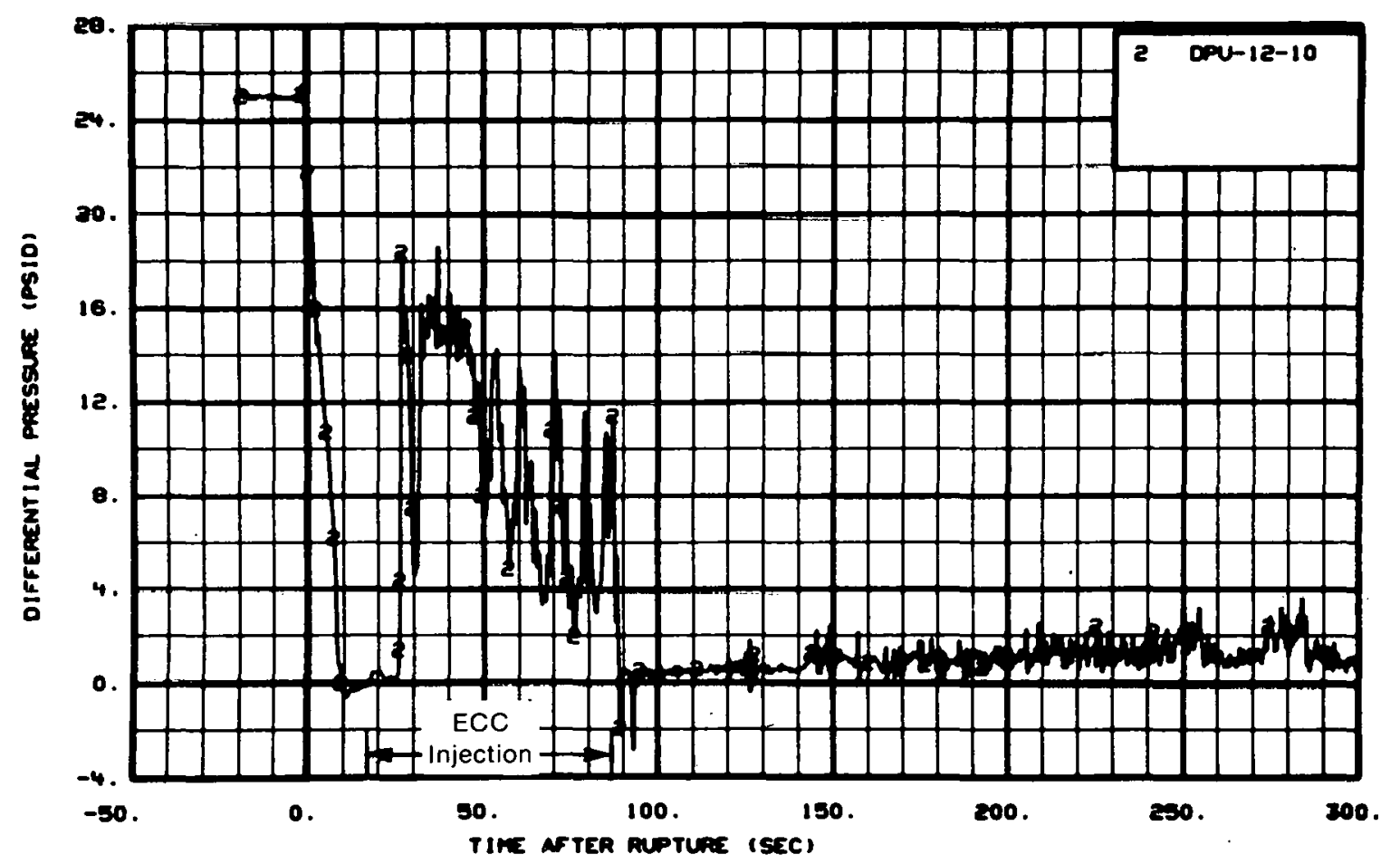

Fig. 2 Intact loop pump differential pressure (Test S-05-2) . 


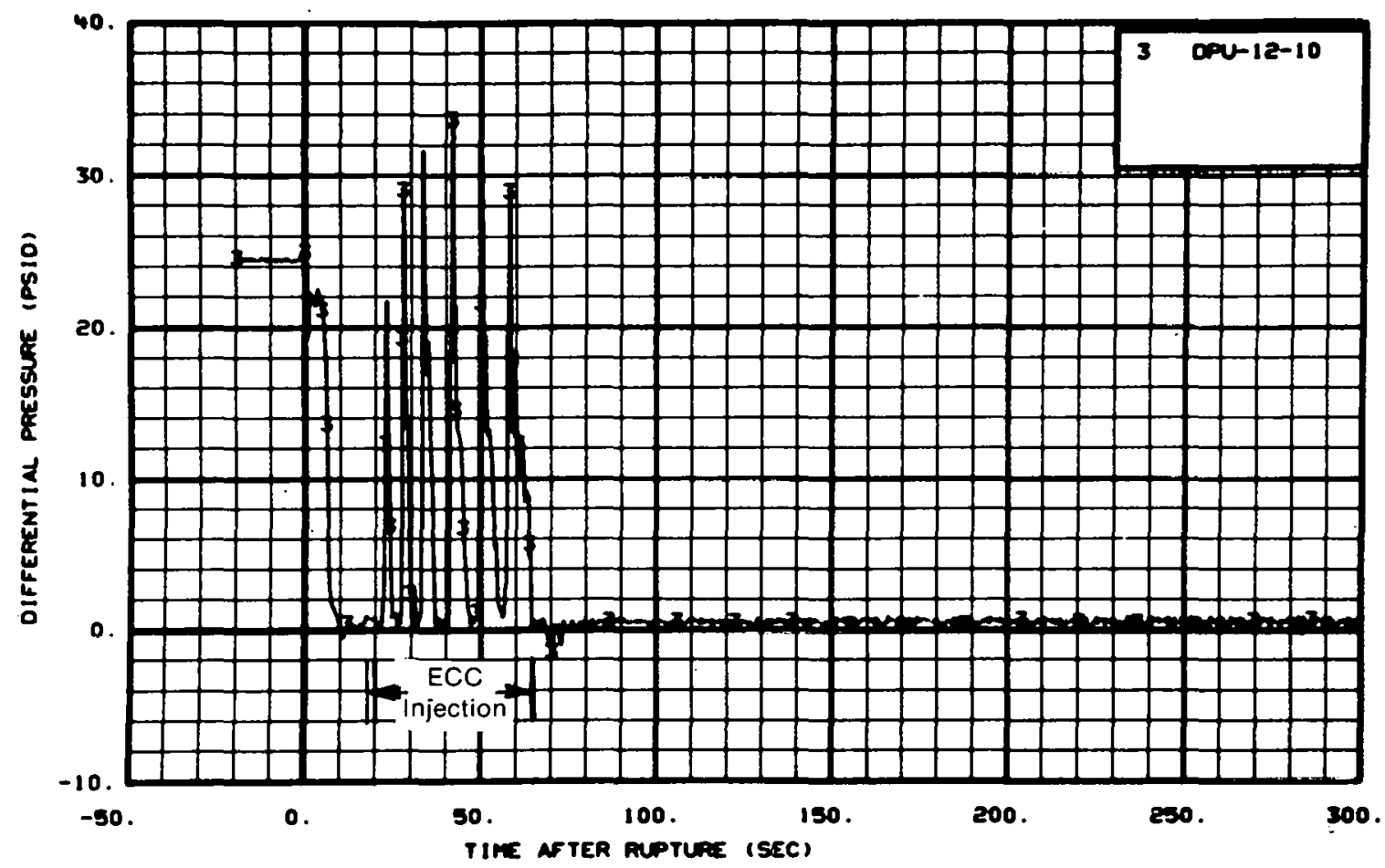

Fig. 3 Intact loop pump differential pressure (Test S-05-2A).

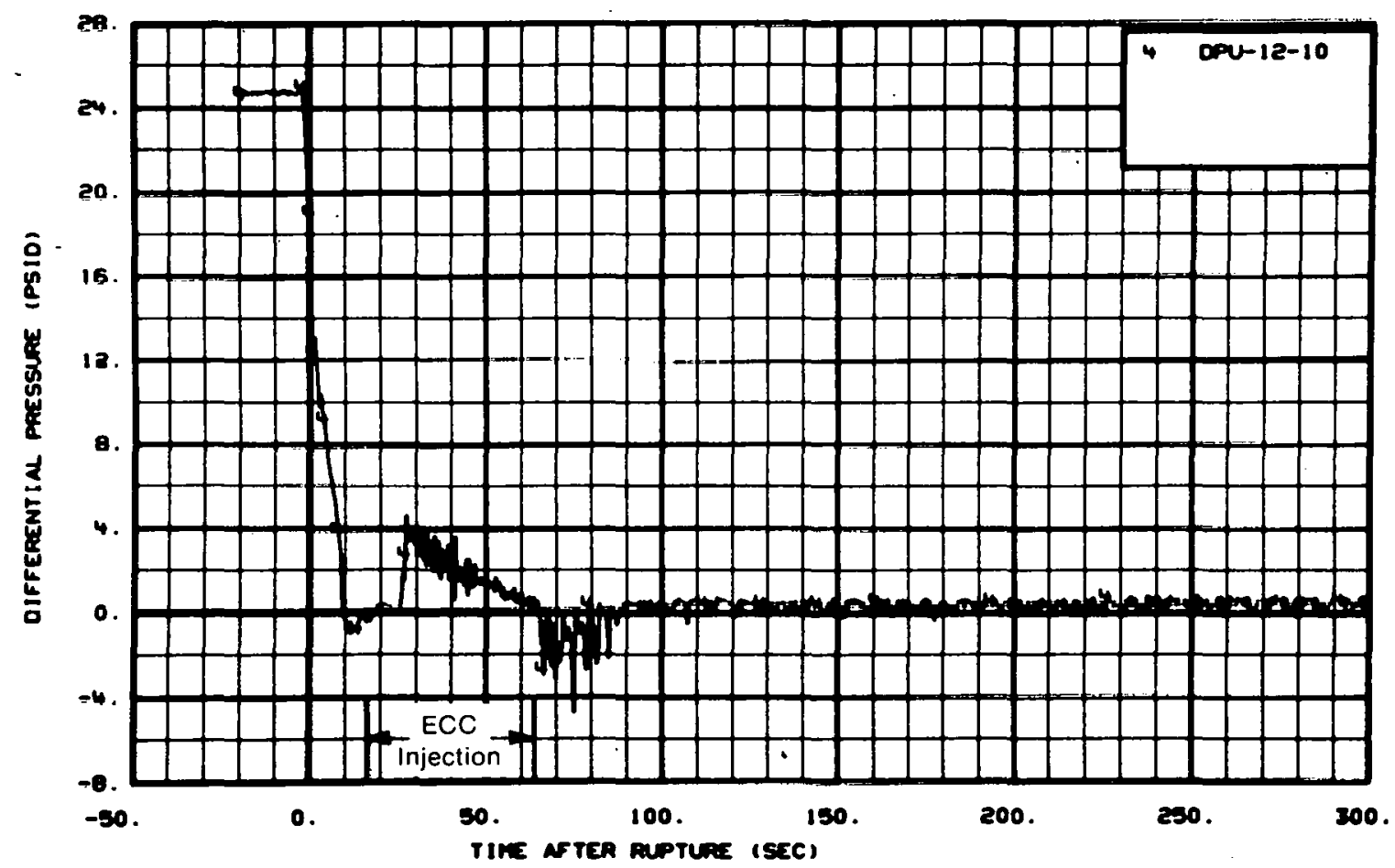

Fig. 4 Intact Innp pump differential pressure (Test S-05=2B). 


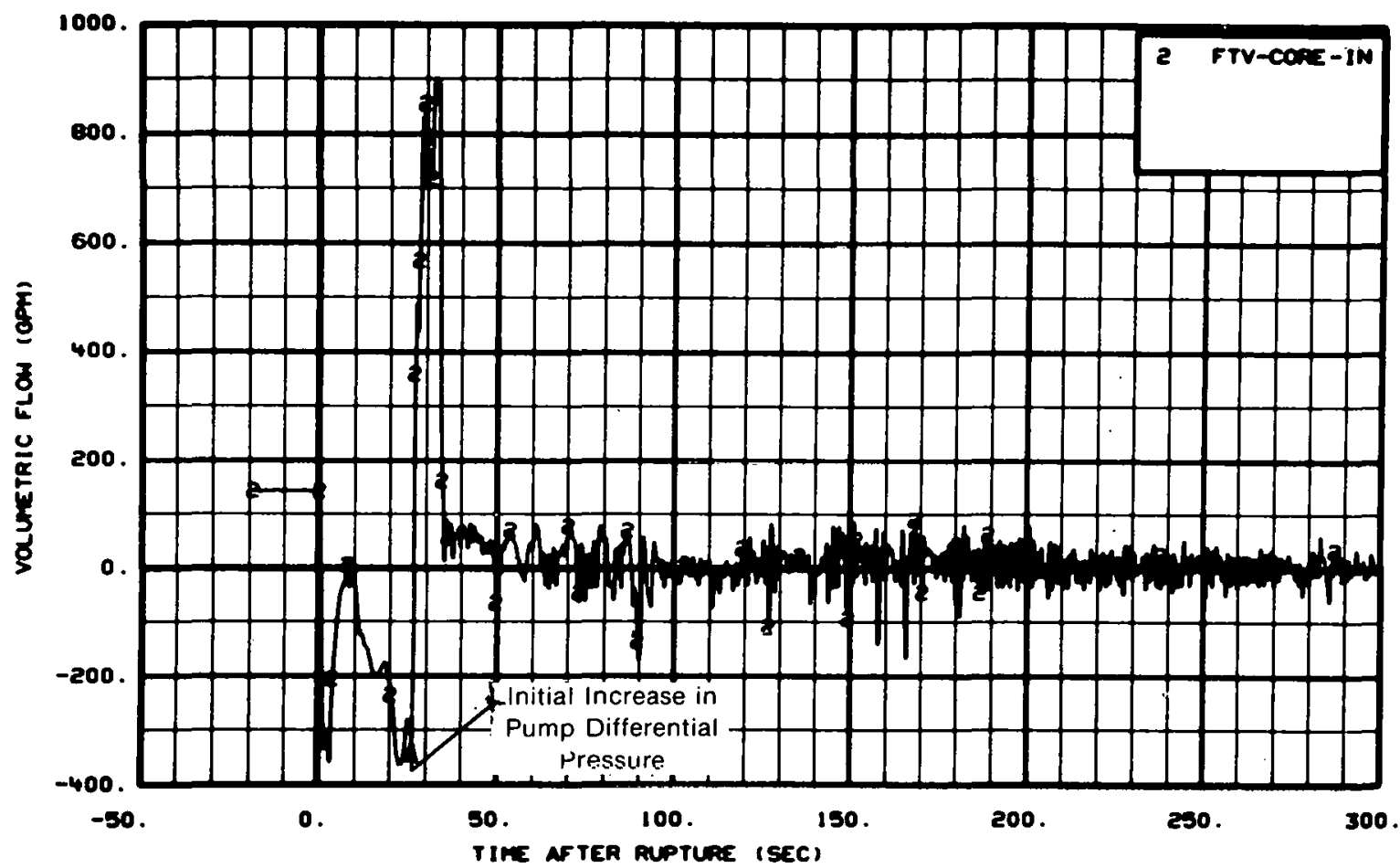

Fig. 5 Core inlet volumetric flow for Test S-0S-2.

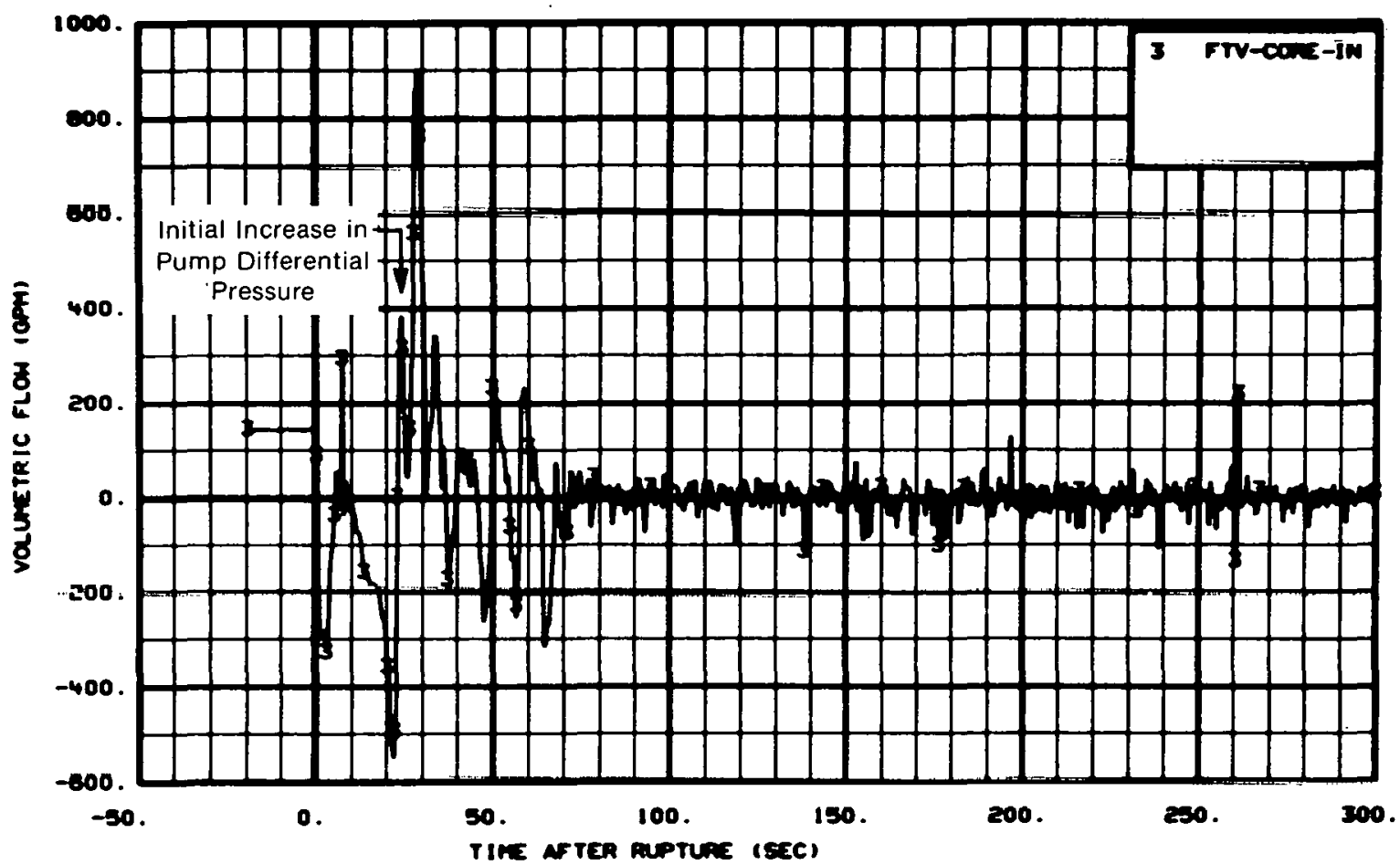

Fig. 6 Core inlet volumetric flow for Test S-05-2A. 


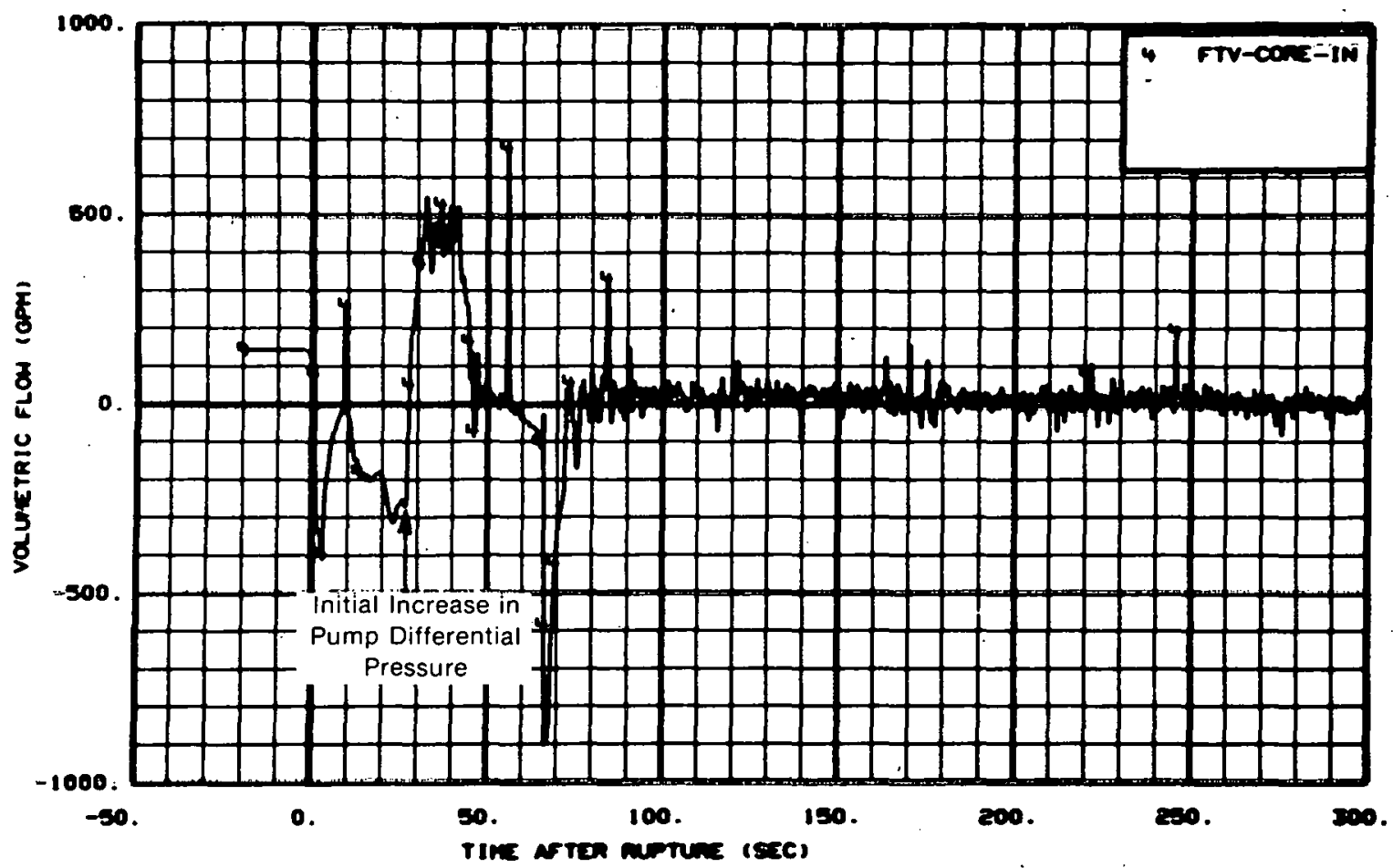

Fig. 7 Core inlet volumetric flow foŕ Test S-05-2B.

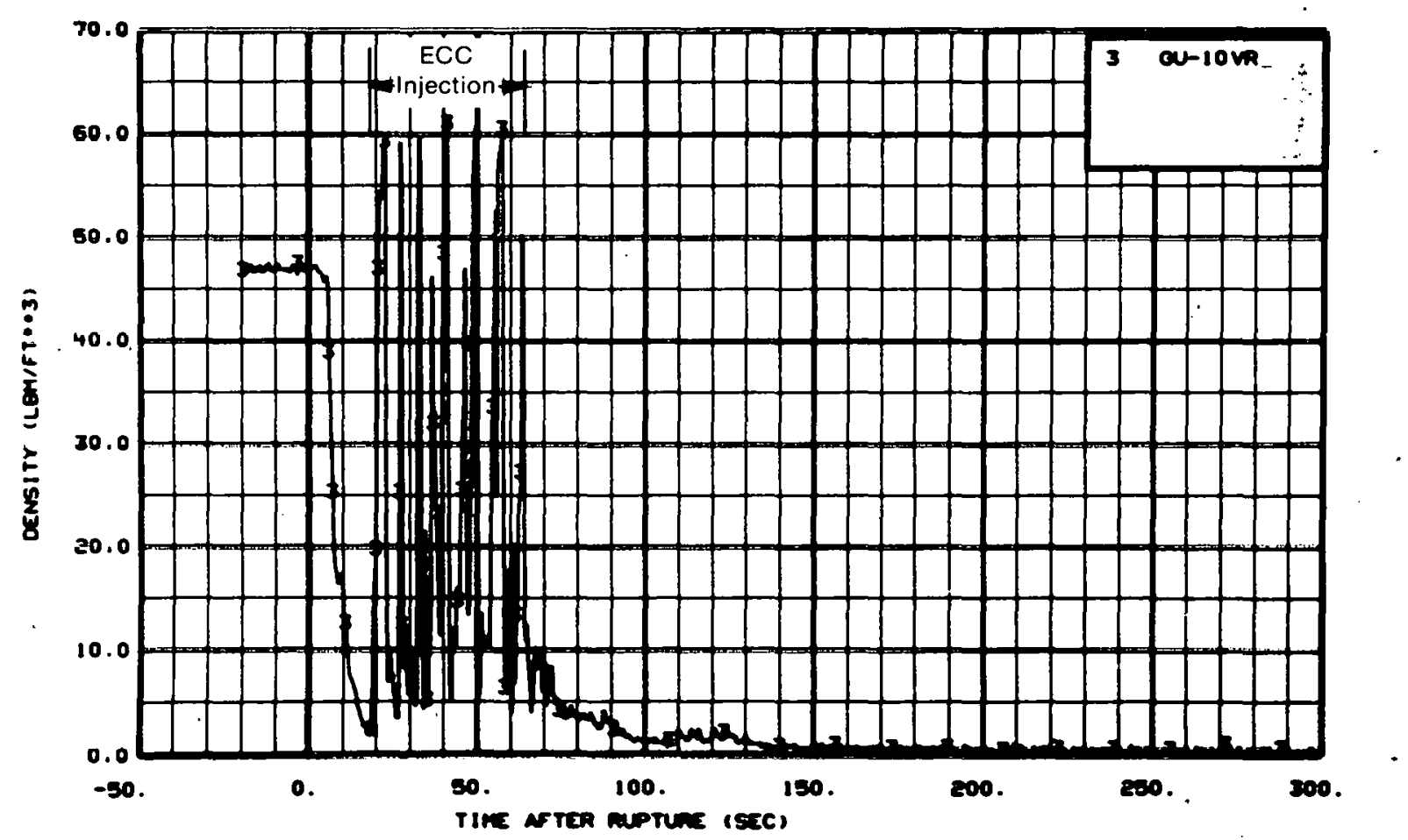

Fig. \& Density at pump suction inlet (Icst S-US-2A). 
The oscillations in the differential pressure across the pump apparently resulted in the corresponding oscillations in core inlet flow (Figure 6) which were observed in Test S-05-2A from 20 to $64 \mathrm{sec}$ after rupture. (Similar oscillations were also observed in the intact loop hot leg flow.)

Figure 2 shows that oscillations in the pump differential pressure also occurred in Test S-05-2 during the latter portion of the ECC injection period (46 to $89 \mathrm{sec}$ after rupture). In the first $22 \mathrm{sec}$ after the initial rise in differential pressure across the pump, the ECC injection rate in Test S-05-2 was apparently sufficient to keep the pump suction leg full of water while the pump operated at approximately $64 \%$ of prerupture speed. However, as the ECC injection rate decreased from about 28 to $14 \mathrm{gpm}$, the pump was able to empty the pump suction leg of water, and the pump pressure differential was observed to oscillate as the water in the pump suction leg was alternately depleted by the pump and then replaced by the ECC injection. The emptying and retilling of the puith $p$ suction leg was reflected in large oscillations in the pump suction leg density, which started to occur about $46 \mathrm{sec}$ after rupture.

Calculations based on approximate ECC injection rates indicate that the time required to fill the pump suction leg corresponds approximately to the frequency of the observed oscillations in Tests S-05-2 and S-05-2A. The pump speed in Test S-05-2B decayed to zero approximately $62 \mathrm{sec}$ after rupture. As a result, the pump speed was never sufficient to deplete the pump suction leg of water and, as indicated in Figure 4, the oscillations in pump differential pressure observed in Tests S-05-2 and S-05-2A never occurred in Test S-05-2B.

With pump suction injection, pump speed alone does not uniquely determine the intact loop hydraulic characteristics. A definite relation exists between the pump speed, which determines pump flow capacity, and the pump suction leg ECC injection rate. The relation of these two variables significantly affects overall pump performance which, in turn, influences thermal-hydraulic characteristics throughout the system.

Differences in pump performance characteristics affected the amount of intact loop ECC that was bypassed out the cold leg break in each of the pump suction injection tests. Comparison of broken loop cold leg density measurements during the ECC injection period, shown in Figures 9, 10, and 11, indicates more intact loop ECC was bypassed out the cold leg break in Tests S-05-2A and S-05-2B (as indicated by the higher average densities) than in T'est S-05-2.

Although differences in ECC injection rates may account for some of the differences in hypass amounts, the smaller amount of ECC water bypassed out the cold leg break in Test S-05-2 is thought to be due largely to the relatively constant pump differential pressure, which maintained a predominantly positive core flow during intact loop ECC injection (Figure 5). Although positive spikes in the pump differential pressure rise in Test S-05-2A (Figure 3) indicate oscillations in the pump differential pressure as high as 34 psi (compared with a maximum pump differential pressure of 18 psi in Test S-05-2), alternate drops in the developed pump differential pressure caused by the depletion of ECC in the pump suction leg apparently allowed intermittent core flow reversals to occur 


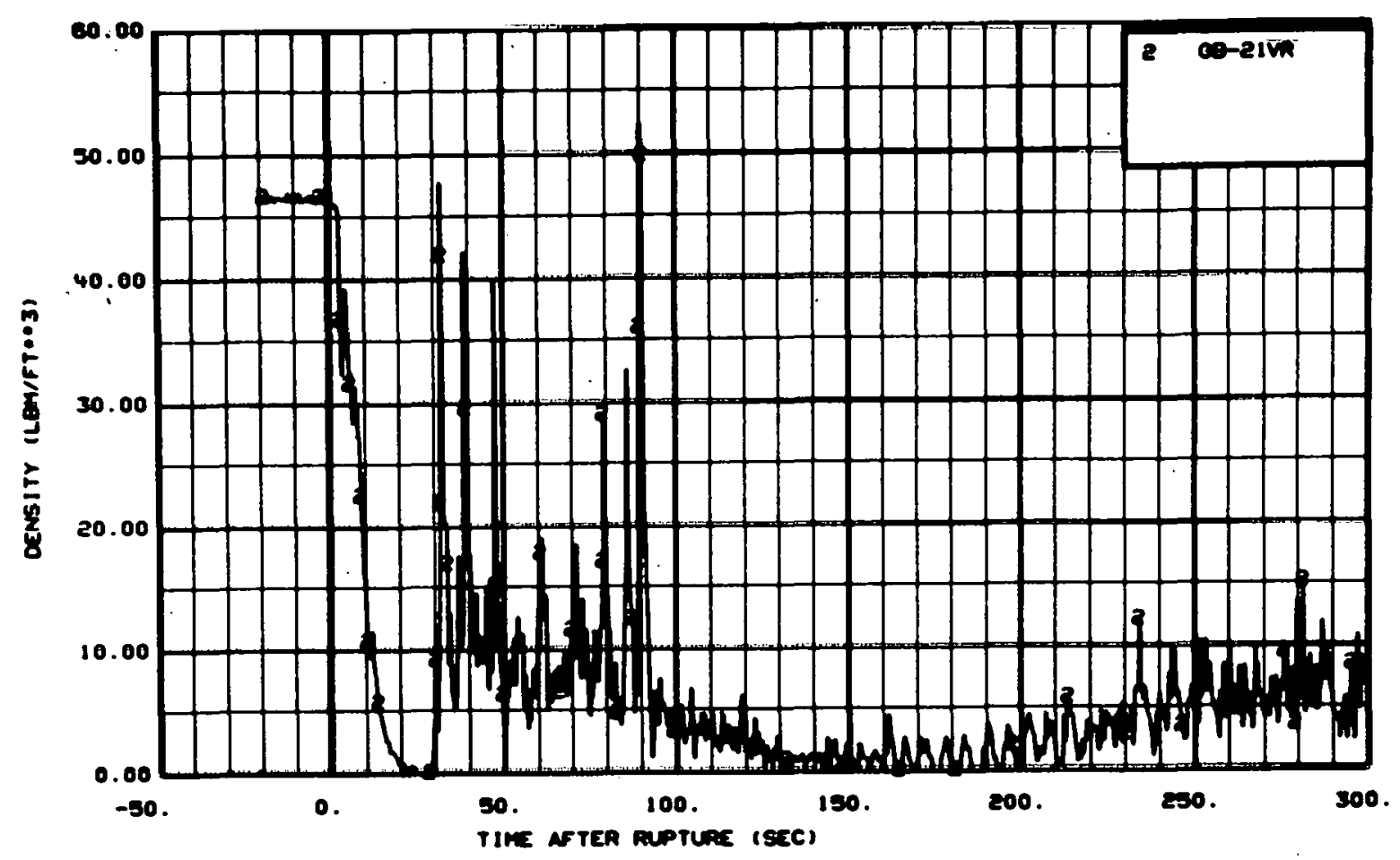

Fig. 9 Density at broken loop cold leg inlet nozzle (Test S-05-2).

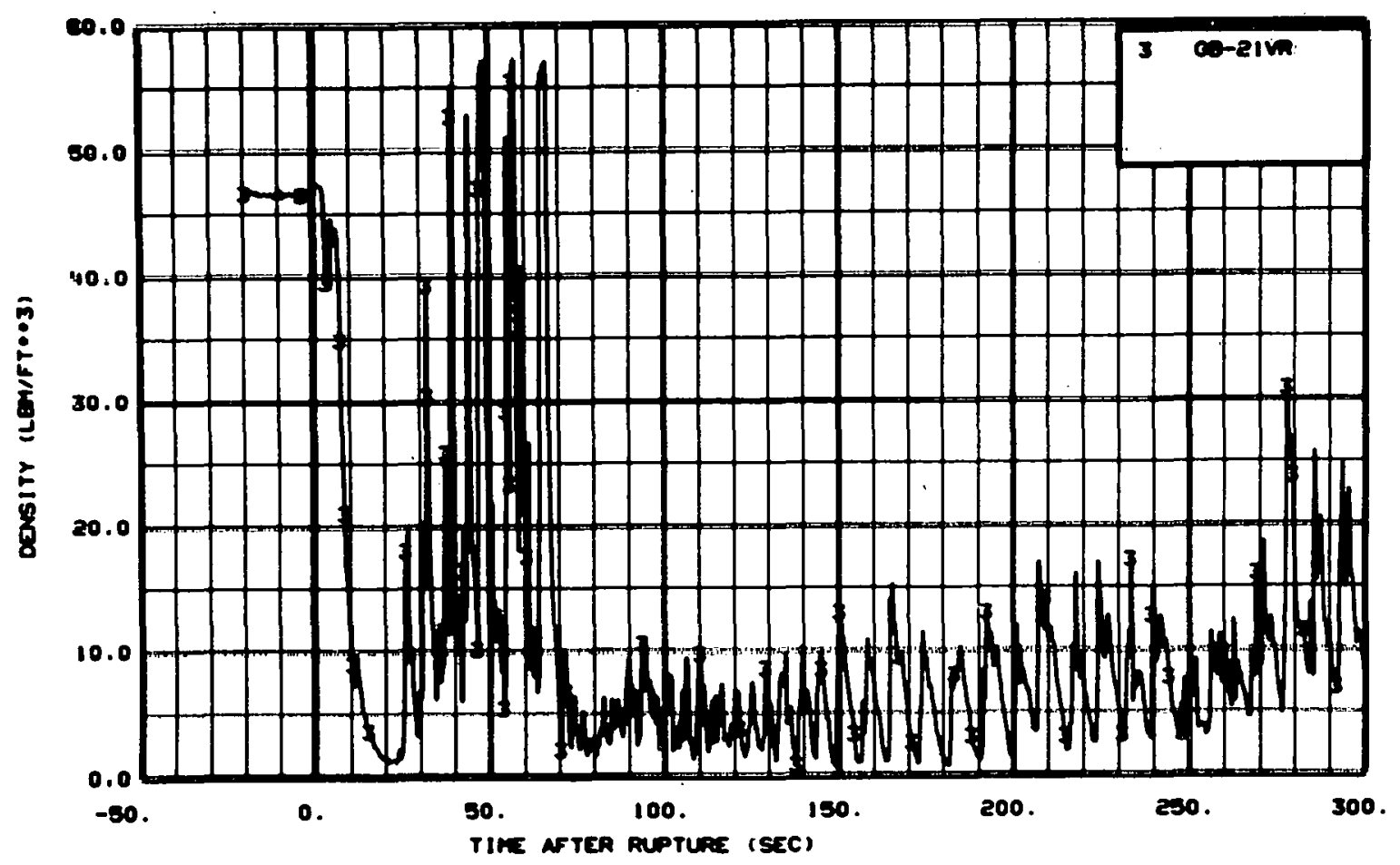

Fig. 10 Density at. hroken lonp cold leg inlet nozzle (Test S-05-2A). . 


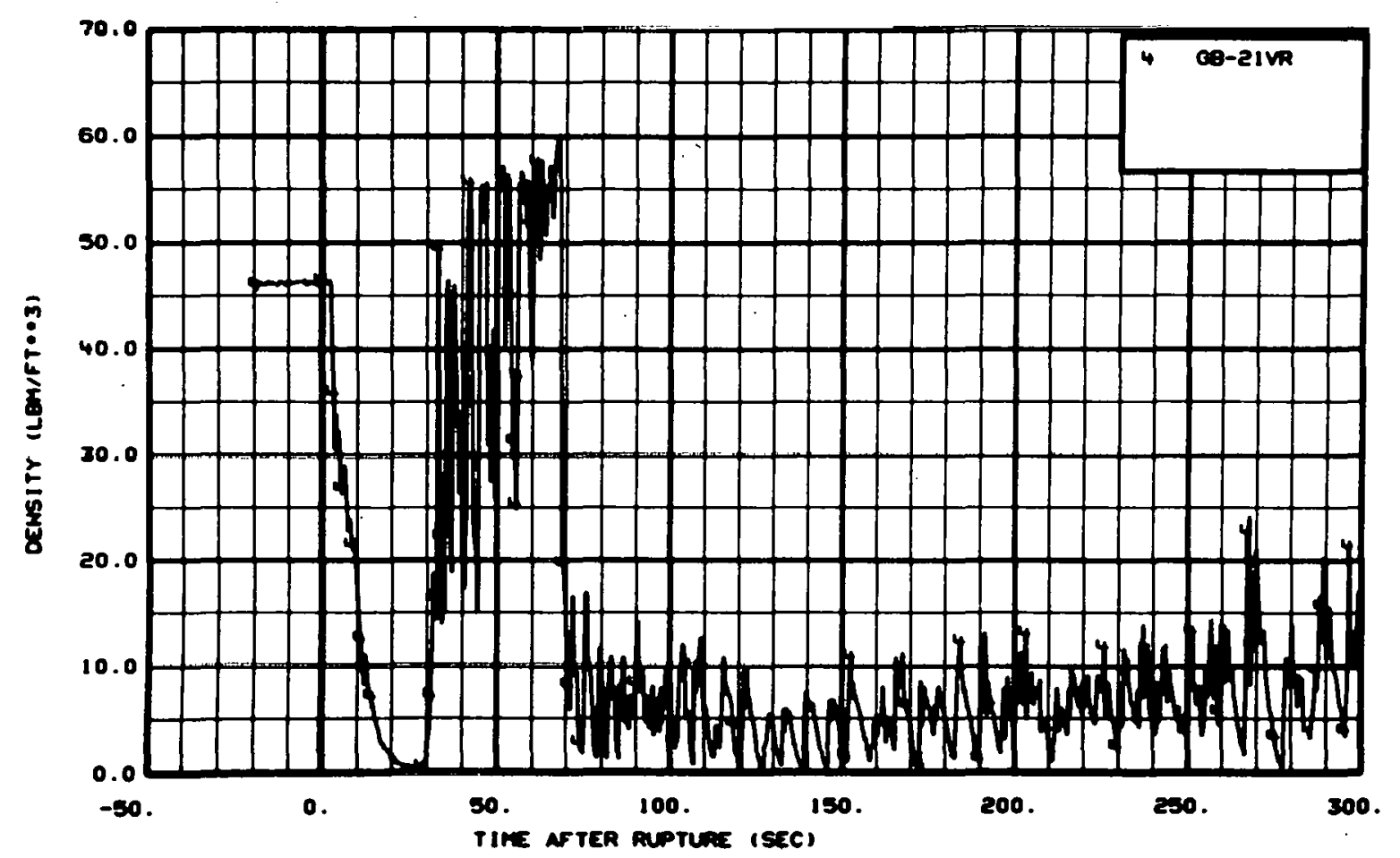

Fig. 11 Density at broken loop cold leg inlet nozzle (Test S-05-2B).

(Figure 6). These reverse core flows, and the resultant flows up the downcomer, apparently carried substantial intact loop ECC out the cold leg break, as indicated by the peaks in the broken loop cold leg density measurements in Figure 10, which approximately correspond to the periods of reverse flow out the bottom of the core. In Test S-05-2B, the relatively large bypass out the cold leg break (as indicated by the increased density in Figure 11) appears to be due, in part, to a lack of substantial pump head to force ECC down the downcomer and into the lower plenum. The core inlet density observed in Test S-05-2B indicated very little water reached the bottom of the core during the ECC injection period (18 to 64 sec after rupture).

The general trend in the downcomer liquid inventory during ECC injection at the pump suction was similar in the three tests, although more liquid accumulated in the downcomer during ECC injection in Test S-05-2. Figure 12 shows the collapsed liquid level in the downcomer for each of the tests. The collapsed liquid levels were calculated from the pressure drop between pressure taps, which were 166 in. and 9 in. below the cold leg centerline. As shown in Figure 12, during intact loop accumulator injection, little mass accumulated in the downcomer in any of the tests. Although the broken loop flow and density measurements in Tests S-05-2A and S-05-2B indicate substantial ECC bypassed the downcomer, apparently such was not the case in Test S-05-2. Flow and density measurements at the core inlet for Test S-05-2 indicate that a significant amount of ECC water entered the core. Therefore, the pump must have effectively forced the fluid down the downcomer and into the core before a liquid head in the downcomer was established in Test S-05-2. The increased flow of liquid up the core in Test S-05-2 was also indicated by 


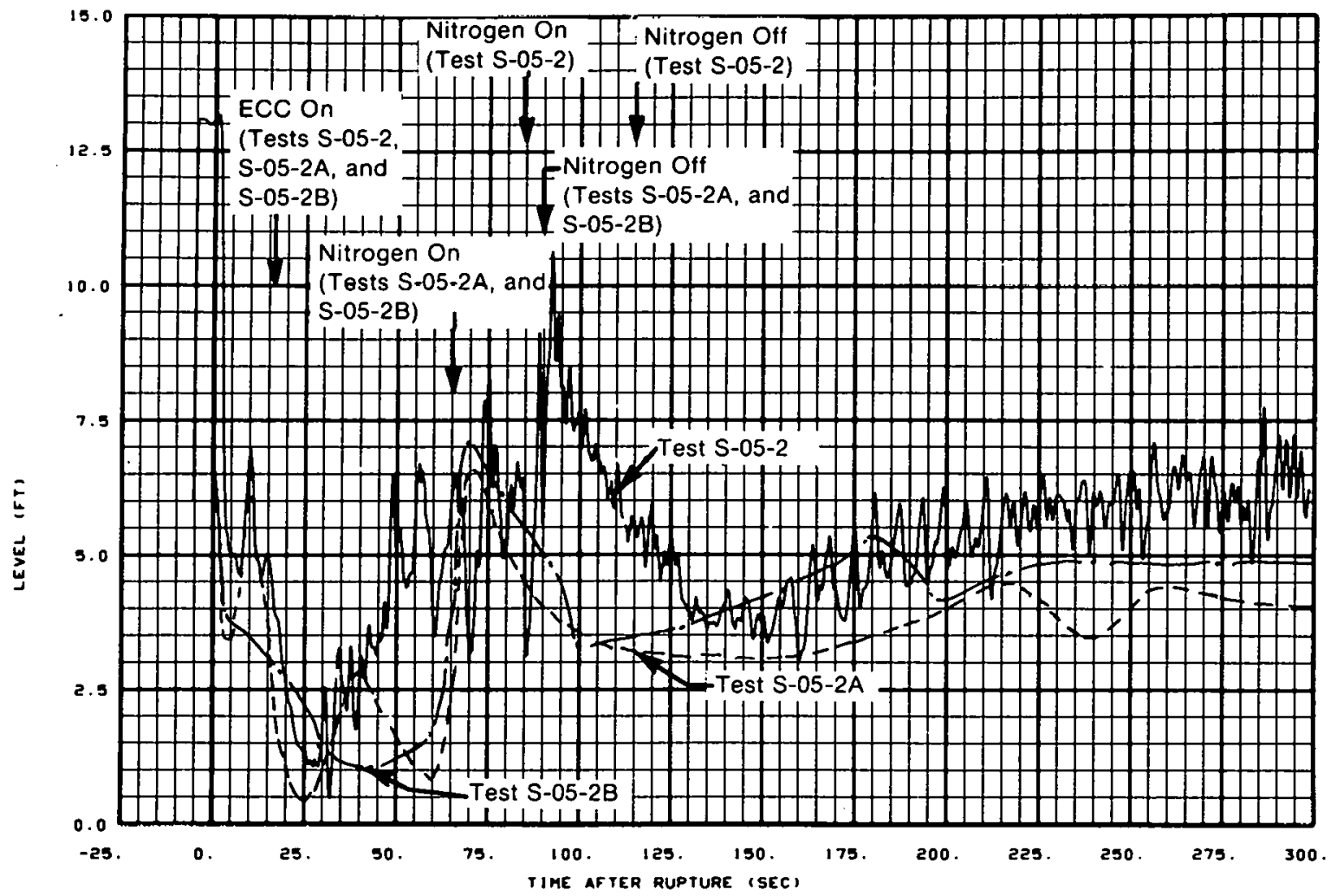

Fig. 12 Downcomer collapsed liquid level.

increased densities at the inlet to both the broken loop and intact loop hot legs, indicating significant liquid flow out the hot legs from 45 to $95 \mathrm{sec}$ after rupture. 'The presence of the liquid apparently resulted from entrainment in the core followed by carryover.

The differences in pump behavior which led to differences in core flow characteristics also resulted in significantly different core temperature response characteristics between Tests S-05-2, S-05-2A, and S-05-2B. However, not all the observed differences in core temperature response were directly attributed to the pump behavior. The core temperature response at the 13-in. elevation is shown in Figure 13. Comparison of the temperature response for Tests S-05-2 and S-05-2A from 9 to $60 \mathrm{sec}$ after rupture indicates that the earlier quench time for Test S-05-2 could be partly due to the higher peak temperature that occurred during blowdown in Test S-05-2A. Since the slope of the temperature response curves shown in Figure 13 are very nearly the same for Tests S-05-2 and S-05-2A from about 9 to $50 \mathrm{sec}$ after rupture, the core heat transfer at the 13-in. elevation for this time period should be essentially the same for the two tests. Similar core cooling characteristics for Tests S-05-2 and S-05-2A from 9 to $50 \mathrm{sec}$ after rupture were observed in most of the lower core temperature response measurements up to the 21 -in. elevation. Above the 21 -in. elevation, the core heat transfer during the period of maximum developed pump differential pressure was clearly less in Test S-05-2A than in Test S-05-2, as is evidenced in Figure 14 by the decreased slope of the temperature curve ( 9 to $50 \mathrm{sec}$ after rupture) of Test S-05-2A at the 29-in. elevation (rod hot spot) compared with that of Test S-05-2. Therefore, although core heat transfer characteristics in Tests S-05-2 and S-05-2A were similar in the lower portions 


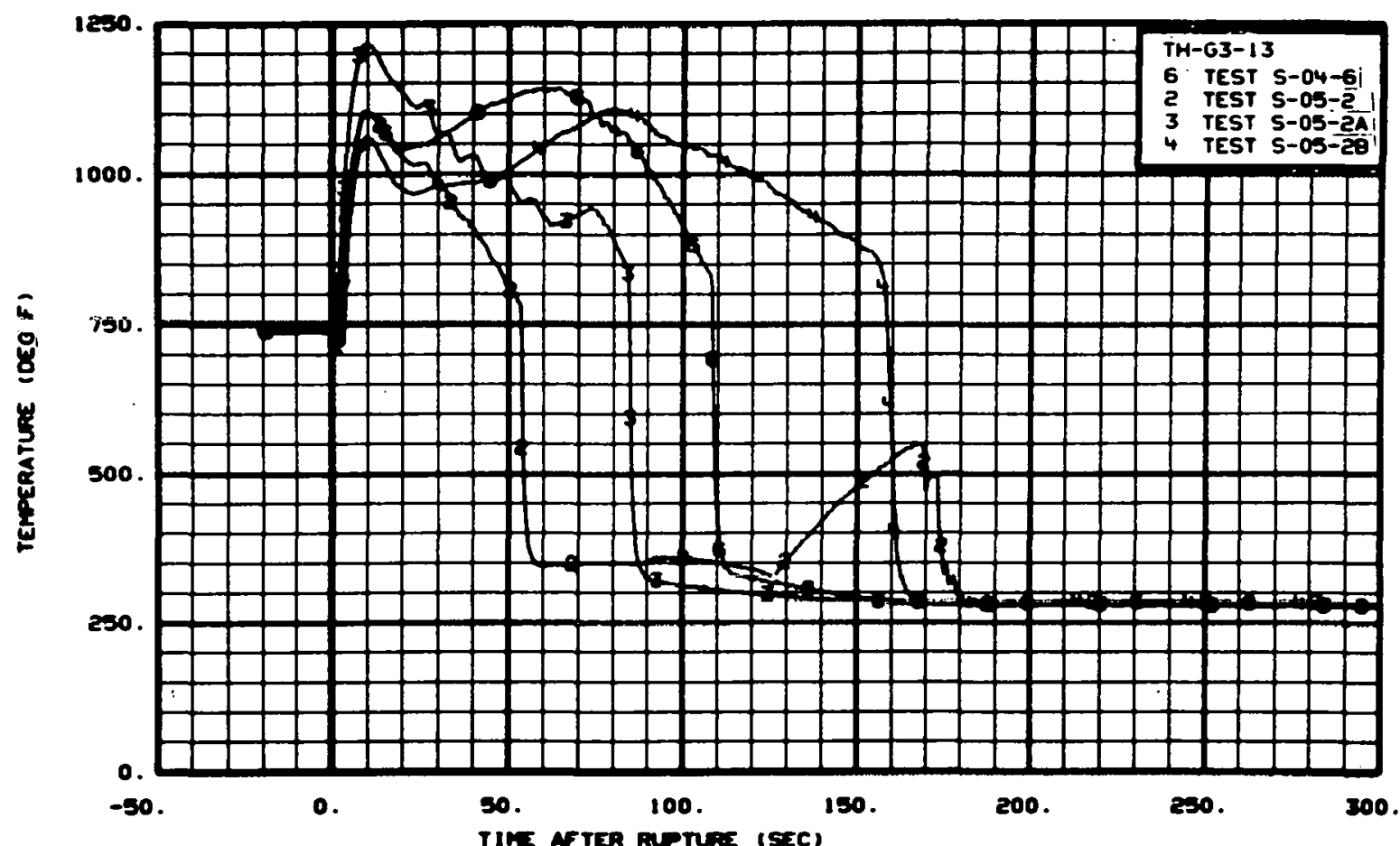

Fig. 13 Rod temperature at the 13-in. elevation.

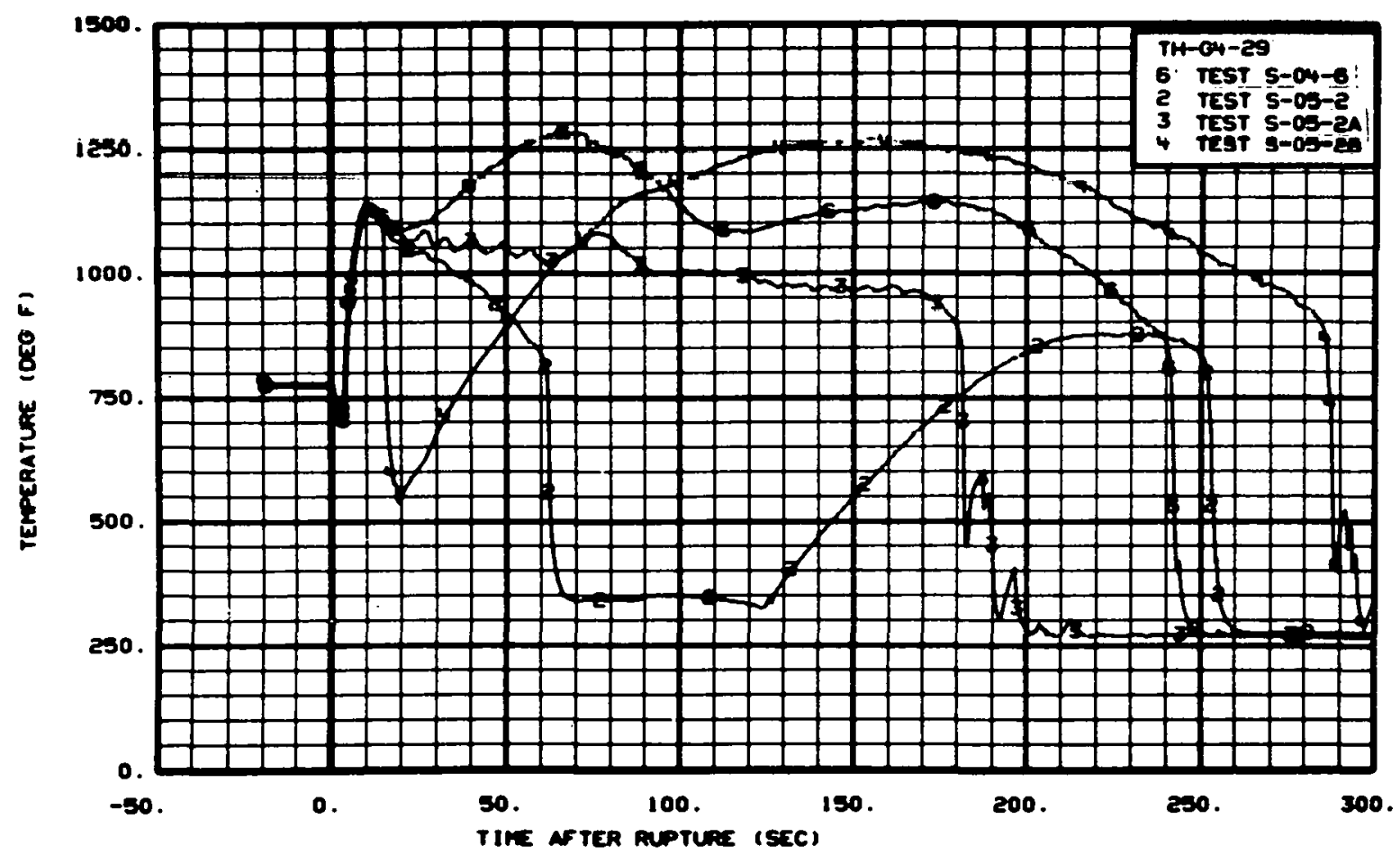

Fig. 14 Rod temperature at the 29-in. elevation. 
of the-core, differences in pump behavior and core flow apparently resulted in better cololing of the upper portions of the core for Test S-05-2. The oscillations in the core temperature response curves for Test S-05-2A in Figure 13 and 14 at the 13- and 29-in. core elevations, respectively, are thought to be caused by the pump behavior, because the frequency of the temperature oscillations corresponds to the frequency of the pump differential pressure and core inlet flow oscillations.

Despite an initial drop in the temperature response at the 29-in. elevation for Test S-05-2B, as evidenced by Figure 14, indicating substantial initial core cooling between 9 and $18 \mathrm{sec}$ after rupture, the second rise in heater rod temperature beginning $18 \mathrm{sec}$ after rupture indicates a significantly lower core heat transfer during pump suction ECC injection in Test S-05-2B than in either Test S-05-2 or Test S-05-2A. The peak of this second rise in rod cladding temperature during Test S-05-2B in many cases exceeded the peak reflood temperature observed in Test S-04-6. Figure 15 is typical of several temperature measurements above the 29-in. elevation and shows the peak cladding temperature during reflood in Test S-05-2B exceeding the peak temperature observed in the baseline ECC injection test, Test S-04-6.

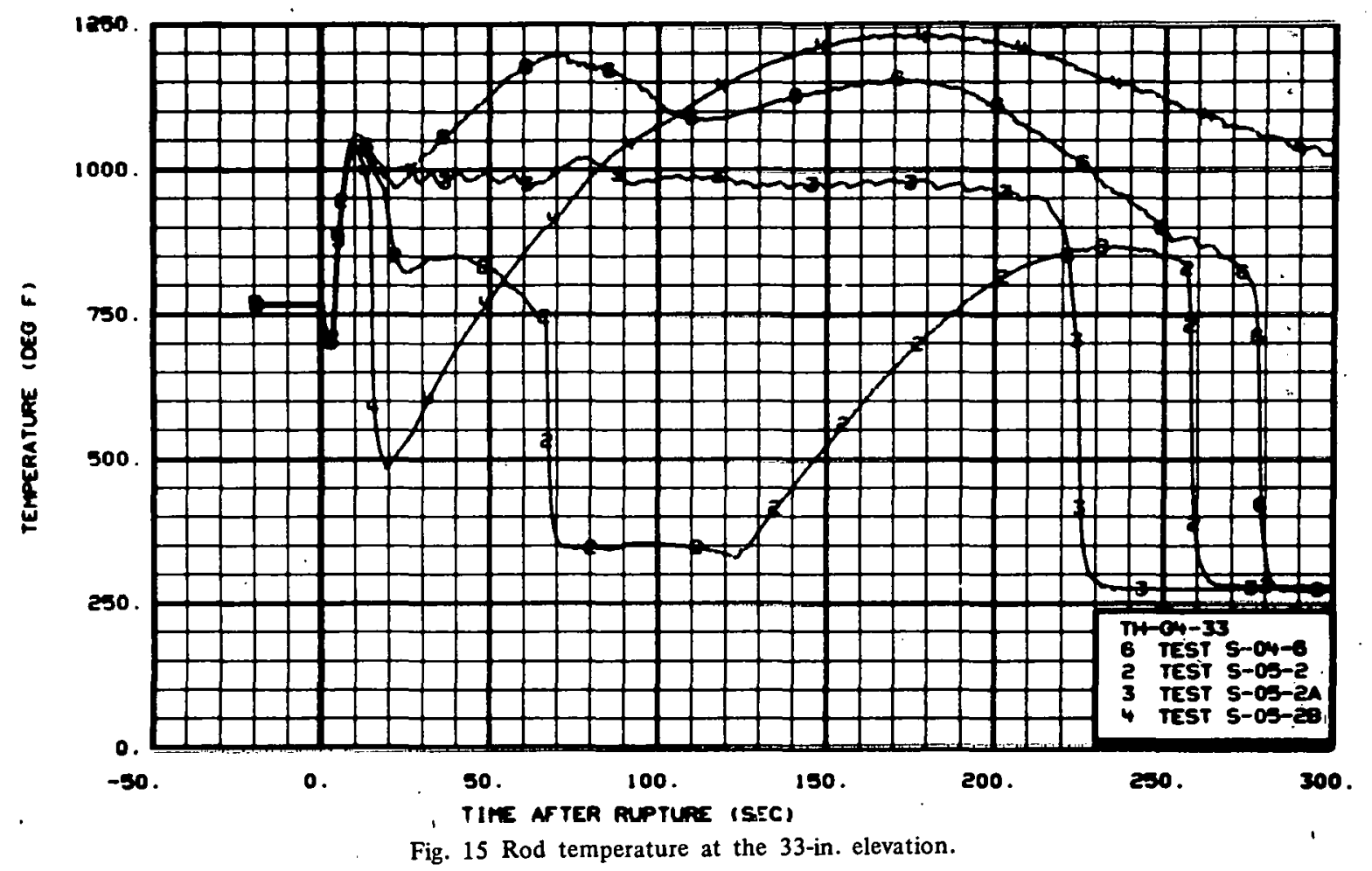

The late dryout of rods experienced in Test S-05-2 about $125 \mathrm{sec}$ after rupture (Figures 13, 14, and 15) was not observed in Tests S-05-2A and S-05-2B. The absence of dryout in Tests S-05-2A and S-05-2B appears to have been the result of the relocation of the low pressure injection system injection point from the pump suction leg in Test S-05-2 to the intact loup cold leg in Tests S-05-2A and S-05-2B. 
Overall, better cooling of the core occurred in Test S-05-2 than in either Test S-05-2A or Test S-05-2B. Test S-05-2A apparently provided the next best core cooling, despite significant oscillations in the pump differential pressure, and appeared to provide better cooling of the core than was observed in the baseline test (Test S-04-6). Significantly less core cooling was observed in Test S-05-2B than in either of the other two pump suction injection tests or the baseline test. In fact, very little of the core above the 29 -in. elevation in Test S-05-2B appeared to have quenched $300 \mathrm{sec}$ after rupture.

On the basis of the previous discussion, during the early portion of the simulated loss-of-coolant accident (LOCA) when ECC was injected into the pump suction leg, the pump behavior significantly affected system response and core thermal behavior. The effect of the pump was also evident later in the transient during nitrogen injection. After intact loop accumulator water was exhausted, nitrogen flow from the accumulator in all three tests cleared the intact loop and the broken loop cold legs of water and increased the liquid inventory in the downcomer. Figure 12 showed that the collapsed liquid level in all three tests increased as nitrogen began to flow into the intact loop. At the start of nitrogen injection, the developed pump differential pressure degraded in Tests S-05-2 and S-05-2A. In addition, substantial reverse flow out the intact loop hot leg occurred in Tests S-05-2A and S-05-2B. A larger reverse flow spike was observed in Test S-05-2B than in Test S-05-2A. This larger flow spike is thought to be caused by the larger resistance to positive intact loop flow offered by the stationary pump in Test S-05-2B. As a result, more nitrogen flow injected into the pump suction leg was forced out the hot leg in the reverse flow direction. In Test S-05-2, the relatively small intact loop hot leg reverse flow spike observed when nitrogen began to flow from the intact loop accumulator (approximately $86 \mathrm{sec}$ after rupture) is thought to be due in part to a lower rate of nitrogen injection. The maximum rate of nitrogen injection in Test S-05-2 was about $35 \mathrm{gpm}$ as compared with $75 \mathrm{gpm}$ for Tests S-05 $\cdot 2 \mathrm{~A}$ and $\mathrm{S} \cdot 05 \cdot 2 \mathrm{~B}$.

In Tests S-05-2A and S-05-2B, the intact loop flow reversals that occurred when nitrogen began to flow from the intact loop accumulator (about $65 \mathrm{sec}$ after rupture) were accompanied by reversals in the core flows (Figures 6 and 7). The core flow reversal in Test S-05-2A resulted in a reduction in the core heat transfer as indicated by the localized peak in the core temperature response curves in Figures 13, 14, 15 (75 sec after rupture). A reduction in core heat transfer when nitrogen began to flow into the intact loop was not as apparent in either Test S-05-2 or Test S-05-2B. Further analysis of the effects of nitrogen injection on system flow and core thermal response characteristics with pump suction injection is required.

In conclusion, the results of Test S-05-2 show that ECC injection into the pump suction leg can result in good core cooling during a LOCA. However, excessive pump speed and a correspondingly high pumping capacity relative to the rate of ECC injection (as in Test S-05-2A) results in significantly degraded system thermal-hydraulic performance characteristics. Likewise, loss of pump power at the time of rupture, as simulated in Test S-05-2B, reduces the effectiveness of the ECC injection at the pump suction and may preclude effective cooling of the core. 


\section{EVALUATION OF CHF CORRELATIONS FOR USE IN CALCULATING}

\section{TIME OF CHF IN SEMISCALE}

\section{M. Snider}

Analysis was performed to evaluate the capability of common CHF correlations to calculate the time of the first CHF in the Semiscale core during a LOCA produced by a double-ended cold leg pipe rupture. In the large cold leg break tests with the system initially at 2250 psia and full power (1.6 MW), the core inlet flow reversed direction within $50 \mathrm{msec}$, and a CHF generally occurred in parts of the core within $800 \mathrm{msec}$ after rupture, and in some cases as early as $\mathbf{4 8 0} \mathrm{msec}$ after rupture.

Most CHF correlations have been developed from experiment data obtained at near steady state conditions. The phenomenon of a boiling crisis is transient, and correlations generated from quasi-steady test conditions may also apply to transients such as those which occur during a cold leg break LOCA (a depressurization transient and a simultaneous flow transient). By evaluating the capability of common CHF correlations to calculate a boiling crisis in the Semiscale core, support for the hypothesis that existing CHF correlations are applicable to LOCA analysis can be gained. The capability of the correlations was evaluated by comparing the rod surface heat flux at the time of a boiling crisis with the CHF calculated from the correlations. The hydraulic conditions used in the CHF correlations for these calculations were derived from COBRA-IV-I[5] FIX3[a] calculations obtained using boundary conditions from measured data. Results from the hydraulic analysis are discussed in Reference 6.

In this section; the following seven CHF correlations were evaluated: MacBeth ${ }^{[7]}$; Biasi $^{[8]}$; Barnett ${ }^{[9]}$; Babcock \& Wilcox Company, B\&W-2 ${ }^{[10]}$; Westinghouse Electric Corporation, W-3 $[11] ;$ LOFT; and General Electric Company Corporation (Hench-Levy) ${ }^{[12]}$.

The Loss-of-Fluid Test (LOFT) correlation, which is unpublished, is of the following form:

$$
{ }^{q_{C H F}}=0.12947 G+710 p-0.23018 p^{2}-2.9563 G x+0.001004 G p x
$$

where

$$
\begin{aligned}
\mathrm{G} & =\text { mass flux in } 1 \mathrm{~b}_{\mathrm{m}} / \mathrm{hr}_{\mathrm{ft}}{ }^{2} \\
\mathrm{p} & =\text { pressure in psia (applicable pressure range } 2000 \text { to } 2200 \text { psia) } \\
x & =\text { quality. }
\end{aligned}
$$

[a] Code configuration control H00062IB. 
Correlations which contained the distance from the core inlet to the point of the CHF and an inlet enthalpy as independent variables (Westinghouse Electric Corporation W-3 and the Barnett correlations) were not applicable to transient analysis and therefore were modified. The modification was made using a constant heat flux of the same value as the local heat flux from the inlet to the location of CHF. Through use of steady state conservation of energy, the equations were transformed to local condition correlations.

Figure 16 compares the calculated CHF from correlations with the experiment mean heat flux. The mean heat flux was calculated from 22 fluxes obtained from temperature data within the peak power zone $(21 \text { to } 31 \mathrm{in.})^{[\mathrm{a}]}$. A boiling crisis was measured to occur

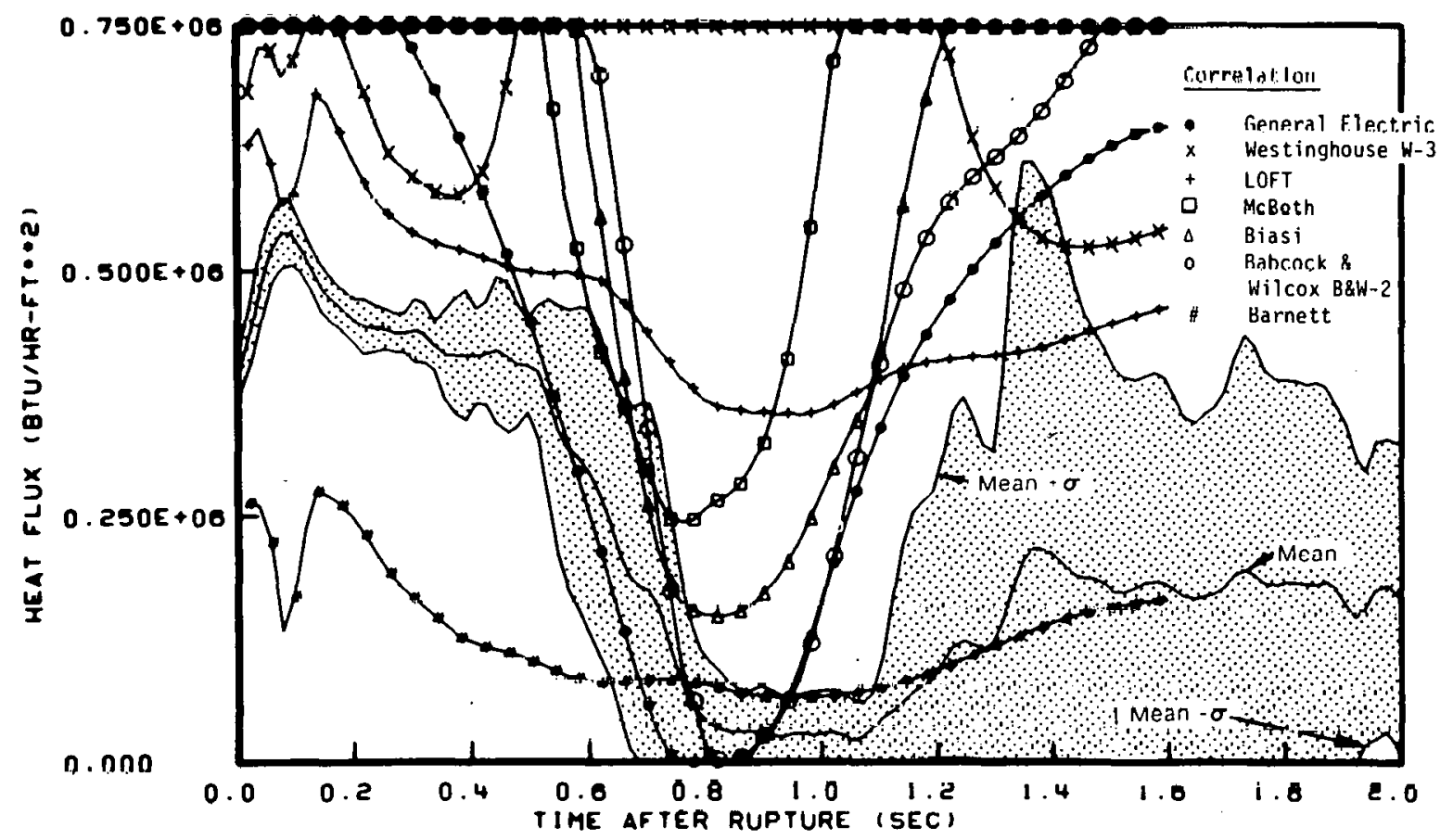

Fig. 16 Calculated critical heat fluxes compared with the mean heat flux at the peak power step (21 to 31 . in).

when the surface heat flux rapidly decreased. A boiling crisis was calculated to exist only if the calculated CHF was below the measured heat flux. The time at which the calculated $\mathrm{CHF}$ and measured heat flux curves intersect was the calculated CHF time.

The heat flux calculated by the General Elcctric Company (GE) correlation is shown in Figure 16 to intersect the mean heat flux as the mean heat flux begins to decrease, therefore providing an excellent calculation of the time to CHF. The good agreement between the GE correlation and the data is as would be expected from the algebraic form of the equation and from the large and rapid increase in quality, shown in Figure 17, during the period of CHF ( 500 to $600 \mathrm{msec}$ ). The GE-CHF equation is a simple linear function of

[a] Distances are referenced to the bottom of the heated core. 


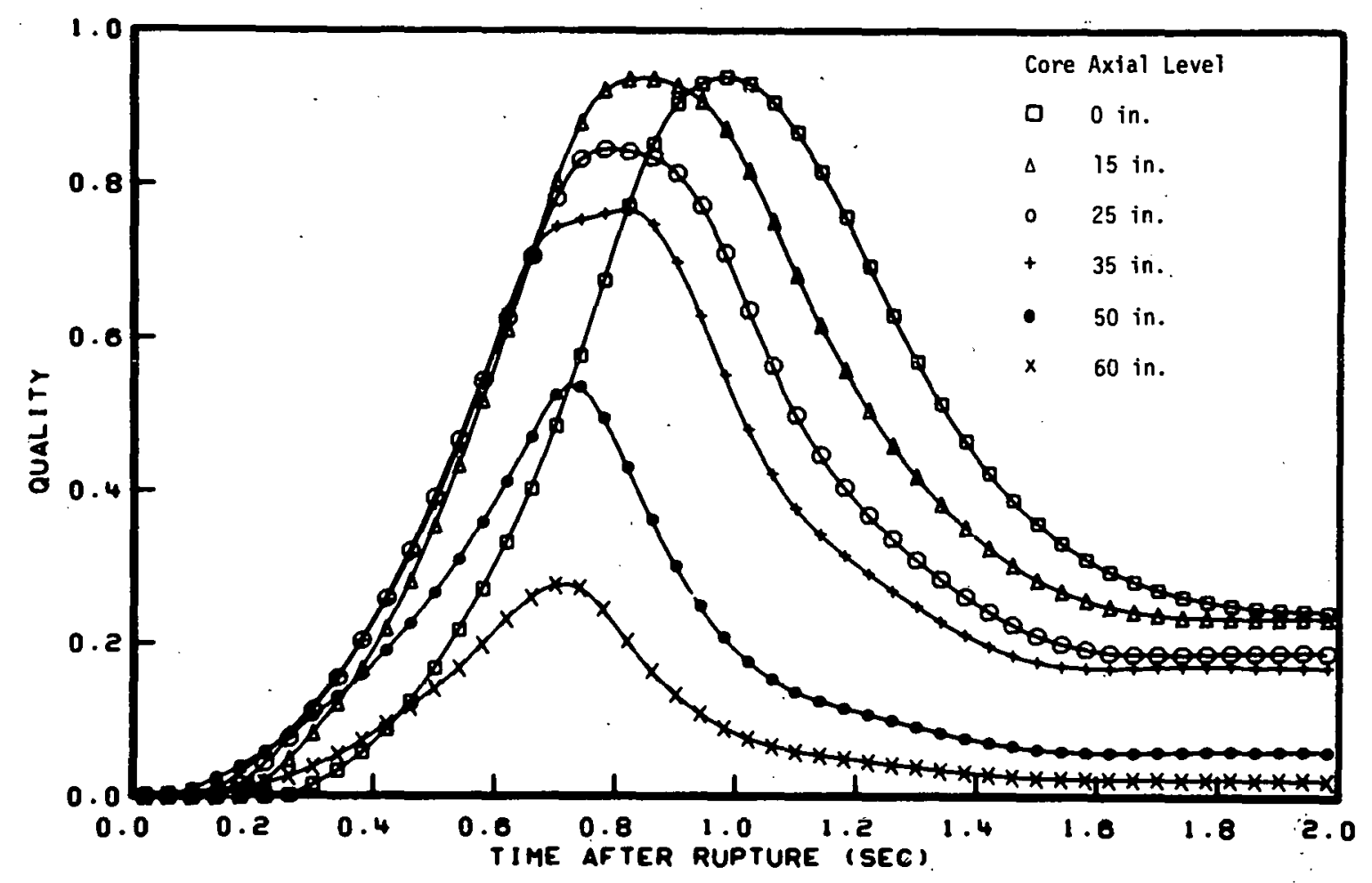

Fig. 17 COBRA-IV-I calculated quality at axial core levels.

quality ${ }^{[12]}$, and during the period of a large rise in quality, the calculated CHF decreased below the mean heat flux. As shown in Figure 16, the MacBeth, B\&W-2, and Biasi correlations, which also strongly depend on quality, calculate close to the same time for $\mathrm{CHF}$, and this time was $150 \mathrm{msec}$ later than the mean $\mathrm{CHF}(500 \mathrm{msec})$. The Westinghouse W-3 correlation modified for transient conditions did not calculate that a critical heat flux would occur. The calculated $\mathrm{CHF}$ from the W-3 correlation reached a minimum value at about $350 \mathrm{msec}$ and then increased to values outside the range of the figure. The large calculated CHF after $350 \mathrm{msec}$ was the result of an exponential term in the equation rapidly increasing as quality increased above $20 \%$. The LOFT correlation also did not calculate that a CHF would occur, and the Barnett correlation modified for transient conditions calculated a CHF well below the measured heat flux even prior to rupture, indicating $\mathrm{CHF}$ would occur at steady state conditions.

The results from the correlations are compared with the mean heat flux in the lower and upper parts of the core in Figures 18 and 19, respectively. The lower part of the core is from 11 to 16 in., and the mean flux value in this region was calculated from seven fluxes. The upper part of the core is from 31 to $36 \mathrm{in}$., and the mean heat flux was calculated from eight fluxes. In the lower part of the core, the GE correlation again calculates the CHF well. The mean CHF occurred at about the same time as that for the peak power step $(560 \mathrm{msec})$, and the quality, as seen in Figure 17, rose at about the same rate as that in the peak power step. Therefore, the GE correlation which did well at the peak power location would again be expected to do well. In the upper section. of the core, where few critical heat fluxes occurred, the GE correlation calculated that a boiling crisis would occur (Figure 19). A CHF was calculated because the calculated quality was about the same as in the center and lower 


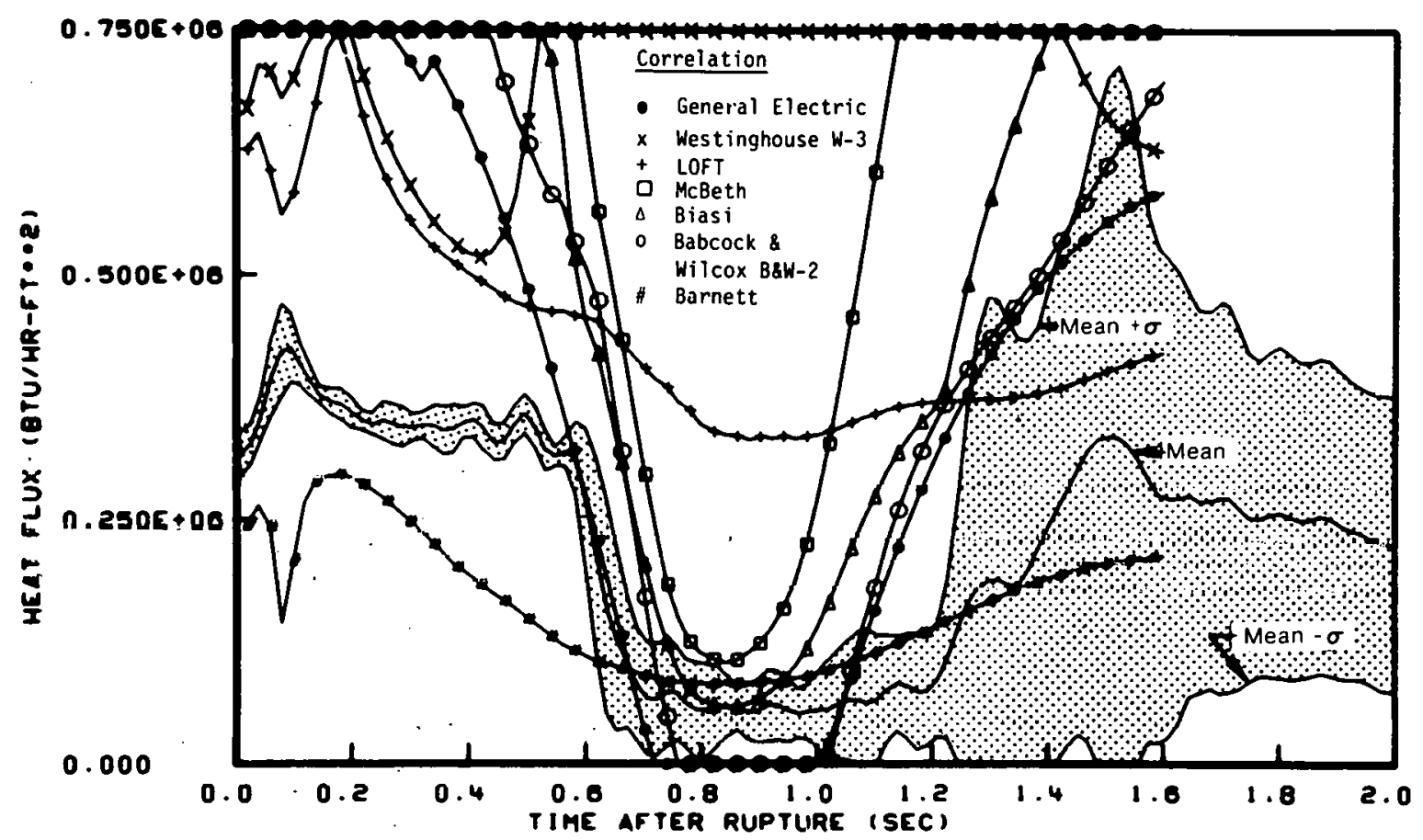

Fig. 18 Calculated critical heat fluxes compared with the mean heat flux in the lower part of the core (11 to 16 in).

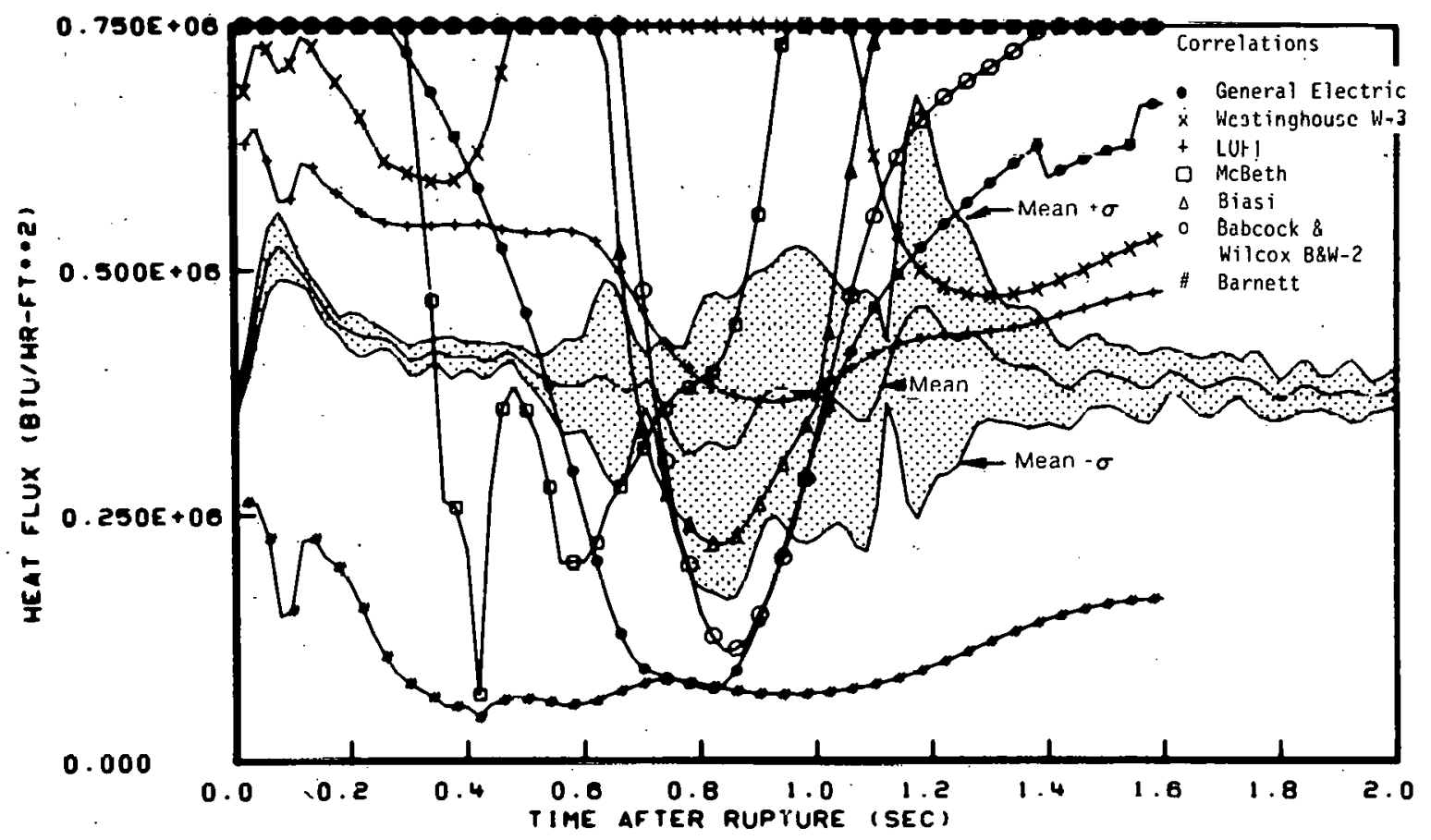

Fig. 19 Calculated critical heat fluxes compared with the mean heat flux in the upper part of the core ( 31 to 36 in). 
parts of the core. Therefore, the GE correlation (using the COBRA-IV-I calculated hydraulic conditions) provided a conservative calculation of the CHF in the upper part of the core.

The results for the lower section of the core from the MacBeth, Biasi, and B\&W-2 correlations were similar to those for the peak power level. These correlations calculated close to the same time for a boiling crisis, which was about $100 \mathrm{msec}$ after the mean heat flux began to decrease. In the upper part of the core, these three correlations calculated that a CHF also would have happened at this core elevation. The curve for the Westinghouse W-3 correlation remained larger than the mean heat flux curve at the 11- to 16-in. level (Figure 19), thus calculating no CHF. Above the peak power step, where few rods experienced CHF, the W-3 correlation also calculated that no boiling crisis would happen. The LOFT correlation did not calculate that a CHF would be reached in the lower part of the core (Figure 18) but did calculate that a CHF would occur above the center of the core (Figure 19). The Barnett correlation calculated CHF values which were below the mean heat flux curve in both the upper and lower core before and after rupture as shown in Figures 18 and 19.

The preceding results have shown that the time of CHF could be calculated well using an existing CHF correlation. This capability to calculate CHF resulted primarily from the fact that those correlations which did well strongly depended on fluid quality and that during the time of $\mathrm{CHF}$, the quality rose quickly and to a large value. Because of the fast increase in quality within the core, a large variation in thermal-hydraulics had only minor effects on the calculated time for CHF. Figure 20 shows that the times calculated by the GE-CHF correlation for CHF at the peak power step using the wide range in quality shown in Figure 21 were within $100 \mathrm{msec}$ of each other.

Therefore, the conclusion reached is that existing CHF correlations can be used to calculate the time of CHF in the Semiscale core adequately during a $200 \%$ cold leg break simulation. Specific conclusions were:

(1) The excellent calculation capability of the General Electric Company correlation and the good capability of the MacBeth, Biasi, and B\&W-2 correlations for the Semiscale core indicate that these correlations are adequate for blowdown analysis in Semiscale and that no new CHF calculation methods are needed for the Semiscale core

(2) The modifications to the steady state correlations which needed modification to be applicable to blowdown with reverse flow were not sufficient to provide an adequate calculation of CHF in the Semiscale core. 


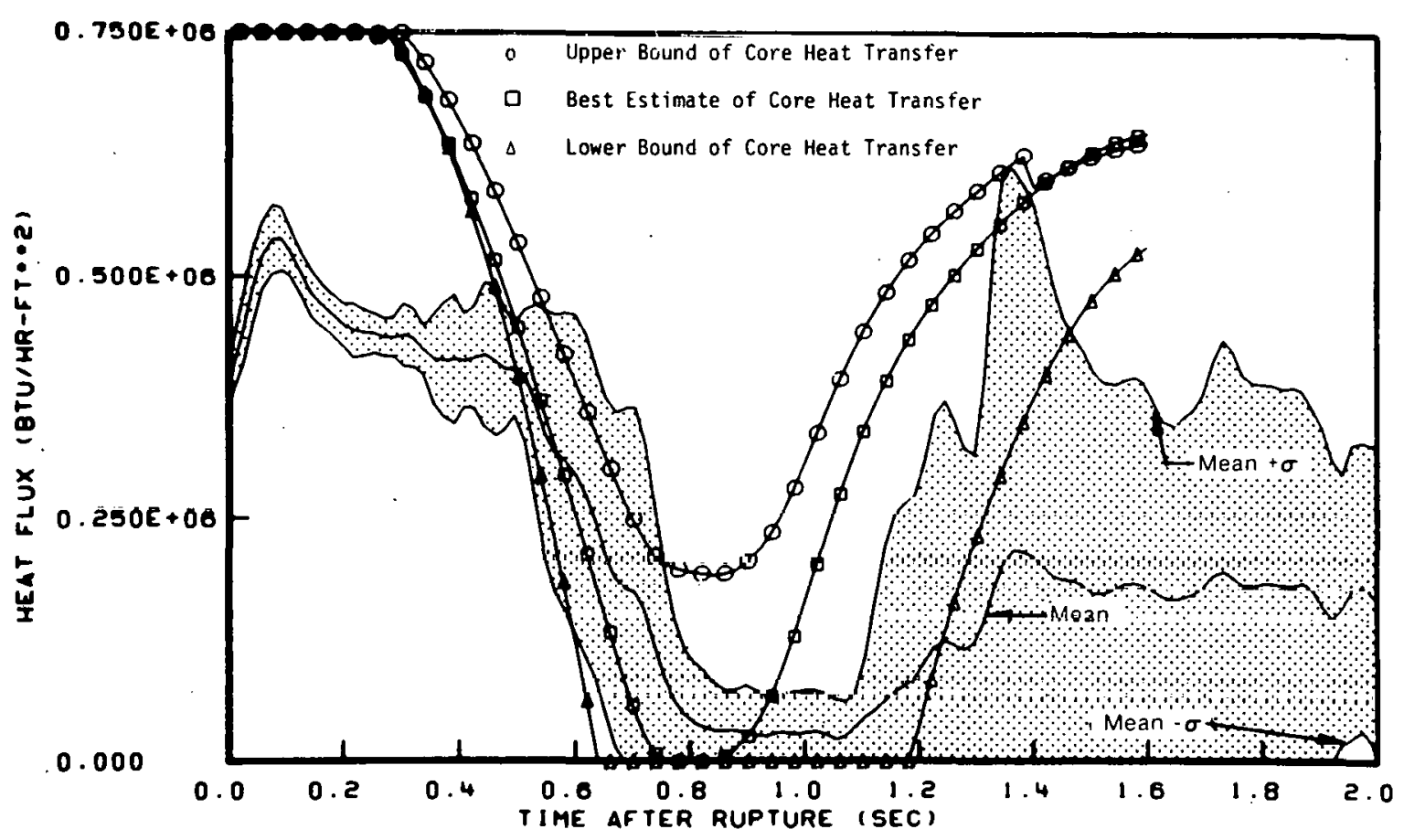

Fig. 20 Variation in CHF from the General Electric Corporation correlation using hydraulics calculated from COBRA-IV-I and the bounds in core heat transfer.

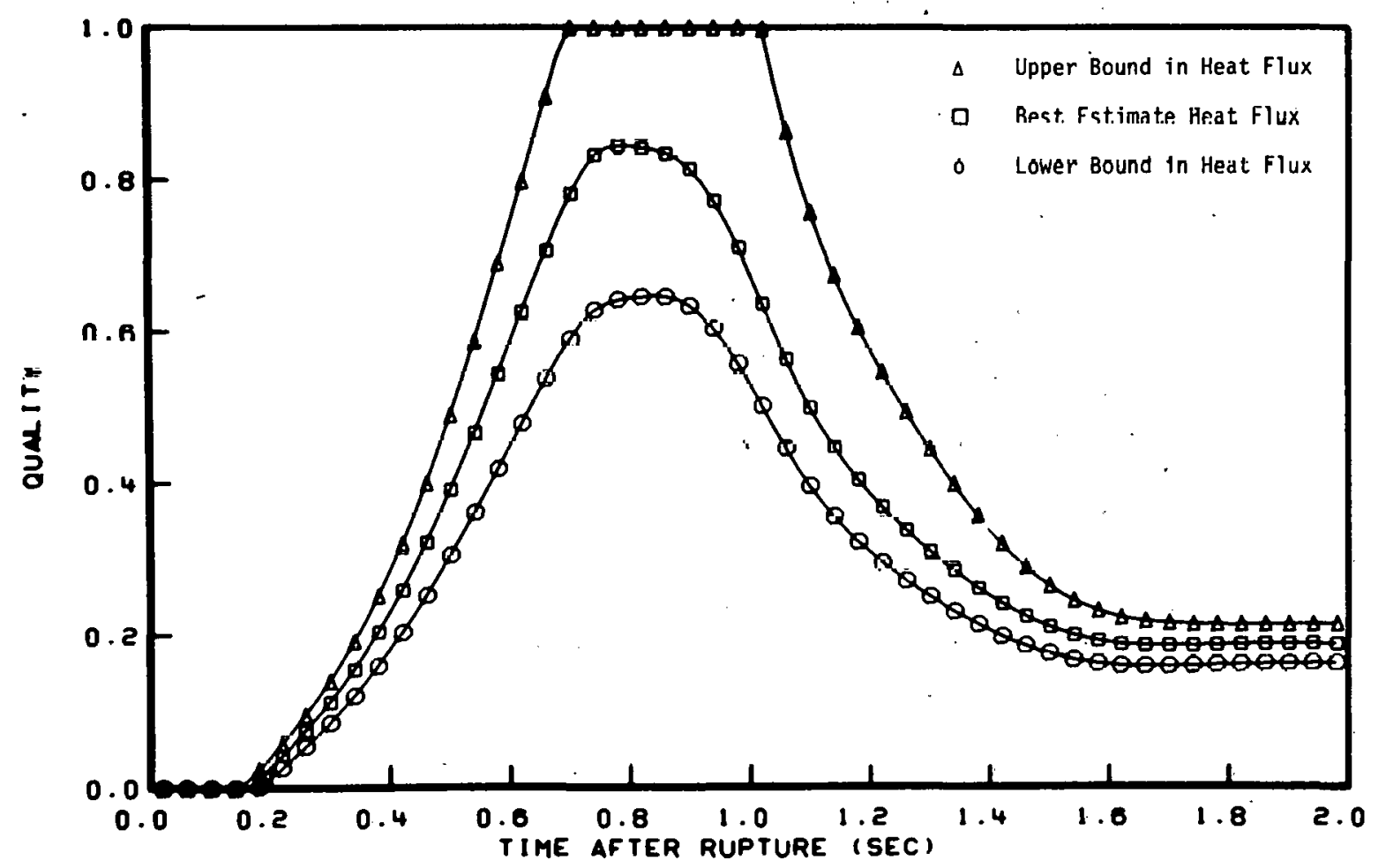

Fig. 21 Variation in COBRA-IV-I calculated quality from the uncertainty in core heat transfer rate. 


\title{
II. LOFT EXPERIMENTAL PROGRAM
}

\author{
L. P. Leach, Acting Manager
}

A test program and study to determine the effects on the departure from nucleate boiling (DNB) phenomena in the Loss-of-Fluid Test (LOFT) core, which has thermocouples mounted on the outside surfaces of the fuel rods, during steady state operating conditions were completed. An experimental transit time flowmeter system was also completed.

\section{EFFECTS OF FUEL ROD SURFACE THERMOCOUPLES}

ON STEADY STATE DEPARTURE FROM

NUCLEATE BOILING PHENOMENA

R. P. Wadkins and T. M. Howe

- A test program to determine the effect thermocouples mounted on the outside surface of fuel rods would have on the departure from nucleate boiling phenomena in the LOFT core during steady state operating conditions was completed. From the DNB test data, a correlation was developed for use in predicting DNB heat fluxes in the LOFT core.

\subsection{DNB Test Program \\ R. C. Gottula}

The LOFT in-core experiment instrumentation includes thermocouples with a 1.17-mm-OD sheath attached to the outside surfaces of the fuel rod cladding by laser welding. An extensive steady state DNB test program was conducted in the heat transfer facility at the Chemical Engineering Laboratories of Columbia University to determine the effects of the thermocouples. on DNB phenomena in the LOFT core for steady state conditions. Two 25-rod electrically heated bundles, one with and one without simulated external thermocouples on the rods, were tested. The rod bundle configurations are shown in Figures 22 and 23, and the bundle characteristics are given in Table I. The experiment parametcrs investigated were:

(1) Pressures from 6.9 to $16.5 \mathrm{MPa}$

(2) Inlet mass velocities from 1020 to $3400 \mathrm{~kg} / \mathrm{s}-\mathrm{m}^{2}$

(3) Local quality at DNB from -0.35 to +0.53 . 


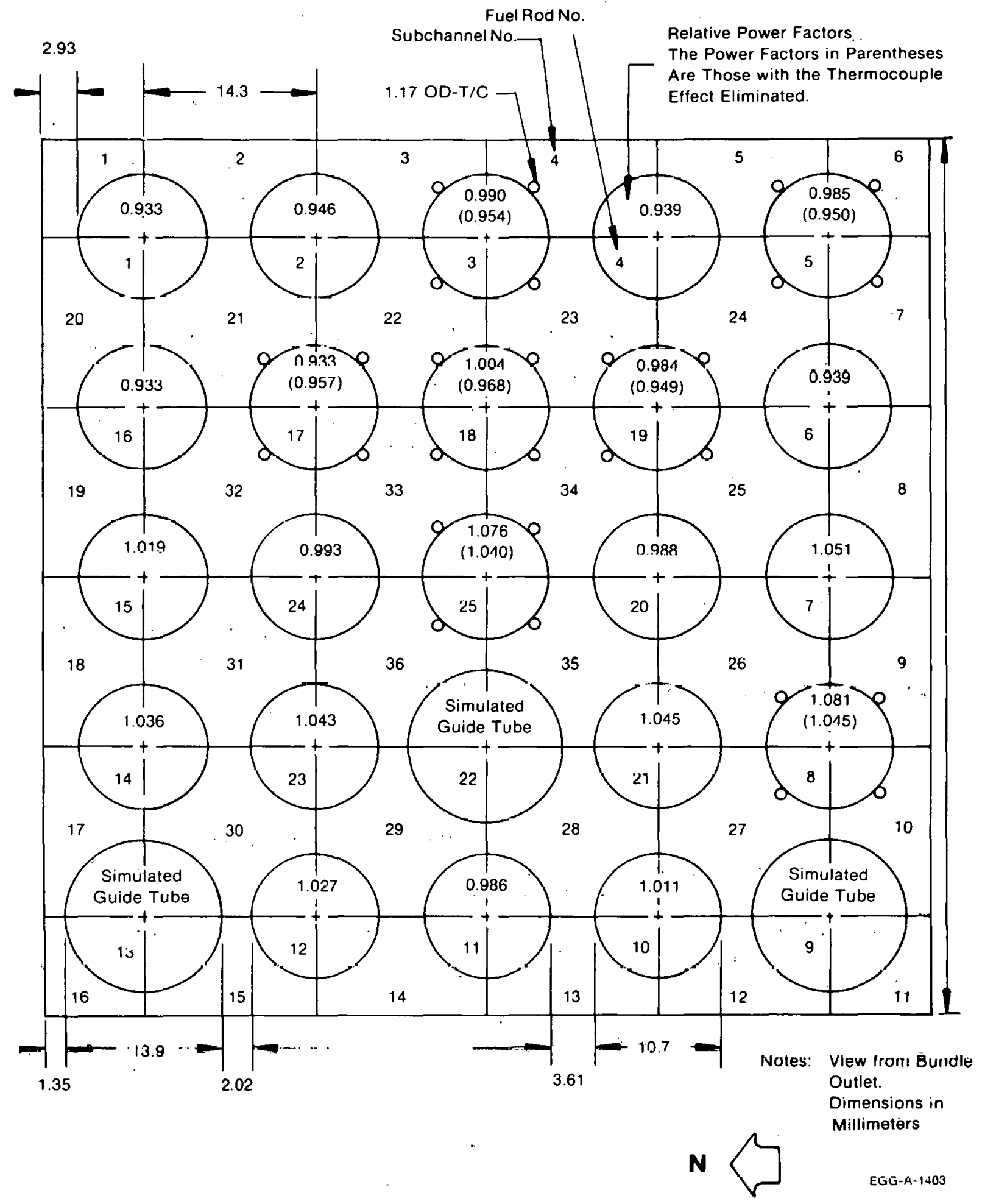

Fig. 22 Layout for bundle with external thermocouple simulators. 


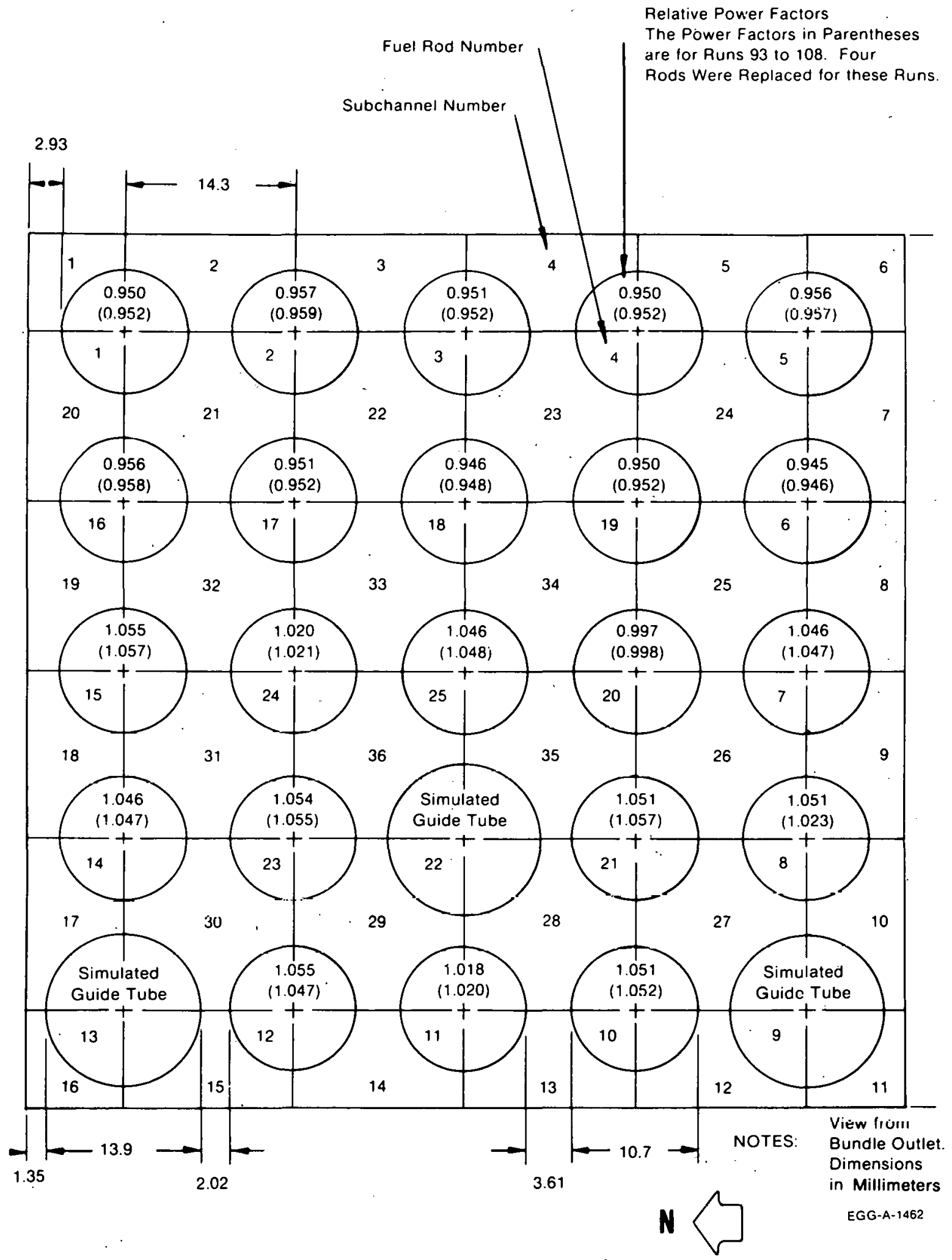

Fig. 23 Layout for bundle without cxtcrnal thermocouiple simulators. 
ROD BUNDLE CHARACTERISTICS

\begin{tabular}{lc}
\hline Number of rods & 25 \\
Number of unheated rods & 3 \\
Array & $5 \times 5$ \\
Heated length & $1.68 \mathrm{~m}$ \\
Pitch & $14.3 \mathrm{~mm}$ \\
Heated rod outside diameter & $10.7 \mathrm{~mm}$ \\
Unheated rod outside diameter & $13.9 \mathrm{~mm}$ \\
Axial heat flux distribution & unitorm \\
Radial heat flux distribution & nonuniform \\
Number of grid spacers & 5 \\
(LoFT-type, no mixing vanes) & \\
Grid spacing & $420 \mathrm{~mm}$ \\
Number of simulated external thermocouples \\
on rods with thermocouples \\
Simulated thermocouple outside diameter \\
Square flow channel dimension
\end{tabular}

Data from 455 data points were obtained using the rod bundle with thermocouple simulators mounted externally on the rods. These data were compared with data from 141 data points obtained with the rod bundle without external thermocouple simulators. Comparison of data in Figure 24 with data in Figure 25 indicates that the DNB heat tlux is higher for the bundle without thermocouple simulators than for the bundle with the simulators both at steady state and over the following operating ranges:

(1) Pressures from 13.8 to $16.5 \mathrm{MPa}$

(2) Mass velocities from 1360 to $3400 \mathrm{~kg} / \mathrm{s}-\mathrm{m}^{2}$

(3) Qunlity from -0.22 to +0.20 .

Since the DNB heat flux was lower for the bundle with external thermocouple simulators, only the points obtained with the external thermocouple simulators were used in the I.OFT DNB correlation. Although the steady state DNB tests indicated a difference between the test bundles with and those without the external thermocouple simulators, further testing must be performed to determine if the rod external thermocouples affect DNB under blowdown conditions similar to those expected in LOFT during loss-of-coolant experiments. A series of transient DNB tests will be performed at Columbia University on rod bundles similar to those used for the steady state DNB test program. 


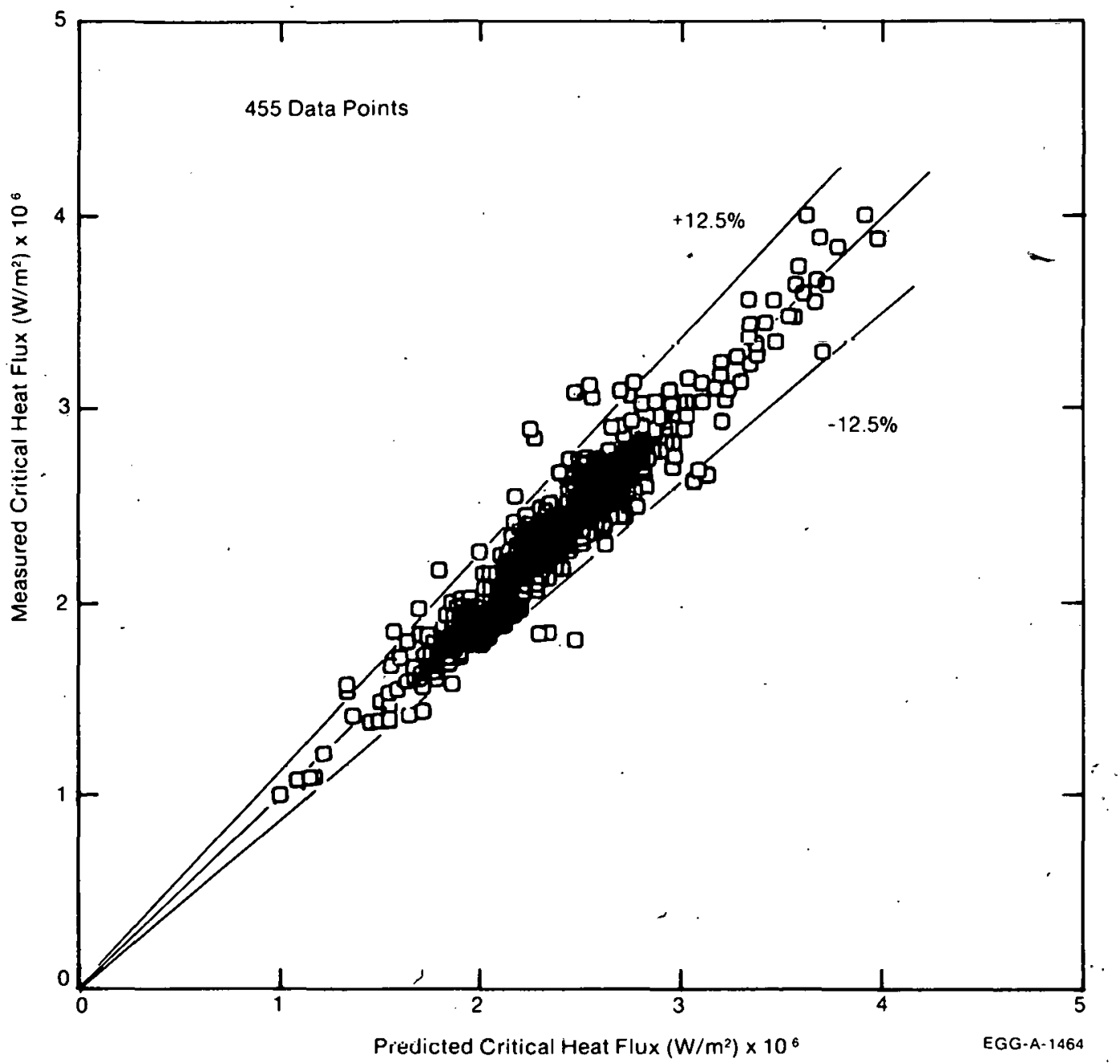

Fig. 24 Comparison of measured and predicted critical heat flux (LOFT + COBRA-II) for LOFT bundles with external thermocouple simulators.

\subsection{LOFT DNB Correlation}

\section{S. A. Fire and R. C. Gottula}

Attempts to predict the data from the DNB tests with the rod bundle with thermocouple simulators mounted on the outside walls of the rods were unsuccessful using existing DNB correlations ${ }^{[11]}$. Therefore, the following DNB correlation was developed for application to the LOFT core:

$$
q_{D N B}=269.29 G+365.73 P-0.018196 P^{2}-3343.3 G X+0.086510 G P X
$$

where

$$
\begin{aligned}
& \mathrm{q}=\text { DNB heat flux }\left(\mathrm{W} / \mathrm{m}^{2}\right) \\
& \mathrm{G}=\text { mass velocity }\left(\mathrm{kg} / \mathrm{s}-\mathrm{m}^{2}\right)
\end{aligned}
$$




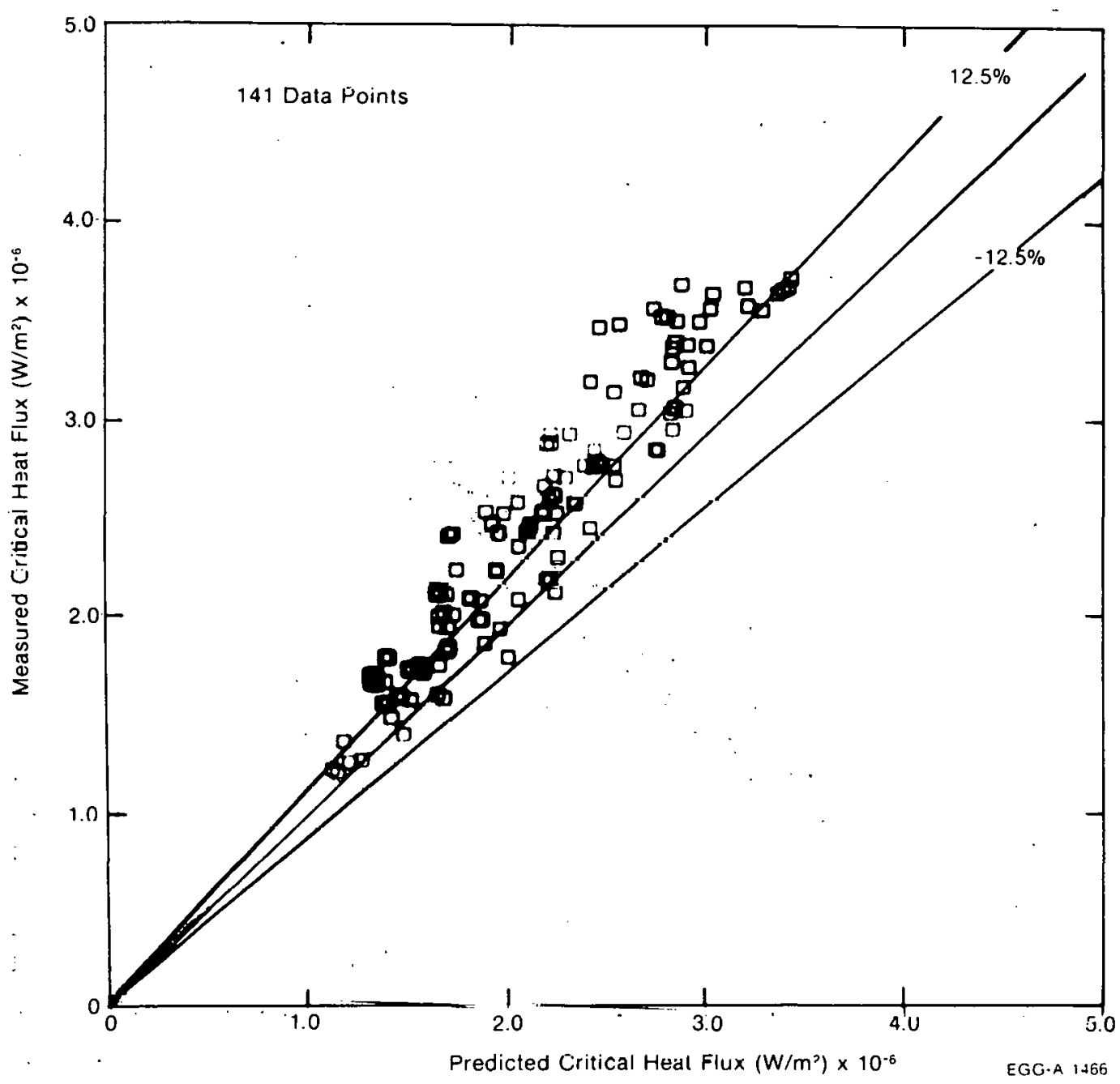

Fig. 25 Comparison of measured and predicted criticãl neat flux (LOFT I CODRA II) for LOFT hịındies withnut external thermocouple simulators.

$$
\begin{aligned}
& \mathrm{P}=\text { pressure (kPa) } \\
& \mathrm{X}=\text { local quality (fraction). }
\end{aligned}
$$

The upplioation range of the LOFT correlation is the tested range given in Section II-1.1. The LOFT DNB correlation predicts the DNB heat flux for the 455 data points from the DNB tests. with a mean and standard deviation of 0.9998 and 0.0645 , respectively. Figure 26 slıuws a histogram giving the frequency distribution of the ratio of measured to predicted DNB heat fluxes for the source data and the corresponding normal distribution curve. Figure 24 shows a comparison between the measured DNB heat fluxes from the test and the DNB heat fluxes predicted with the LOFT DNB correlation.

The LOFT DNB correlation will be used to predict thermal safety margins for the LOFT reactor steady state operating conditions and for plant transient conditions other than loss-of-coolant experiments. The LOFT correlation with a minimum DNB ratio of 1.13 provides a $95 \%$ probability at a $95 \%$ confidence level of not experiencing DNB.on a hot rod. 


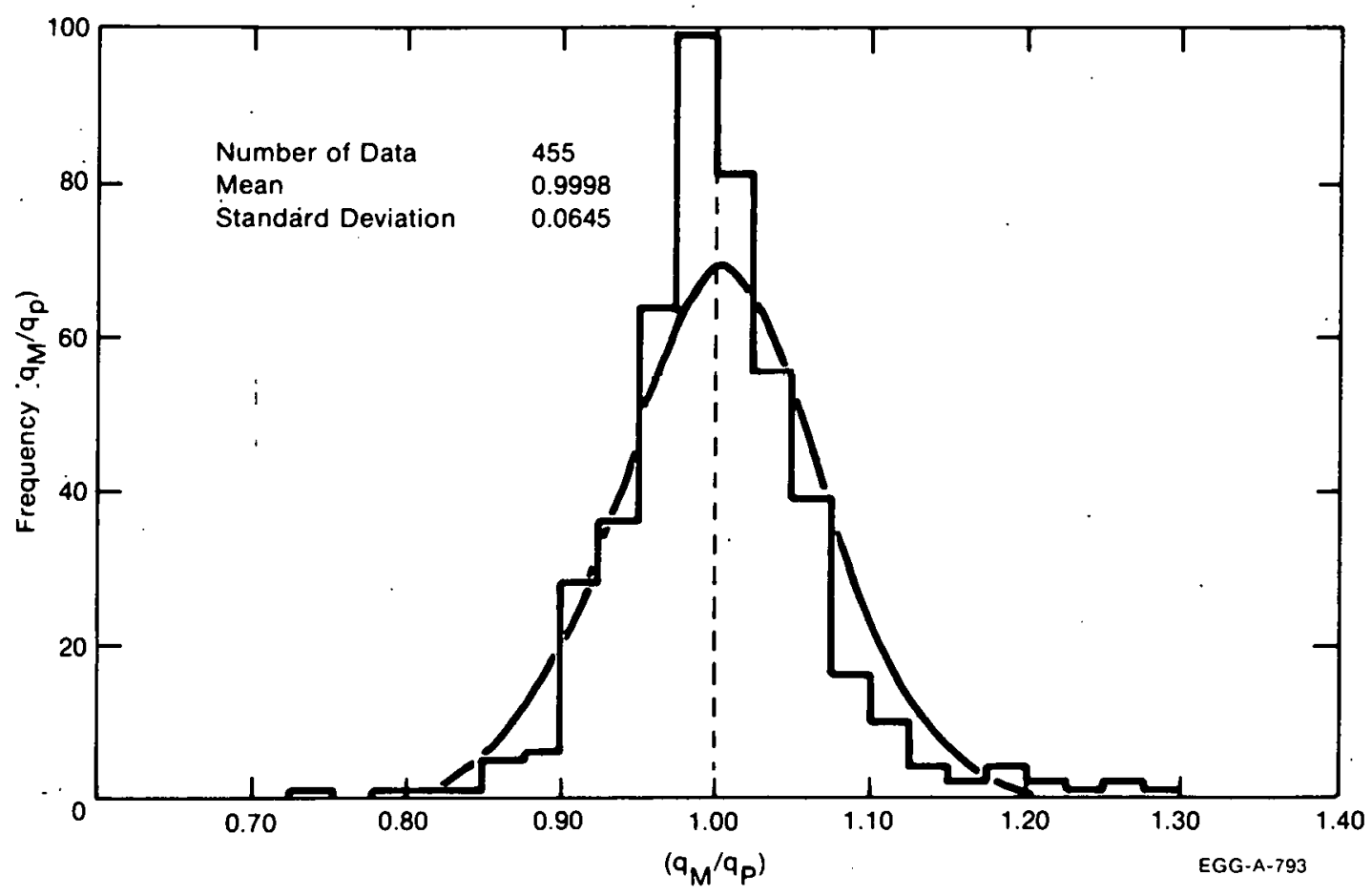

Fig. 26 Histogram of ratio of measured-to-predicted.critical heat fluxes for the LOFT correlation and source data.

\section{TRANSIT TIME FLOWMETER DEVELOPMENT}

G. D. Lassahn

Preliminary results of a study to determine the feasibility of using transit time flowmeters (TTF) in LOFT experiments have been obtained. Tests of an experimental TTF system using thermocouples as sensors and a Princeton Applied Research Corporation correlator as a data analysis system have been completed. The primary conclusion drawn from these tests is that the thermocouple signals do not have enough correlated high frequency power to allow an acceptably low level of statistical error in the transit time determination.

The study is being continued in two areas: (a) a basic theoretical study of the conditions (signal fiequency content, averaging time, correlation magnitude, etc.) necessary for a given accuracy in a TTF, and (b) experimental work to determine the characteristics of signals from various sensors (drag screens, ultrasonic void sensors, electrical conductivity void sensors, and gamma densitometers) in steam-water blowdown conditions. 
Preliminary results obtained from the first part of the theoretical study are shown in Figure 27. Figure 27 indicates how the probable error in the position of the correlation peak $\left(\sigma_{\tau}\right)$ depends on the averaging time or record length $(\mathrm{T})$ for different correlation peak magnitudes $(\rho)$. It is assumed that the correlated power is uniformly distributed over the angular frequency range between 0 and $\omega$. If the uncorrelated power is also uniformly distributed over this range, the error is indicated by the line along the bottom edge of the shaded band with the appropriate value of $\rho$. If the total power is uniformly distributed between angular frequencies 0 and $\omega_{1}$, with $\omega_{1}>\omega$, and the correlation is perfect for frequencies below $\omega$ but zero for frequencies above $\omega$, then the line along the top edge of the shaded band indicates the error. Intermediate distributions of the uncorrelated power correspond to intermediate positions in the shaded band.

If, for example, $\omega=60$ and $\rho=0.5$ (approximately representative of thy thermocouples used in the previously mentioned experiments), the error in the transit time determination is shown to be between 11 and $17 \mathrm{~ms}$, depending on the spectral distribution of the uncorrelated power, with a record length of $1 \mathrm{~s}(\mathrm{~T}=1)$. With a 20 -s recond length, the error drops to between 2.5 and $3.8 \mathrm{~ms}$.

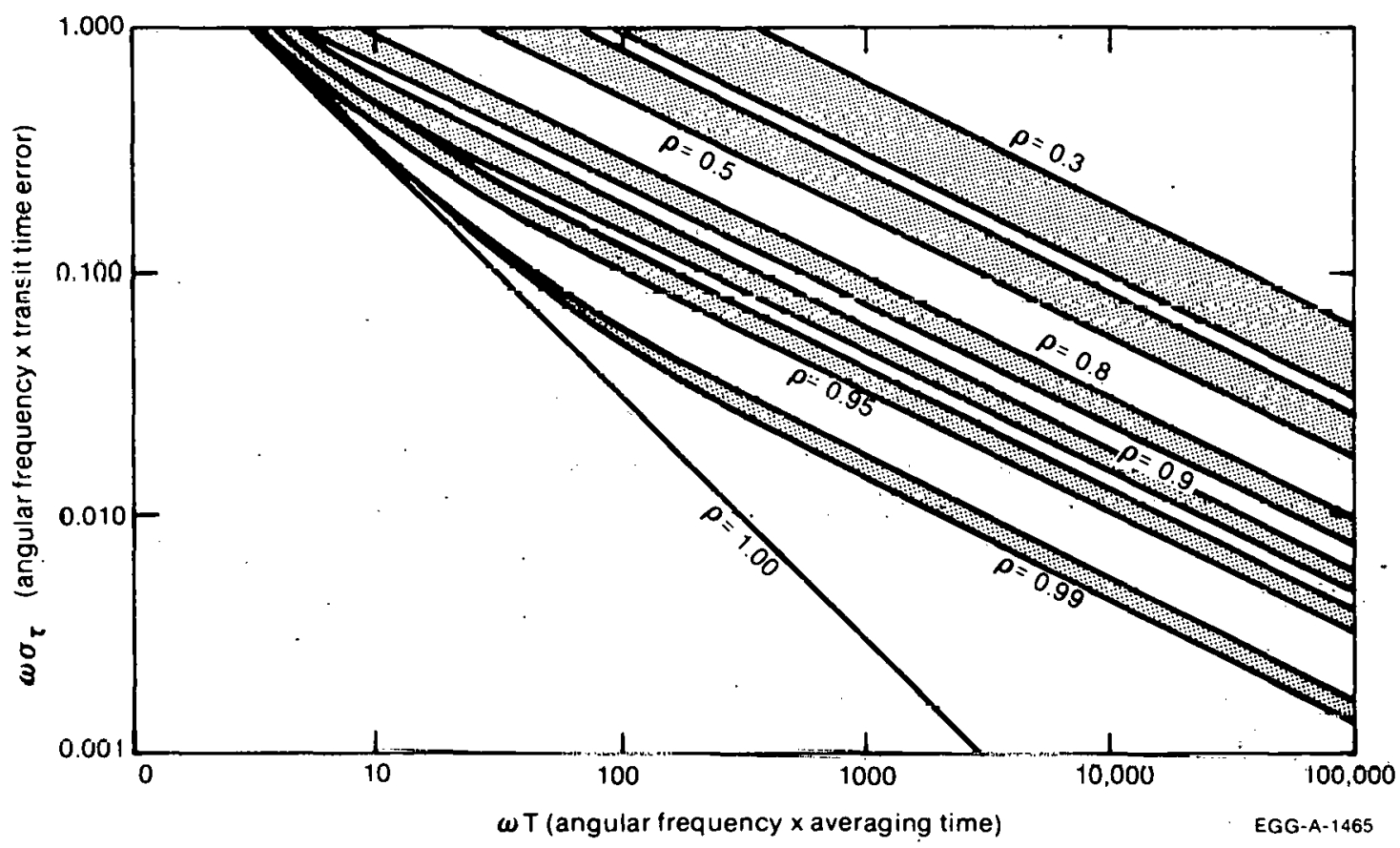

Fig. 27 Transit time standard deviation. 


\title{
III. THERMAL FUELS BEHAVIOR PROGRAM
}

\author{
J. O. Zane, Manager
}

Tests PCM-4, IE-2, IE-3, and GC 2-1 were conducted in the Power Burst Facility (PBF) Reactor. The following sections describe (a) preliminary results from these tests plus preliminary results of postirradiation examination of fuel rods from Test IE-1, which was discussed previously ${ }^{[13]}$; (b) thermal fuels behavior program development and evaluation; and (c) fuel model development and verification.

\section{PBF TESTING}

E. E. Burdick

Four nuclear tests, PCM-4, IE-2, IE-3, and GC 2-1, were conducted in the PBF. Postirradiation examinations were conducted on fuel rods from Test IE-1, previously conducted in the PBF ${ }^{[6]}$.

\subsection{Power-Cooling-Mismatch (PCM) Test PCM-4 Preliminary Results}

Z. R. Martinson and S. A. Ploger

The eighth test (PCM-4) in the PCM test series was completed. The objective of Test PCM-4 was to obtain fuel rod behavior data during film boiling operation for representative pressurized water reactor (PWR) conditions. Maximum cladding surface temperatures from 810 to $1255 \mathrm{~K}$ were sought. This temperature range includes the $\alpha$ phase and $\alpha+\beta$ phase of zirconium. The objectives and preliminary results of previous related PCM tests, PCM-2 and PCM-3, were presented earlier ${ }^{[6,13]}$. The preliminary results of Test PCM-4 are given in the following subsections.

1.1.1 Test Configuration. Test PCM-4 was performed with four PWR-type fuel rods designated UTA-0014, UTA-0015, UTA-0016, and A-0017. Each rod was contained in a separate flow shroud and located in the PBF in-pile tube (IPT) as shown in Figure 28. Some basic dimensions and details of the fuel rods are as follows:

$\begin{array}{lc}\text { Cladding material } & \text { zircaloy-4 } \\ \text { Cladding OD } & 10.7 \mathrm{~mm} \\ \text { Cladding ID } & 9.5 \mathrm{~mm} \\ \text { Fuel pellet OD } & 9.3 \mathrm{~mm} \\ \text { Pellet density } & 93 \% \text { theoretical } \\ \text { U-235 enrichment } & 20 \%\end{array}$




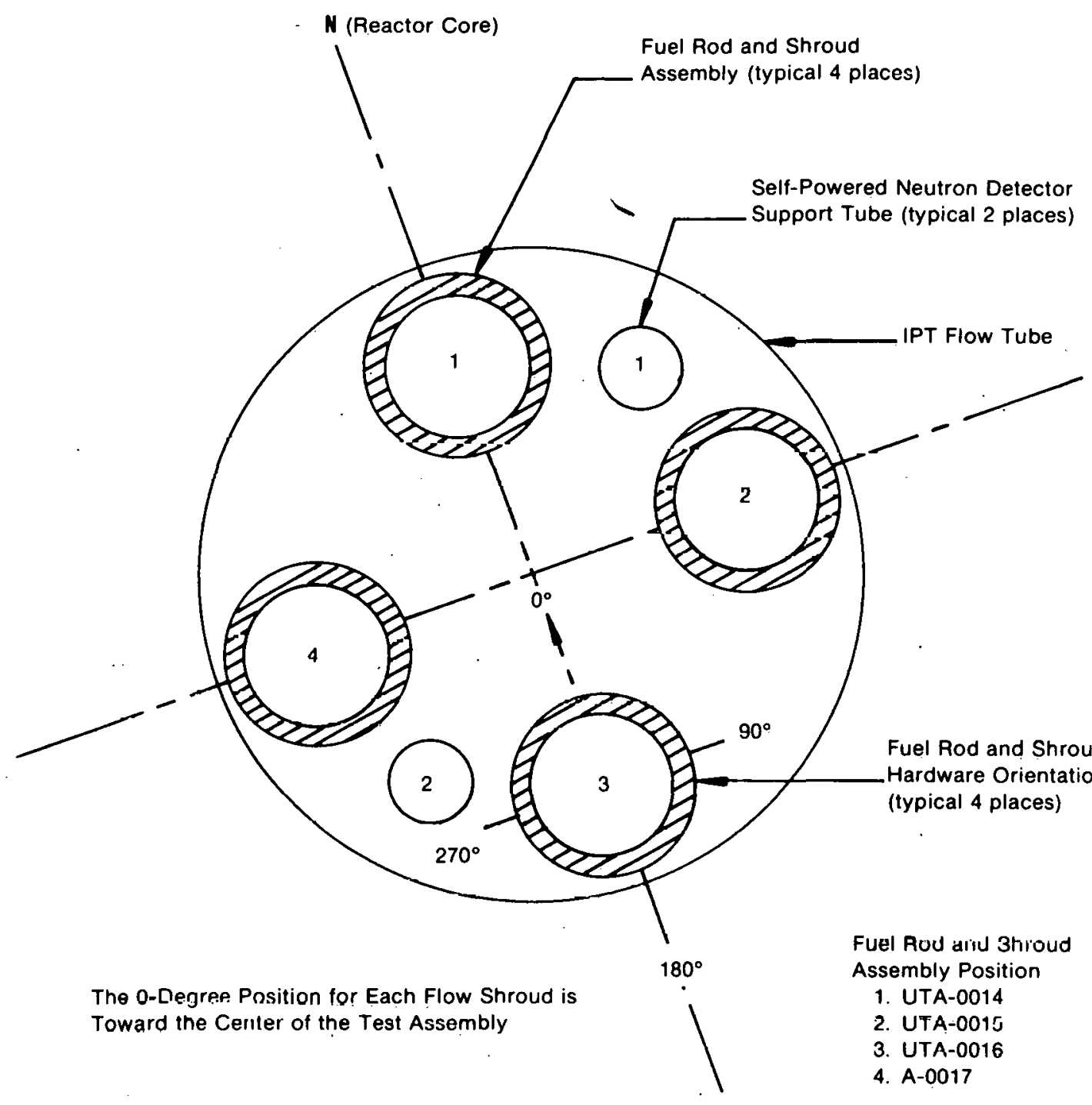

EGG-Aं-1476

Fig. 28 Top viow of PCM-4 fuel rnd lncations in the PBF in-pile tube.

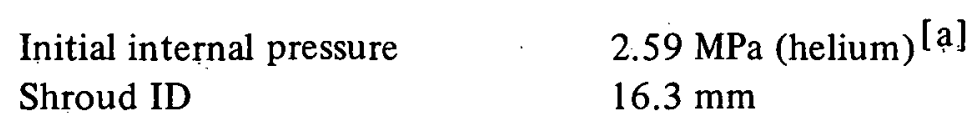

Three ol the fucl rods wert iristrumentr.d for measurement of the internal gas pressure, fuel centerline temperature, and cladding surface temperature. The change in cladding axial length was measured on all four rods. Test conditions, such as coolant volumetric flow rate for each rod, temperature rise, coolant temperature, and system pressure, were also mcasured.

[a] $3.79 \mathrm{MPa}$ for Rod A-0017 only. 
1.1.2 Departure from Nucleate Boiling (DNB) Testing. After $20 \mathrm{~h}$ steady state reactor operation to measure the fuel rod powers and to precondition the fuel rods, DNB testing was initiated. The initial nominal coolant temperature, pressure, and mass flux were $600 \mathrm{~K}, 15.1 \mathrm{MPa}$, and $2200 \mathrm{~kg} / \mathrm{s} \cdot \mathrm{m}^{2}$ respectively ${ }^{[a]}$. The fuel rod peak powers ${ }^{[b]}$ were increased from 4 to $72 \mathrm{~kW} / \mathrm{m}$ at an average rate of $4 \mathrm{~kW} / \mathrm{m}$ per minute. As the rod powers reached $72 \mathrm{~kW} / \mathrm{m}$, DNB occurred on all four fuel rods. The maximum cladding surface temperature, $729 \mathrm{~K}$, was indicated by the $0.635-\mathrm{m}$ elevation thermocouple on Rod UTA-0015. The test rod powers were decreased to $58 \mathrm{~kW} / \mathrm{m}$ to eliminate DNB conditions. Later, the coolant mass flux was increased to $3000 \mathrm{~kg} / \mathrm{s} \cdot \mathrm{m}^{2}$, then the fuel rod powers were increased to $63 \mathrm{~kW} / \mathrm{m}$. After operating under these conditions for $1 \mathrm{~h}$ to "age" the fuel rods, four coolant flow reduction cycles were performed. DNB conditions were achieved by reducing the coolant flow in small increments until DNB was indicated. The coolant flow was held constant for about $1 \mathrm{~min}$ after each flow reduction. The test rod powers were held constant during each flow reduction. DNB Cycles 1,2, and 4 were performed at fuel rod peak powers of $69 \mathrm{~kW} / \mathrm{m}$, while the fuel rod peak powers for DNB Cycle 3 were $60 \mathrm{~kW} / \mathrm{m}$. Only during the fourth (and final) DNB cycle were the indicated cladding temperatures allowed to reach high values (greater than $740 \mathrm{~K}$ ). Shortly after DNB occurred in Cycles 1 and 2, the fuel rod powers were decreased and coolant mass flux increased to about $3000 \mathrm{~kg} / \mathrm{s} \cdot \mathrm{m}^{2}$. DNB was eliminated in DNB Cycle 3 by increasing the coolant mass flux while the rod powers were held constant at $60 \mathrm{~kW} / \mathrm{m}$.

A summary of the experiment results obtained during the DNB testing is given in Table II. Rods UTA-0015 and A-0017 reached DNB in Cycles 1 and 2, but only Rod UTA-0015 reached DNB during Cycle 3. All four of the rods reached DNB conditions during the final DNB cycle. The fuel rods were allowed to remain in film boiling conditions for about $160 \mathrm{~s}$ before the reactor was scrammed. Maximum cladding surface temperatures of 930,1100 , and $1005 \mathrm{~K}$ were measured for Rods UTA-0014, UTA-0015, and UTA-0016, respectively.

1.1.3 Posttest Examination. Rods UTA-0014, UTA-0015, UTA-0016, and A-0017 were withdrawn intact from their respective shrouds in a hot cell. Composite photographs of the posttest condition of the four fuel rods are shown in Figures 29 through 32.

[a] A coolant mass flux of $5775 \mathrm{~kg} / \mathrm{s} \cdot \mathrm{m}^{2}$ corresponds to a volumetric flow rate of $1 \mathrm{Q} / \mathrm{s}$ at $600 \mathrm{~K}$ and $15.1 \mathrm{MPa}$ system conditions for the instrumented fuel rods.

[b] All linear fuel rod powers $(\mathrm{kW} / \mathrm{m})$ quoted refer to the fuel rod peak power using an axial peak-to-average power ratio of 1.36 . The four fuel rods were producing nominally identical powers. 


\section{TABLE II}

SUMMARY OF EXPERIMENT RESULTS DURING DNB TESTING FOR -EST FCM-4

\begin{tabular}{|c|c|c|c|c|c|c|c|c|c|}
\hline $\begin{array}{c}\text { DNB } \\
\text { Cycle } \\
\text { Number } \\
\end{array}$ & $\begin{array}{c}\text { Tes: } \\
\text { Rod } \\
\text { Number } \\
\end{array}$ & $\begin{array}{c}\text { OMB } \\
\text { Occurrence } \\
\end{array}$ & $\begin{array}{l}\text { Fue] Rad } \\
\text { Peas Power } \\
\text { at DNE } \\
(\mathrm{k} w / \mathrm{m}) \\
\end{array}$ & 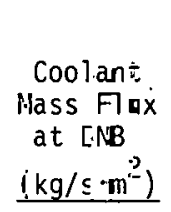 & $\begin{array}{r}\text { Mimimum } \\
\text { Coolant } \\
\text { Yass Flux. } \\
\left(\mathrm{kg} / \mathrm{s} \cdot \mathrm{m}^{2}\right) \\
\end{array}$ & $\begin{array}{c}\text { Coolant } \\
\text { Inlet } \\
\text { Temperature } \\
\text { (K) } \\
\end{array}$ & $\begin{array}{l}\text { Maximum } \\
\text { Measured } \\
\text { Cladding } \\
\text { Temoerazure } \\
\text { (K) } \\
\end{array}$ & $\begin{array}{l}\text { Cladding }^{[\mathrm{a}]} \\
\text { Elongation } \\
(\mathrm{mm}) \\
\end{array}$ & $\begin{array}{l}\text { Increase in } \\
\text { Fuel Rod } \\
\text { Internal } \\
\text { Pressure } \\
\text { after DNB } \\
\text { (MPa) }\end{array}$ \\
\hline $\begin{array}{c}\text { Fuel rod } \\
\text { aging }\end{array}$ & $\begin{array}{r}\text { UTA-0J14 } \\
\text { UTA-0J15 } \\
\text { UTA-0016 } \\
\text { A-0.017 }\end{array}$ & $\begin{array}{l}\text { Yes } \\
\text { yes } \\
\text { hes } \\
\text { lies }\end{array}$ & $\begin{array}{l}72 \\
72 \\
.72 \\
72\end{array}$ & $\begin{array}{l}209 C \\
2150 \\
2064 \\
19 ? 3\end{array}$ & $\begin{array}{l}2007 \\
2104 \\
2036 \\
1940\end{array}$ & 603 & $\begin{array}{l}560 \\
729 \\
650 \\
{[a]}\end{array}$ & $\begin{array}{l}0.16 \\
0.8 \\
0.3 \\
0.5\end{array}$ & $\begin{array}{l}0 \\
0.06 \\
0.08 \\
{[\mathrm{~b}]}\end{array}$ \\
\hline 1 & $\begin{array}{r}\text { UTA-0014 } \\
\text { UTA-0015 } \\
\text { UTA-C016 } \\
\text { A-C017 }\end{array}$ & $\begin{array}{l}\text { vo } \\
\text { ves } \\
\text { No } \\
\text { Yes }\end{array}$ & $\begin{array}{l}69 \\
69 \\
69 \\
69\end{array}$ & $\begin{array}{c}21 \geq 5 \\
1973\end{array}$ & $\begin{array}{l}1906 \\
2010 \\
1929 \\
1906\end{array}$ & 600 & $\begin{array}{l}6 \vdots 1 \\
7 \vdots 1 \\
6 \vdots 3 \\
{[\bar{c}]}\end{array}$ & $\begin{array}{l}-- \\
0.4 \\
-- \\
0.2\end{array}$ & $\begin{array}{c}-- \\
0.09 \\
-\bar{b}]\end{array}$ \\
\hline 2 & $\begin{array}{r}\text { UTA-0014 } \\
\text { UTA-0015 } \\
\text { UTA-0016 } \\
\text { A-0017 }\end{array}$ & $\begin{array}{r}\text { No } \\
\text { res } \\
\text { No } \\
\text { yes }\end{array}$ & $\begin{array}{l}69 \\
69 \\
69 \\
69\end{array}$ & $\begin{array}{l}-- \\
2035 \\
\overline{1} \overline{5}\end{array}$ & $\begin{array}{l}1864 \\
1962 \\
1393 \\
1313\end{array}$ & 602 & $\begin{array}{r}632 \\
703 \\
.633 \\
{[a]}\end{array}$ & $\begin{array}{r}-- \\
0.9 \\
-- \\
0.4\end{array}$ & $\begin{array}{l}\overline{--} \\
0 . \overline{2} \\
{[\mathrm{~b}]}\end{array}$ \\
\hline 3 & $\begin{array}{r}\text { UTA-0014 } \\
\text { UTA-1)015 } \\
\text { UTA-1J016 } \\
\text { A- } 3017\end{array}$ & $\begin{array}{r}\text { No } \\
\text { Yes } \\
\text { No } \\
\text { No }\end{array}$ & $\begin{array}{l}50 \\
50 \\
60 \\
60 .\end{array}$ & 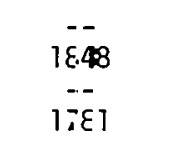 & $\begin{array}{l}1704 \\
1784 \\
1721 \\
1642\end{array}$ & 600 & $\begin{array}{l}630 \\
738 \\
631 \\
{[\mathrm{a}]}\end{array}$ & $\begin{array}{l}-- \\
0.1 \\
-- \\
0.02\end{array}$ & $\begin{array}{l}-- \\
0.06 \\
-- \\
{[b]}\end{array}$ \\
\hline 4 & $\begin{array}{r}\text { UTA-0014 } \\
\text { UTA-0015 } \\
\text { UTA-0016 } \\
\text { A-0017 }\end{array}$ & $\begin{array}{l}\text { ies } \\
\text { ies } \\
\text { ies } \\
\text { res }\end{array}$ & $\begin{array}{l}69 \\
69 \\
69 \\
69\end{array}$ & $\begin{array}{l}18 \vdots 2 \\
2090 \\
1832 \\
1354\end{array}$ & $\begin{array}{l}1532 \\
1660 \\
1603 \\
1512\end{array}$ & 602 & $\begin{array}{r}930 \\
7100 \\
7 C 05 \\
{[a]}\end{array}$ & $\begin{array}{l}1.1 \\
1.7 \\
1.8 \\
1.6\end{array}$ & $\begin{array}{l}0.24 \\
0.40 \\
0.38 \\
{[\mathrm{~b}]}\end{array}$ \\
\hline \multicolumn{10}{|c|}{ [a] Elongation after DNB occurret. } \\
\hline [b] Not & truniented. & & & & . & & . & & \\
\hline
\end{tabular}




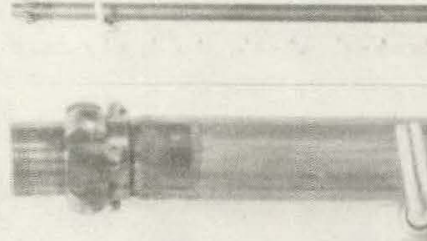

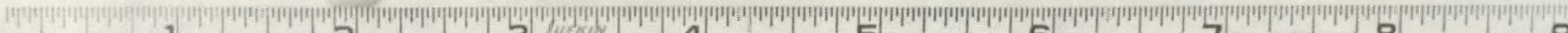

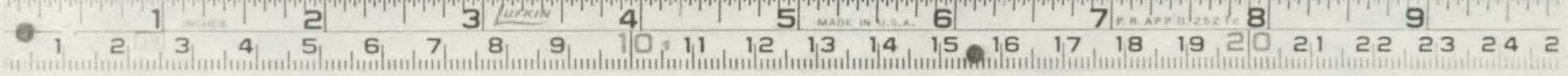

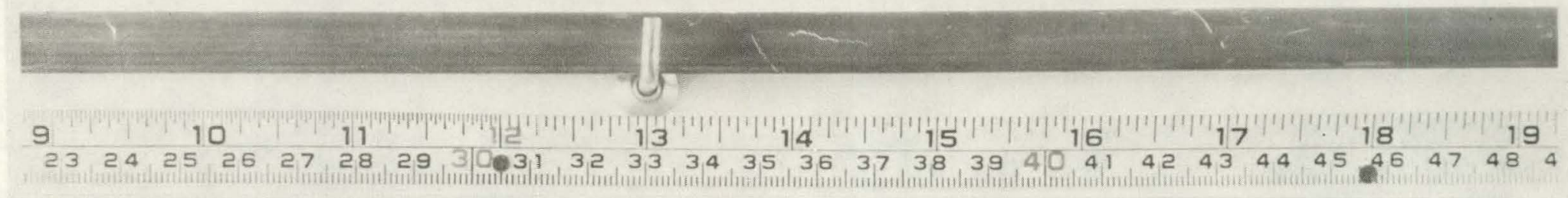

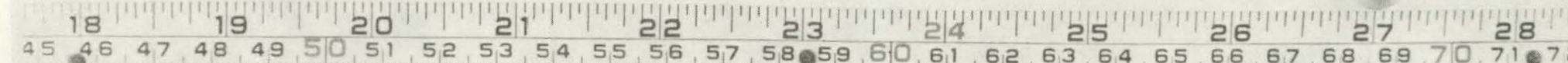

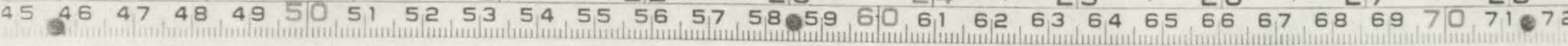

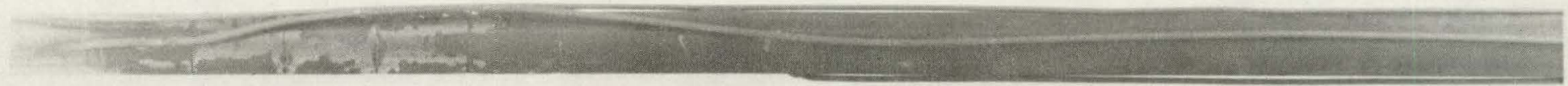

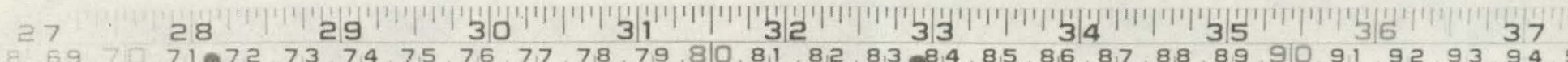

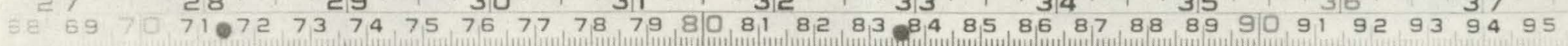

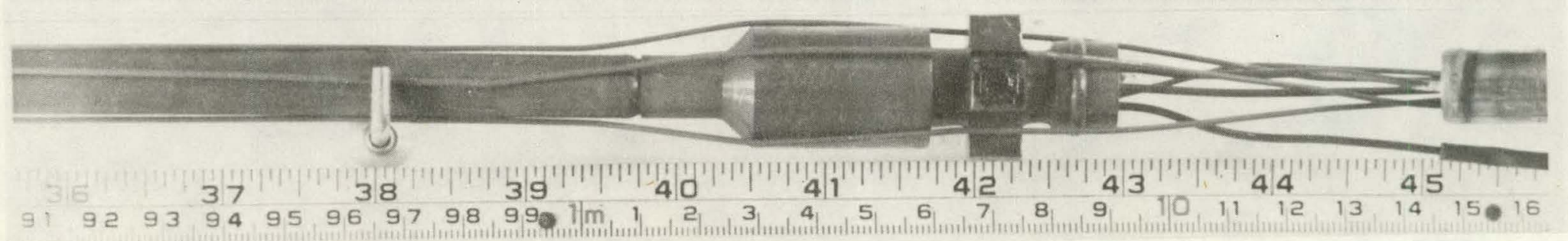

Fig. 29 Test PCM-4, Rod UTA-0014 $\left(0^{\circ}\right)$. 

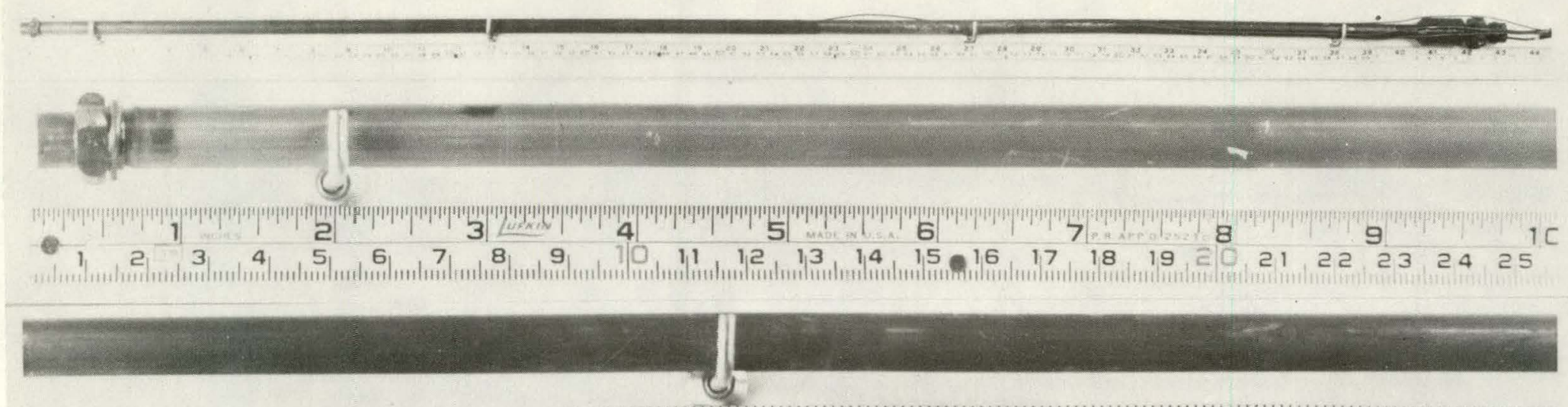

$9^{3}$

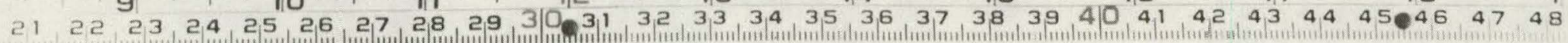

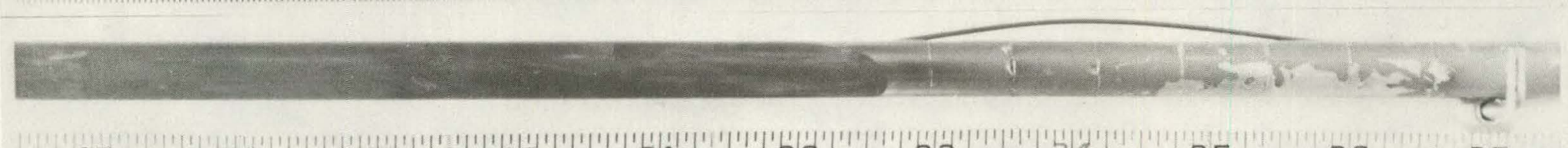

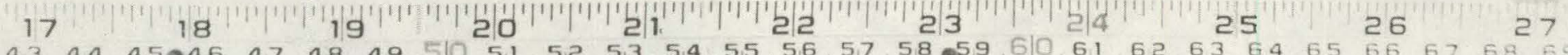

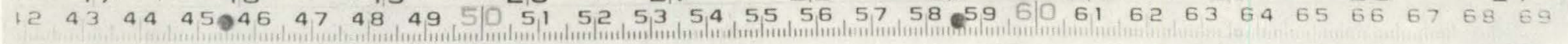

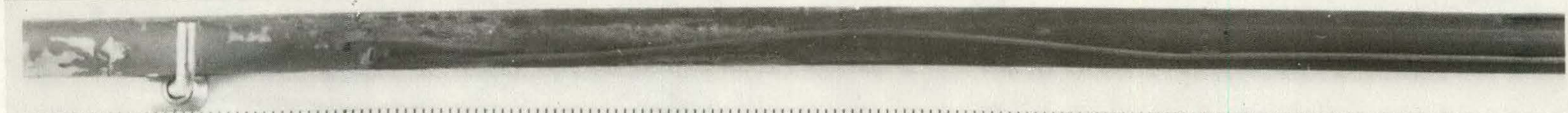

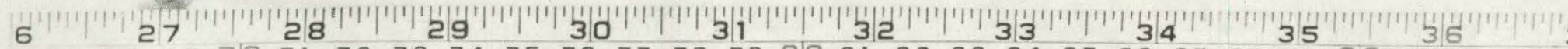

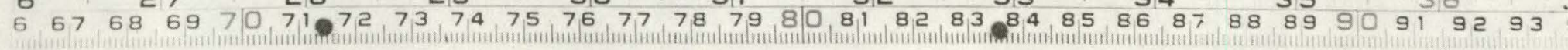

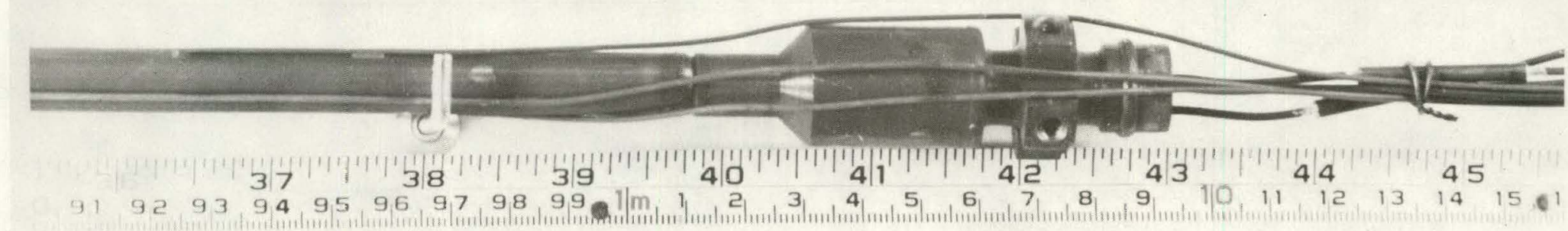

Fig. 30 Test PCM-4, Rod UTA-0015 $\left(180^{\circ}\right)$. 


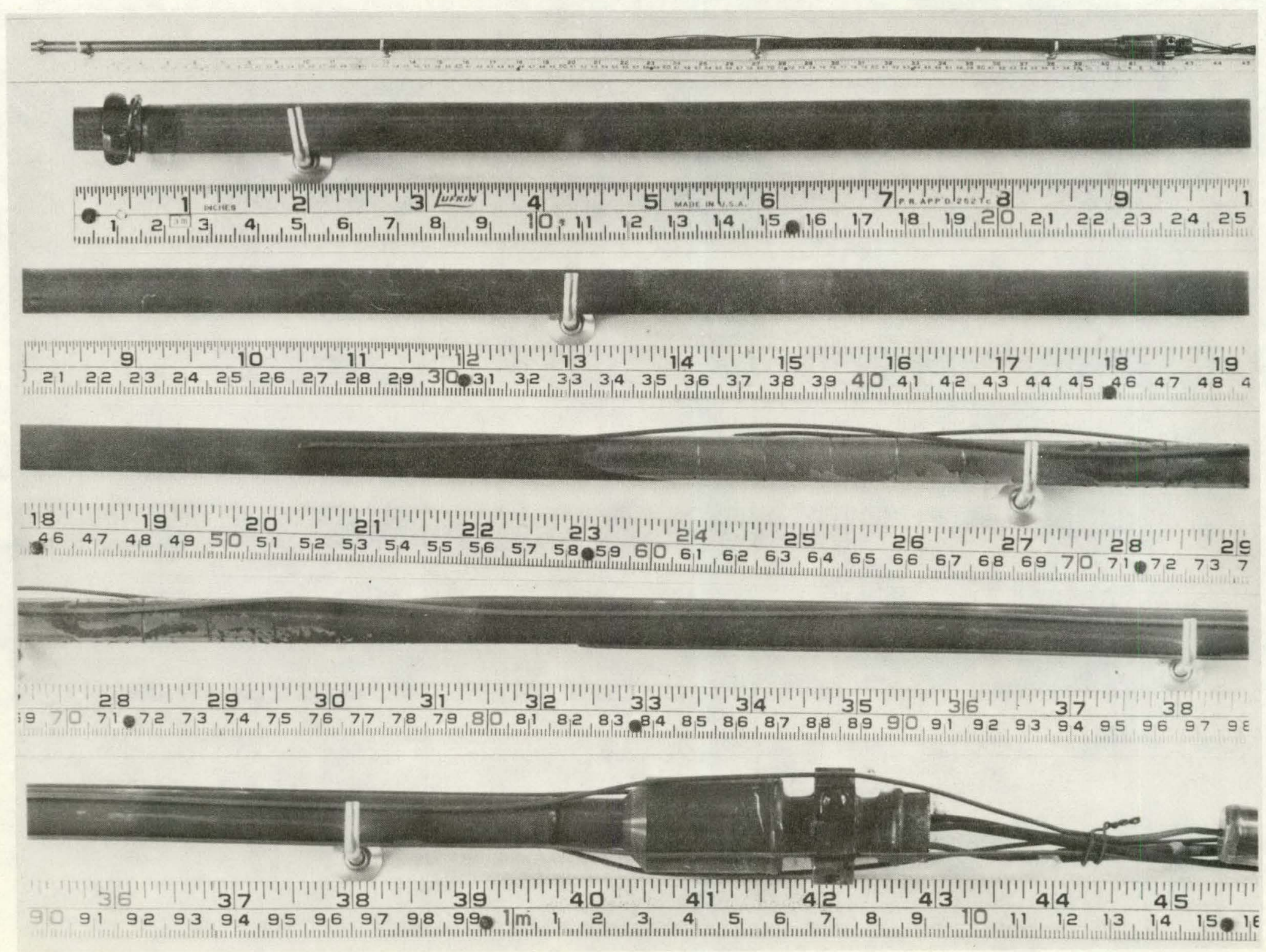

Fig. 31 Test PCM-4, Rod UTA-0016 $\left(0^{\circ}\right)$. 


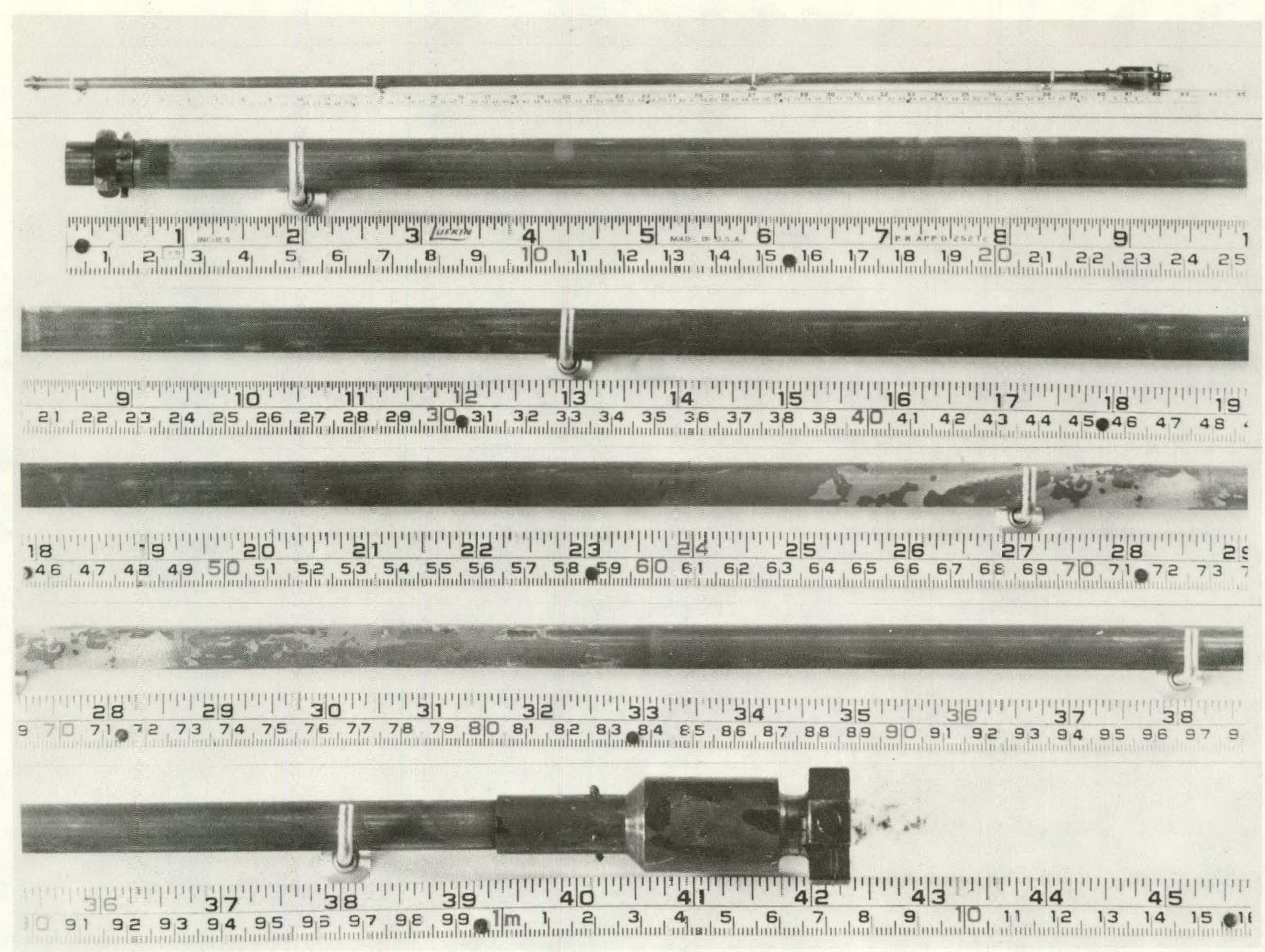

Fig. 32 Test PCM-4, Rod A-0017 $\left(0^{\circ}\right)$. 
Visual examination revealed that all four rods had achieved film boiling during the test as evidenced by cladding waisting at pellet interfaces with $\mathrm{ZrO}_{2}$ spalling within the film boiling zone. Each rod exhibited a black oxide over the remainder of the rod, except for the bottom $0.2 \mathrm{~m}$ of the rod. Within this general region, the black oxide on the four rods is relatively thin and translucent.

The film boiling zone on Rod UTA-0014 extended from 0.61 to $0.84 \mathrm{~m}$, as measured from the bottom of the rod. Cladding waisting at pellet interfaces was apparent over the entire zone. Approximately one-half of the $\mathrm{ZrO}_{2}$ originally within the zone surface had spalled to expose shiny zircaloy base metal. The remaining adherent oxide was characteristic of the black oxide outside the film boiling zone.

The film boiling zone on Rod UTA-0015 extended from 0.56 to $0.82 \mathrm{~m}$, as measured from the bottom of the rod. Near the bottom of the zone $(0.56$ to $0.58 \mathrm{~m})$, virtually all $\mathrm{ZrO}_{2}$ had spalled to expose zircaloy base metal. A uniform thin gray oxide film extended from 0.58 to $0.63 \mathrm{~m}$. From $0.63 \mathrm{~m}$ to the top of the zone, the oxide had a mottled, spotty appearance. Several layers of oxide in various stages of spalling and with appearances ranging from gray to silvery to dull white existed in this region. No black oxide was noted. The cladding waisting at pellet interfaces was most pronounced on this rod. Several large cladding depressions, possibly indicative of chipped pellet corners, were noted at three adjacent pellet interfaces near the bottom of the zone. One particular interface $(0.59 \mathrm{~m})$ contained these depressions around the entire circumference of the rod. Several small, round depressions between interfaces were also noted in this same area.

The film boiling zone on Rod UTA-0016 extended from 0.58 to $0.79 \mathrm{~m}$. Cladding waisting occurred over the entire length of the zone. Most oxide within the zone had spalled. to expose zircaloy base metal. Many small patches of black oxide adhered to the cladding adjacent to thermocouple wires. Several small areas of thin gray oxide were noted near the top of the zone.

The film boiling zone on Rod A-0017 (no cladding thermocouples) extended from 0.61 to $0.84 \mathrm{~m}$. Zircaloy was exposed from 0.61 to $0.66 \mathrm{~m}$. A uniform thin gray oxide was found from 0.66 to $0.71 \mathrm{~m}$. From 0.71 to $0.79 \mathrm{~m}$, multiple mottled layers of oxide in various degrees of spalling were apparent. The remainder of the zone was largely covered by a white oxide. Waisting was observable only at two pellet interfaces near the midpoint of the zone.

All four rods were examined for bowing. Rods UTA-0014 and A-0017 displayed no observable bow. Rod UTA-0015 bowed in the 0 to $180^{\circ}$ plane in the $0^{\circ}$ direction. The cosine-shaped bow extended smoothly from $0.61 \mathrm{~m}$ to the top of the rod, with an estimated maximum deflection of $10 \mathrm{~mm}$ from the 90 to $270^{\circ}$ plane. Rod UTA-0016 displayed a smooth, cosine-shaped bow from 0.46 to $0.71 \mathrm{~m}$ in the 0 to $180^{\circ}$ plane in the $180^{\circ}$ direction. The maximum deflection was estimated to be $5 \mathrm{~mm}$. 
Diametral measurements on the three instrumented rods were made with a micrometer at $50-\mathrm{mm}$ increments outside the film boiling zones and at $12-\mathrm{mm}$ increments inside the film boiling zones. These measurements indicated gradual cladding collapse of 0.20 to $0.23 \mathrm{~mm}$ in the film boiling zones.

1.1.4 Comparison of Calculated and Measured Steady State Test Results. FRAP-T2[a] calculations of the cladding surface temperatures, fuel centerline temperature, and cladding elongation were compared with the corresponding measurements taken during the steady state operating portion of the test. These comparisons are shown in Figures 33, 34, and 35.

The calculated cladding surface temperatures for the three instrumented rods were in good agreement with the steady state measurements. The calculated fuel centerline temperatures were about $200 \mathrm{~K}$ higher than those measured al rud puweis aluve $38 \mathrm{~kW} / \mathrm{m}$.

The calculated cladding elongation was much less than those measured at rod powers in excess of $20 \mathrm{~kW} / \mathrm{m}$. This discrepancy has also occurred on all previous PCM tests. The change in slope of the cladding elongation response curve at rod peak powers of $20 \mathrm{~kW} / \mathrm{m}$ is caused by pellet-cladding interaction.

1.1.5 Discussion and Conclusions. The maximum cladding and fuel temperatures reached by the fuel rods will be determined from detailed posttest metallurgical examination. Measured cladding surface temperatures of 930,1100 , and $1005 \mathrm{~K}$ for Rods UTA-0014, UTA-0015, and UTA-0016 are estimated, on the basis of previous test results, to correspond to actual maximum temperatures of about 1100,1350 , and $1200 \mathrm{~K}$, respectively. Therefore, the actual cladding temperatures at the thermocouple locations on Rods UTA-0014 and UTA-0016 are likely to correspond to the desired maximum cladding temperature range sought ( 810 to $1255 \mathrm{~K}$ ). However, the cladding temperatures may have been higher at elevations on these fuel rods where no instruments were installed. The maximum temperature determined for Rod A-0017, which was not instrumented, is also likely to be in the desired range. Since the maximum cladding temperature of Rod UTA-0015 probably exceeded the $\alpha+\beta$ transition upper boundary of $1240 \mathrm{~K}$, the crystalline structure is expected to be beta phase.

\subsection{Irradiation Effects (IE) Test 1, Preliminary Postirradiation Examination Results}

\section{A. S. Mehner}

The postirradiation examination of the four rods (Rod IE-007, IE-008, IE-009, and IE-010) from Test IE-1 has been initiated. The four rods had been irradiated previously in the Saxton Reactor to burnups of up to $15,800 \mathrm{MWd}$. Preliminary results of the visual examination, dimensional characterization, and metallographic examination are presented in this section.

[a] The power calibration FRAP-T2 calculations were done with the MOD-002, Version 006 used in conjuction with MATPRO MOD-006. 


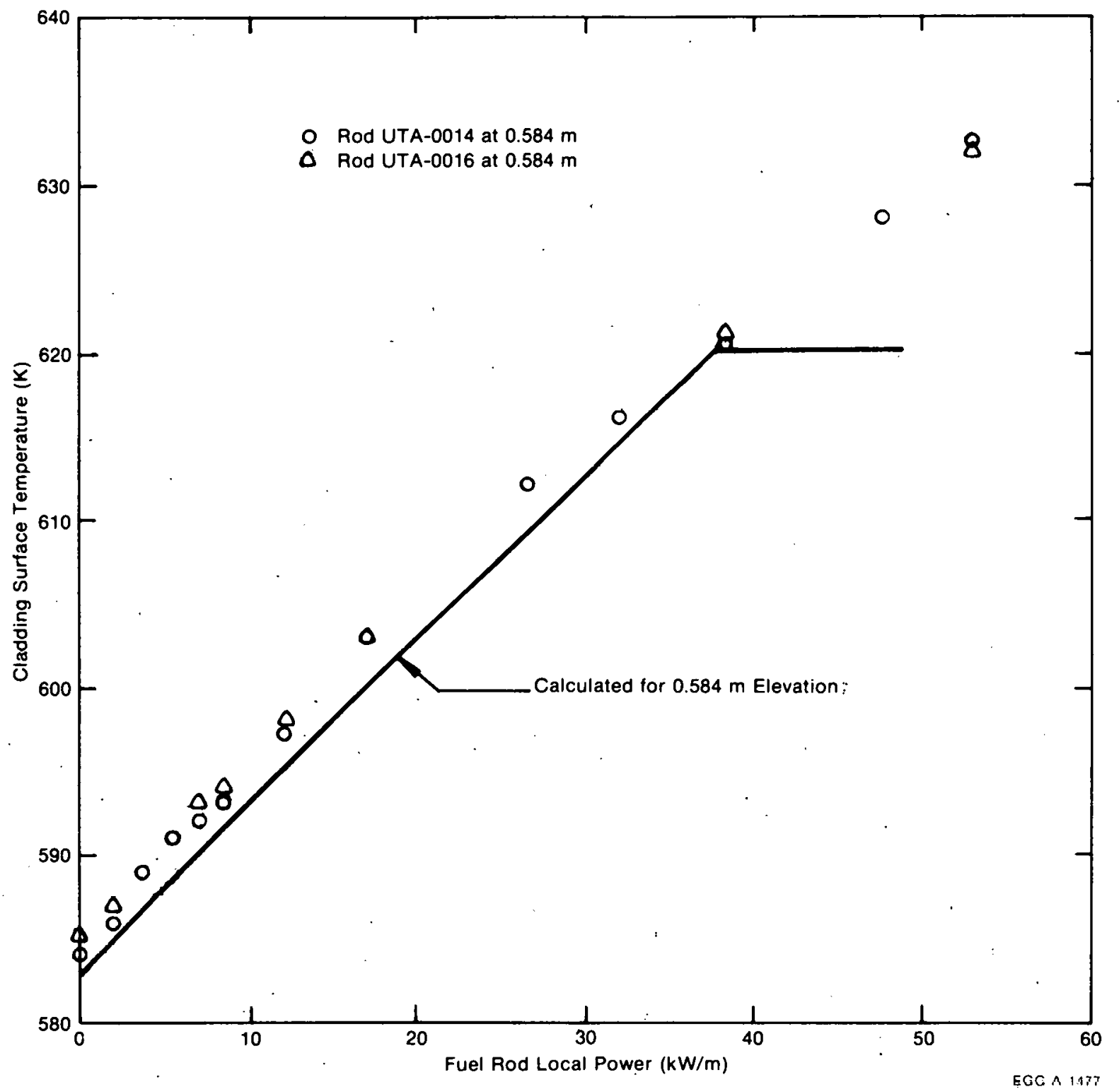

Fig. 33 Predicted and measured cladding surface temperature versus fuel rod local power for steady state phase of Test PCM-4.

1.2.1 Visual Examination. Overall views of the rods are shown in Figure 36. Rod IE-007, which failed during cooldown from film boiling operation, had fractured at the $0.51-\mathrm{m}$ rod elevation. The other three rods were intact, although Rod IE-008 had several cladding splits below the film boiling zone between the 0.25 and $0.37-\mathrm{m}$ rod elevations. No evidence of cladding failure was found on Rods IE-009 and IE-010.

The film boiling zones were characterized by cladding oxidation and evidence of cladding collapse into pellet interfaces and pellet chips. The axial extent of the film boiling zone and the severity of the cladding surface oxidation appeared to correlate with the rod 


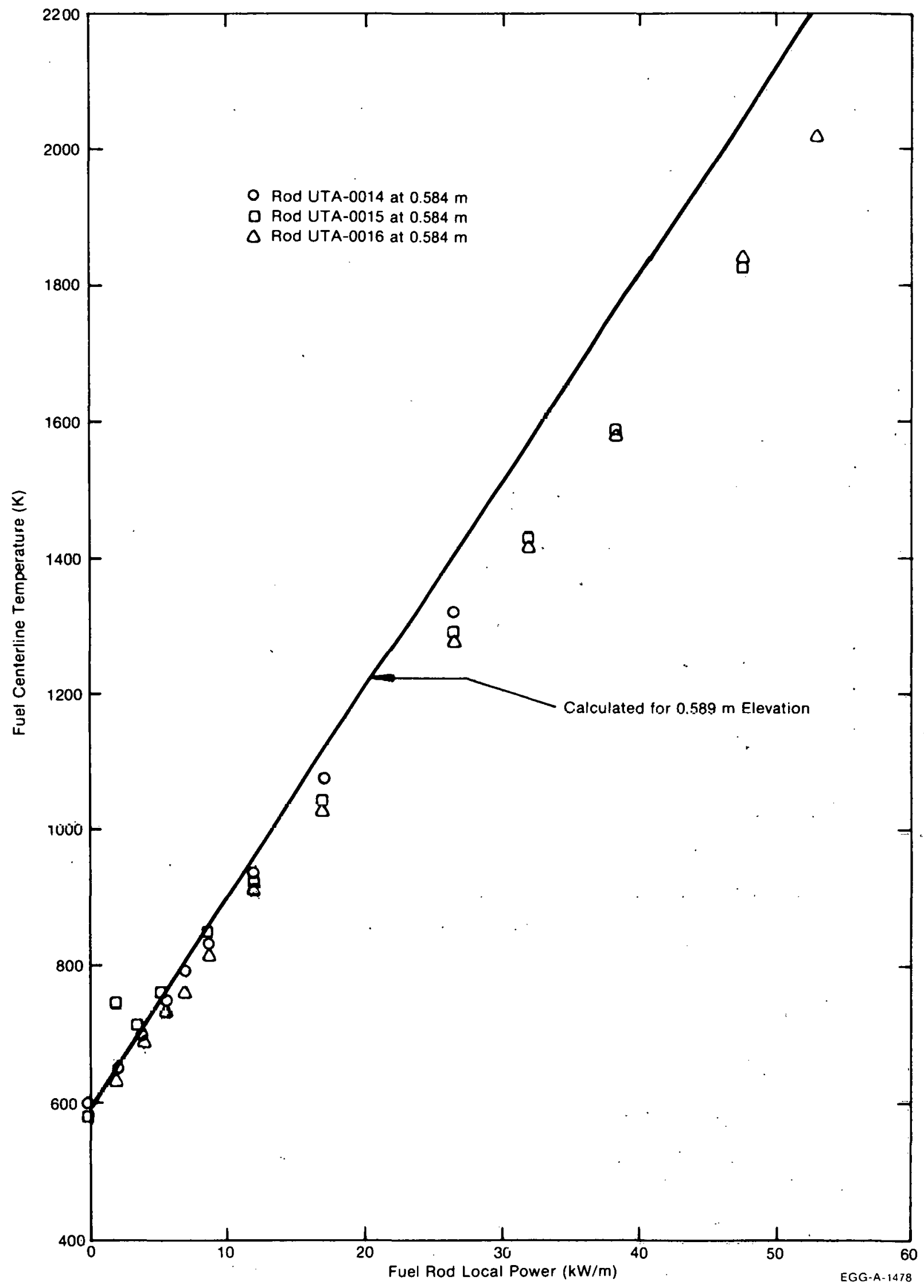

Fig. 34 Predicted and measured fuel centerline temperature versus fuel rod local power for steady state phase of Test PCM-4. 


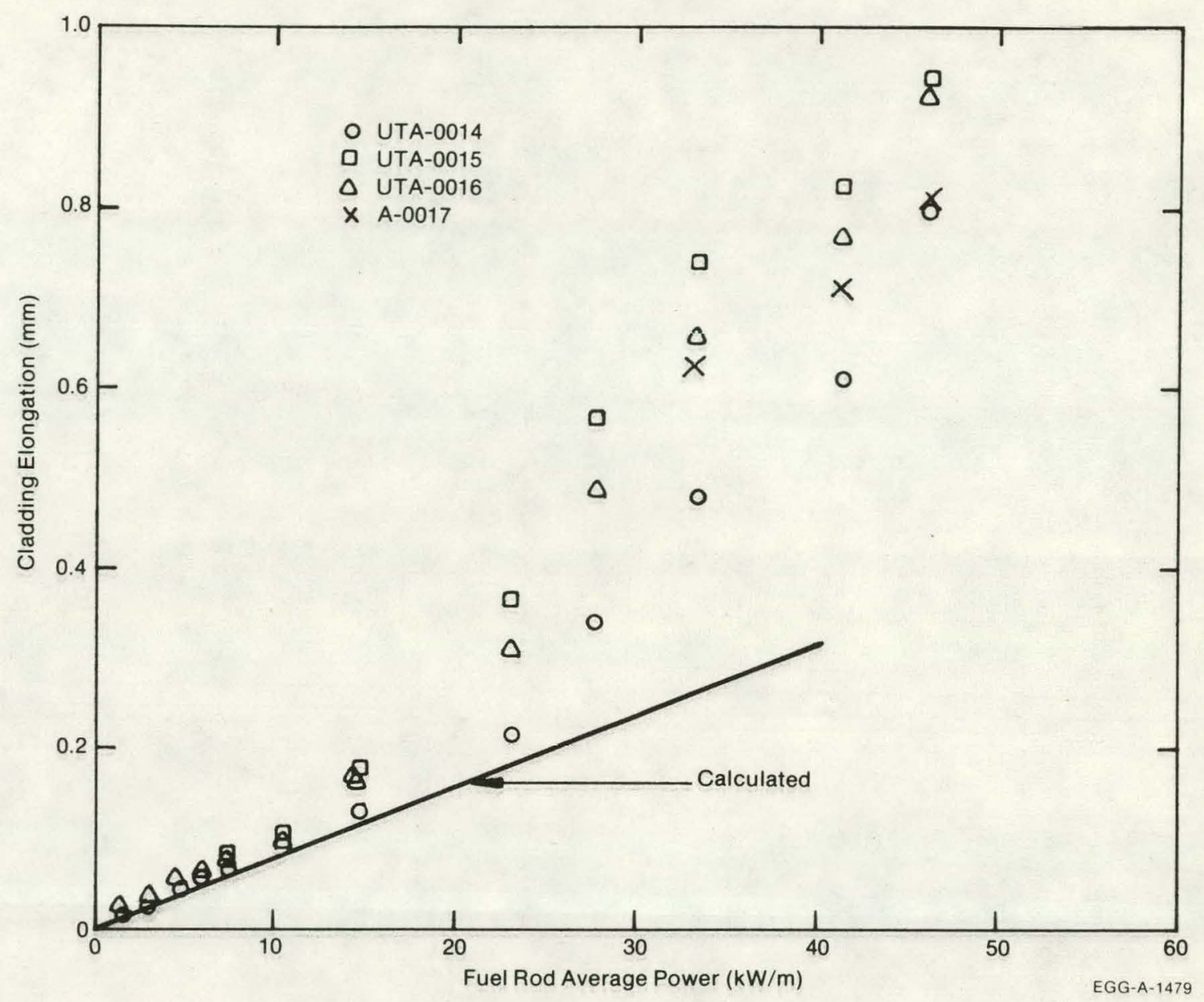

Fig. 35 Predicted and measured cladding elongation versus fuel rod average power for steady state phase of Test PCM-4.

power; i.e., the higher the rod power the larger the film boiling zone and the more severe the surface oxidation (see Table III). The fracture in the cladding on Rod IE-007 appeared extremely brittle. Rod IE-009 was bowed or kinked through the film hniling zone with cladding collapse occurring in regions where fuel had apparently broken up and relocated.

1.2.2 Dimensional Characterization. The diameter of the rods were measured at 0 and $90^{\circ}$ along the length of each rod (see Figure 37). Rods IE-007, IE-008, and IE-010 had increased in diameter in the film boiling zone. This swelling phenomena is contrary to previous experience for rods fabricated with fresh fuel, where the cladding had collapsed onto the fuel column resulting in a decrease in rod diameter. Rod IE-009 did decrease in diameter through the film boiling zone.

1.2.3 Metallographic Examinations and Neutrography. Figures 38 and 39 show neutrographs of, and cross sections from, the film boiling zones of Rods IE-007 and IE-009, respectively. Extensive fuel melting occurred in each rod. Tear-shaped voids had formed in 


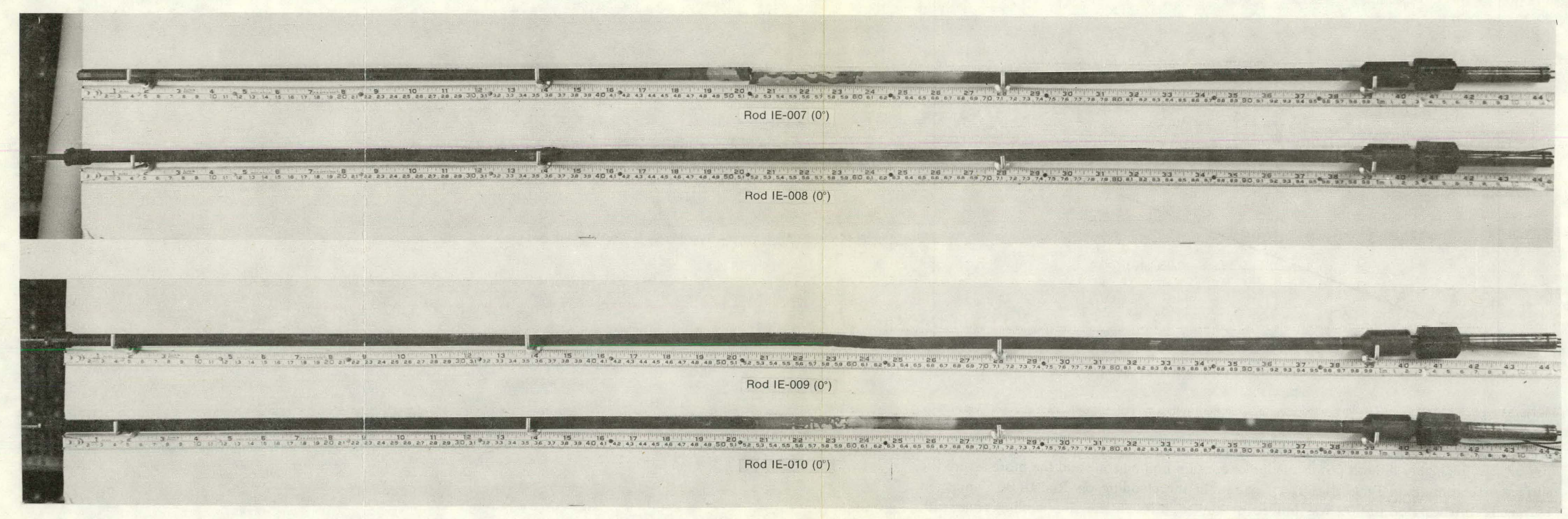

Fig. 36 Posttest view of Rods IE-007, IE-008, IE-009, and IE-010. 
TABLE III

FILM BOILING ZONE ON IE- 1 RODS

\begin{tabular}{|c|c|c|c|}
\hline \multirow{2}{*}{$\begin{array}{l}\text { Rod } \\
\text { Number }\end{array}$} & \multicolumn{2}{|c|}{$\begin{array}{l}\text { Film Boiling } \\
\text { Zone Boundaries } \\
\text { (meters from } \\
\text { bottom of rod) }\end{array}$} & \multirow{2}{*}{$\begin{array}{l}\text { Length of } \\
\text { Film Boiling } \\
\text { Zone (m) } \\
\end{array}$} \\
\hline & Lower & Upper & \\
\hline 00 & 0.445 & 0.685 & 0.240 \\
\hline
\end{tabular}

Observations $^{[\mathrm{a}]}$

Rod failure in film boiling zone; severe cladding oxidation; longest film boiling zone; diameter increase in film boiling zone.

$\begin{array}{llll}\text { IE-008 } & 0.540 & 0.686 & 0.146\end{array}$

Rod intact; cladding cracks below film boiling zone; minor cladding oxidation; short film boiling zone; diameter increase in film boiling zone.

$\begin{array}{llll}\text { IE-009 } & 0.540 & 0.680 & 0.140\end{array}$

Rod intact; shortest film boiling zone; least cladding oxidation; cladding collapse in film boiling zone.

$\begin{array}{llll}\text { IE- } 010 & 0.508 & 0.686 & 0.178\end{array}$

Rod intact; relatively severe oxidation; second longest film boiling zone; diameter increase in film boiling zone. 
Rod E-007

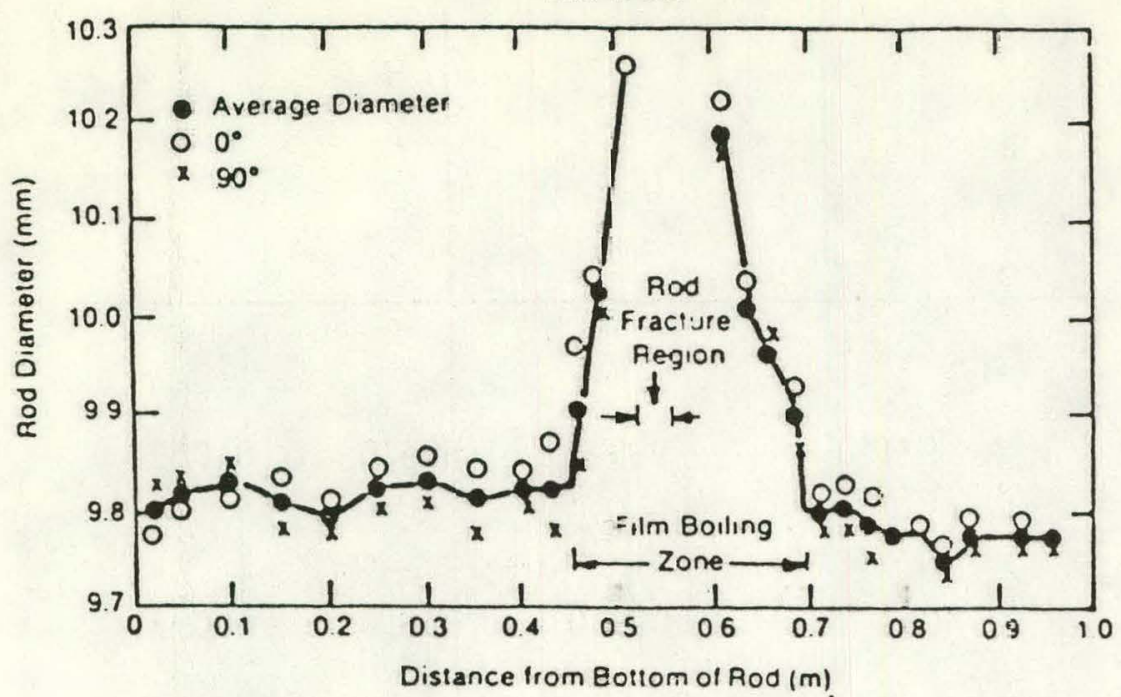

Rod IE-OCO

$+$

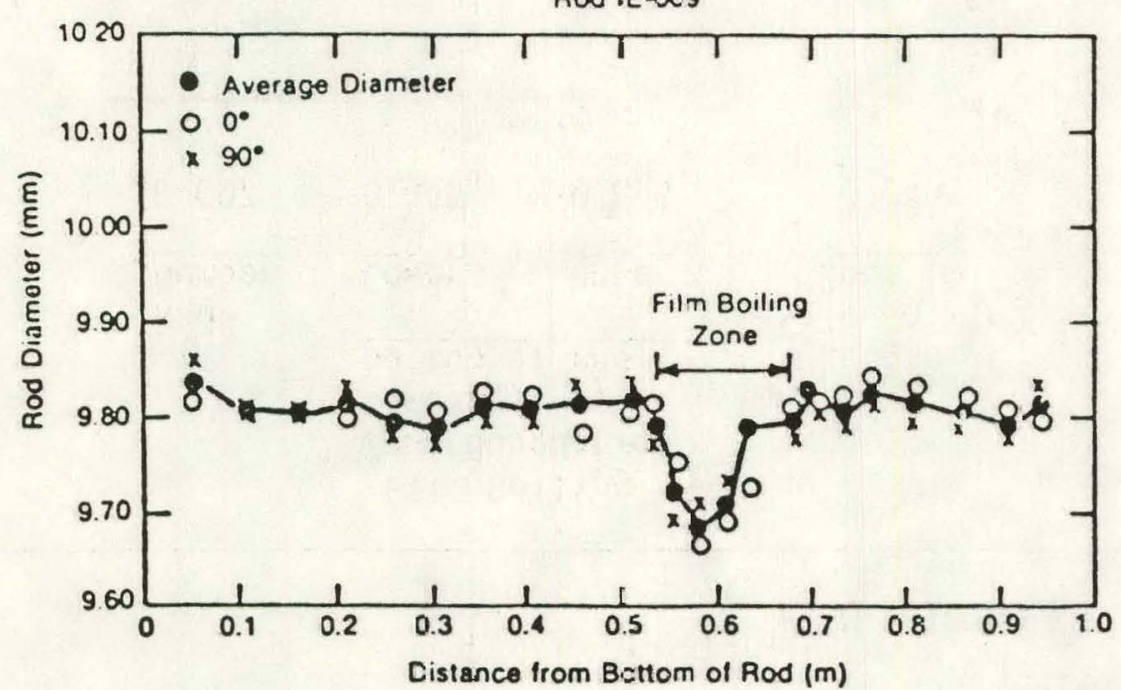

Rod IE-008

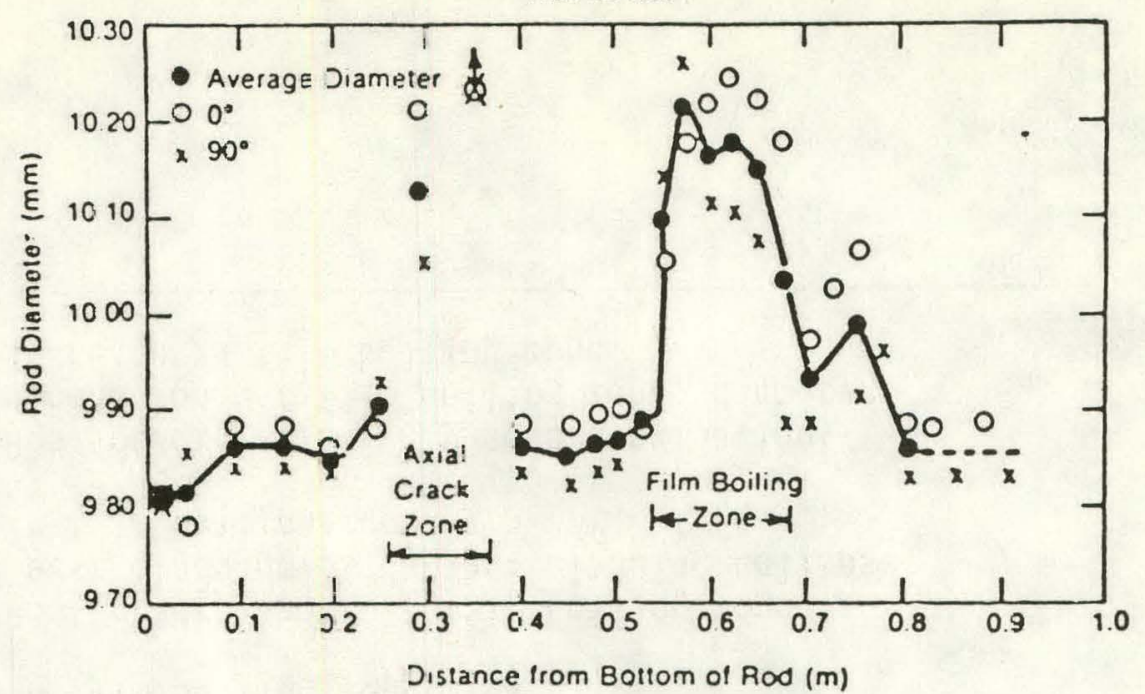

Rod IE -010

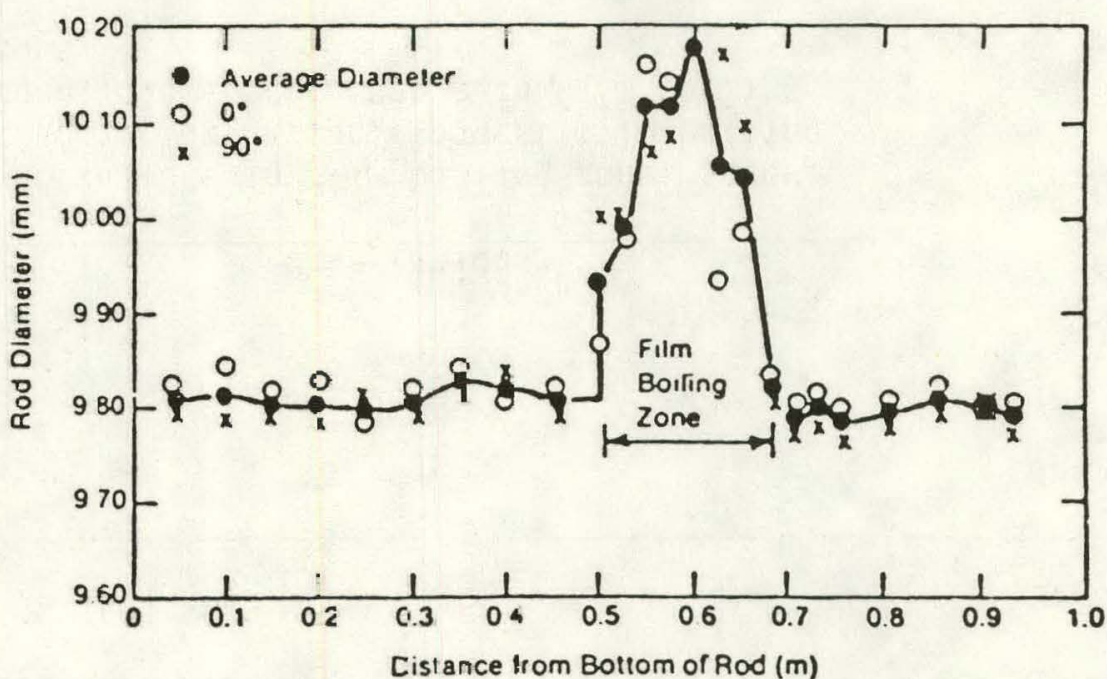

Fig. 37 Posttest diameter masurements on Test IE-1 irradiated rods. 


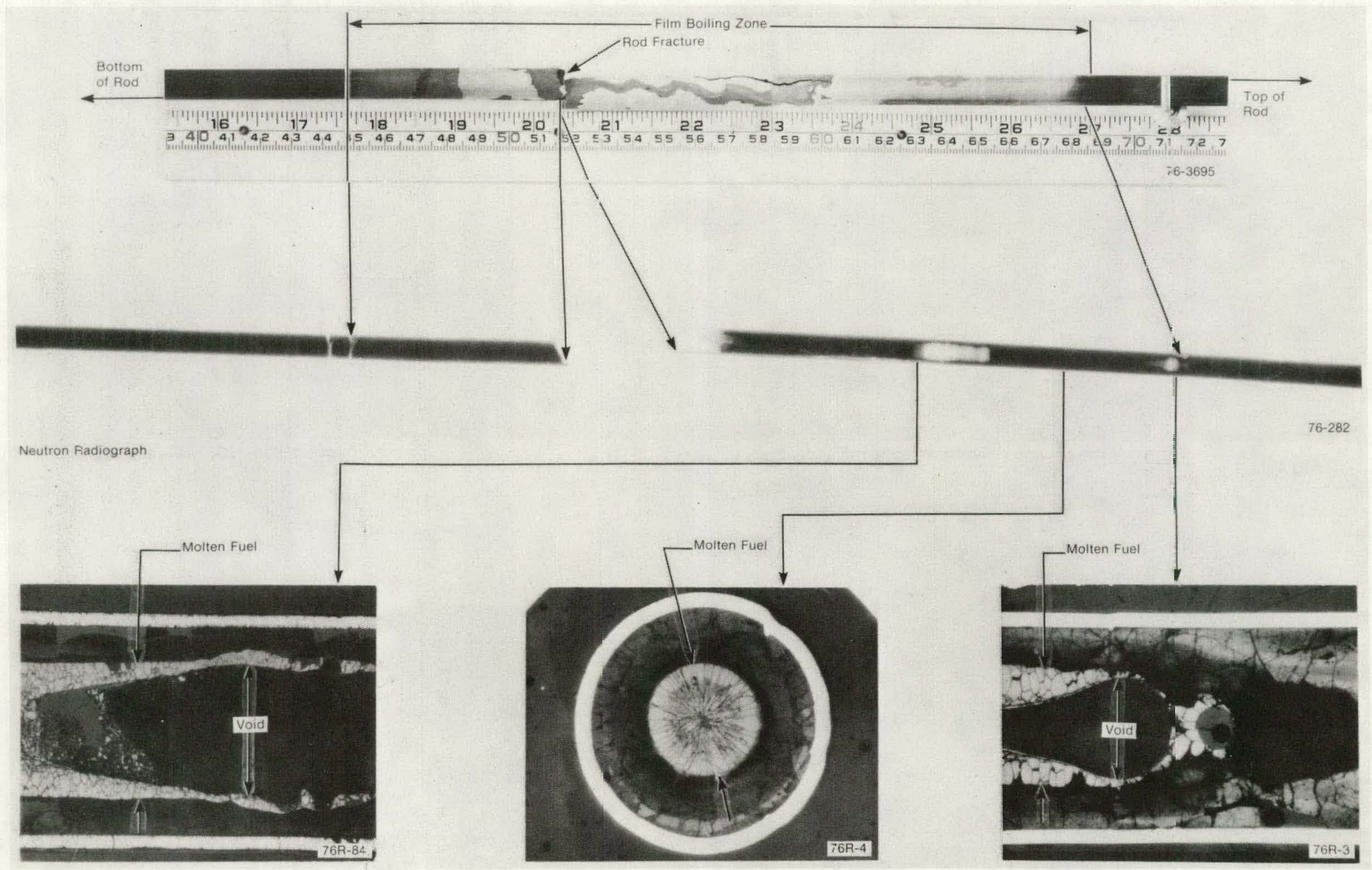

Fig. 38 Film boiling on Rod IE-007, which resulted in heavy surface oxidation, fuel melting, and fuel relocation. 


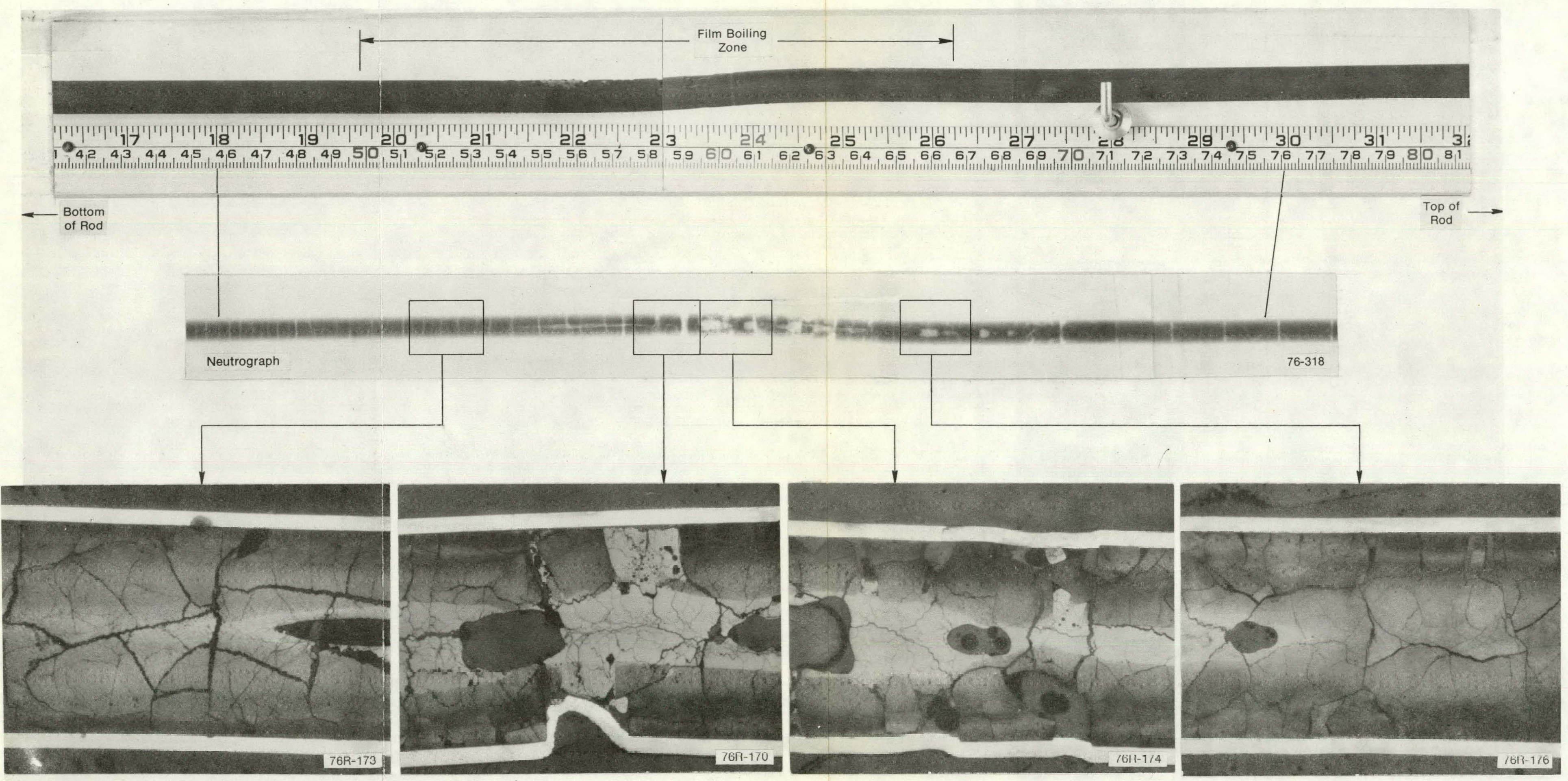

Fig. 39 Metallography from Rod IE-009. 
the center of the fuel of Rod IE-007 due to molten fuel slumping. Severe cladding oxidation had occurred in the film boiling zone, with the cladding wall at some locations nearly reacted to oxygen-stabilized alpha-zirconium $(\alpha-\mathrm{Zr})$ as shown in Figure 40 . The inside $\alpha-\mathrm{Zr}$ layer due to the fuel cladding reaction was essentially the same thickness as the outside $\alpha-\mathrm{Zr}$ layer formed by the cladding-water reaction. At the location shown in Figure 40, the cladding temperature was estimated to be $1800 \mathrm{~K}^{[\mathrm{a}]}$.

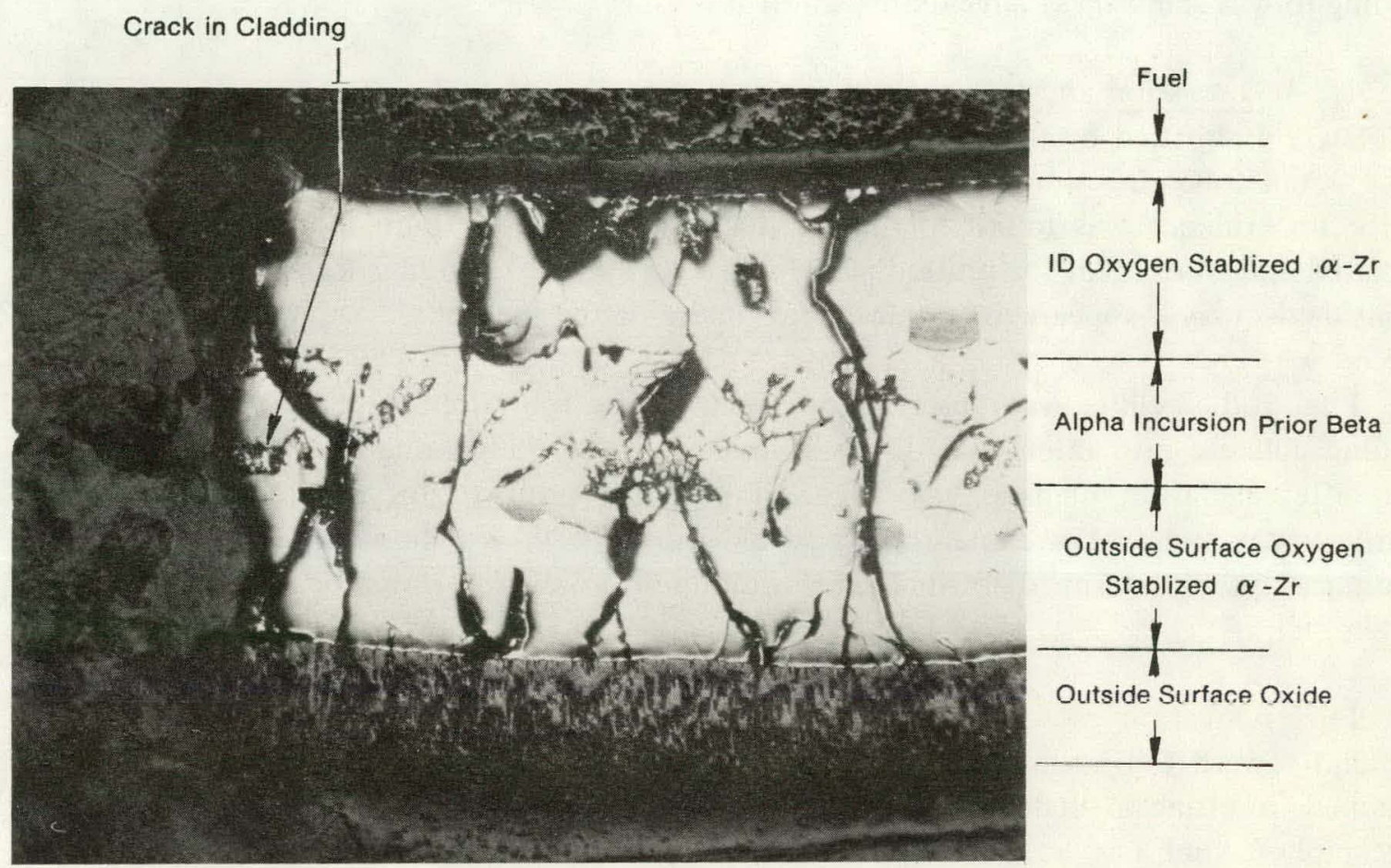

Fig. 40 Highly embrittled cladding from Rod IE-007.

[a] Cladding temperature estimated was calculated using Pawel's expression relating $\mathrm{Zr}$-water reaction layer thickness, time at temperature, and temperature by the method outlined in Reference 14. 
In Rod IE-009, molten fuel appeared to have been extruded from the center of the fuel pellets outward toward the cladding through regions in the pellet where the fuel pellet had cracked and relocated. In isolated areas, the molten fuel had definitely contacted the cladding; however, only a relatively minor fuel-cladding reaction occurred (see Figure 41). In most regions, the molten fuel came very close to the cladding but did not appear to have impinged on the cladding or, if it did touch the cladding, the fuel-cladding reaction was very minor.

On Rod IE-009, the cladding was subjected to severe deformation strains. Prior irradiation damage must have been annealed out before the cladding collapse for the cladding to withstand these large deformation strains.

1.2.4 Discussion and Conclusions. Even though Rod IF-007 cladding was essentially completely embrittled by the $\mathrm{Zr}$-water and $\mathrm{Zr}-\mathrm{UO}_{2}$ reactions, rod failure did not oscur until about 3 min following shutdown from film boiling operation. Based on these preliminary results, no evidence was found to suggest that a more severe failure mode exists for the irradiated fuel rods than for unirradiated fuel rods exposed to similar transients, although the inside $\alpha$-Zr layer appears to be thicker for the irradiated fuel rods.

Fuel rod swelling was observed on three of the four irradiated rods. Evidence of cladding collapse into interpellet dishes suggests that the cladding collapsed onto the fuel stack after radiation damage had annealed out and prior to the substantial cladding embrittlement and fuel swelling. Two possible causes of the swelling are (a) expansion of fission gas bubbles trapped at fuel grain boundaries and (b) volumetric increase of the molten fuel.

Fuel relocation as found in Rod IE-009 provides pathways for molten fuel to be extruded toward the inside surface of the cladding. The molten fuel is apparently rapidly quenched, resulting in little or no molten fuel-cladding reaction. For conditions tested to date, molten fuel has been contained within the fuel rod for both unirradiated and irradiated fuel rods, even when the molten fuel radius was a substantial fraction of the pellet radius or a pathway existed for molten fuel to be extruded to the cladding.

\subsection{Irradiation Effects Test 2, Preliminary Results}

A. S. Mehner, C. M. Allison, L. C. Farrar

Test IE-2, the fourth test in the irradiation effects test series, was conducted October 7, 8, and 9, 19/6. 'The test was performed with four PWR rods fabricated with fresh fuel. Two rods (Rods IE-011 and IE-012) were fabricated with cladding previously irradiated in the Saxton Reactor, and two rods (Rods IE-013 and IE-014) were fabricated with unirradiated cladding.

The objectives of the test were to provide data on fuel rods with variation in gap size and cladding irradiation damage including: 

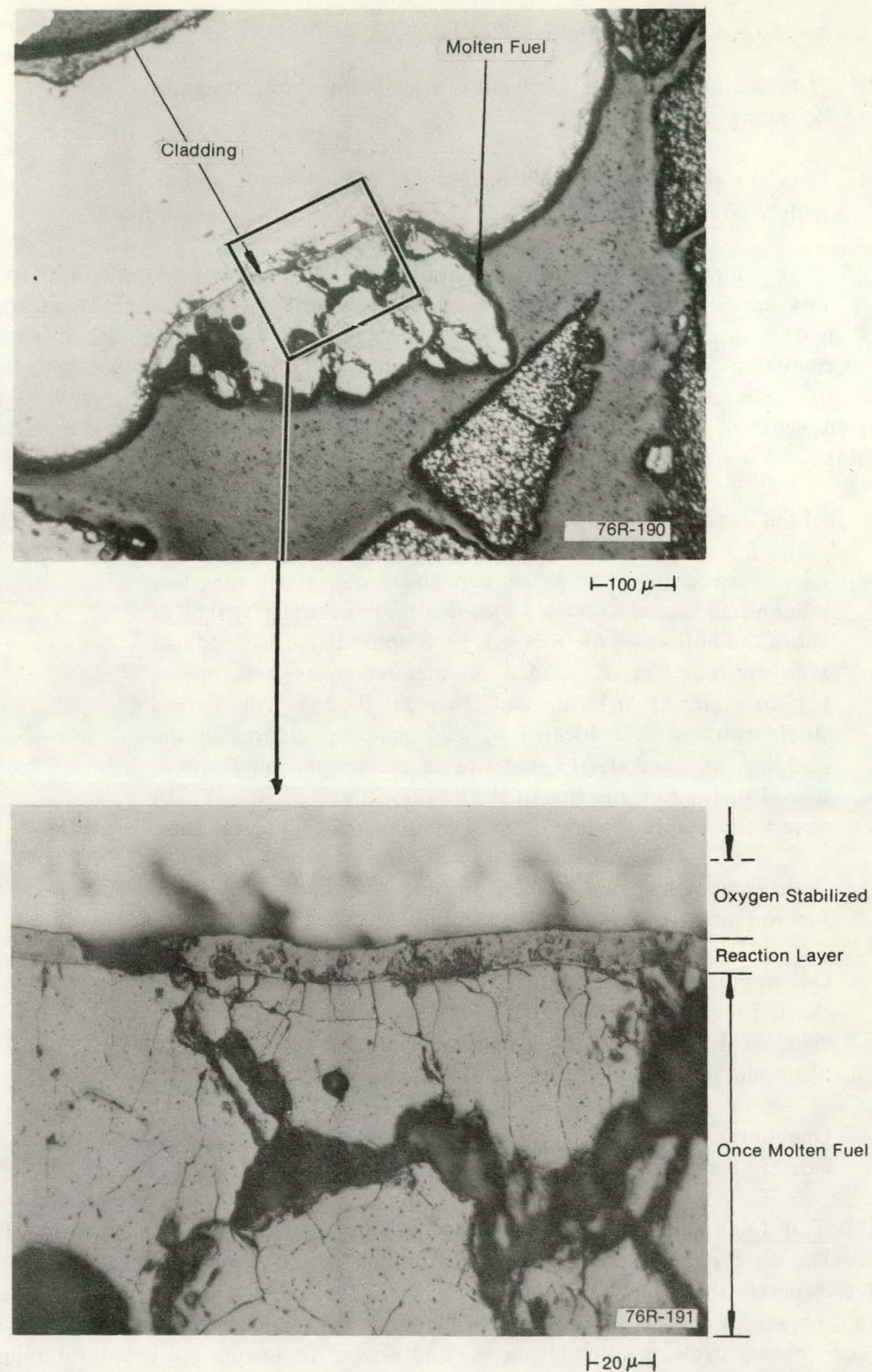

Fig. 41 Isolated area on Rud IE-009 where cladding had collapsed into region of fuel relocation and fuel had impinged on the inside surface of the cladding. 
(1) Steady state data at various fuel rod power levels

(2) Data on fuel cladding mechanical interaction during transient operating conditions

(3) Data for post-CHF fuel rod behavior at high power (approximately $66 \mathrm{~kW} / \mathrm{m}$ peak).

1.3.1 Test Configuration and Instrumentation. Test IE-2 was performed with the four fuel rods positioned symmetrically in the in-pile test space. Each fuel rod was contained in its own coolant shroud, which was instrumented for flow rate and fuel rod power determination. The system was designed to allow all four fuel rods to experience essentially the same reactor power and coolant flow changes. In addition, seven cobalt self-powered neutron detectors (SPNDs) were located in the test train to measure axial neutron flux.

The fuel rod instrumentation consisted of the following:

(1) Each irradiated fuel rod had two zircaloy-sheathed tungstenrhenium alloy, spring-loaded cladding thermocouples at $0.62 \mathrm{~m}$ above the bottom of the fuel rod. Each unirradiated fuel rod had attached four Type S, spaded tip thermocouples, with one at $0.52 \mathrm{~m}$, one at $0.72 \mathrm{~m}$, and two at $0.62 \mathrm{~m}$. All Type S thermocouples were located in 0.25 -mm-deep grooves in the cladding. On Rod IE-013, the tips of the thermocouples were brazed to the rod; the tips of the thermocouples on Rod IE-014 were welded to the rnd, with braze filler matcrial filling the gaps.

(2) Each plenum had one 17-MPa pressure transducer to measure fuel rod internal pressure in the plenum.

(3) One tungsten-rhenium centerline thermocouple was located at the $0.74-\mathrm{m}$ elevation on each of the two rods with irradiated cladding. Each of the two rods with unirradiated cladding had an ultrasonic thermometer centered at the $0.62-\mathrm{m}$ elevation.

(4) Oıe linear variable differential transformer (LVDT) was provided to measure changes in the axial length of each fuel rod.

1.3.2 Test Conduct. The nominal operating conditions for Test IE-2 are shown in Figure 42. The first part of the test (preconditioning period) consisted of (a) a series designed to separate the effect of inlet temperature, flow rate, and reactor power on the relationship between fuel rod power and SPND current, (b) two cycles of gap conductance testing, and (c) Iwo cycles of slowly varying rod power. Following the preconditioning period, the average fuel rod peak linear heat rating of all four rods was increased from 23 to $64 \mathrm{~kW} / \mathrm{m}$ at a ramp rate of $3 \mathrm{~kW} / \mathrm{m}$-min. After an hour at $64 \mathrm{~kW} / \mathrm{m}$, the flow was reduced at constant power to induce film boiling on the fuel rods. 

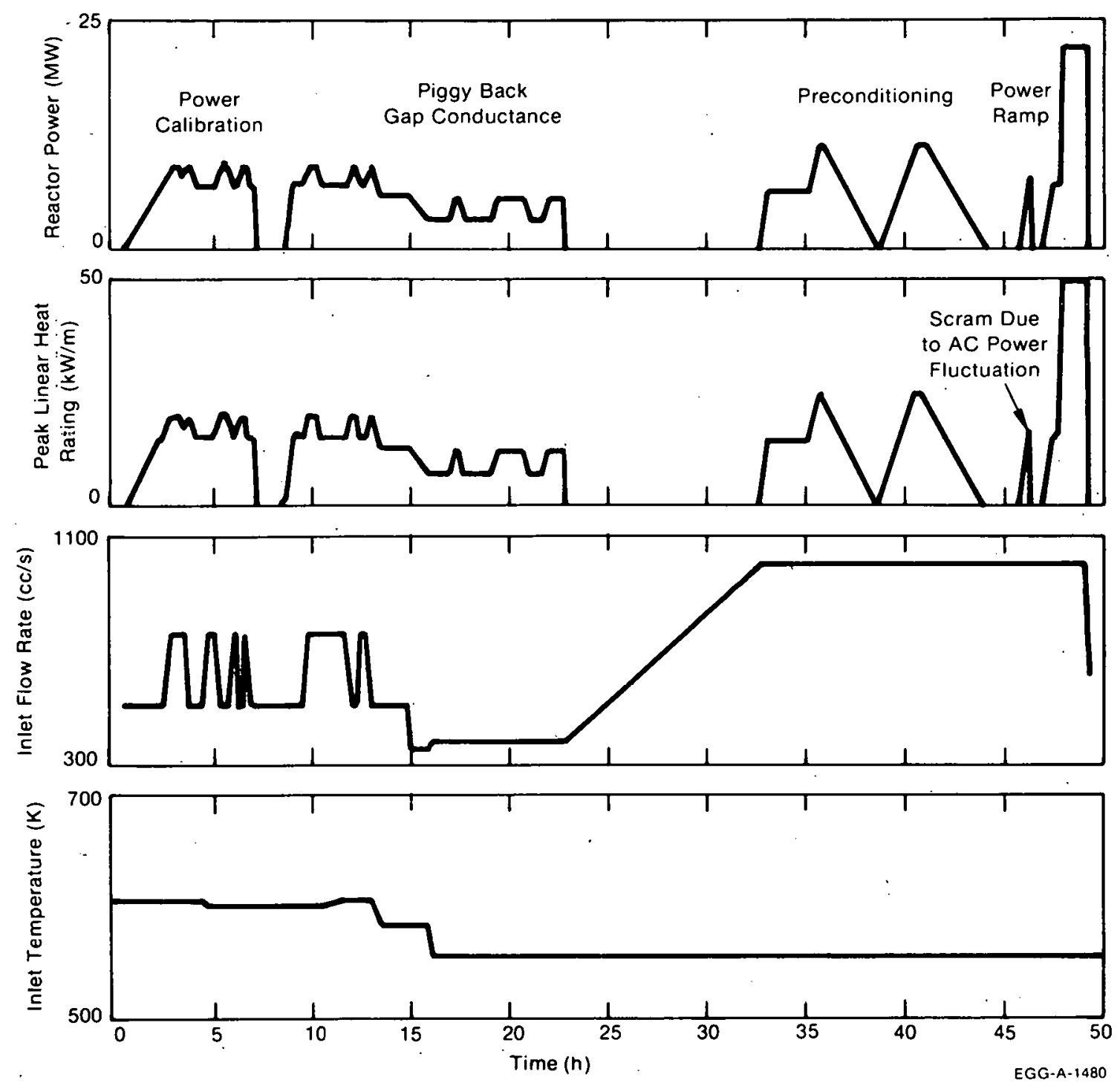

Fig. 42 Nominal operating conditions during Test IE-2. .

The first series of power calibration cycles consisted of 16 steps at all possible combinations of two levels of flow rate $\left(505\right.$ and $\left.757 \mathrm{~cm}^{3} / \mathrm{s}\right)$, inlet temperature $(583$ and $695 \mathrm{~K})$, and average fuel rod peak linear heat rating $(23$ and $33 \mathrm{~kW} / \mathrm{m})$. The combination of flow rate, inlet temperature, and rod linear heat rating was chosen using a $2^{3}$ factorial design with one complete replicate to provide estimates of variances. The steps were randomly arranged in time, with a constraint on the inlet temperature as shown in Figure 42. The design was also constrained so the effect of time, electronic drift, etc., was confounded with the three-factor interaction.

A relationship was developed so rod power could be calculated from SPND current in the event of instrument failure or conditions where a thermal-hydraulic balance was impossible (i.e., the flow reduction portion of the test where the coolant outlet 
temperatures are at saturation). Peak linear heat ratings versus SPND current are shown in Figures 43 through 46 . Ratios of linear heat ratings to average heat ratings for Rods IE-011, IE-012, IE-013, and IE-014 were $1.00,1.00,1.02$, and 0.98; respectively.

Following the preconditioning period, the reactor power was increased at a ramp rate of $3 \mathrm{~kW} / \mathrm{m}$-min to provide a fuel rod power of $64 \mathrm{~kW} / \mathrm{m}$. Once steady state was obtained at this power level, a calculation of fuel rod power, using coolant flow and temperature differential, indicated that the fuel rods had powers of $65.3,64.8,62.2$, and $61.4 \mathrm{~kW} / \mathrm{m}$ for Rods IE-011, IE-012, IE-013, and IE-014, respectively. The fuel rod power, coolant inlet temperature, and coolant flow were held constant for approximately $1 \mathrm{~h}$.

After about $1 \mathrm{~h}$ at $64 \mathrm{~kW} / \mathrm{m}$, the flow reduction portion of the test was initiated. The fuel rod coolant flow rate, nominally the same for all four rods, was reduced from $1000 \mathrm{~cm}^{3} / \mathrm{s}\left(240 \mathrm{~kg} / \mathrm{s} \cdot \mathrm{m}^{2}\right)$. Each flow reduction step was held constant for approximately $1 \mathrm{~min}$. At a flow of approximately $530 \mathrm{~cm}^{3} / \mathrm{s} 2600 \mathrm{~kg} / \mathrm{s}^{2} \mathrm{~m}^{2}$ ), the LVDT on Rod IE-011 indicated the onset of film boiling (the onset of film boiling was calculated as $560 \mathrm{~cm}^{3} / \mathrm{s}$ ). At this point, the flow was reduced to $460 \mathrm{~cm}^{3} / \mathrm{s}\left(2390 \mathrm{~kg} / \mathrm{s} \cdot \mathrm{m}^{2}\right)$. During this flow reduction, film boiling was indicated on the remaining fuel rods. Table IV provides the magnitudes of the measurements recorded on the strip charts for all four fuel rods during the film boiling portion of the experiment. After the flow reduction to $460 \mathrm{~cm}^{3} / \mathrm{s}$, the flow was held constant for about $1 \mathrm{~min}$, after which the reäctor was shut down.

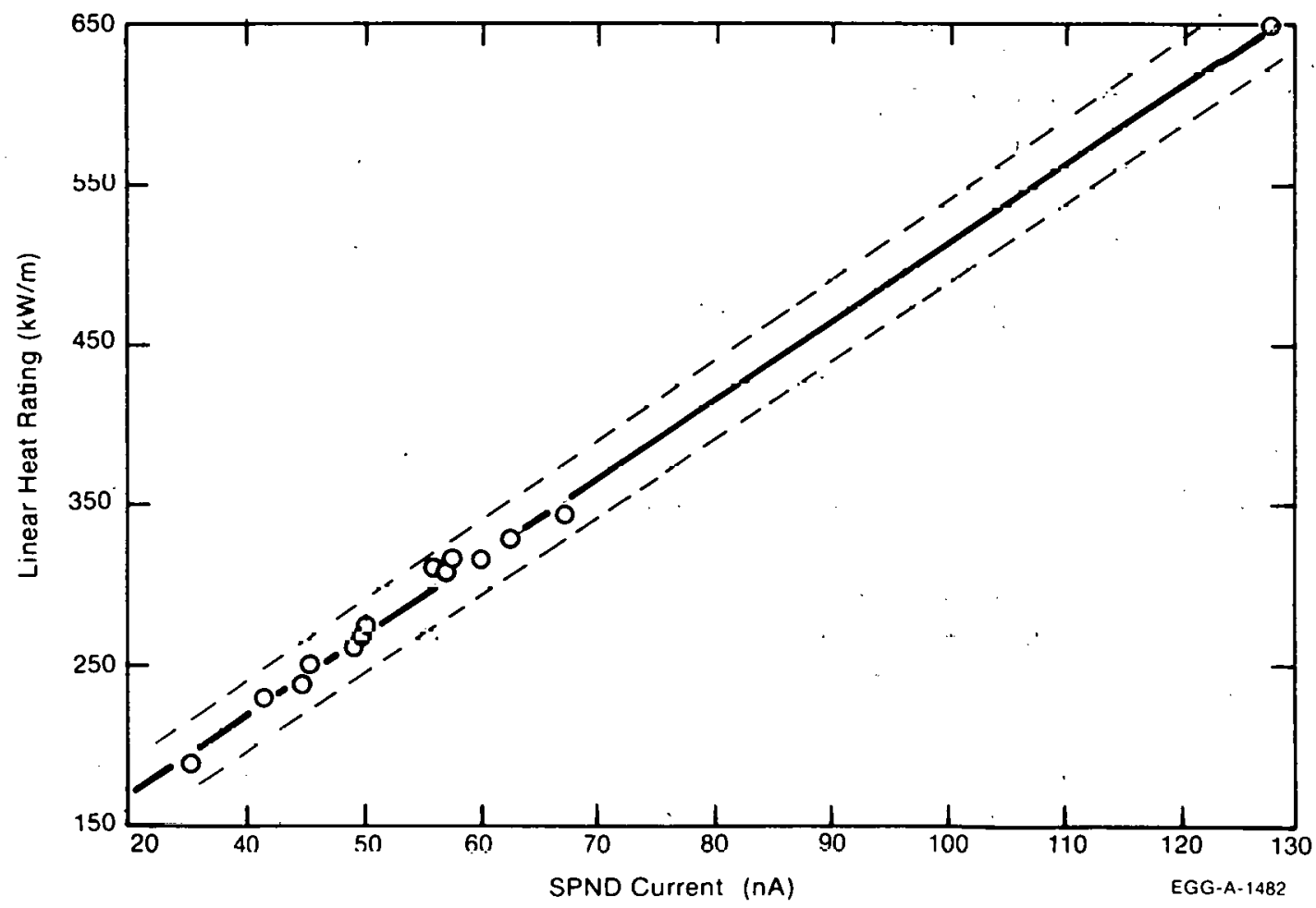

Fig. 43 Fuel rod power for Rod IE-011 versus SPND current for Test IE-2. 


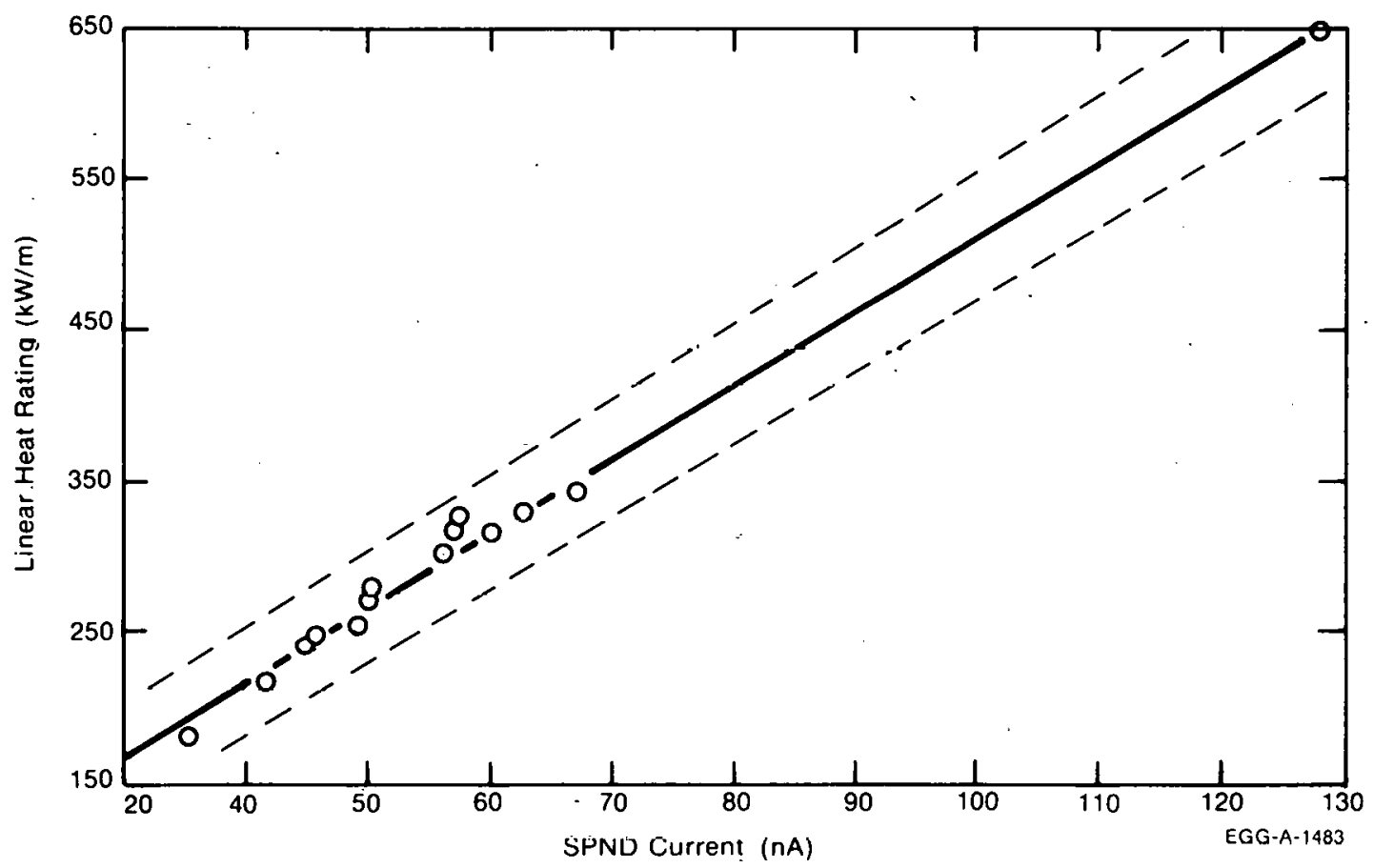

Fig. 44 Fuel rod power for Rod IE-2 versus SPND current for Test IE-2.

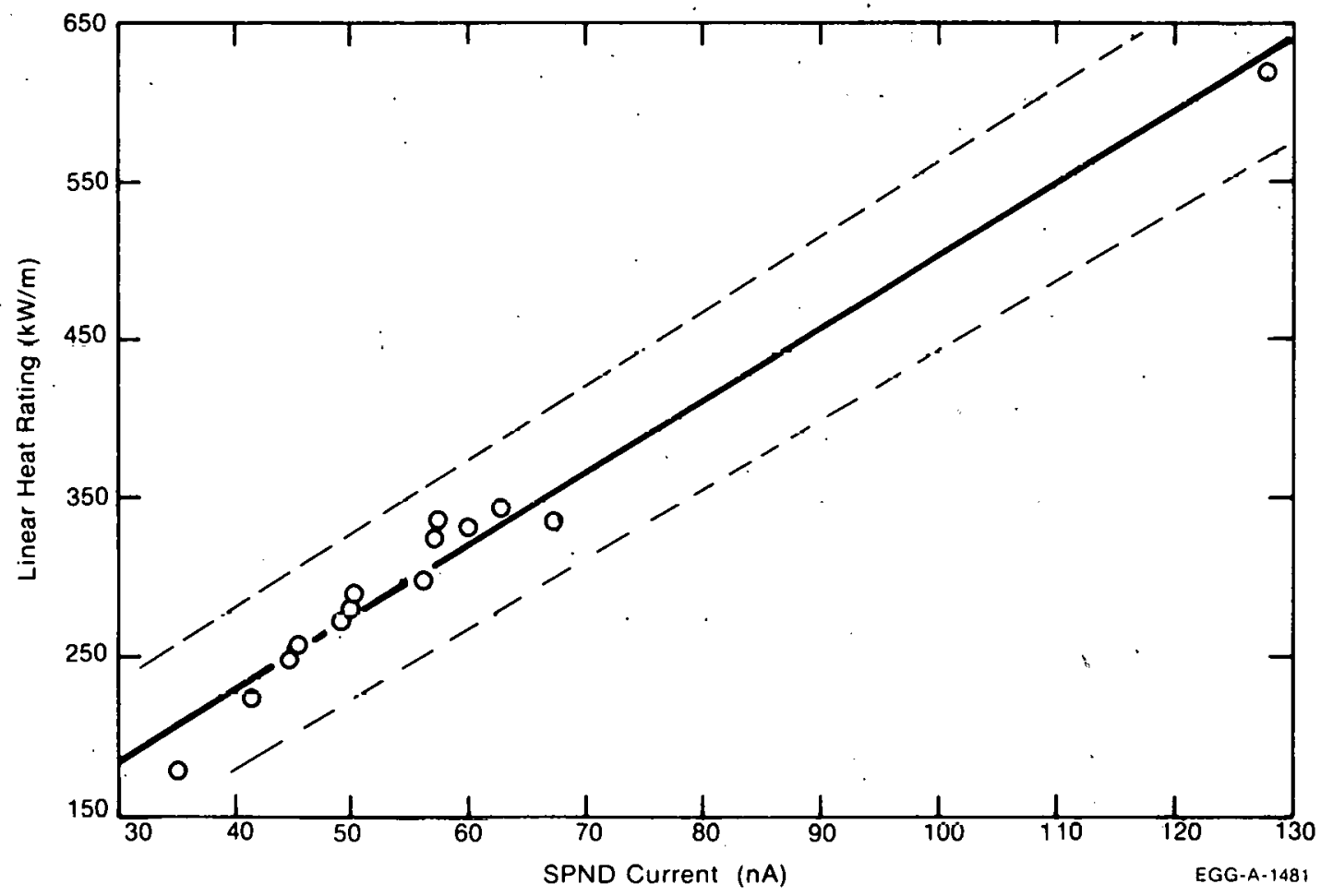

Fig. 45 Fuel rud jowei fui Rod IE-013 versus SPND current for Tost IE-2. 


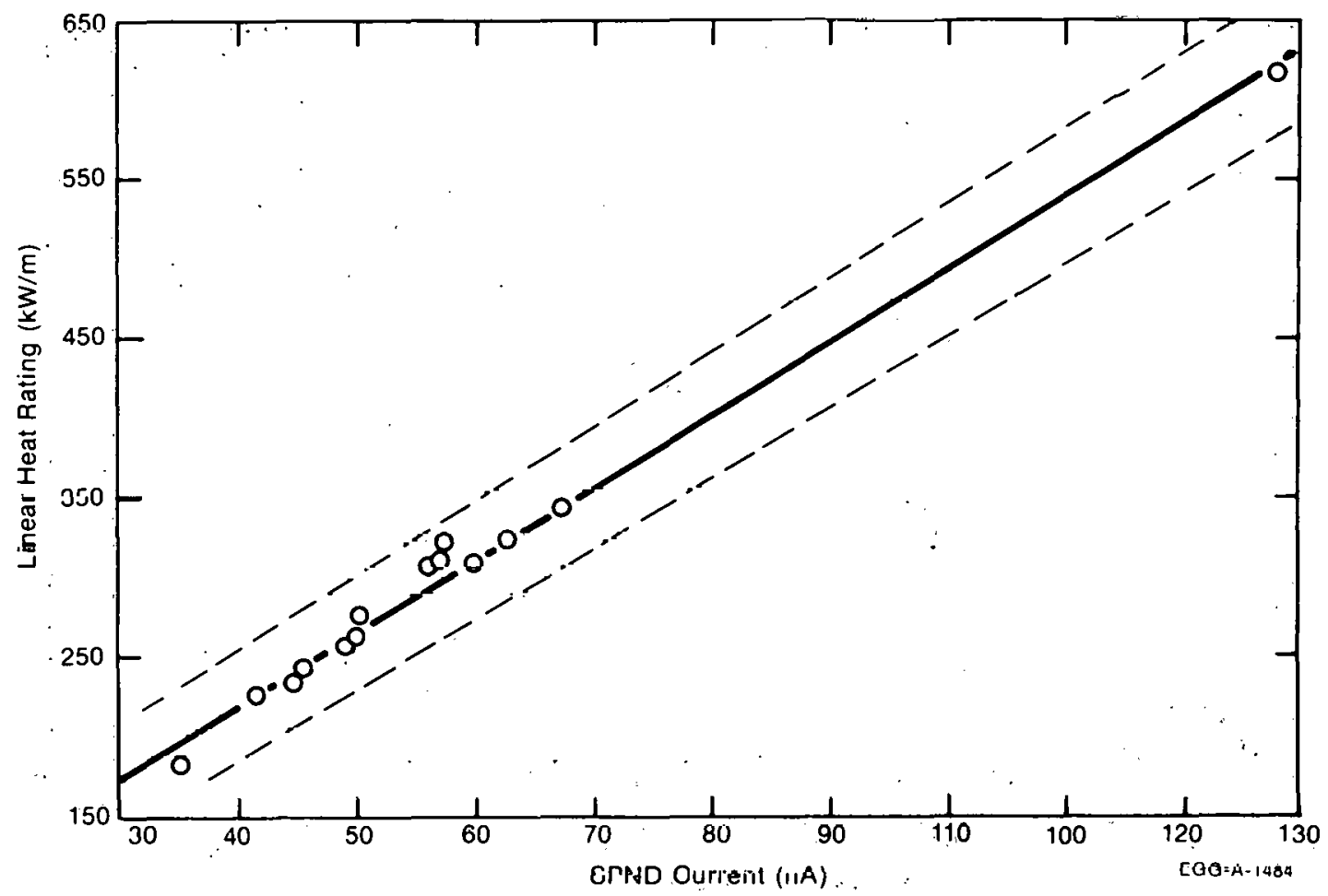

Fig. 46 Fuel rod power for Rod IE-014 versus SPND current for Test IE-2.

Figures 47,48 , and 49 provide a history for various measurements made on Rods IE-011, IE-013, and IE-014 during the flow reduction. Data for Rod IE-012 were not recorded on strip charts.

Fuel rod pressure readings and the loop fission monitor indicated that none of the fuel rods failed during or after the film boiling portion of the test.

1.3.3 Discussion and Conclusions. The test met most objectives of providing data on fuel rods with designed differences in gap and cladding irradiation damage. Film boiling was established on all rods, with film boiling being established near the calculated flow rate of $560 \mathrm{~cm}^{3} / \mathrm{s}\left(2750 \mathrm{~kg} / \mathrm{s} \cdot \mathrm{m}^{2}\right)$ at a rod peak power of $66 \mathrm{~kW} / \mathrm{m}$. The fuel rods in this experiment experienced film boiling at a flow of about $530 \mathrm{~cm} / \mathrm{s}\left(2600 \mathrm{~kg} / \mathrm{s} \cdot \mathrm{m}^{2}\right)$ and a rod peak power of $64 \mathrm{~kW} / \mathrm{m}$. The predicted peak cladding temperature at the $0.61-\mathrm{m}$ elevation was $1100 \mathrm{~K}$ during film boiling; measured cladding temperatures at the $0.62-\mathrm{m}$ clevation were $1080 \mathrm{~K}$ (Rod IE-013) and $1020 \mathrm{~K}$ (Rod IE-014). Figure 50 shows the comparison between calculated fuel centerline temperatures and some measured values.

Most of the critical measurements were completed successfully. Of the critical instruments that failed, the LVDT failures compromised the test objectives most, because the pellet-cladding interaction data prior to film boiling and the thermally induced axial strain during film boiling were not obtained. The LVDT on Rod IE-013 failed at the beginning of the test, and the LVDTs on Rods IE-014 and IE-012 were suspect. The 


\section{TABLE IV}

\section{MEASUREMENT SUMMARY MADE OF FUEL RODS DURING FILM BOILING}

\begin{tabular}{|c|c|c|c|c|c|c|c|c|c|c|c|}
\hline \multirow[b]{2}{*}{ Fuel Rod } & \multirow[b]{2}{*}{$\begin{array}{c}\text { Pretest Fuel } \\
\text { Roc Internal } \\
\text { Pressure at } 600 \\
(\mathrm{MPa}) \\
\end{array}$} & \multirow{2}{*}{$\begin{array}{l}\text { Maxinum } \\
\text { Interna } \\
\text { Absolute } \\
\text { Reading } \\
\text { (MPa) }\end{array}$} & \multirow{2}{*}{$\begin{array}{l}\text { Fuel Rod } \\
\text { Pressure } \\
\text { Relative } \\
\text { Increase } \\
\text { (MPa) }\end{array}$} & \multicolumn{2}{|c|}{$\begin{array}{c}\text { Maximum Cladding } \\
\text { Elongation }\end{array}$} & \multicolumn{3}{|c|}{$\begin{array}{c}\text { Maximum Centerline } \\
\text { Temperature }\end{array}$} & \multicolumn{3}{|c|}{$\begin{array}{l}\text { Maximum Cladding } \\
\text { Surface Temperature }\end{array}$} \\
\hline & & & & $\begin{array}{l}\text { Absolute } \\
\text { Reading } \\
(\mathrm{mm}) \\
\end{array}$ & $\begin{array}{c}\text { Relative } \\
\text { Increase } \\
(\mathrm{mm}) \\
\end{array}$ & $\begin{array}{r}\text { Absolute } \\
\text { Reading } \\
(\mathrm{K})\end{array}$ & $\begin{array}{l}\text { Relative } \\
\text { Increase } \\
\frac{(K)}{}\end{array}$ & $\begin{array}{c}\begin{array}{c}\text { Elevation of } \\
\text { Measurement } \\
(\mathrm{m})\end{array} \\
\end{array}$ & $\begin{array}{l}\text { Absolute } \\
\text { Reacing } \\
(\text { K })\end{array}$ & $\begin{array}{c}\text { Relative } \\
\text { Increase } \\
(\mathrm{K})\end{array}$ & $\begin{array}{c}\text { Elevation of } \\
\text { Measurement } \\
(\mathrm{m})\end{array}$ \\
\hline $\begin{array}{l}\text { IE-0i } 1 \\
0.1 \text { nm-gap } \\
\text { Irradiated cladding }\end{array}$ & 1.96 & 2.35 & 0.07 & 3.38 & 1.83 & 1867 & 180 & $\begin{array}{l}\text { Centerline } \\
\text { thermocouple } \\
\text { at } 0.74\end{array}$ & \multicolumn{2}{|c|}{$\begin{array}{c}\text { No indication of } \\
\text { increase }\end{array}$} & $0.62^{[\mathrm{a}]}$ \\
\hline $\begin{array}{l}\text { IE- } 0: 2 \\
0: 35-\mathrm{mm} \text { gap } \\
\text { Irradiated cladding }\end{array}$ & 4.79 & 7.44 & 0.32 & 0.56 & 0.08 & \multicolumn{2}{|c|}{$\begin{array}{l}\text { Failed at onset } \\
\text { of film boiling }\end{array}$} & -- & \multicolumn{2}{|c|}{$\begin{array}{c}\text { No indication of } \\
\text { increase }\end{array}$} & $0.62^{[\mathrm{a}]}$ \\
\hline $\begin{array}{l}\text { IE- } 013 \\
0.10-m m \text { gap } \\
\text { Unirradiated cladding }\end{array}$ & 4.68 & 6.57 & 0.18 & \multicolumn{2}{|c|}{ No measurement } & \multicolumn{2}{|c|}{$\begin{array}{l}\text { Fàled during } \\
\text { preconditioning }\end{array}$} & -- & $\begin{array}{c}850 \\
730 \\
1080 \\
\text { No indic } \\
\text { incr }\end{array}$ & $\begin{array}{r}180 \\
\text { rratic) } \\
90 \\
440 \\
\text { ation of } \\
\text { ease }\end{array}$ & $\begin{array}{l}0.52^{[\mathrm{b}]} \\
0.62^{[\mathrm{b}]} \\
0.62^{[\mathrm{b}, \mathrm{c}]} \\
0.72^{[\mathrm{b}]}\end{array}$ \\
\hline $\begin{array}{l}\text { IE-014 } \\
0.35-\mathrm{mm} \text { gap } \\
\text { Unirradiated cladding }\end{array}$ & 5.06 & 11.76 & 1.17 & 0.78 & 0.28 & $\begin{array}{l}\text { Faile } \\
\text { of } \mathrm{fi}\end{array}$ & $\begin{array}{l}\text { d at onset } \\
\text { Im boiling }\end{array}$ & -- & 1020 & 370 & $0.62^{[\mathrm{d}]}$ \\
\hline
\end{tabular}

[a] Spring-loaded thermocouples.

[b] Spaded junction, brazed in groove, Type $S$ thermocouple.

[c] Thermocouple failed after about $20 \mathrm{~s}$ film boiling.

[d] Spaded junction, welded in groove with braze fill, Type $S$ thermocouple. Thermocouples were located at the $0.52-$ and $0.72-m$ elevations on this fuel rod also but were not monitored on strip charts or did not show an increase. 


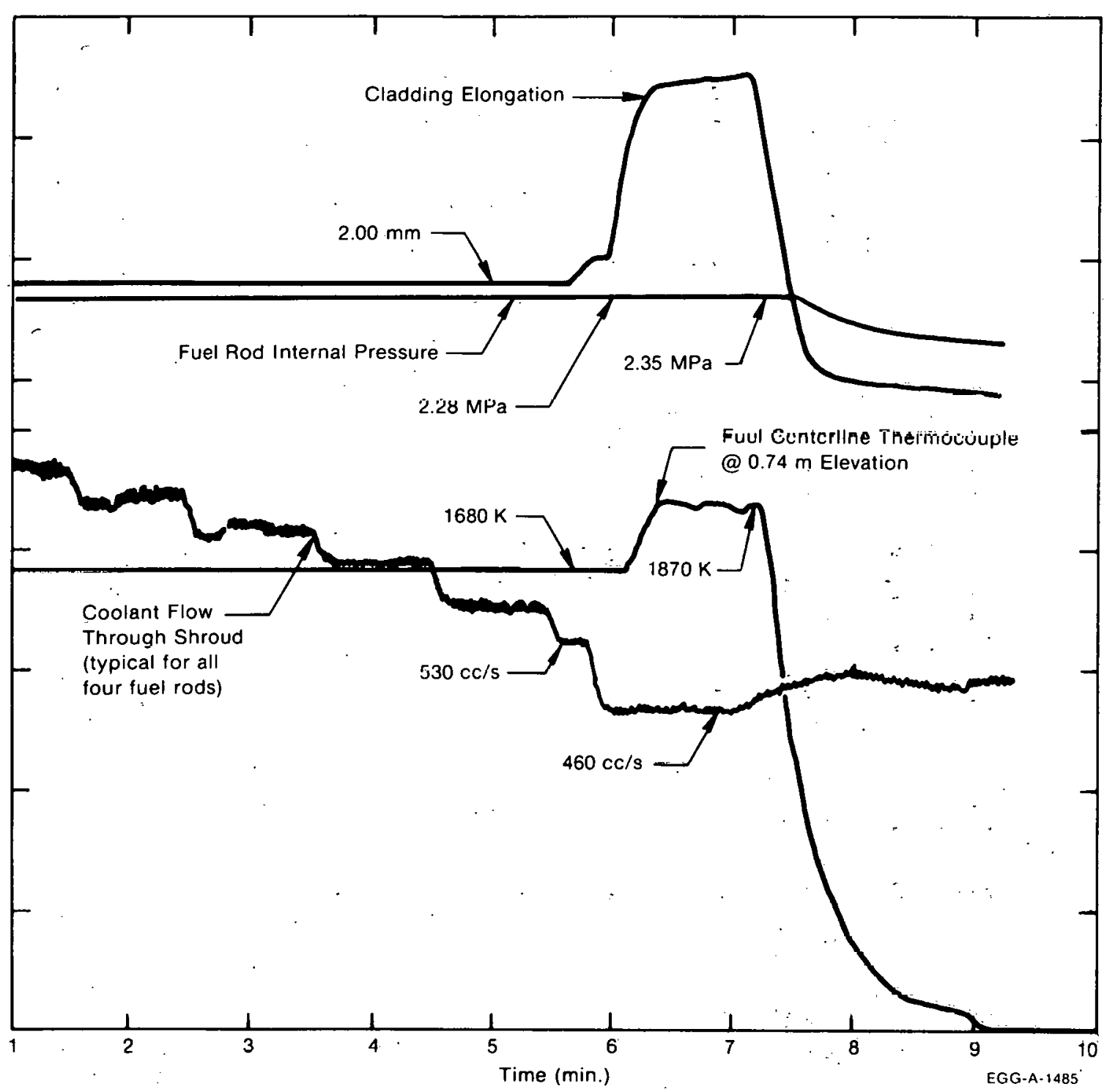

Fig. 47 Rod IE-011 strip chart data showing traces dufing flow reduction.

ultrasonic thermometer on Rod IE-014 failed during the preconditioning portion of the test. The remainder of the fuel centerline measurement devices worked at least until the onset of film boilung. ('The centerline thermocouple on Rod IE-011 functioned up to the end of the test.) Only two cladding surface thermocouples were determined to have failed: one failed during the preconditioning period; the other failed after experiencing $1080 \mathrm{~K}$ during film boiling. Spot checks of the other instruments indicated that they functioned throughout the test.

The overpower ramp the fuel rods were subjected to did not cause a pellet-cladding interaction failure, even where strong interaction occurred [as on Rod IE-011, which was fabricated with the small gap $(0.10 \mathrm{~mm})$ and irradiation damaged cladding]. No rod failures 


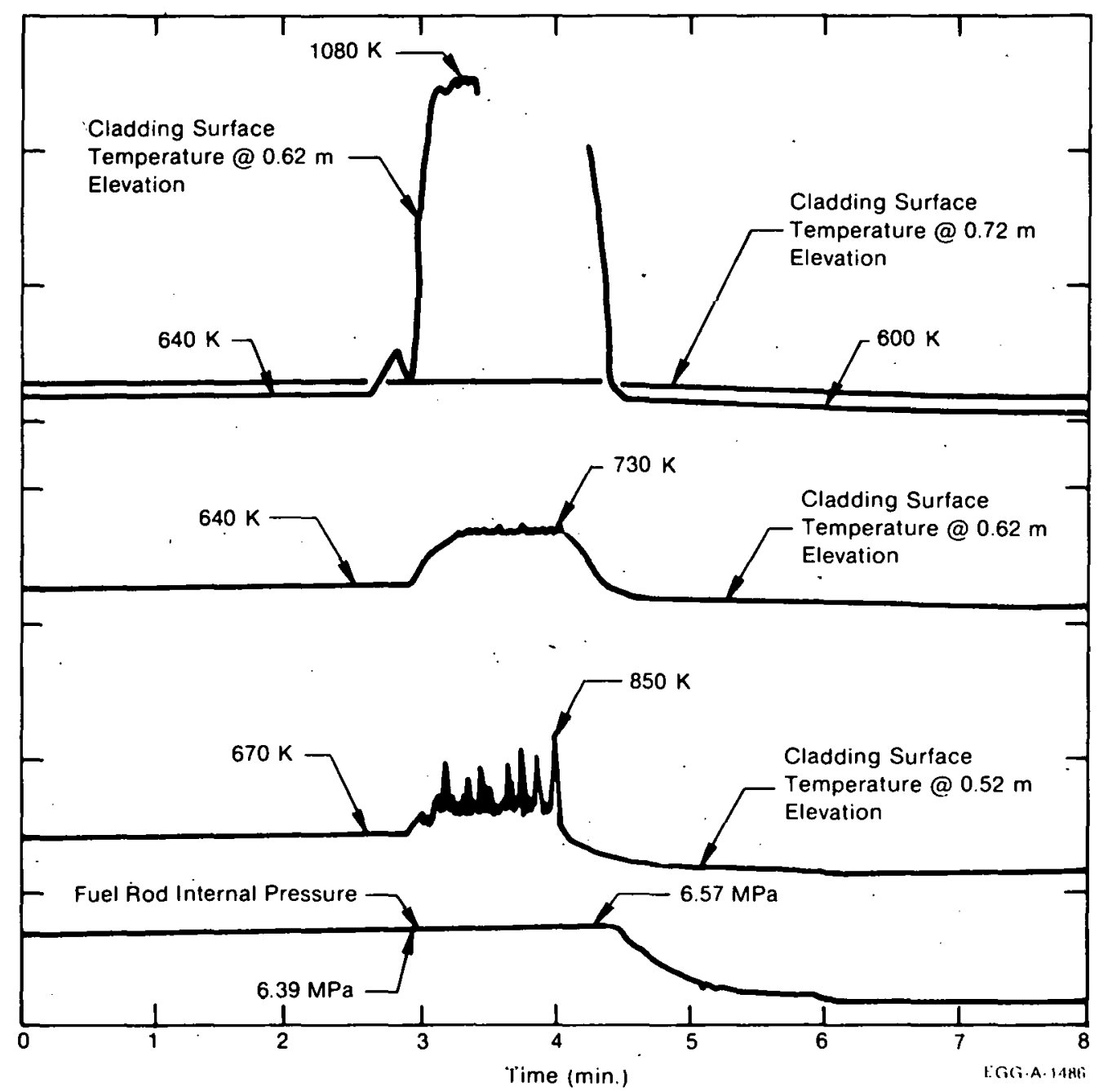

Fig. 48 Rod IE-013 strip chart data showing traces during flow reduction.

occurred during or after film boiling operation. On a macroscale, no evidence exists to suggest a difference in behavior between fuel rods clad with unirradiated or irradiated cladding.

\subsection{Irradiation Effects Test 3, Preliminary Results \\ D. W. Croucher}

The irradiation effects tests are performed to study the behavior of previously irradiated fuel rods subjected to overpower ramps that induce pellet-cladding interaction and film boiling operation.

Objectives of Irradiation. Effects Test 3 (IE-3) were to provide data on irradiated fuel rods with small variations in gap size and burnup. The data of interest include: 


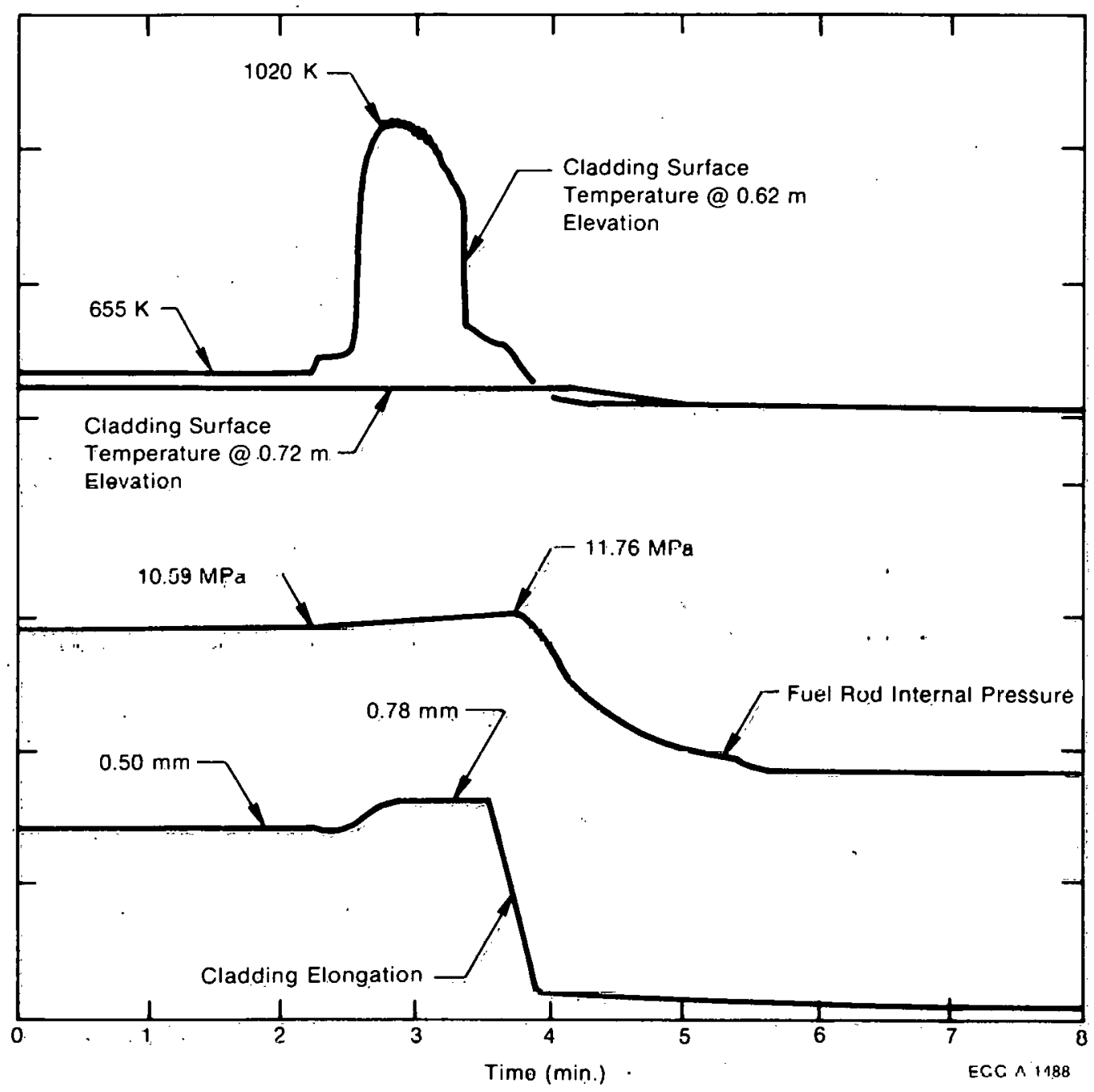

Fig. 49 Rod IE-014 strip chart data showing traces during flow reduction.

(1) Steady state data at various fuel rod power levels

(2) Data on fuel-cladding mechanical interaction during transient operating conditions

(3) Data for post-CHF fuel rod behavior at high power (approximately $66 \mathrm{~kW} / \mathrm{m}$ peak).

Preliminary results obtained from Test IE-3 are reviewed in this section.

1.4.1 Test Configuration. 'Test IE-3 was performed with four irradiated PWR-type fuel rods designated IE-015, IE-016, IE-017, and IE-018. These rods were previously irradiated in the Saxton reactor to burnups of $11,060,8550,14,780$, and $15,860 \mathrm{MWd} / \mathrm{t}$ for Rods IE-015, IE-016, IE-017, and IE-018, respectively. The rods were clad with zircaloy-4 tubing with a nominal outside diameter of $9.93 \mathrm{~mm}$ and a wall thickness of $0.592 \mathrm{~mm}$ prior to irradiation in the Saxton reactor. The original end caps were removed and replaced with instrumented end caps. 


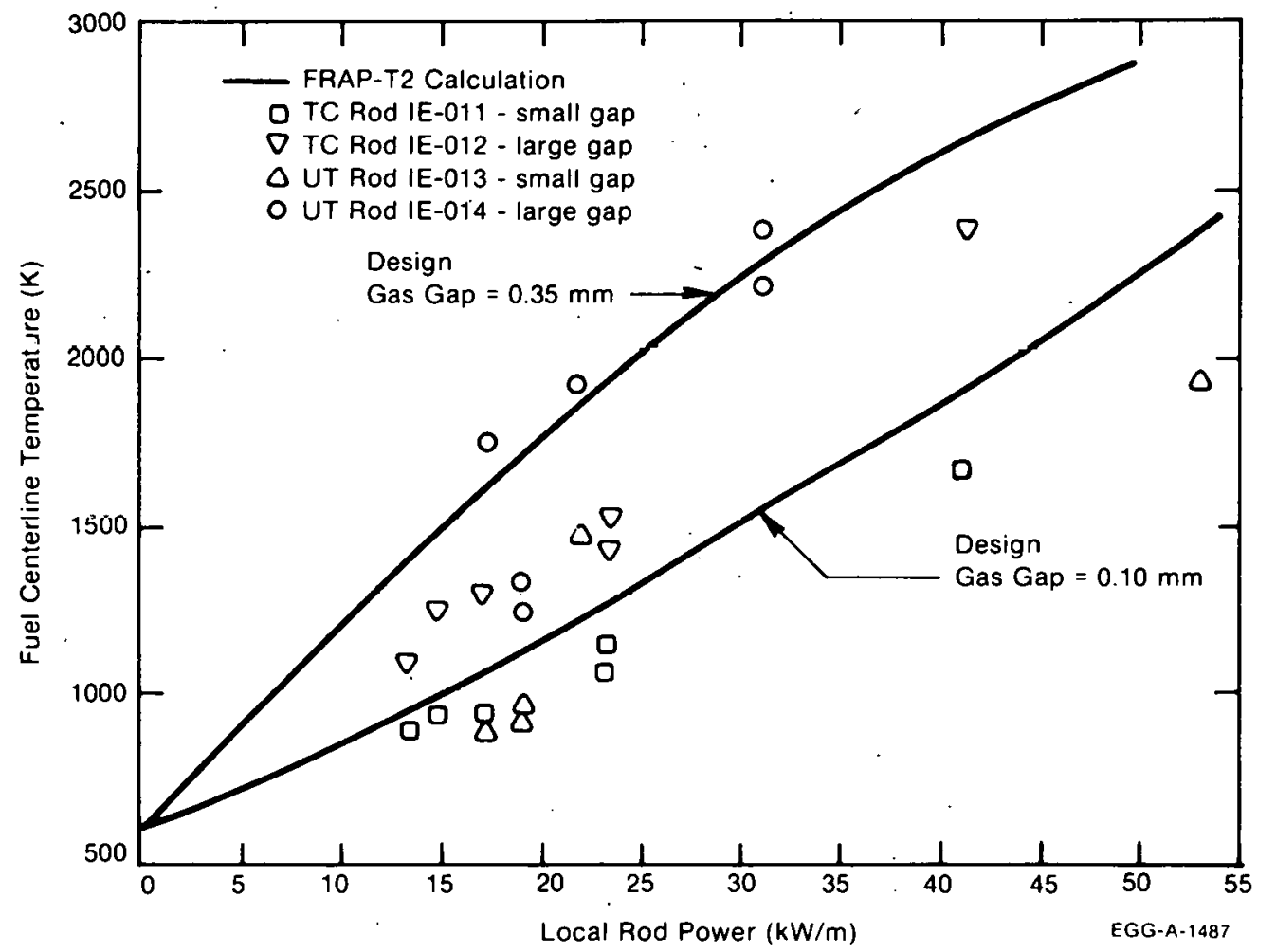

Fig. 50 Predicted and measured fuel centerline temperatures versus local fuel rod power for all four rods during the preconditioning and high-power portions of the test.

In Rods IE-015 and IE-016, additional fuel pellets were added to achieve the same stack length (about $890 \mathrm{~mm}$ ) as in Rods IE-017 and IE-018. Fuel pellets $(12.5 \mathrm{wt} \%$ uranium-235) were of a dished end design and $94 \%$ of theoretical density. Each rod was contained in a separate cylindrical flow shroud (16.3-mm inside diameter) and located in the PBF in-pile tube.

The rod and shroud assembly was instrumented to provide a thermal-hydraulic calibration of the fuel rod power. Each fuel rod was instrumented for measurement of internal gas pressure and cladding length change. Brazed thermocouples were mounted at several axial locations on Rod IE-108. Spring-loaded thermocouples were mounted on the cladding surface of Rod IE-017 at several elevations. A turbine flowmeter at the base of each shroud measured the fuel rod coolant flow rate. Copper-constantan thermocouples mounted on each shroud measured the coolant temperature rise. System pressure was monitored by two 69-MPa pressure transducers.

1.4.2 Test Conduct and Results. The test, conducted as shown in Figure 51 [15], included (a) a preconditioning period consisting of power calibration and gap conductance testing cycles, (b) a power increase, and (c) a flow reduction to induce film boiling on the rods. 

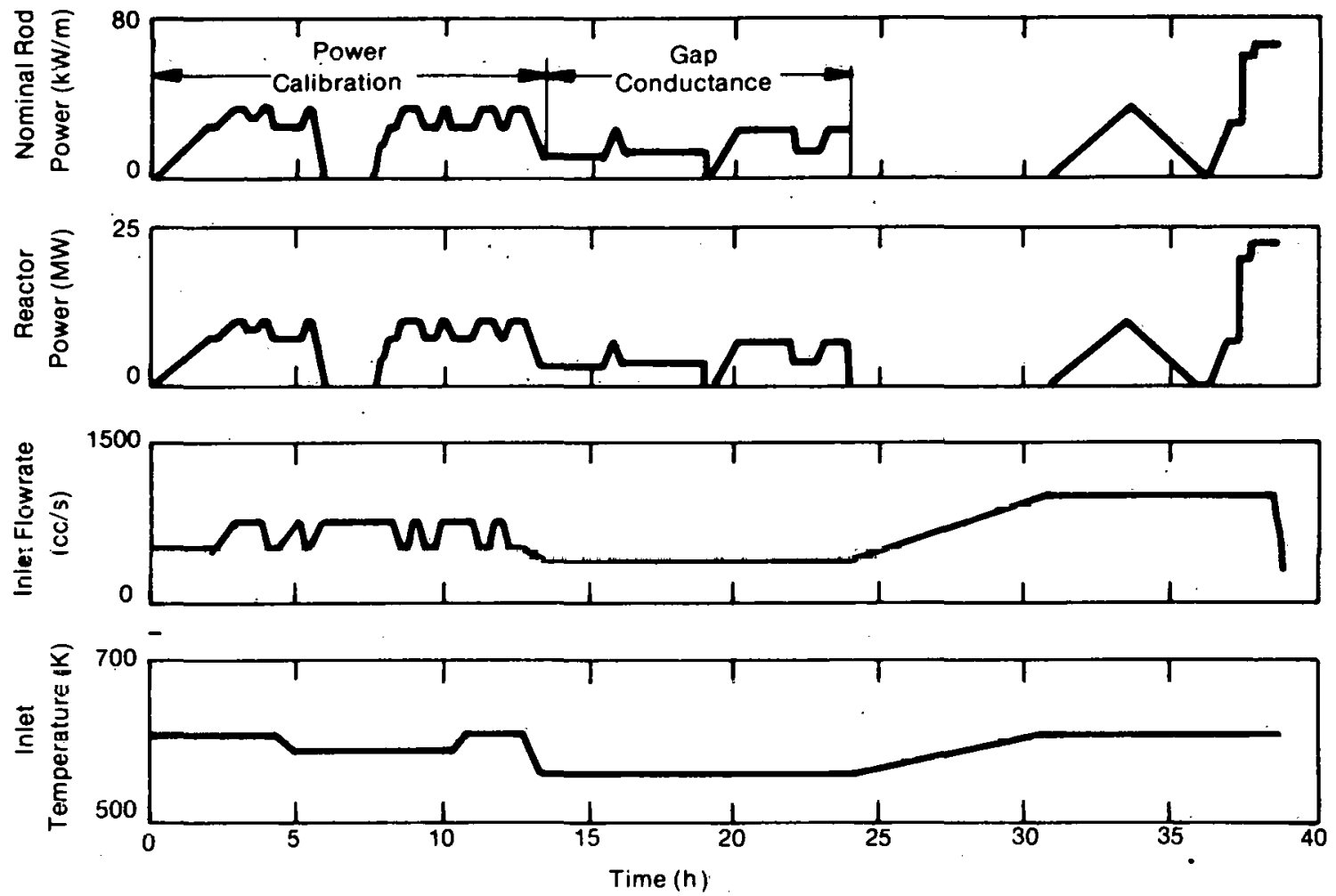

Fig. 51 Nominal operating conditions during Test IE-3.

(1) Preconditioning Period. The first series of power calibration cycles consisted of 16 steps at all possible combinations of two levels of flow rate $\left(505 \mathrm{~cm}^{3} / \mathrm{s}\right)$, inlet temperature (nominally 583 and $606 \mathrm{~K}$ ), and average fuel rod peak linear heat rating (nominally 23 and $33 \mathrm{~kW} / \mathrm{m}$ ). During the test, the relative rod power appeared to vary with reactor power level, control rod bank position, or both. The ratio of rod power to average rod power varied from a minimum of $1.003,0.997,1.009$, and 0.992 to a maximum of $1.003,0.975,1.054$, and 0.939 for Rod IE-015, IE-016, IE-017, and IE-018, respectively.

The gap conductance segment of the preconditioning period consisted of power oscillations of $\pm 20 \%$ at 10 and $18 \mathrm{~kW} / \mathrm{m}$ for two cycles.

Following the gap conductance testing, one additional cycle of preconditioning was completed. This period consisted of a power ramp from 0 to $33 \mathrm{~kW} / \mathrm{m}$ and then back to 0 at a nominal flow rate of $1000 \mathrm{~cm}^{3} / \mathrm{s}$. The ramp rate during this phase of the test was $0.23 \mathrm{~kW} / \mathrm{m}$-min and the inlet temperature was held at a nominal value of $606 \mathrm{~K}$.

(2) Power Increase. The preconditioning period ended with a steady state fuel rod power of $22 \mathrm{~kW} / \mathrm{m}$. The reactor was then increased at a ramp rate of $21 \mathrm{~kW} / \mathrm{m}-\mathrm{min}$ to provide an average fuel rod power of $61 \mathrm{~kW} / \mathrm{m}$. Once steady state was obtained at this power level, a calculation of fuel rod power using coolant flow and temperature differential indicated that the fuel rods had powers of $62.4,59.6,63.5$, and $57.9 \mathrm{~kW} / \mathrm{m}$ for Rods IE-015, IE-016, IE-017, and IE-018 respectively. After $20 \mathrm{~min}$, the reactor power was again increased by $2.5 \mathrm{MW}$ to bring the rod powers to $66.0,62.8,67.4$, and $60.4 \mathrm{~kW} / \mathrm{m}$, 
respectively. The fuel rod power, coolant inlet temperature, and coolant flow were held constant for approximately 40 additional minutes.

(3) Flow Reduction. The flow was reduced in $1-\mathrm{min}$ steps of $240 \mathrm{~kg} / \mathrm{s} \cdot \mathrm{m}^{2}$. At a mass flux of $2100 \mathrm{~kg} / \mathrm{s} \cdot \mathrm{m}^{2}$, the LVDT on Rod IE- 015 indicated the onset of film boiling. A subsequent flow reduction to $1620 \mathrm{~kg} / \mathrm{s} \cdot \mathrm{m}^{2}$ produced film boiling on the remaining rods. Because available cladding temperature measurements indicated a modest temperature rise, a final step flow reduction to $1370 \mathrm{~kg} / \mathrm{s} \cdot \mathrm{m}^{2}$ was performed. The flow was held constant for about $1 \mathrm{~min}$, after which the reactor was shut down.

Approximately $0.6 \mathrm{~min}$ after the beginning of shutdown, the pressure transducer on Rod IE-015 indicated rod failure. No fission product activity was indicated by the loop fission product detection system following the failure indication as had occurred during earlier tests with rod failure.

During loop depressurization, the pressure transducer on Rod IE-015 followed the coolant system pressure transducer, thus confirming rod failure. Approximately $2 \mathrm{~h}$ after test completion, the loop activity increased coincident with the faster rate of loop depressurization.

Preliminary data during the flow reduction portion of the test for Rod IE-018, typical of the other rods, are shown in Figure 52. The onset of film boiling was determined with good agreement between the rod instruments. The response of one of the cladding surface thermocouples at the $0.62-\mathrm{m}$ elevation was erratic during film boiling and may not have indicated the peak cladding temperature. The second and third flow reductions caused axial propagation of the region of film boiling as evidenced by the delayed response of the $0.52-\mathrm{m}$ thermocouple.

1.4.3 Discussion and Conclusions. The test was successfully completed with no apparent loss of critical instrumentation. Behavior of all four rods was monitored throughout the test. This discussion, however, is based on a limited number of strip-chart channels of data, and only partial conclusions as to performance can be made at this time.

The preconditioning period produced primarily steady state data. The power ramp of $21 \mathrm{~kW} / \mathrm{m}$-min to about $61 \mathrm{~kW} / \mathrm{m}$ produced substantial fuel-cladding mechanical interaction but did not cause rod failure.

The flow reduction transient produced film boiling at a flow rate of $430 \mathrm{~cm}^{3} / \mathrm{s}$, which can be compared with the predicted flow at critical heat flux (CHF) of $550 \mathrm{~cm}^{3} / \mathrm{s}$ [16]. The highest measured cladding surface temperature following CHF was lower than predicted ( 8.97 versus $1940 \mathrm{~K}$ ). Based on the erratic nature of the thermocouple response, thermocouple failure is suspected. Posttest examination of the fuel rods will be necessary to. establish the actual peak cladding temperature during this test. 


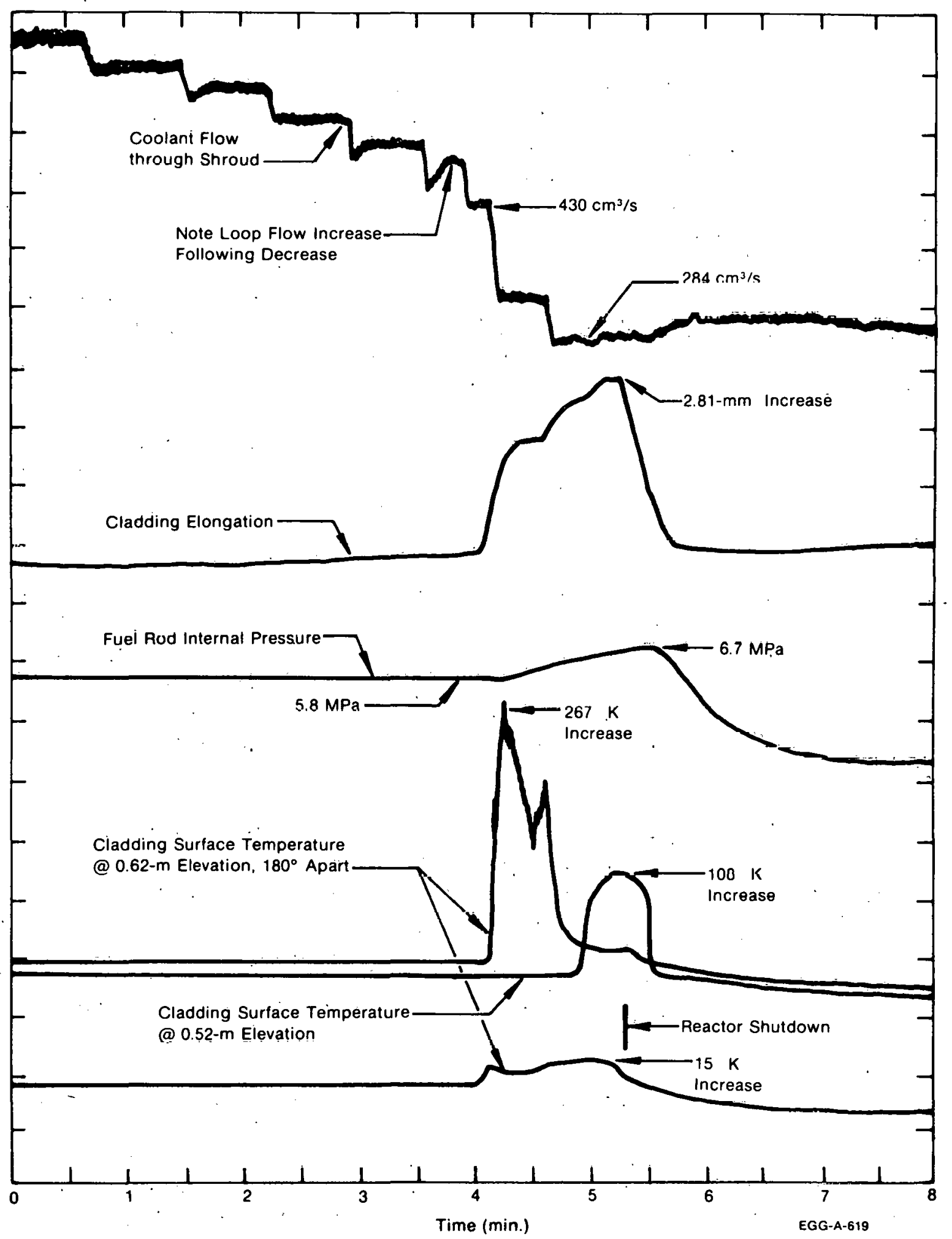

Fig. 52 Rod IE-018 strip chart data showing traces during flow reduction for internal pressure, cladding elongation, cladding surface temperature and flow rate. 
The pressure transducer on Rod IE-015 indicated rod failure approximately $0.6 \mathrm{~min}$ after the beginning of reactor shutdown. A very mild failure is suspected, because the loop fission product detection system did not respond until depressurization of the in-pile loop.

The steady state and transient data produced during Test IE-3 are considered useful for fuel code development and verification.

\subsection{Gap Conductance 'Test GC 2-1, Preliminary Results \\ R. W. Garner and D. T. Sparks}

Gap Conductance Test 2-1 (GC 2-1) is the first of a series of five tests designed to evaluate the effects of fuel density, initial gap width, and fill gas composition on the pellet-cladding gap conductance. The test series was based on a fractional factorial design to provide maximum information with a minimum number of tests.

Four boiling water reactor (BWR) type fuel rods were tested simultaneously in the PBF in-pile tube. Steady state and power oscillation data were obtained from which fuel rod gap conductance values will be determined as a function of test fuel rod linear power.

The objective of Gap Conductance Test Series 2 is to obtain experiment data for estimating fuel rod gap conductance values as a function of fuel rod linear power. The specific objectives of Test GC 2-1 were to:

(1) Obtain experiment data for estimating gap conductance values by both the steady state $\left(\int k d t\right)$ and power oscillation experiment methods.

(2) Obtain data to evaluate the effects of oscillation amplitude and frequency on the estimated gap conductance values for BWRdesign test fuel rods.

(3) Obtain data on four different design test fuel rods at the same time. This technique is in conformance with the objective of obtaining the maximum amount of information with a minimum number of tests.

(4) Obtain data to evaluate the ability of internal off-center thermocouples to provide more accurate fuel pellet surface temperature information than can be obtained by fuel centerline temperature measurements and associated calculations.

(5) Evaluate test result reproducibility by repeating some of the test conditions.

(6) Attempt to identify gap closure and opening conditions during power oscillations. 
1.5.1 Experiment Design. Test GC 2-1 was performed with four BWR-type fuel rods, each contained in a flow shroud $(19.3-\mathrm{mm}$ inside diameter) and located within the PBF in-pile tube. Test fuel rod designations were GC 501, GC 502, GC 503, and GC 504. Test instrumentation was provided to (a) evaluate the relationship between PBF core power and test fuel rod power, (b) estimate test fuel rod gap conductance values by the $\int \mathrm{kdt}$ method, using both centerline and off-centerline internal pellet temperature measurements, and (c) estimate test fuel rod gap conductance values by the power oscillation method. The test fuel rod and hardware nominal design characteristics are provided in Table $\mathrm{V}$.

Test measurement transducers included hardware instrumentation and fuel rod instrumentation. Hardware instrumentation consisted of flow meters, coolant $\Delta \mathrm{T}$ thermocouples, and coolant inlet thermocouples for determining test rod power densities hy calorimetric methods and cobalt axial flux wires for determining localized integrated power. Fuel rod instrumentation consisted of fuel centerline thermocouples, fuel off-center thermocouples, and cladding surface thermocouples for determining gap conductance values by both the $\int \mathrm{kdt}$ and power oscillation methods. The relative azimuthal and axial locations of the test rod thermocouples and orientations of the four test rods in the in-pile tube are shown in Figure. 53.

1.5.2 Experiment Conduct. Test GC 2-1 consisted of three parts: (a) steady state operation (power calibration) over a range of PBF core power levels during which each of the four test fuel rod power densities were determined and calibration constants between test rod power densities and core power were obtained, (b) a preconditioning period during which fuel pellet cracking and rearrangement effects on gap conductance values were evaluated, and (c) a power oscillation period during which the power was oscillated about several nominal power levels at a variety of oscillation magnitudes and periods.

The nominal coolant inlet temperature and pressure during the power calibration and preconditioning periods were $539 \mathrm{~K}$ and $7.17 \mathrm{MPa}$, respectively, with the inlet mass flux varying from 170 to $485 \mathrm{~cm}^{3} / \mathrm{s}$, depending on the test rod power densities. During the power oscillation portion of the test, the nominal coolant inlet temperature and pressure were $478 \mathrm{~K}$ and $7.17 \mathrm{MPa}$, respectively, with the inlet mass flux varying from approximately 470 to $600 \mathrm{~cm}^{3} / \mathrm{s}$, depending on the test rod power densities. Table VI provides a schedule of the range of oscillation conditions investigated during the power oscillation portion of the test. At each oscillation condition, the reactor was oscillated for approximately 30 cycles to obtain data sufficient to minimize statistical uncertainties.

Pretest calculations were performed with the FRAP-T ${ }^{[17]}$ computer code (MOD 002) to predict if gap closure would occur during the planned power oscillations and to indicate possible cladding surface temperature oscillation waveshape changes as a result of gap closure and opening during oscillations at certain power densities. Figures 54 and 55 provide the predicted responses of the cladding surface temperature and radial gap thickness, respectively, for $\pm 20 \%$ amplitude power oscillations performed on a helium filled BWR-design fuel rod with an initial $0.229-\mathrm{mm}$ diametral gap. The nominal test rod power density for Figures 54 and 55 is $46.0 \mathrm{~kW} / \mathrm{m}$. Figures 56 and 57 show the predicted responses of the same variables at a nominal test rod power density of $52.5 \mathrm{~kW} / \mathrm{m}$ and the same oscillation conditions. 
TABLE V

\section{NOMINAL DESIGN CHARACTERISTICS OF BWR-DESIGN TEST FUEL RODS FOR GAP CONDUCTANCE TEST GC 2-1}

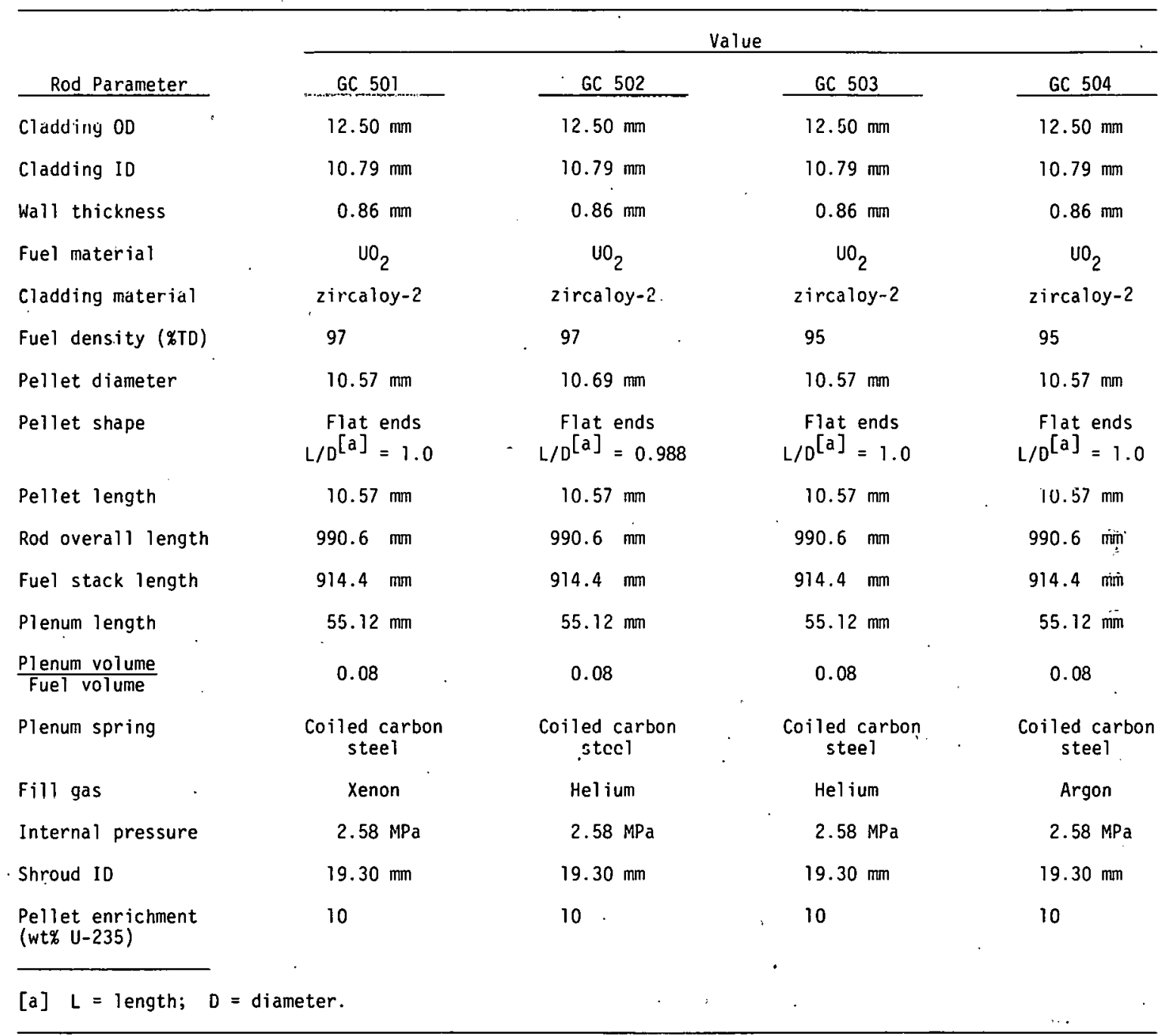




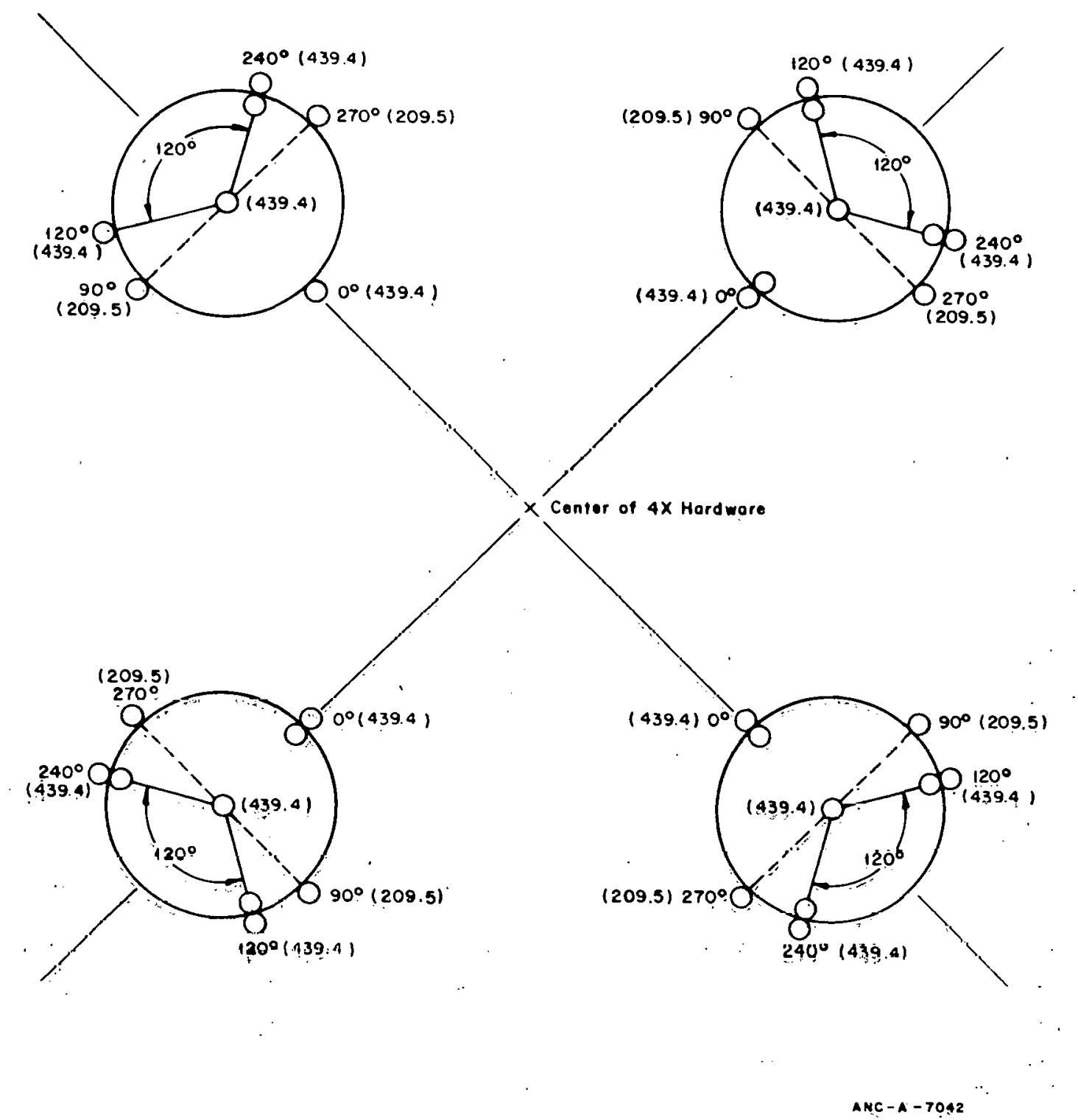

Fig. 53 Diagram of relative azimuthal and axial locations of thermocouples and orientation of test fuel rods in $4 \mathrm{X}$ hardware for Test GC 2-1.

Figure 54 shows a predicted sinusoidal response for the cladding surface temperature, and Figure 55 shows that the radial gap width changes with time but was predicted to not close during oscillations at the $46.0-\mathrm{kW} / \mathrm{m}$ nominal power level.

At the higher power density $(52.5 \mathrm{~kW} / \mathrm{m})$, the calculations predicted a significant change in the waveshape for the cladding surface temperature (Figure 56) and that the fuel-to-cladding gap would close on the increase in power and open on the decrease in power (Figure 57).

During the oscillation portion of the test, the cladding surface temperature oscillations were closely observed to determine if waveshape changes, possibly indicating gap closure and opening, could be observed. 
TABLE VI

OSCILLATION CONDITIONS DURING POWER OSCILLATION PORTION OF TEST GC 2-1

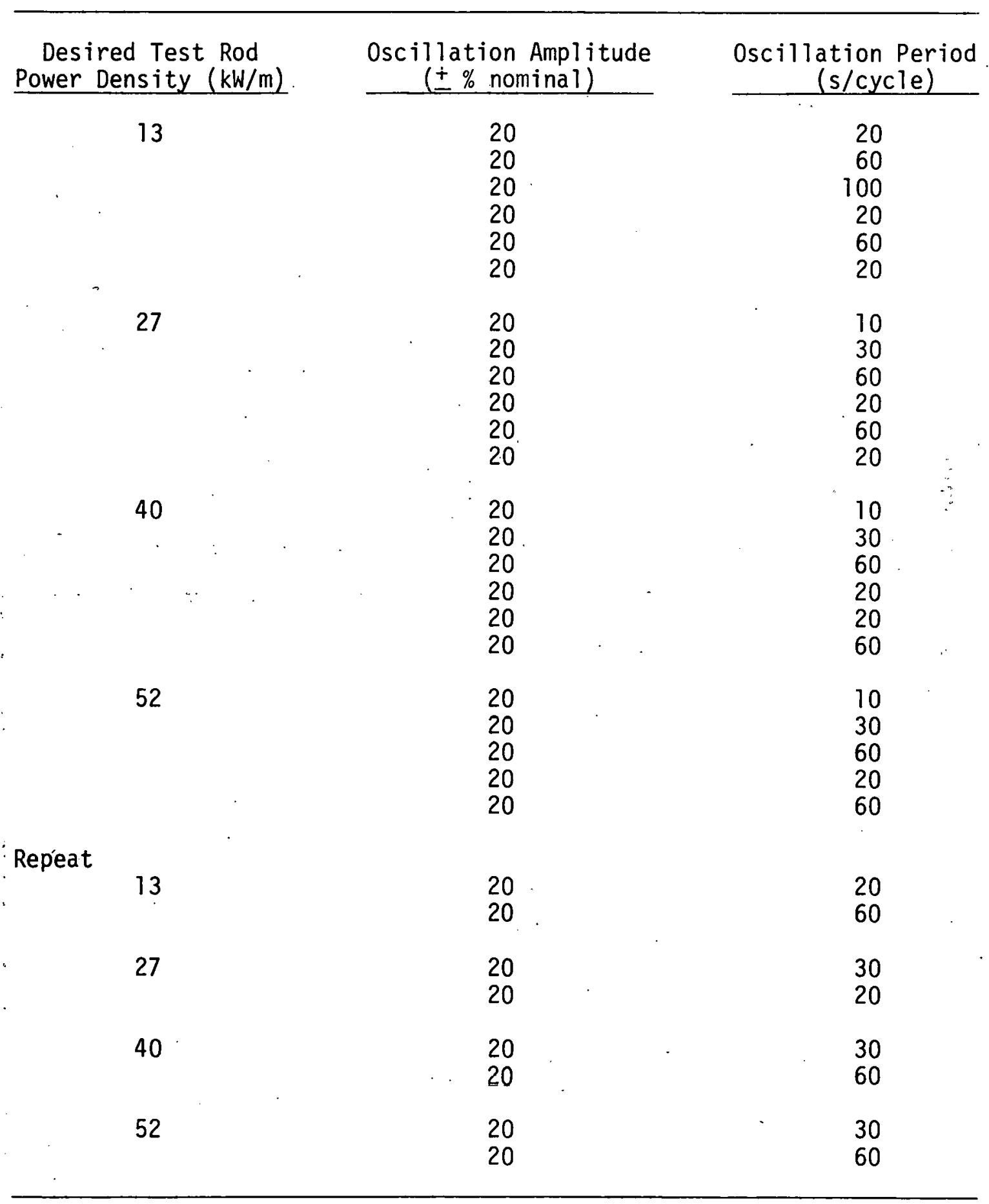




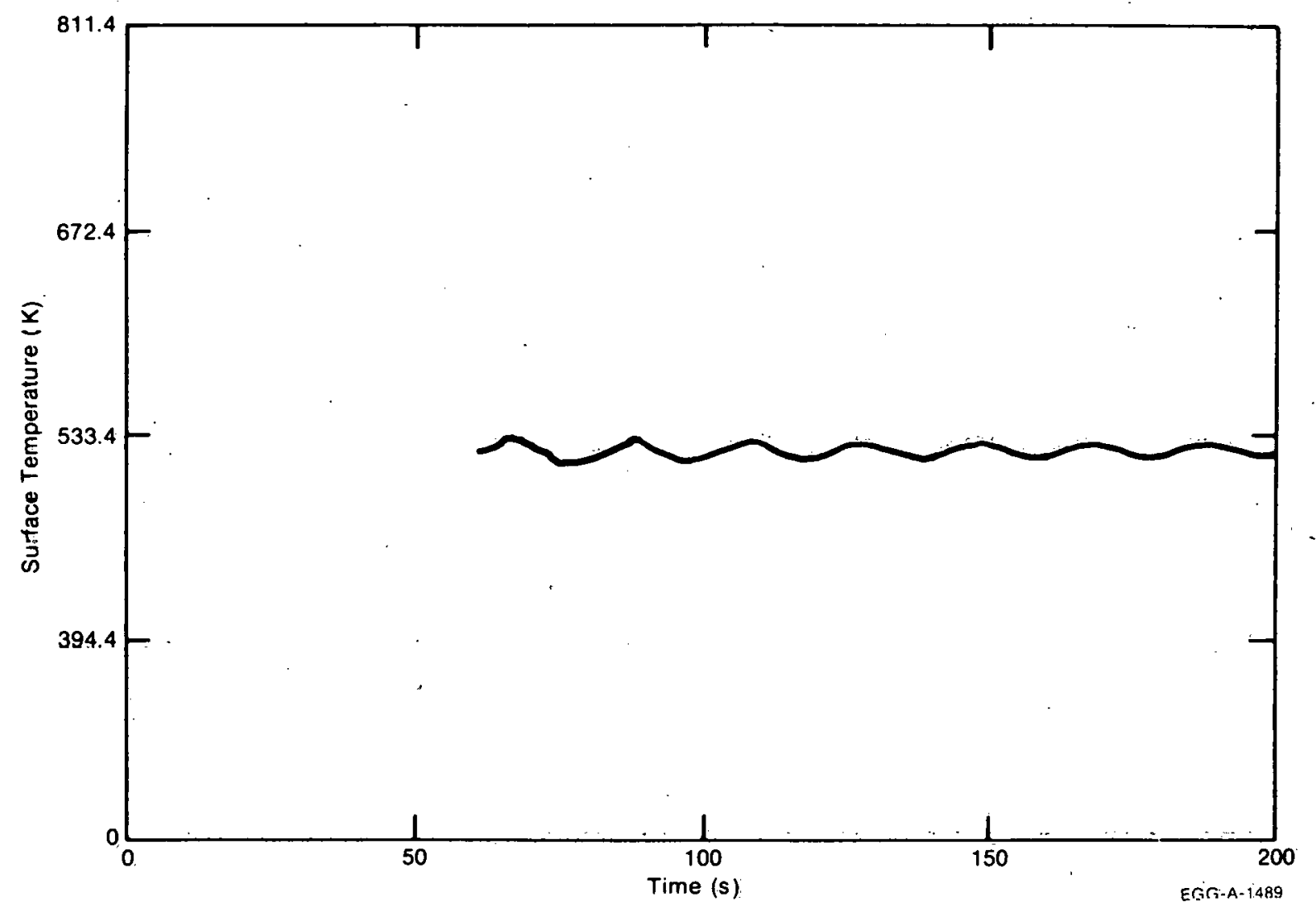

Fig. 54 Calculated cladding surface temperature oscillation for $\pm 20 \%$ amplitude, 20 s/cycle power oscillations at a nominal power density of $46 \mathrm{~kW} / \mathrm{m}$.

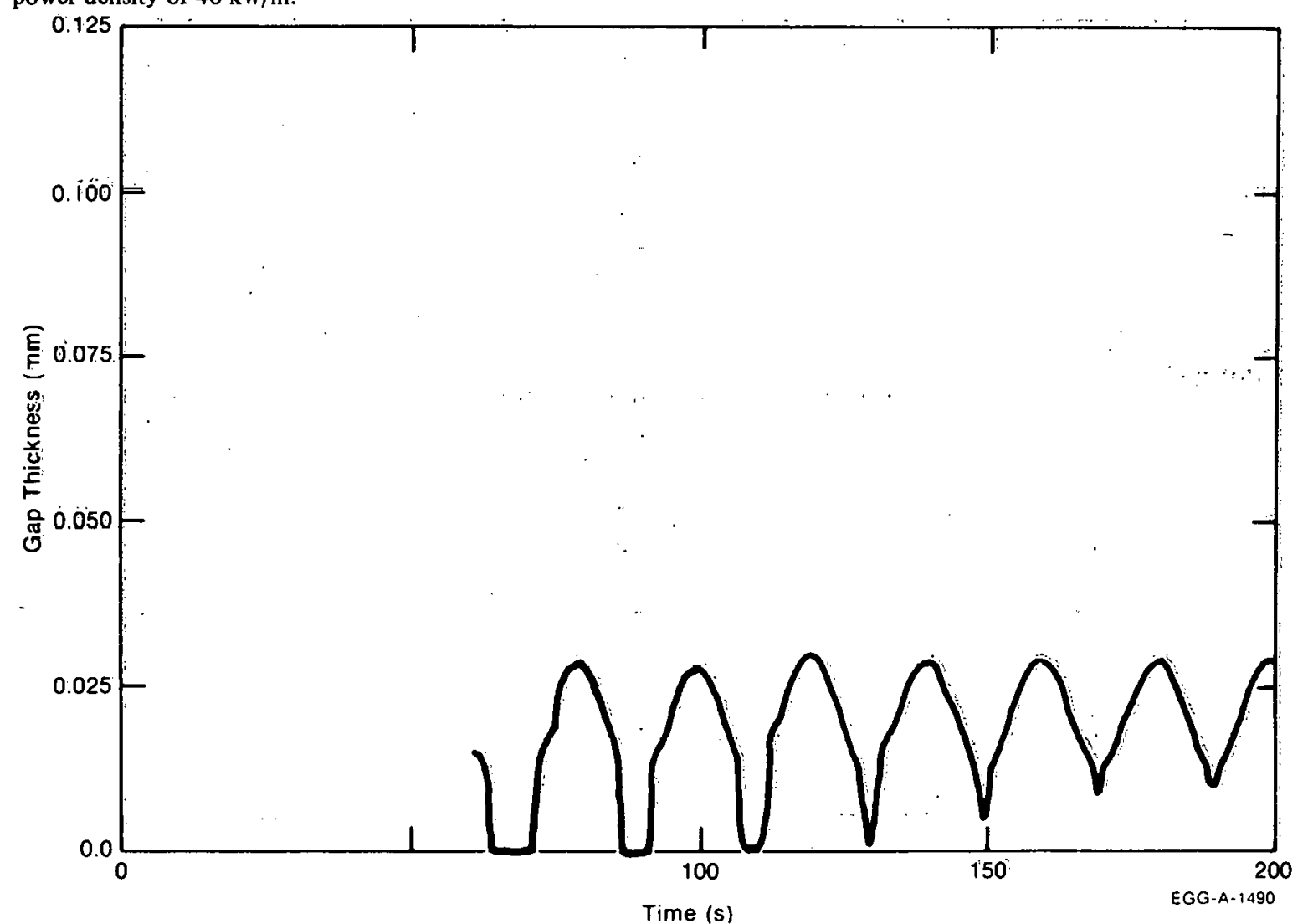

Fig. 55 Calculated gap thickness variations during $\pm 20 \%$ amplitude, 20 s/cycle power oscillations at a nominal power density of $46 \mathrm{~kW} / \mathrm{m}$. 


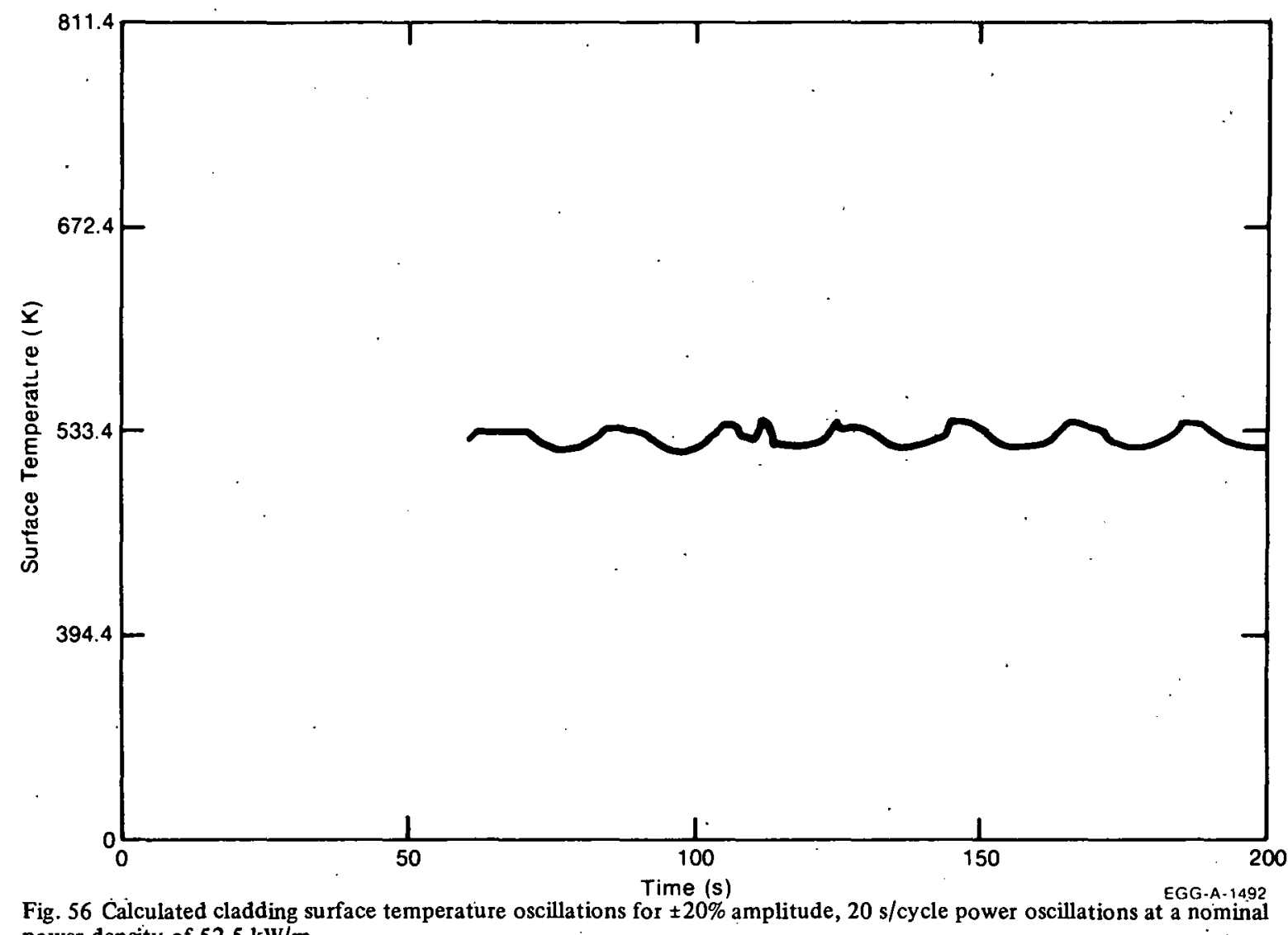
power density of $52.5 \mathrm{~kW} / \mathrm{m}$.

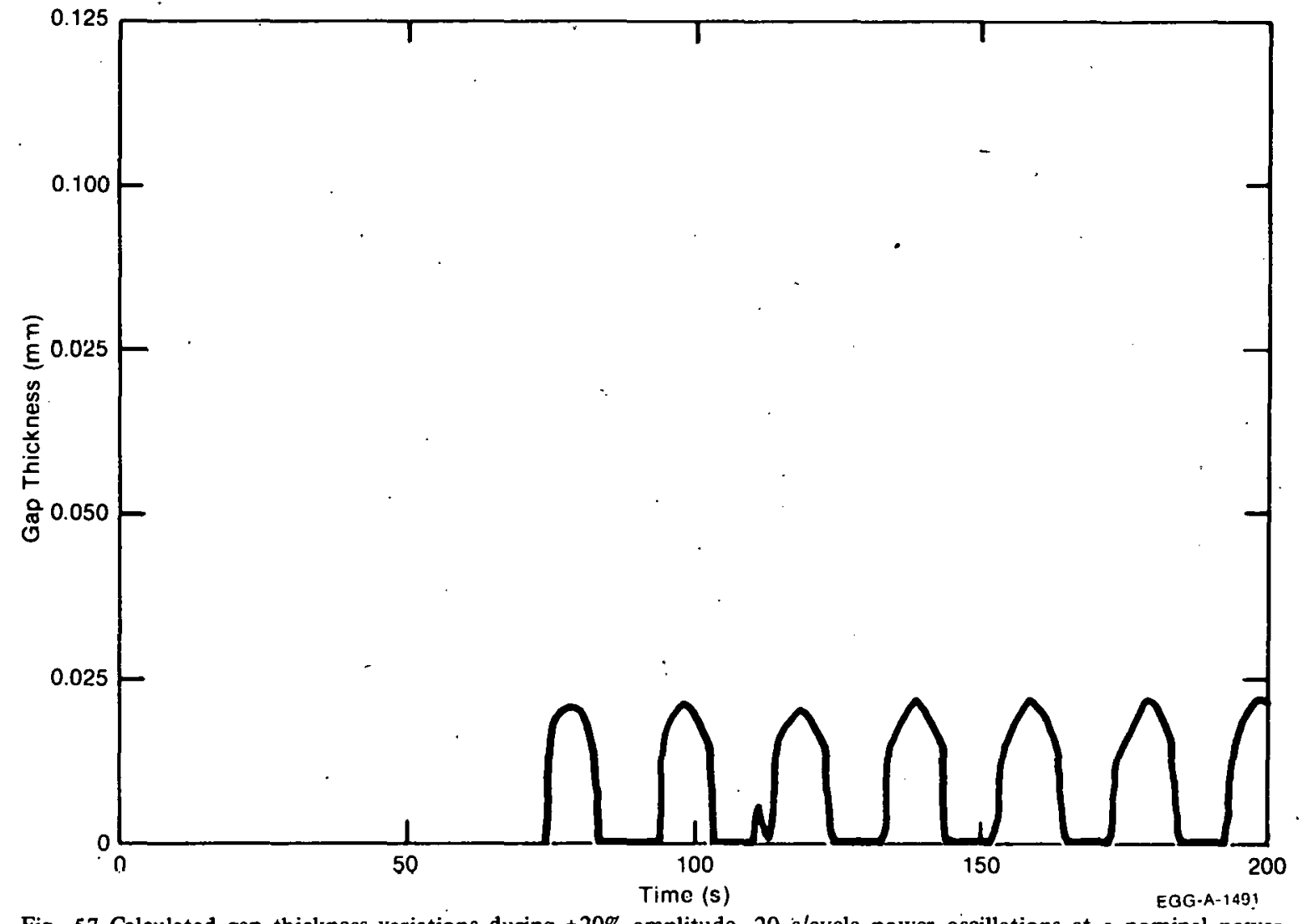

Fig. 57 Calculated gap thickness variations during $\pm 20 \%$ amplitude, $20 \mathrm{~s} /$ cycle power oscillations at a nominal power density of $52.5 \mathrm{~kW} / \mathrm{m}$.

69 
1.5.3 Experiment Results. During the increase in core power to initial criticality at $80 \mathrm{~kW}$, it was observed that the fuel temperatures in Rod GC 501, the xenon-filled rod, were significantly higher than the fuel temperatures in the other test rods. Higher temperatures were expected, but the indicated differences were larger than expected. As indicated in Reference 17, it was expected that the first power calibration point would be obtained at an indicated core power level of 3.1 MW. However, when the core power was increased to an indicated $2.65 \mathrm{MW}$ (corresponding to a nominal test rod peak power density of approximately $13 \mathrm{~kW} / \mathrm{m}$ ), the centerline thermocouple in Rod GC 501 indicated a temperature of approximately $1550 \mathrm{~K}$, and all three off-center thermocouples indicated temperatures of approximately $1440 \mathrm{~K}$ with high rates of temperature increase. It was postulated that at the indicated rates of temperature increase, the reliable temperature limit (approximately $1700 \mathrm{~K}$ ) of the off-center thermocouples would be exceeded before a core power of 3.1 MW could be obtained.

Rather than risk losing the off-center thermocouples in Rod GC 501 so early in the test, it was decided to obtain power calibration and power oscillation data at a few low power levels before increasing the core power above $2.65 \mathrm{MW}$. In addition, it was postulated that a reduction in power might cause the fuel pellets to crack, thereby closing the gap to some extent and possibly reducing the internal temperatures through increased heat transfer.

The core power was reduced in successive steps from $2.65 \mathrm{MW}$ to $2.0,1.5,1.0$, and $0.5 \mathrm{MW}$, and steady state data were obtained at each power level. The power was then increased to $1.6 \mathrm{MW}$ and power oscillations were performed at oscillation conditions of $\pm 10 \%$ amplitude and $0.05-\mathrm{Hz}$ frequency. The core power was then increased to $2.0 \mathrm{MW}$ and then to $2.9 \mathrm{MW}$, with oscillations performed at each power level. Power calibration data were also obtained at each steady state power level (nominal peak values of 7,9 , and $13 \mathrm{~kW} / \mathrm{m}$, respectively, in the test rods).

At the 2.9-MW core power level, the fuel temperatures in Rod GC 501 were still indicating high, with no evidence that pellet cracking had occurred. Following the oscillations at this power level, it was decided to increase core power to the highest possible level without exceeding the limit of the off-center thermocouples, as indicated on the on-line strip charts.

As the core power was increased slowly, the rate of temperature increase, as indicated by the centerline and off-center thermocouples in Rod GC 501, decreased. When the indicated core power was $5.0 \mathrm{MW}$, the centerline temperature was approximately $1880 \mathrm{~K}$ and the off-center temperatures ranged from 1360 to approximately $1700 \mathrm{~K}$; the thermocouples appeared to respond properly. At this time, the preconditioning portion of the test was performed by cycling the core power between 1.0 and 5.0 MW for three cycles.

Following the preconditioning portion of the test, the power oscillation portion was performed with only a few changes in the planned oscillation conditions. The specific changes were to eliminate oscillations at a frequency of $0.1 \mathrm{~Hz}$ and to add oscillations at 0.0166 and $0.01 \mathrm{~Hz}$. The objectives of the changes were to obtain information on the effect 
of oscillation frequency on the resulting gap conductance of the xenon-filled rod and to provide more time per cycle for heat transfer to obtain larger temperature increases on the cladding surface of the xenon rod.

(1) Power Calibration and Preconditioning. Listings of the on-line steady state data obtained during all three portions of the test (power calibration, preconditioning, and prior to each oscillation condition) are provided in Tables VII through $\mathrm{X}$ for each test fuel rod. The off-center temperature data have not been corrected for perturbation effects. Representative test fuel rod power calibration data, based on data in Tables VII through X, are shown in Table XI. Plots of the test fuel rod power as a function of SPND 3 current are shown in Figure 58. As shown in Table VIII and Figure 58, the relationship between test rod power and SPND current are quite linear but differ significantly from rod to rod, with Rod GC 502 having a consistently higher calibration factor than the other three rods. Rod GC 502 had the smallest initial gap width, indicating slightly larger fuel pellets (10.693-mm diameter compared with $10.566-\mathrm{mm}$ diameter) than the other rods. The effect of increased fuel volume in Rod GC 502 and the higher theoretical density in Rods GC 501 and GC 502 may be partly responsible for the differences seen in Figure 58.

The preliminary data obtained during the preconditioning period are also shown in Tables VII, VIII, IX, and X. These data do not indicate any significant changes in fuel centerline, off-center, or cladding surface temperatures with time that would result in identifiable fuel cracking or relocation effects on the gap conductance values.

Steady state data obtained during the power calibration and preconditioning periods and immediately prior to power oscillations at each power level will be used in estimating steady state gap conductance values. Some of the on-line data have been analyzed. Representative steady state gap conductance values using measured centerline temperatures are provided in Table XII.

(2) Power Oscillation. During the power oscillation portion of the test, on-line phase lag data were obtained, primarily with the cladding surface thermocouples, at $240^{\circ}$ on Rod GC 501 and GC 503. However, some data were also obtained on the other test rods. Representative measured phase lags and calculated power oscillation gap conductance values for each test rod are provided in Tables XIII through XVI for a range of test rod power densities and power oscillation conditions.

During power oscillation at the various power densities, the cladding surface temperature oscillations were closely observed in an attempt to detect changes in waveshape that may be indicative of gap closure, and possibly gap closure and opening, as predicted by the FRAP-T2 calculations. At power densities up to approximately $44 \mathrm{~kW} / \mathrm{m}$, the power oscillation responses of the cladding surface temperatures were all well defined sinusoids, representative traces of which are shown in Figures 59 and 60.

During oscillations at the planned highest power density of the "repeat" oscillations (as indicated by Table III), significant waveshape changes were observed for the cladding surface temperature oscillations on Rod GC 504 at an orientation of $0^{\circ}$. Observation of the 


\section{TABLE VII}

\section{PREL IMINARY ON-LINE STEADY STATE DATA FROM GAP CONDUCTANCE TEST GC 2-1 (ROD GC 501)}

\section{$539 \mathrm{~K}$ Inlet Temperature}

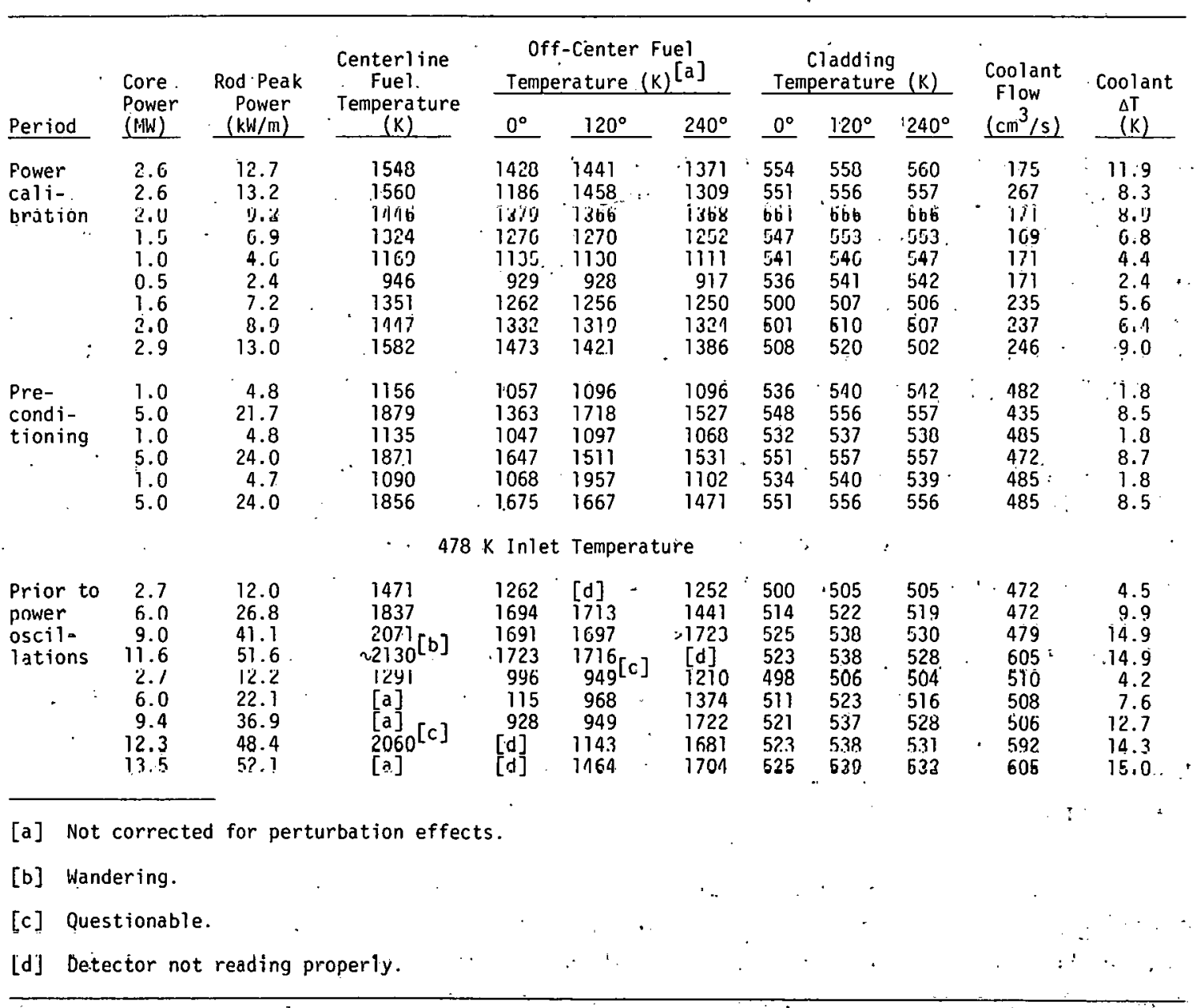


TABLE VIII

PREL IMINARY ON-LINE STEADY STATE DATA FROM
GAP CONDUCTANCE TEST GC 2-1 (ROD. GC 502)

$539 \mathrm{~K}$ Inlet Temperature

TABLE VIII

PRELIMINARY ON-LINE STEADY STATE DATA FROM GAP CONDUCTANCE TEST GC 2-1 (ROD GC 502)

$539 \mathrm{~K}$ Inlet Temperature

\begin{tabular}{|c|c|c|c|c|c|c|c|c|c|c|c|}
\hline \multirow[b]{2}{*}{ Period } & \multirow{2}{*}{$\begin{array}{l}\text { Core } \\
\text { Power } \\
\text { (MW) } \\
\end{array}$} & \multirow{2}{*}{$\begin{array}{l}\text { Rod Peak } \\
\text { Power } \\
(\mathrm{kW} / \mathrm{m}) \\
\end{array}$} & \multirow{2}{*}{$\begin{array}{l}\text { Centerl ine } \\
\text { Fuel } \\
\text { Temperature } \\
\text { (K) }\end{array}$} & \multicolumn{3}{|c|}{$\begin{array}{c}\text { Off-Center Fuel } \\
\text { Temperature }(K)^{[a]}\end{array}$} & \multicolumn{3}{|c|}{$\begin{array}{c}\text { Cladding } \\
\text { Temperature }(K) \\
\end{array}$} & \multirow{2}{*}{$\begin{array}{l}\text { Coolant } \\
\text { Flow } \\
\left(\mathrm{cm}^{3} / \mathrm{s}\right) \\
\end{array}$} & \multirow{2}{*}{$\begin{array}{l}\text { Coolan } \\
\Delta T \\
\text { (K) } \\
\end{array}$} \\
\hline & & & & $\underline{0^{\circ}}$ & $120^{\circ}$ & $\underline{240^{\circ}}$ & $\underline{0^{\circ}}$ & $120^{\circ}$ & $\underline{240^{\circ}}$ & & \\
\hline $\begin{array}{l}\text { Power } \\
\text { cali- } \\
\text { bration }\end{array}$ & $\begin{array}{l}2.6 \\
2.6 \\
2.0 \\
1.5 \\
1.0 \\
0.5 \\
1.6 \\
2.0 \\
2.9\end{array}$ & $\begin{array}{r}14.2 \\
14.5 \\
10.6 \\
.8 .1 \\
5.4 \\
2.9 \\
8.4 \\
10.4 \\
14.7\end{array}$ & $\begin{array}{l}875 \\
878 \\
789 \\
727 \\
661 \\
599 \\
681 \\
730 \\
837\end{array}$ & $\begin{array}{l}726 \\
725 \\
686 \\
655 \\
620 \\
574 \\
607 \\
631 \\
688\end{array}$ & $\begin{array}{l}{[b]} \\
{[b]} \\
{[b]} \\
{[b]} \\
{[b]} \\
{[b]} \\
{[b]} \\
{[b]} \\
{[b]} \\
{[b]}\end{array}$ & $\begin{array}{l}729 \\
729 \\
666 \\
627 \\
589 \\
547 \\
595 \\
619 \\
680\end{array}$ & $\begin{array}{l}558 \\
560 \\
559 \\
556 \\
548 \\
542 \\
509 \\
511 \\
521\end{array}$ & $\begin{array}{l}555 \\
557 \\
556 \\
551 \\
546 \\
541 \\
509 \\
.512 \\
514\end{array}$ & $\begin{array}{l}558 \\
556 \\
556 \\
551 \\
546 \\
541 \\
505 \\
506 \\
513\end{array}$ & $\begin{array}{l}201 \\
305 \\
203 \\
201 \\
201 \\
201 \\
271 \\
275 \\
285\end{array}$ & $\begin{array}{c}12 \\
8.3 \\
9.0 \\
6.9 \\
4.8 \\
.2 .7 \\
5.6 \\
6.8 \\
9.1\end{array}$ \\
\hline $\begin{array}{l}\text { Pre- } \\
\text { condi- } \\
\text { tioning }\end{array}$ & $\begin{array}{l}1.0 \\
5.0 \\
1.0 \\
5.0 \\
1.0 \\
5.0\end{array}$ & $\begin{array}{r}5.5 \\
25.2 \\
5.6 \\
25.3 \\
5.6 \\
25.2\end{array}$ & $\begin{array}{r}653 \\
1130 \\
646 \\
1148 \\
649 \\
1130\end{array}$ & $\begin{array}{l}607 \\
828 \\
602 \\
826 \\
602 \\
826\end{array}$ & $\begin{array}{l}462 \\
{[\mathrm{~b}]} \\
462 \\
{[\mathrm{~b}]-} \\
464 \\
{[\mathrm{~b}]}\end{array}$ & $\begin{array}{l}588 \\
843 \\
580 \\
843 \\
583 \\
840\end{array}$ & $\begin{array}{l}542 \\
565 \\
539 \\
566 \\
541 \\
566\end{array}$ & $\begin{array}{l}541 \\
555 \\
538 \\
557 \\
539 \\
556\end{array}$ & $\begin{array}{l}541 \\
555 \\
538 \\
555 \\
540 \\
555\end{array}$ & $\begin{array}{l}542 \\
548 \\
544 \\
535 \\
548 \\
548\end{array}$ & $\begin{array}{l}2.1 \\
8.2 \\
2.1 \\
8.4 \\
2.1 \\
8.2\end{array}$ \\
\hline \multicolumn{7}{|c|}{$.478 \mathrm{~K} \mathrm{Inlet} \mathrm{Temperature}$} & & & & & \\
\hline $\begin{array}{l}\text { Prior to } \\
\text { power } \\
\text { oscil- } \\
\text { lations }\end{array}$ & $\begin{array}{r}2.7 \\
6.0 \\
9.0 \\
11.6 \\
2.7 \\
6.0 \\
9.4 \\
12.3 \\
13.5\end{array}$ & $\begin{array}{l}13.6 \\
27.7 \\
41.5 \\
51.8 \\
13.5 \\
27.9 \\
41.8 \\
53.0 \\
56.8\end{array}$ & $\begin{array}{r}794 \\
1135 \\
1359 \\
{[\mathrm{~b}]} \\
{[\mathrm{b}]} \\
{[\mathrm{b}]} \\
{[\mathrm{b}]} \\
\mathrm{b}] \\
{[\mathrm{b}]} \\
\mathrm{b}]\end{array}$ & $\begin{array}{l}{[b]} \\
{[b]} \\
{[b]} \\
{[b]} \\
{[b]} \\
{[b]} \\
{[b]} \\
{[b]} \\
{[b]} \\
{[b]}\end{array}$ & $\begin{array}{l}{[b]} \\
{[b]} \\
{[b]} \\
{[b]} \\
{[b]} \\
{[b]} \\
{[b]} \\
{[b]} \\
{[b]} \\
{[b]}\end{array}$ & $\begin{array}{l}{[b]} \\
{[b]} \\
{[b]} \\
{[b]} \\
{[b]} \\
{[b]} \\
{[b]} \\
{[b]} \\
{[b]} \\
{[b]}\end{array}$ & $\begin{array}{l}509 \\
532 \\
546 \\
545 \\
506 \\
520 \\
.535 \\
542 \\
544\end{array}$ & $\begin{array}{l}506 \\
518 \\
530 \\
533 \\
504 \\
516 \\
527 \\
532 \\
534\end{array}$ & $\begin{array}{l}505 \\
516 \\
526 \\
528 \\
503 \\
554 \\
525 \\
529 \\
531\end{array}$ & $\begin{array}{l}529 \\
531 \\
538 \\
680 \\
567 \\
567 \\
568 \\
661 \\
674\end{array}$ & $\begin{array}{r}4.8 \\
9.4 \\
13.7 \\
13.6 \\
4.5 \\
8.9 \\
13.1 \\
14.3 \\
15.0\end{array}$ \\
\hline [u] & & Per & secior & & & & & & & & \\
\hline [b] & & cated & rking $p$ & rly. & & & & & & & \\
\hline
\end{tabular}


TABLE IX

PRELIMINARY ON-LINE STEADY STATE DATA FROM

GAP CONDUCTANCE TEST GC 2-1. (ROD GC 503)

$539 \mathrm{~K}$ Inlet Temperature

\begin{tabular}{|c|c|c|c|c|c|c|c|c|c|c|c|}
\hline \multirow[b]{2}{*}{ Period } & \multirow{2}{*}{$\begin{array}{l}\text { Core } \\
\text { Power } \\
\text { (MW) } \\
\end{array}$} & \multirow{2}{*}{$\begin{array}{c}\text { Rod Peak } \\
\text { Power } \\
(\mathrm{kW} / \mathrm{m}) \\
\end{array}$} & \multirow{2}{*}{$\begin{array}{c}\text { Centerline } \\
\text { Fuel } \\
\text { Temperature } \\
(\mathrm{K}) \\
\end{array}$} & \multicolumn{3}{|c|}{$\begin{array}{c}\text { Off-Center Fuel } \\
\text { Temperature (K) }\end{array}$} & \multicolumn{3}{|c|}{$\begin{array}{l}\text { Cladding } \\
\text { Temperature }(K)\end{array}$} & \multirow{2}{*}{$\begin{array}{l}\text { Coolant } \\
\text { Flow } \\
\left(\mathrm{cm}^{3} / \mathrm{s}\right)\end{array}$} & \multirow{2}{*}{$\begin{array}{c}\text { Coolant } \\
\Delta \mathrm{T} \\
(\mathrm{K}) \\
\end{array}$} \\
\hline & & & & $0^{\circ}$ & $120^{\circ}$ & $\underline{240^{\circ}}$ & $0^{\circ}$ & $\underline{120^{\circ}}$ & $\underline{240^{\circ}}$ & & \\
\hline $\begin{array}{l}\text { Power } \\
\text { cali- } \\
\text { bration }\end{array}$ & $\begin{array}{l}2.5 \\
2.6 \\
2.0 \\
1.6 \\
1.0 \\
0.5 \\
1.6 \\
2.1 \\
2.9\end{array}$ & $\begin{array}{r}9.3 \\
13.2 \\
3.8 \\
7.3 \\
4.8 \\
2.4 \\
7.3 \\
9.8 \\
13.1\end{array}$ & $\begin{array}{l}948 \\
957 \\
B 03 \\
787 \\
710 \\
638 \\
839 \\
796 \\
916\end{array}$ & $\begin{array}{l}817 \\
814 \\
7597 \\
697 \\
613 \\
588 \\
655 \\
645 \\
781\end{array}$ & $\begin{array}{l}783 \\
781 \\
733 \\
600 \\
613 \\
592 \\
650 \\
548 \\
752\end{array}$ & $\begin{array}{l}711 \\
744 \\
70 ? \\
604 \\
619 \\
577 \\
658 \\
685 \\
736\end{array}$ & $\begin{array}{l}565 \\
556 \\
356 \\
553 \\
517 \\
542 \\
506 \\
508 \\
514\end{array}$ & $\begin{array}{l}558 \\
559 \\
558 \\
554 \\
518 \\
543 \\
507 \\
509 \\
516\end{array}$ & $\begin{array}{l}558 \\
557 \\
657 \\
552 \\
547 \\
542 \\
506 \\
608 \\
515\end{array}$ & $\begin{array}{l}140 \\
302 \\
280 \\
203 \\
209 \\
206 \\
274 \\
275 \\
283\end{array}$ & $\begin{array}{l}11 \\
7.3 \\
7.7 \\
5.8 \\
3.8 \\
1.9 \\
4.5 \\
5.7 \\
7.9\end{array}$ \\
\hline $\begin{array}{l}\text { Pre- } \\
\text { condi- } \\
\text { tioning } \\
\text { ting }\end{array}$ & $\begin{array}{l}1.0 \\
5.0 \\
1.0 \\
5.0 \\
1.0 \\
5.0\end{array}$ & $\begin{array}{r}5.3 \\
21.7 \\
5.3 \\
21.8 \\
5.3 \\
22.3\end{array}$ & $\begin{array}{r}698 \\
1232 \\
693 \\
1234 \\
718 \\
1232\end{array}$ & $\begin{array}{l}635 \\
961 \\
631 \\
968 \\
633 \\
971\end{array}$ & $\begin{array}{l}633 \\
917 \\
631 \\
910 \\
631 \\
908\end{array}$ & $\begin{array}{r}635 \\
\cdot \quad 842 \\
637 \\
845 \\
633 \\
6 \quad 848\end{array}$ & $\begin{array}{l}541 \\
554 \\
538 \\
555 \\
540 \\
555\end{array}$ & $\begin{array}{l}542 \\
557 \\
539 \\
558 \\
542 \\
558\end{array}$ & $\begin{array}{l}541 \\
558 \\
538 \\
558 \\
541 \\
558\end{array}$ & $\begin{array}{l}529 \\
529 \\
529 \\
516 \\
535 \\
539\end{array}$ & $\begin{array}{r}1.7 \\
\cdot 7.0 \\
1.7 \\
7.2 \\
1.7 \\
7.1\end{array}$ \\
\hline \multicolumn{12}{|c|}{$478 \mathrm{~K}$ Inlet Temperature } \\
\hline $\begin{array}{l}\text { Prior to } \\
\text { power } \\
\text { osril- } \\
\text { lations }\end{array}$ & $\begin{array}{r}2.7 \\
6.0 \\
9.0 \\
11.6 \\
2.7 \\
6.0 \\
9.4 \\
12.3 \\
13.5\end{array}$ & $\begin{array}{l}12.5 \\
24.3 \\
35.2 \\
45.6 \\
12.6 . \\
24.6 \\
35.5 \\
45.5 \\
48.8\end{array}$ & $\begin{array}{r}867 \\
1228 \\
1573 \\
1870 \\
789 \\
1140 \\
1489 \\
1821 \\
1936\end{array}$ & $\begin{array}{c}740 \\
958 \\
1072 \\
1163 \\
697 \\
908 \\
1092 \\
1223 \\
1148\end{array}$ & $\begin{array}{r}738 \\
886 \\
976 \\
1122 \\
681 \\
893 \\
1049 \\
1145 \\
1186\end{array}$ & $\begin{array}{c}768 \\
908 \\
1041 \\
1093 \\
753 \\
961 . \\
1102 \\
1199 \\
1181\end{array}$ & $\begin{array}{l}506 \\
516 \\
527 \\
530 \\
503 \\
516 \\
526 \\
530 \\
533\end{array}$ & $\begin{array}{l}507 \\
521 \\
534 \\
537 \\
505 \\
518 \\
530 \\
536 \\
536\end{array}$ & $\begin{array}{l}507 \\
520 \\
533 \\
535 \\
505 \\
516 \\
527 \\
533 \\
536\end{array}$ & $\begin{array}{r}511 \\
510 \\
516 \\
655 \\
548 \\
550 \\
548 \\
640 \\
665\end{array}$ & $\begin{array}{r}4.2 \\
8.2 \\
.11 .8 \\
12.1 \\
4.0 \\
7.7 \\
11.2 \\
12.3 \\
13.0\end{array}$ \\
\hline
\end{tabular}

[a] Not corrected for perturbation effects. 
TABLE X

PREL IMINARY ON-LINE STEADY STATE DATA FROM

GAP CONDUCTANCE TEST GC 2-1 (ROD GC 504)

$539 \mathrm{~K}$ Inlet Temperature

\begin{tabular}{|c|c|c|c|c|c|c|c|c|c|c|c|}
\hline \multirow[b]{2}{*}{ Period } & \multirow{2}{*}{$\begin{array}{l}\text { Core } \\
\text { Power } \\
\text { (MW) }\end{array}$} & \multirow{2}{*}{$\begin{array}{c}\text { Rod Peak } \\
\text { Power } \\
(\mathrm{kW} / \mathrm{m})\end{array}$} & \multirow{2}{*}{$\begin{array}{c}\text { Centerline } \\
\text { Fuel } \\
\text { Tempera ture } \\
\text { (K) }\end{array}$} & \multicolumn{3}{|c|}{$\begin{array}{l}\text { Off-Center Fuel } \\
\text { Temperature }(\mathrm{K})^{[\mathrm{a}]}\end{array}$} & \multicolumn{3}{|c|}{$\begin{array}{c}\text { Cladding } \\
\text { Temperature }(\mathrm{K}) \\
\end{array}$} & \multirow{2}{*}{$\begin{array}{l}\text { Coolant } \\
\text { Flow } \\
\left(\mathrm{cm}^{3} / \mathrm{s}\right)\end{array}$} & \multirow{2}{*}{$\begin{array}{c}\text { Coolant } \\
\Delta \mathrm{T} \\
(\mathrm{K}) \\
\end{array}$} \\
\hline & & & & $0^{\circ}$ & $12.0^{\circ}$ & $\underline{240^{\circ}}$ & $0^{\circ}$ & $120^{\circ}$ & $\underline{240^{\circ}}$ & & \\
\hline $\begin{array}{l}\text { Power } \\
\text { cali- } \\
\text { bration }\end{array}$ & $\begin{array}{l}2.6 \\
2.6 \\
2.0 \\
1.5 \\
1.0 \\
0.5 \\
1.6 \\
2.0 \\
2.9\end{array}$ & $\begin{array}{r}12.3 \\
12.3 \\
9.3 \\
7.0 \\
4.7 \\
2.5 \\
6.9 \\
8.8 \\
12.7\end{array}$ & $\begin{array}{r}1342 \\
1366 \\
1247 \\
1121 \\
979 \\
794 \\
1130 \\
1235 \\
1355\end{array}$ & $\begin{array}{c}\text { [b] } \\
-- \\
-- \\
-- \\
-- \\
-- \\
-- \\
--\end{array}$ & $\begin{array}{c}\text { [b] } \\
-- \\
-- \\
-- \\
-- \\
-- \\
-- \\
--\end{array}$ & $\begin{array}{r}1106 \\
1111 \\
1068 \\
983 \\
869 \\
725 \\
961 \\
1023 \\
1084\end{array}$ & $\begin{array}{l}561 \\
558 \\
558 \\
555 \\
548 \\
543 \\
508 \\
509 \\
517\end{array}$ & $\begin{array}{l}557 \\
557 \\
558 \\
554 \\
548 \\
543 \\
508 \\
510 \\
515\end{array}$ & $\begin{array}{l}559 \\
557 \\
557 \\
553 \\
546 \\
542 \\
506 \\
509 \\
516\end{array}$ & $\begin{array}{l}199 \\
302 \\
208{ }^{\circ} \\
205 \\
206 \\
208 \\
264 \\
277 \\
290\end{array}$ & $\begin{array}{l}10 \\
6.3 \\
6.9 \\
5.1 \\
3.3 \\
1.5 \\
3.9 \\
4.8 \\
6.9\end{array}$ \\
\hline \multirow[t]{2}{*}{$\begin{array}{l}\text { Pre- } \\
\text { condi- } \\
\text { tioning }\end{array}$} & $\begin{array}{l}1.0 \\
5.0 \\
1.0 \\
5.0 \\
1.0 \\
5.0\end{array}$ & $\begin{array}{r}4.8 \\
21.8 \\
4.7 \\
21.9 \\
4.5 \\
21.5\end{array}$ & $\begin{array}{r}975 \\
1732 \\
969 \\
1730 \\
970 \\
1732\end{array}$ & $\begin{array}{l}-- \\
-- \\
-- \\
-- \\
--\end{array}$ & $\begin{array}{l}=- \\
-- \\
-- \\
-- \\
--\end{array}$ & $\begin{array}{r}832 \\
1175 \\
832 \\
1210 \\
836 \\
1225\end{array}$ & $\begin{array}{l}513 \\
558 \\
540 \\
559 \\
543 \\
558\end{array}$ & $\begin{array}{l}511 \\
554 \\
538 \\
555 \\
541 \\
555\end{array}$ & $\begin{array}{l}541 \\
557 \\
538 \\
557 \\
543 \\
556\end{array}$ & $\begin{array}{l}550 \\
554 \\
554 \\
554 \\
548 \\
554\end{array}$ & $\begin{array}{l}1.0 \\
6.2 \\
0.9 \\
6.2 \\
.9 \\
6.1\end{array}$ \\
\hline & \multicolumn{9}{|c|}{$478 \mathrm{~K}$ Inlet Temperature } & & \\
\hline $\begin{array}{l}\text { Prior to } \\
\text { power } \\
\text { oscil- } \\
\text { lations }\end{array}$ & $\begin{array}{r}2.7 \\
6.0 \\
9.0 \\
11.6 \\
2.7 \\
6.0 \\
9.4 \\
12.3 \\
13.5\end{array}$ & $\begin{array}{l}12.0 \\
23.8 \\
35.8 \\
45.0 \\
11.8 \\
22.9 \\
35.1 \\
44.4 \\
47.8\end{array}$ & $\begin{array}{l}1320 \\
1589 \\
2021 \\
2191 \\
1278 \\
2014 \\
2212[\mathrm{c}] \\
2122[\mathrm{c}] \\
\text { [d] }\end{array}$ & $\begin{array}{l}-- \\
-- \\
-- \\
-- \\
-- \\
-- \\
--\end{array}$ & $\begin{array}{l}-- \\
-- \\
-- \\
-- \\
-- \\
-- \\
-- \\
--\end{array}$ & $\begin{array}{c}1087 \\
1173 \\
1284 \\
1569 \\
{[\mathrm{~b}][\mathrm{c}]} \\
1012[\mathrm{c}] \\
1390[\mathrm{c}] \\
{[\mathrm{d}]} \\
{[\mathrm{d}]}\end{array}$ & $\begin{array}{l}507 \\
523 \\
538 \\
538 \\
504 \\
516 \\
529 \\
539 \\
539 .\end{array}$ & $\begin{array}{l}508 \\
517 \\
526 \\
530 \\
503 \\
514 \\
524 \\
520 \\
538\end{array}$ & $\begin{array}{l}505 \\
521 \\
532 \\
531 \\
502 \\
513 \\
524 \\
533 \\
534\end{array}$ & $\begin{array}{l}529 \\
529 \\
538 \\
680 \\
567 \\
573 \\
567 \\
661 \\
674\end{array}$ & $\begin{array}{r}3.4 \\
7.3 \\
11.0 \\
11.0 \\
3.1 \\
6.4 \\
10.2 \\
11.1 \\
11.8\end{array}$ \\
\hline
\end{tabular}

[a] Not corrected for perturbation effects.

[b] Test Rod GC 504 did not contain off-center thermocouples in the 0 and $120^{\circ}$ locations.

[c] Questionable.

[d] Detector nor responding properily. 
$\underline{\text { TABLE XI }}$

PRELIMINARY POWER CALIBRATION DATA FROM TEST GC 2-1

\begin{tabular}{|c|c|c|c|c|c|}
\hline \multirow[b]{2}{*}{$\begin{array}{l}\text { Indicated } \\
\text { Core } \\
\text { Power } \\
\text { (MW) } \\
\end{array}$} & \multirow[b]{2}{*}{$\begin{array}{l}\text { SPND } 3 \\
\text { Current } \\
(n A)\end{array}$} & \multicolumn{4}{|c|}{ Test Rod Peak Power Density } \\
\hline & & $\begin{array}{r}\text { Rod } \\
\mathrm{GC} 501 \\
(\mathrm{~kW} / \mathrm{m}) \\
\end{array}$ & $\begin{array}{r}\text { Rod } \\
\mathrm{GC} 502 \\
(\mathrm{~kW} / \mathrm{m}) \\
\end{array}$ & $\begin{array}{r}\text { Rod } \\
\mathrm{GC} 503 \\
(\mathrm{~kW} / \mathrm{m}) \\
\end{array}$ & 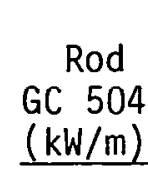 \\
\hline 0.5 & 4.64 & 2.4 & 2.92 & 2.4 & 2.55 \\
\hline 1.0 & 9.6 & 4.58 & 5.4 & 4.81 & 4.74 \\
\hline 1.5 & 13.76 & 6.95 & 8.08 & 7.3 & 6.99 \\
\hline 2.0 & 18.4 & 9.24 & 10.64 & 9.77 & 9.33 \\
\hline 2.65 & 25.6 & 12.75 & 14.2 & 9.32 & 12.26 \\
\hline 2.90 & 26.24 & 12.97 & 14.66 & 13.1 & 12.68 \\
\hline 5.0 & 45.12 & 21.7 & 25.22 & 21.75 & 21.85 \\
\hline 6.0 & 49.44 & 26.83 & 27.75 & 24.26 & 23.79 \\
\hline 9.0 & 74.56 & 41.1 & 41.5 & 35.2 & 35.83 \\
\hline 11.60 & 95.68 & 51.62 & 51.78 & 45.65 & 45.05 \\
\hline 13.5 & 102.1 & 52.11 & 56.79 & 48.85 & 47.76 \\
\hline
\end{tabular}

waveshape for the cladding surface temperature oscillations at the $240^{\circ}$ orientation on Rod GC 501 also indicated the initiation of waveshape changes. Figure 61 shows a comparison of the two waveshapes at a core power level of 12.3 MW. At this power level, Rod GC 501 had a peak power density of $48 \mathrm{~kW} / \mathrm{m}$, and Rod GC 504 had a peak power density of $44 \mathrm{~kW} / \mathrm{m}$. Figure 62 shows the Rod GC 501 cladding temperature oscillations in comparison with the reactor power oscillations as indicated by Supplemental Linear Power Chamber No. 1 (SLP1).

Following the above described observations at a power level of $12.3 \mathrm{MW}$, the core power was increased to $13.5 \mathrm{MW}$ to provide peak power densities of $48 \mathrm{~kW} / \mathrm{m}$ in Rod GC 504 and $52 \mathrm{~kW} / \mathrm{m}$ in Rod GC 501. Figures 63 through 66 show representative relative waveshapes obtained for the different azimuthal cladding temperature oscillation orientations on Rod GC 504 and for Rod GC 501 at $240^{\circ}$. As shown in Figure 64, at the increased power level the cladding temperature oscillations on Rod GC 504 at $0^{\circ}$ were changed significantly, while the cladding temperature oscillations at $240^{\circ}$ were barely beginning to show a waveshape change. Since the oscillations at $240^{\circ}$ did not show waveshape changes at 


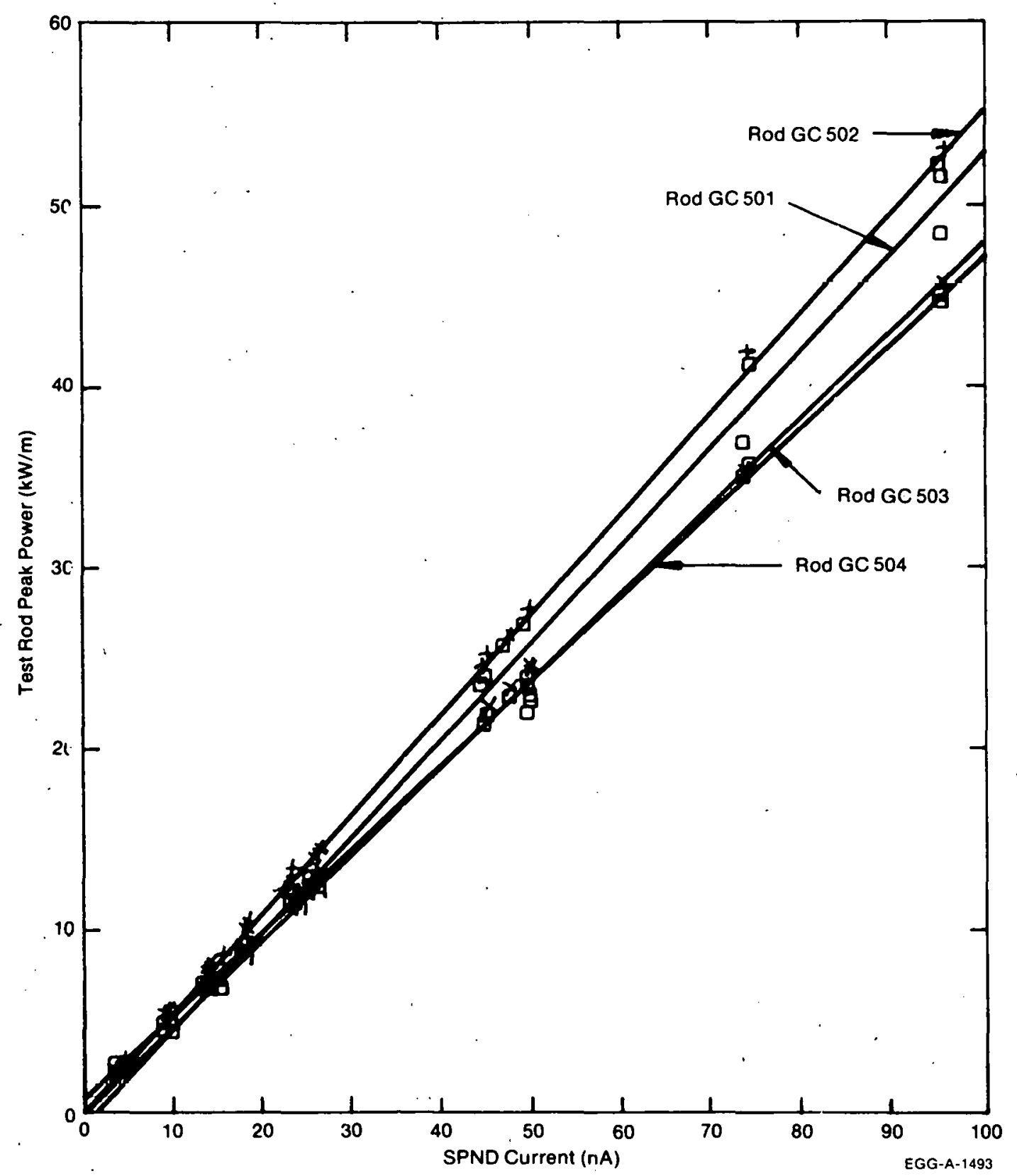

Fig. 58 Test fuel rod peak power density as a function of SPND 3 current for Test GC 2-1.

$44 \mathrm{~kW} / \mathrm{m}$, but did show changes at $48 \mathrm{~kW} / \mathrm{m}$, it appears that the power density threshold for waveshape changes may be at a nominal value somewhere between 44 and $48 \mathrm{~kW} / \mathrm{m}$ for an argon-filled rod with a $0.229-\mathrm{mm}$ initial gap width and $2.6-\mathrm{MPa}$ initial internal pressure.

The observed waveshape changes on the cladding surface temperature oscillations are not quite the same as predicted by FRAP-T2 for gap closure and opening (Figure 56) but may be indicative of this behavior. Posttest analyses may provide a better understanding of the meaning of the observed waveshape changes. 
TABLE XII

PRELIMINARY STEADY STATE GAP CONDUCTANCE VALUES FROM TEST GC 2-1

\begin{tabular}{|c|c|c|c|c|c|}
\hline $\begin{array}{l}\text { Test Rod Peak } \\
\text { Power Density } \\
(\mathrm{kW} / \mathrm{m})\end{array}$ & $\begin{array}{l}\text { Test Rod } \\
\text { No. } \\
\end{array}$ & $\begin{array}{c}\text { Fuel Centerl ine } \\
\text { Temperature } \\
(\mathrm{K})\end{array}$ & $\begin{array}{r}\text { Cladding } \\
\left(240^{\circ}\right. \\
\end{array}$ & $\begin{array}{l}\text { Temperature } \\
\text { orientation) } \\
(\mathrm{K})\end{array}$ & $\begin{array}{l}\text { Calculated } \\
\text { Gap Conductance } \\
\left(\mathrm{kW} / \mathrm{m}^{2}-\mathrm{k}\right) \\
\end{array}$ \\
\hline $\begin{array}{r}2.4 \\
4.8 \\
7.2 \\
13.0 \\
21.7 \\
26.8 \\
41.1\end{array}$ & $\begin{array}{ll}\text { GC } & 501 \\
\text { GC } & 501 \\
\text { GC } & 501 \\
\text { GC } & 501 \\
\text { GC } & 501 \\
\text { GC } & 501 \\
\text { GC } & 501\end{array}$ & $\begin{array}{l}946 \\
1135 \\
1351 \\
1582 \\
1879 \\
1837 \\
2071\end{array}$ & & $\begin{array}{l}542 \\
538 \\
506 \\
502 \\
557 \\
519 \\
530\end{array}$ & $\begin{array}{l}0.196 \\
0.279 \\
0.305 \\
0.516 \\
0.781 \\
1.092 \\
1.928\end{array}$ \\
\hline $\begin{array}{r}2.9 \\
5.6 \\
8.4 \\
14.5 \\
25.3\end{array}$ & $\begin{array}{ll}\text { GC } & 502 \\
\text { GC } & 502 \\
\text { GC } & 502 \\
\text { GC } & 502 \\
\text { GC } & 502\end{array}$ & $\begin{array}{r}599 \\
649 \\
681 \\
878 \\
1148\end{array}$ & & $\begin{array}{l}541 \\
540 \\
505 \\
556 \\
555\end{array}$ & $\begin{array}{l}3.32 \\
3.656 \\
3.172 \\
3.348 \\
2.685\end{array}$ \\
\hline $\begin{array}{r}2.4 \\
4.8 \\
7.3 \\
9.8 \\
13.2 \\
21.8 \\
35.5 \\
48.8\end{array}$ & $\begin{array}{ll}\text { GC } & 503 \\
\text { GC } & 503 \\
\text { GC } & 503 \\
\text { GC } & 503 \\
\text { GC } & 503 \\
\text { GC } & 503 \\
\text { GC } & 503 \\
\text { GC } & 503\end{array}$ & $\begin{array}{r}638 \\
710 \\
787 \\
863 \\
957 \\
1234 \\
1489 \\
1936\end{array}$ & . & $\begin{array}{l}542 \\
547 \\
552 \\
557 \\
557 \\
558 \\
527 \\
536\end{array}$ & $\begin{array}{l}1.047 \\
1.367 \\
1.541 \\
1.684 \\
1.864 \\
2.061 \\
3.221 \\
3.611\end{array}$ \\
\hline $\begin{array}{r}2.5 \\
4.7 \\
8.8 \\
12.7 \\
23.8 \\
35.1 \\
45.0\end{array}$ & $\begin{array}{ll}\text { GC } & 504 \\
\text { GC } & 504 \\
\text { GC } & 504 \\
\text { GC } & 504 \\
\text { GC } & 504 \\
\text { GC } & 504 \\
\text { GC } & 504\end{array}$ & $\begin{array}{r}794 \\
979 \\
1235 \\
1355 \\
1589 \\
2212 \\
2191\end{array}$ & . & $\begin{array}{l}542 \\
546 \\
509 \\
516 \\
521 \\
524 \\
531\end{array}$ & $\begin{array}{l}0.342 \\
0.390 \\
0.463 \\
0.632 \\
1.197 \\
1.191 \\
2.035\end{array}$ \\
\hline
\end{tabular}

1.5.4 Discussion and Conclusions. The objectives of Test GC 2-1 were met. Data were obtained from which on-line gap conductance values have been, and will continue to be, evaluated by both steady state and power oscillation methods. Data were also obtained to provide checks on reproducibility of test results.

On-line observations of cladding surface temperature oscillation waveshapes indicate that for the argon-filled rod, a threshold for gap closure may be in the range of 44 to $48 \mathrm{~kW} / \mathrm{m}$. For the xenon-filled rod, the threshold may be in the $48-$ to $52-\mathrm{kW} / \mathrm{m}$ range. The cladding surface temperature oscillations did not provide any on-line indications of the 
TABLE XIII

PRELIMINARY POWER OSCILLATION DATA FROM GAP CONDUCTANCE TEST GC 2-1, TEST ROD GC 501 (cladding thermocouple at $240^{\circ}, 0.452 \mathrm{~m}$ above bottom of fuel stack)

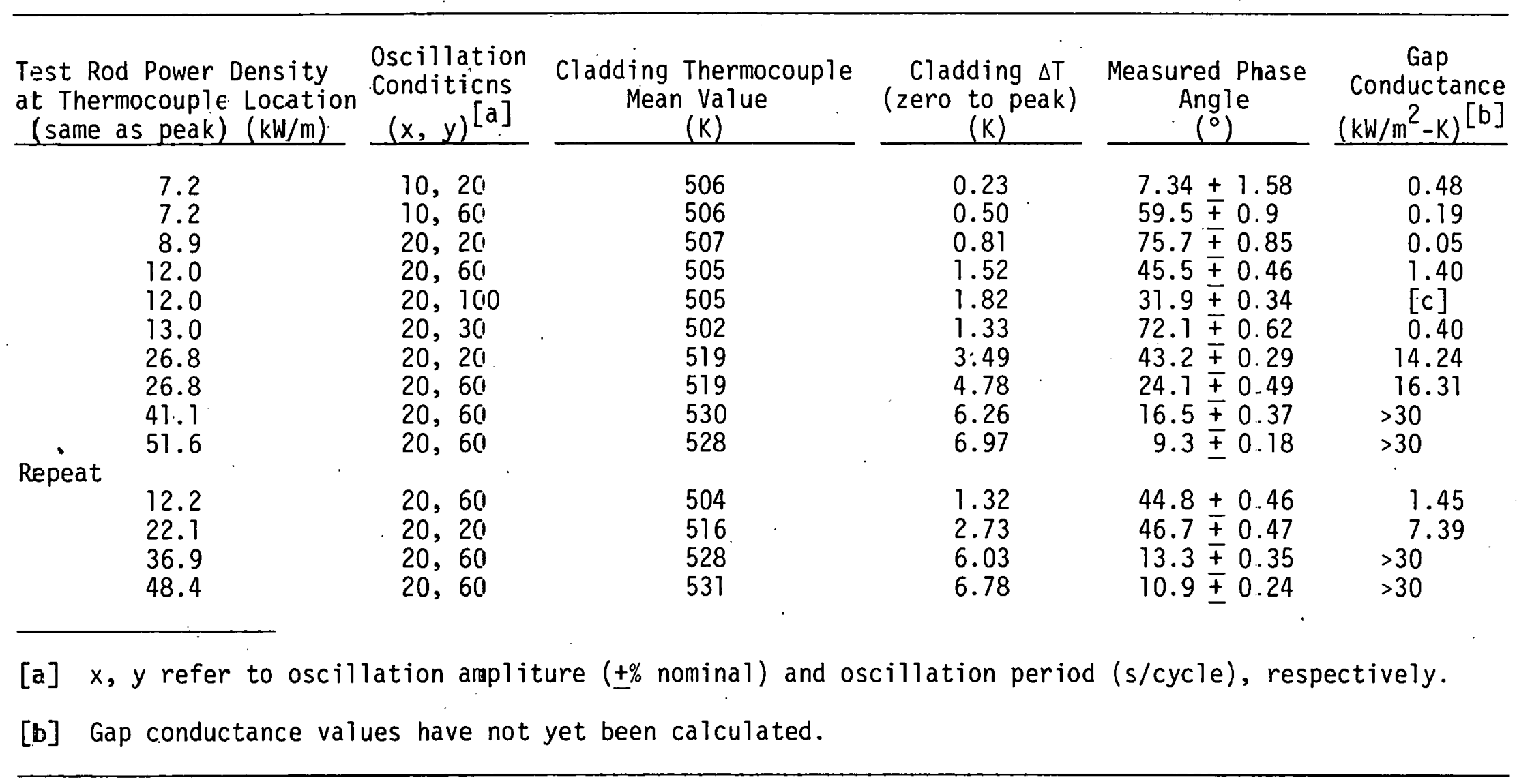


TAELE XIV

PRELIMINARY PONER OSCILLFTION DATA. FFOM EAP CONDUCTANCE TEST GC 2-1, TEST ROD GC 502 (cladding thermacouple at $240^{\circ}, 0.452 \mathrm{~m}$ above bottom of fuel stack)

\begin{tabular}{|c|c|c|c|c|c|}
\hline $\begin{array}{l}\text { Test Rod .Power Density } \\
\text { at Thermocouple Location } \\
\text { (same as peak) (kW'm) }\end{array}$ & $\begin{array}{l}\text { 0iciliation } \\
\text { Conditions } \\
i x, y]^{[a]} \\
\end{array}$ & $\begin{array}{c}\text { Clacdirig Thermocouple } \\
\text { Mean lialue } \\
\text { (k.) }\end{array}$ & $\begin{array}{c}\text { Cladding } \Delta \mathbf{T} \\
\text { (zero to peaki) } \\
(K)\end{array}$ & $\begin{array}{c}\text { Measured Phase } \\
\text { Angle } \\
\left({ }^{\circ}\right)\end{array}$ & $\begin{array}{c}\text { Gap } \\
\text { Conductance } \\
\left(\mathrm{kW} / \mathrm{m}^{2}-\mathrm{K}\right)^{[\mathrm{b}]} \\
\end{array}$ \\
\hline 13.6 & 20,20 & 505 & 1.24 & $44.6 \pm 0.55$ & 9.09 \\
\hline 27.8 & $-20,20$ & 516 & 2.43 & $41.5 \pm 0.27$ & 21.51 \\
\hline 41.5 & $20 ; 20$ & $5 \hat{c} 6$ & 3.34 & $40.4 \pm 0.34$ & 25.66 \\
\hline 51.8 & 20,20 & $5 \check{c} 8$ & 3.78 & $41.8 \pm 0.3$ & 16.40 \\
\hline
\end{tabular}

[a] $x, y$ refer to ascillatior amplitude ( $+\%$ nominal) and oscillation period (s/cycle), respectively. 


\section{$\underline{\text { TABLE XV }}$}

PRELIMINARY POWER OSCILLATION DATA FROM GAP CONDUCTANCE TEST GC 2-1, TEST ROD GC: 503 (cladding thermccouple at $240^{\circ}, 0.452 \mathrm{~m}$ above bottom of fuel stack)

\begin{tabular}{|c|c|c|c|c|c|c|}
\hline \multicolumn{2}{|c|}{$\begin{array}{l}\text { Test Rod Power Density } \\
\text { at Thermocouple Location } \\
\text { (same as peak) (kW/m) }\end{array}$} & $\begin{array}{l}\text { Oscillation } \\
\text { Conditicins } \\
(x, y)^{[a]} \\
\end{array}$ & $\begin{array}{c}\text { Cladding Thermocouple } \\
\text { Mean Value } \\
(\mathrm{K})\end{array}$ & $\begin{array}{r}\begin{array}{r}\text { Cladding } \Delta T \\
\text { (zero to peak) } \\
(\mathrm{K})\end{array} \\
\end{array}$ & $\begin{array}{c}\text { Measured Phase } \\
\text { Angle } \\
\left({ }^{\circ}\right)\end{array}$ & $\begin{array}{r}\text { Gap } \\
\text { Conductance } \\
\left(\mathrm{kW} / \mathrm{m}^{2}-\mathrm{k}\right)^{[\mathrm{b}]} \\
\end{array}$ \\
\hline \multirow[t]{2}{*}{ Repeat } & $\begin{array}{l}7.3 \\
7.3 \\
9.2 \\
12.5 \\
13.1 \\
24.3 \\
24.3 \\
35.2 \\
45.6 \\
45.6\end{array}$ & $\begin{array}{ll}10, & 20 \\
10, & 60 \\
20, & 20 \\
20, & 20 \\
20, & 20 \\
20, & 10 \\
20, & 30 \\
20, & 30 \\
20, & 10 \\
20, & 30\end{array}$ & $\begin{array}{l}506 \\
506 \\
508 \\
507 \\
515 \\
520 \\
520 \\
533 \\
535 \\
535\end{array}$ & $\begin{array}{l}0.68 \\
1.07 \\
1.46 \\
1.28 \\
3.57 \\
1.79 \\
3.77 \\
5.26 \\
3.31 \\
5.62\end{array}$ & $\begin{array}{l}56.8 \pm 0.53 \\
31.3 \pm 7.65 \\
52.6 \pm 0.26 \\
52.9 \pm 0.45 \\
49.9 \pm 0.25 \\
59.2 \pm 0.45 \\
40.7 \pm 0.27 \\
38.5 \pm 0.27 \\
51.7 \pm 0.28 \\
39.0 \pm 0.14\end{array}$ & $\begin{array}{r}2.70 \\
2.94 \\
2.74 \\
3.79 \\
4.97 \\
3.96 \\
7.49 \\
14.06 \\
7.58 \\
14.05\end{array}$ \\
\hline & $\begin{array}{l}12.6 \\
24.6 \\
35.5 \\
45.5\end{array}$ & $\begin{array}{l}20,20 \\
20,30 \\
20,30 \\
20,30\end{array}$ & $\begin{array}{l}505 \\
516 \\
527 \\
533\end{array}$ & $\begin{array}{l}1.04 \\
2.80 \\
4.31 \\
5.63\end{array}$ & $\begin{array}{l}48.6+0.31 \\
40.9+0.35 \\
40.6 \pm 0.26 \\
37.7 \pm 0.23\end{array}$ & $\begin{array}{r}5.71 \\
7.26 \\
8.82 \\
20.00\end{array}$ \\
\hline
\end{tabular}

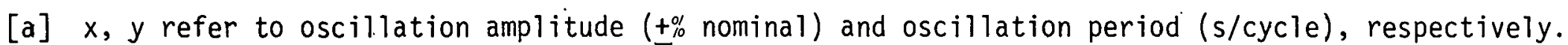


TAB_E XVI

PRELIMINARY FOWER OSCILLATION DATA FROM GAP CONDUCTANCE TEST GC ?-1, TEST ROD 504 (clacding thermocouples at $240^{\circ}, 0.452 \mathrm{~m}$ above bottom of fuel stack)

\begin{tabular}{|c|c|c|c|c|c|c|}
\hline $\begin{array}{l}\text { Test Rod Power Density } \\
\text { at Thermocouple Location } \\
\text { (same as peak) (kN/m) }\end{array}$ & $\begin{array}{l}\text { Osc }=11 \text { lation } \\
\text { Concitions } \\
(x: y)^{[a]}\end{array}$ & $\begin{array}{l}\text { Cladding Thermocouple } \\
\text { Orientatior } \\
\left(0^{\circ}\right)\end{array}$ & $\begin{array}{c}\text { Cladding Thermocouple } \\
\text { Mean Value } \\
(\mathrm{K})\end{array}$ & $\begin{array}{c}\text { Cladding } \Delta T \\
\text { (zero to peak: } \\
\text { (K) }\end{array}$ & $\begin{array}{c}\text { Measured Phase } \\
\text { Angle } \\
\left(0^{\circ}\right)\end{array}$ & $\begin{array}{c}\text { Gap } \\
\text { Conductance } \\
\left(\mathrm{kW} / \mathrm{m}^{2}-\mathrm{c}\right) \\
\end{array}$ \\
\hline 12.0 & 20,20 & 240 & 505 & 0.68 & $70.8 \pm 1.3$ & 0.64 \\
\hline 12.0 & 20,60 & 240 & 505 & 1.67 & $47.8 \pm 0.32$ & 1.25 \\
\hline 23.8 & 20,60 & 240 & 521 & 0.64 & $27.8 \pm 0.3$ & 6.89 \\
\hline 35.8 & 20,60 & 240 & 532 & 5.90 & $23.0 \pm 0.3$ & 28.05 \\
\hline 35.8 & 20,20 & 240 & 532 & 4.73 & $38.2 \pm 0.22$ & 34.78 \\
\hline 45.0 . & 20,60 & 240 & 531 & 7.18 & $19.4 \pm 0.2$ & 25.37 \\
\hline 47.8 & 15,60 & 0 & 534 & 3.05 & $16.2 \pm 0.74$ & $>30$ \\
\hline 47.8 & 15,20 & 0 & 534 & 1.72 & $28.9 \pm 1.3$ & $>30$ \\
\hline 47.8 & $1 E, 20$ & $i 20$ & 534 & 4.22 & $40.2 \pm 0.48$ & 25.00 \\
\hline 47.8 & $1 E, 20$ & 240 & 534 & 4.68 & $27.7 \pm 0.34$ & $>30$ \\
\hline
\end{tabular}

[a] $x, y$ refer to oscillation anplitude ( $+\%$ nominal) and oscillation period (s/cycle), respectively. 


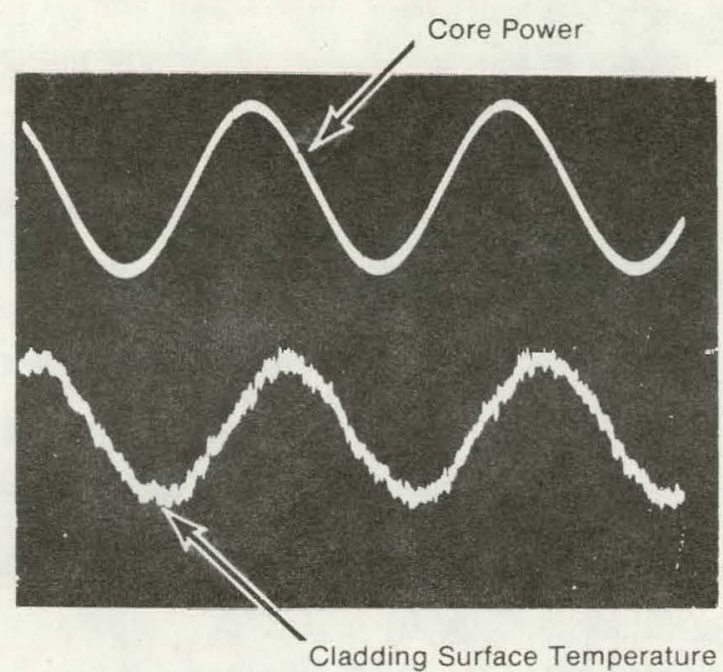

Fig. 59 Representative comparison of Rod GC 503 core power oscillations with cladding surface temperature oscillations at $240^{\circ}$ orientation with $\pm 20 \%$ amplitude, 20 s/cycle oscillations and $2.7 \mathrm{MW}$ core power.

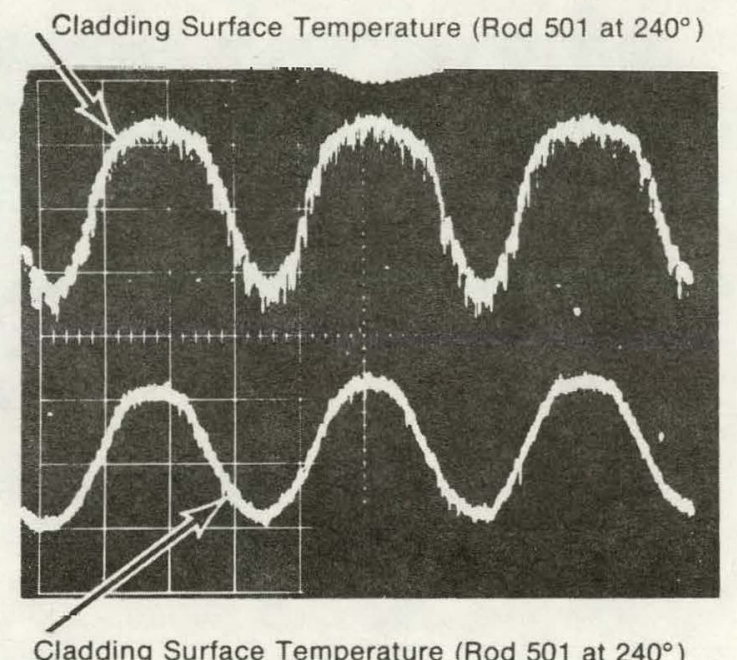

Fig. 61 Comparison of Rod GC 504 cladding surface temperature oscillations at $0^{\circ}$ orientation at power density of $44 \mathrm{~kW} / \mathrm{m}$ with Rod GC 501 cladding surface temperature oscillations at $240^{\circ}$ orientation at power density of $48 \mathrm{~kW} / \mathrm{m}$ with $\pm 20 \%$ amplitude, 60 s/cycle oscillations.

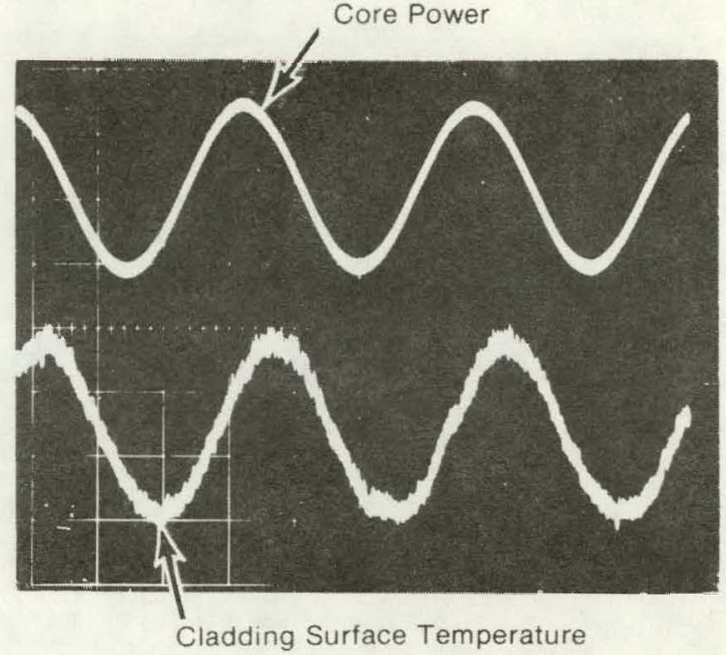

Fig. 60 Representative comparison of Rod GC 504 core power oscillations with cladding surface temperature oscillations at $240^{\circ}$ orientation with $\pm 20 \%$ amplitude, $260 \mathrm{~s} /$ cycle oscillations and $2.7 \mathrm{MW}$ core power.

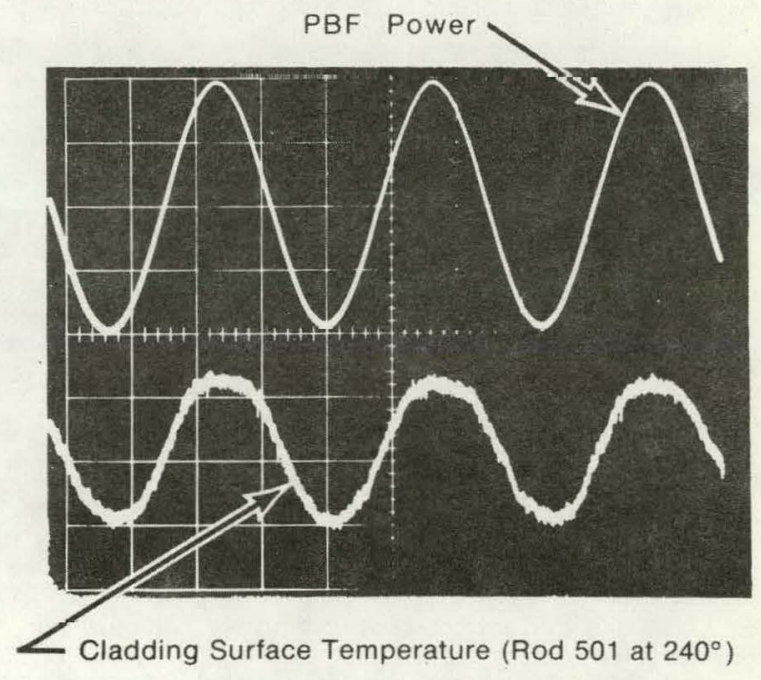

Fig. 62 Comparison of Rod GC 501 cladding temperature oscillations at $240^{\circ}$ orientation at power density of $48 \mathrm{~kW} / \mathrm{m}$ with PBF power oscillations with $\pm 20 \%$ amplitude, 60 s/cycle oscillations.

effects of gap closure for the two helium-filled rods. Detailed analyses of the tape data may provide more insight into the gap closure question.

The xenon-filled rod provided significantly higher fuel temperature measurements than the other rods. This was expected, although the magnitudes of the indicated temperatures were higher than expected. Figures 67 through 70 provide on-line measurements of the fuel centerline temperature as a function of test rod power density for each test rod. Although it was expected that hysteresis effects would be evident as a result of fuel 


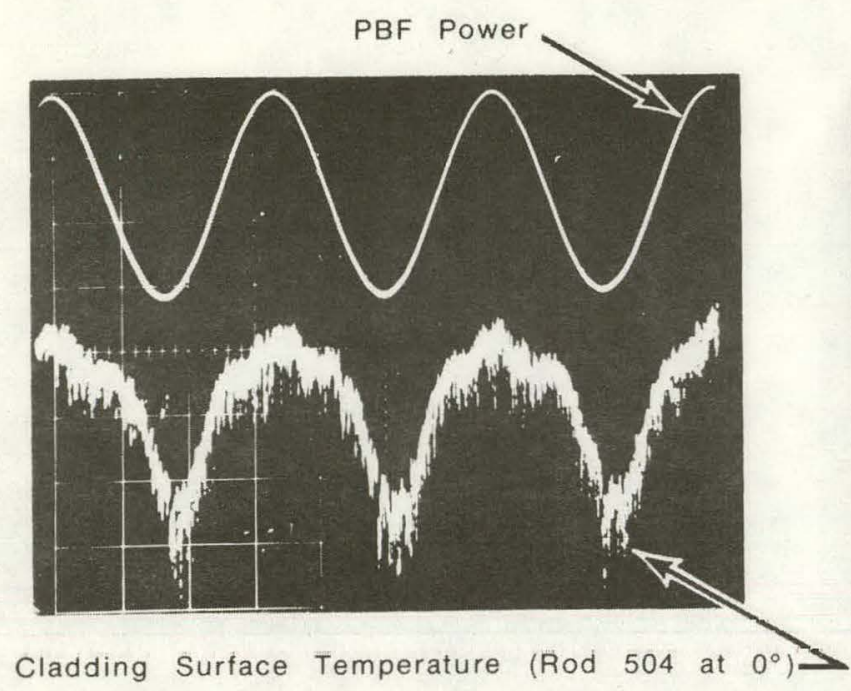

Fig. 63 Comparison of Rod GC 504 cladding temperature oscillations at $0^{\circ}$ orientation at power density of $48 \mathrm{~kW} / \mathrm{m}$ with PBF power oscillations with $\pm 15 \%$ amplitude, 60 s/cycle oscillations.

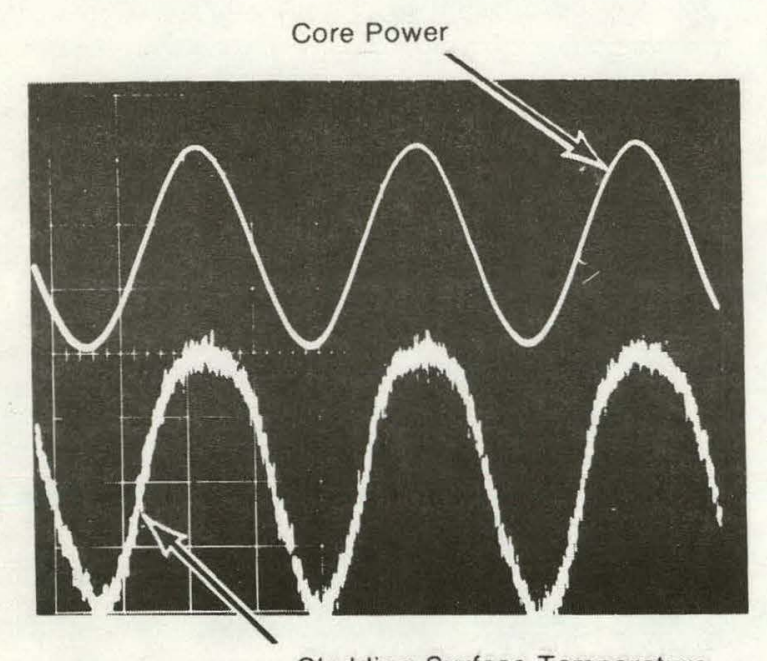

Cladding Surface Temperature

Fig. 65 Comparison of Rod GC 501 cladding temperature oscillations at $240^{\circ}$ orientation at power density of $52 \mathrm{~kW} / \mathrm{m}$ with core power oscillations with $\pm 15 \%$ amplitude, 60 s/cycle oscillations.

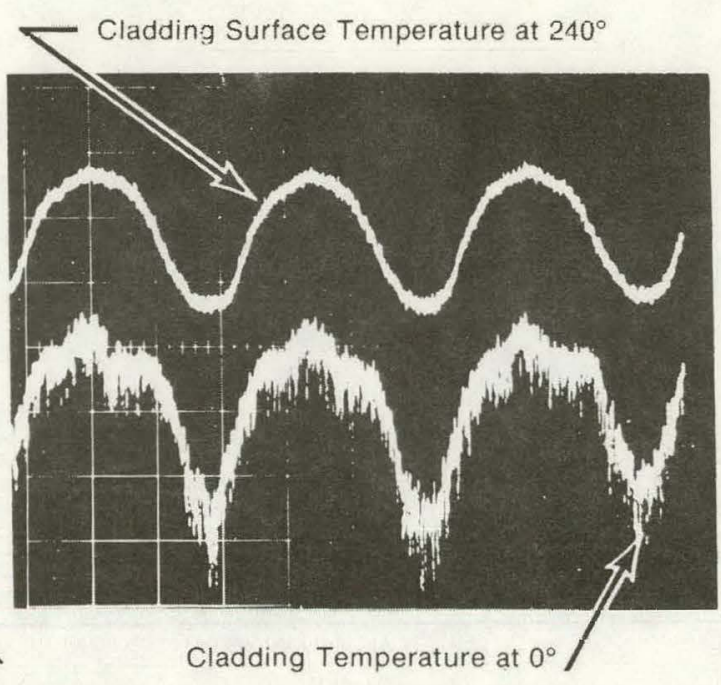

Fig. 64 Comparison of Rod GC 504 cladding temperature oscillations at 0 and $240^{\circ}$ orientation at power density of $48 \mathrm{~kW} / \mathrm{m}$ with $\pm 15 \%$ amplitude, $60 \mathrm{~s} /$ cycle oscillations.

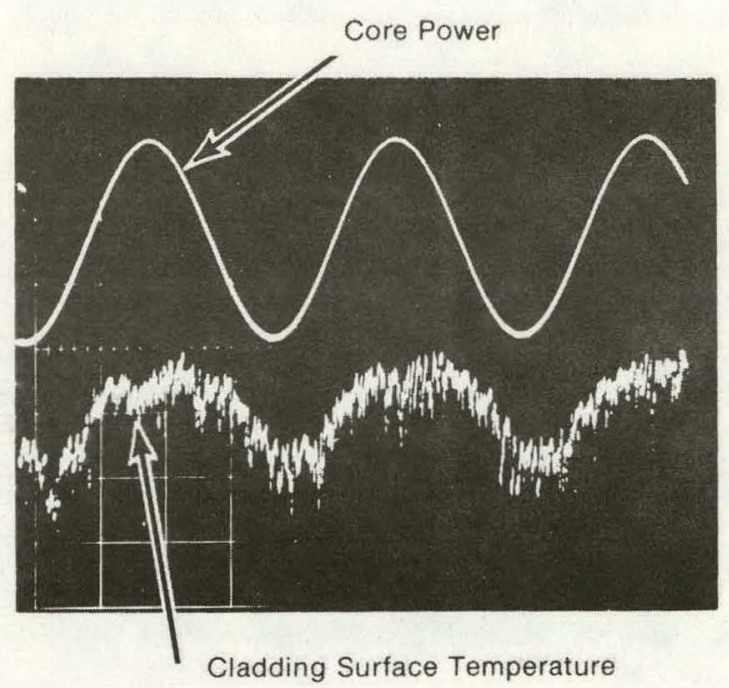

Fig. 66 Comparison of Rod GC 504 cladding temperature oscillations at $0^{\circ}$ orientation at power density of $48 \mathrm{~kW} / \mathrm{m}$ with core power oscillations with $\pm 15 \%$ amplitude, $20 \mathrm{~s} /$ cycle oscillations.

cracking with power cycling, none of the on-line temperature measurements indicated a significant hysteresis effect.

Comparison between the preliminary values obtained for steady state and power oscillation gap conductances for each rod with calculated values (FRAP-T2, cracked-pellet gap conductance model) are shown in Figures 71 through 74 . The steady state values compare very well with the cracked-pellet gap conductance model calculations, except for Rod GC 502, the helium-filled rod with a $0.1-\mathrm{mm}$ initial diametral gap. For this rod, the predicted gap conductance values are significantly higher than the measured steady state values and are generally higher than the power oscillation values. Also, for this rod, the 


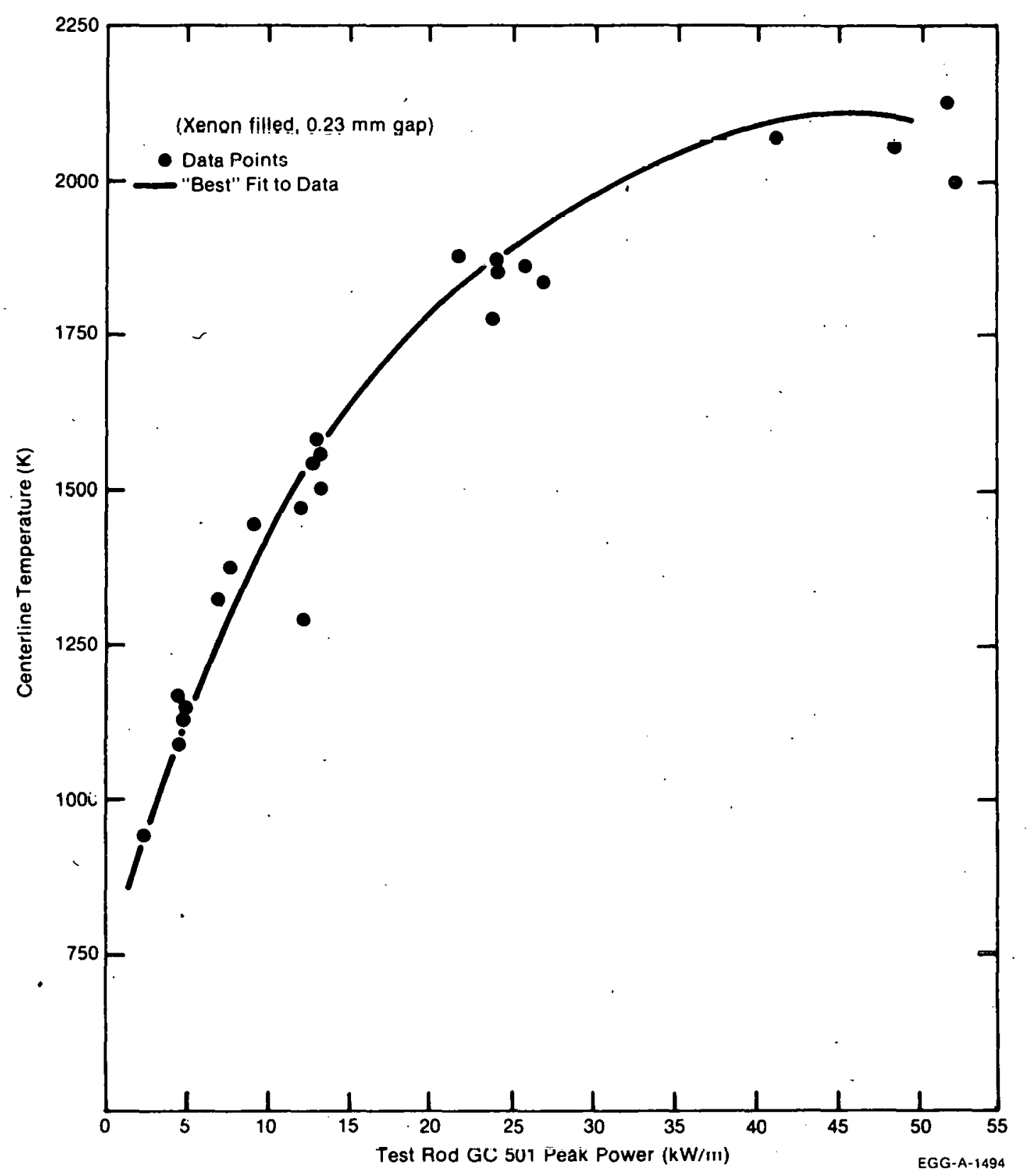

Fig. 67 Fuel centerline temperature as a function of test rod power density for Rod GC 501 during Test GC 2:1. 


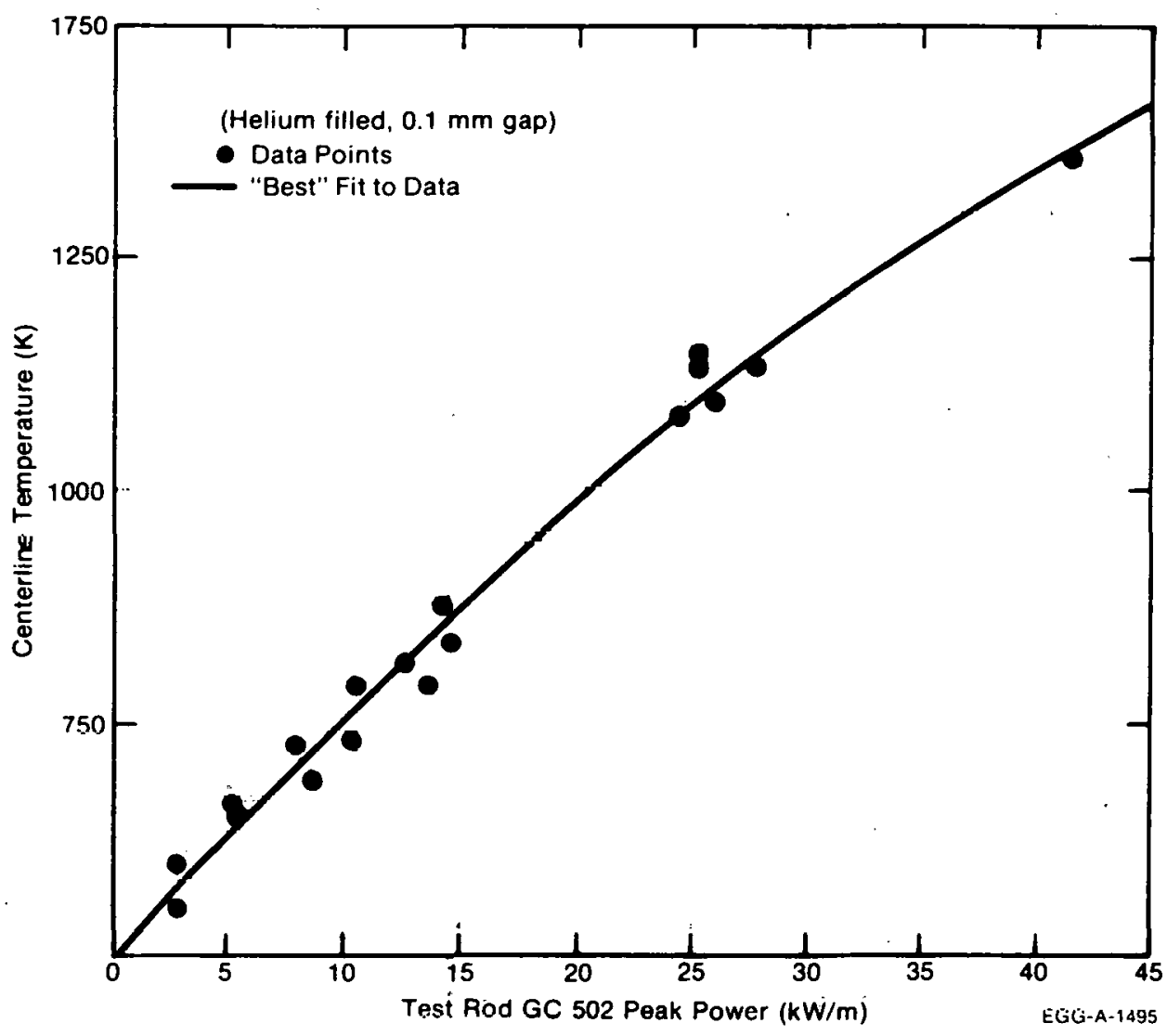

Fig. 68 Fuel centerline temperature as a function of test rod power density for Rod GC 502 during Test GC 2-1.

predictions indicate gap closure at a relatively low $(<31 \mathrm{~kW} / \mathrm{m})$ power density, whereas calculations for the other three test rods do not indicate gap closure until power densities greater than $50 \mathrm{~kW} / \mathrm{m}$ are reached.

Generally, differences between the steady state and power oscillation gap conductance values are large. The same trend for comparison between steady state, power oscillation, and calculated gap conductance values was observed for Test GC 1-3[18]. Similar comparisons of data obtained for PWR-design rods from the "piggyback" tests (for example, see Reference 19) have shown much better agreement between steady state and power oscillation gap conductance values. Also, the PWR rod experimental gap conductance values have consistently shown much better agreement with predictions based on the Ross and Stoute gap conductance model than with predictions based on the cracked-pellet model.

Figure 73 also shows comparisons of steady state data obtained from Test GC 2-1 with data obtained from Test GC i-3 for a similar BWR-design rod (helium-filled, 0.23-mm initial diametral gap). The comparisons show excellent agreement.

Other than basic differences between PWR and BWR rod designs, the only difference between Tests GC 1-3 and GC 2-1 and the piggyback tests are that Tests GC 1-3 and GC 2-1 have employed off-center fuel temperature measurements. In both of these tests, the 


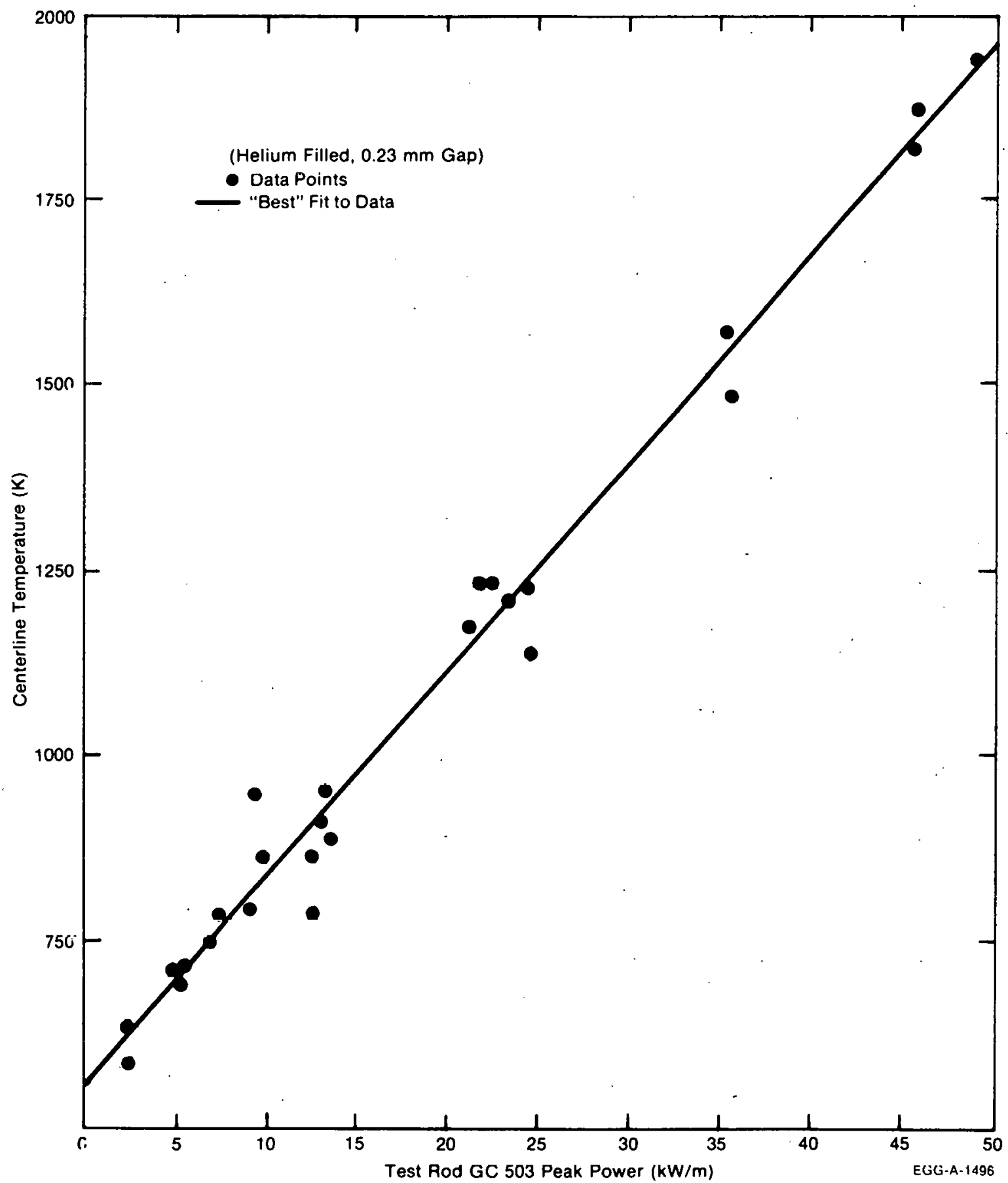

Fig. 69 Fuel centerline temperature as a function of test rod power density for Rod GC 503 during Test GC 2-1. 


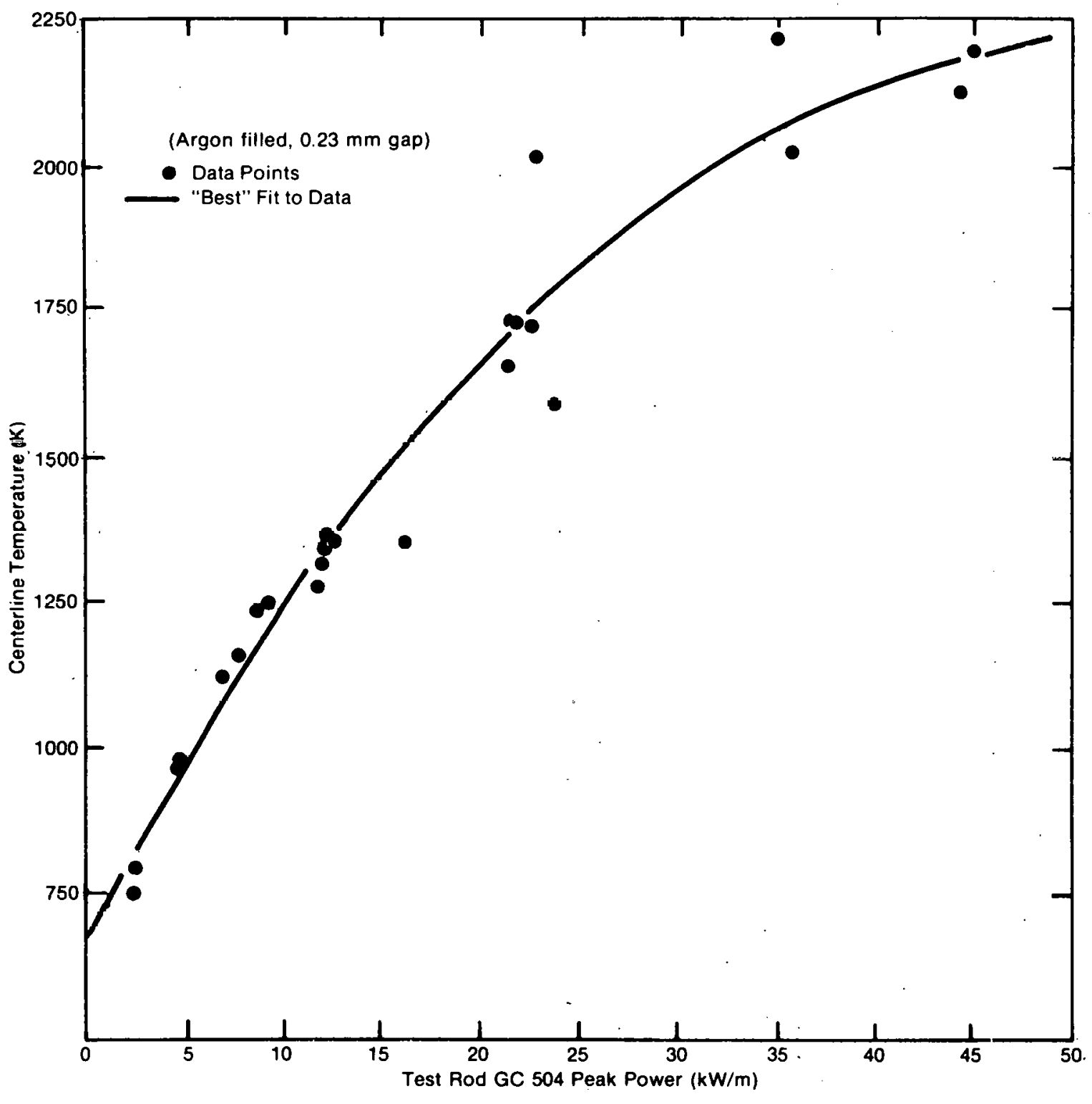

INEL-A-1509

Fig. 70 Fuel centerline temperature as a function of test rod power density for Rod GC 504 during Test GC 2-1.

off-center thermocouples have been located at the same axial level and azimuthal orientation as the cladding surface thermocouples. It is suspected that the off-center thermocouples may be influencing the phase relationships for the cladding surface thermocouples. For Test GC 2-2, the cladding thermocouples will be displaced azimuthally approximately $60^{\circ}$ in an attempt to avoid any influence from the off-center internal thermocouples. 


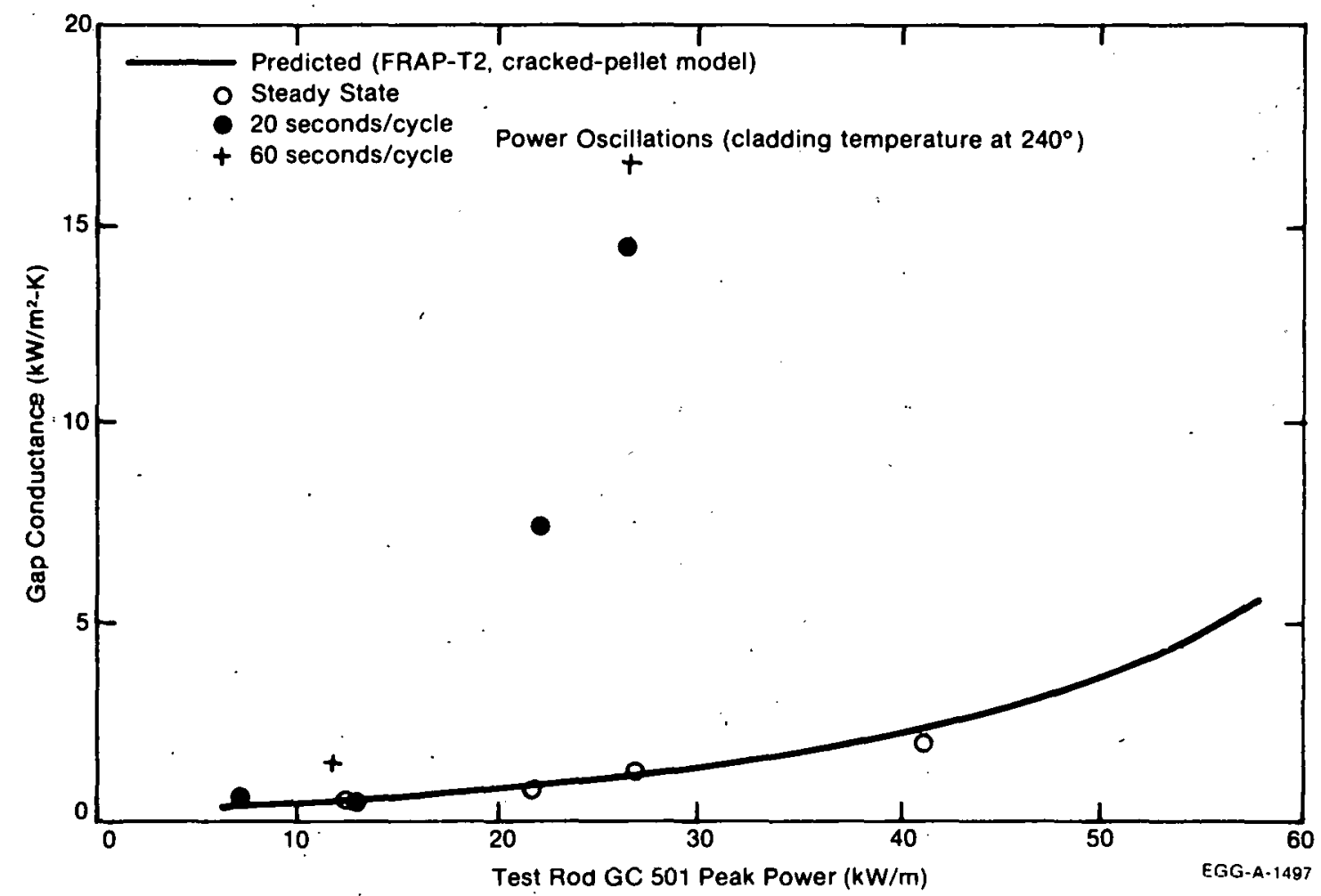

Fig. 71 Comparison of measured and predicted gap conductance values for Rod GC 501 during Test GC 2-1.

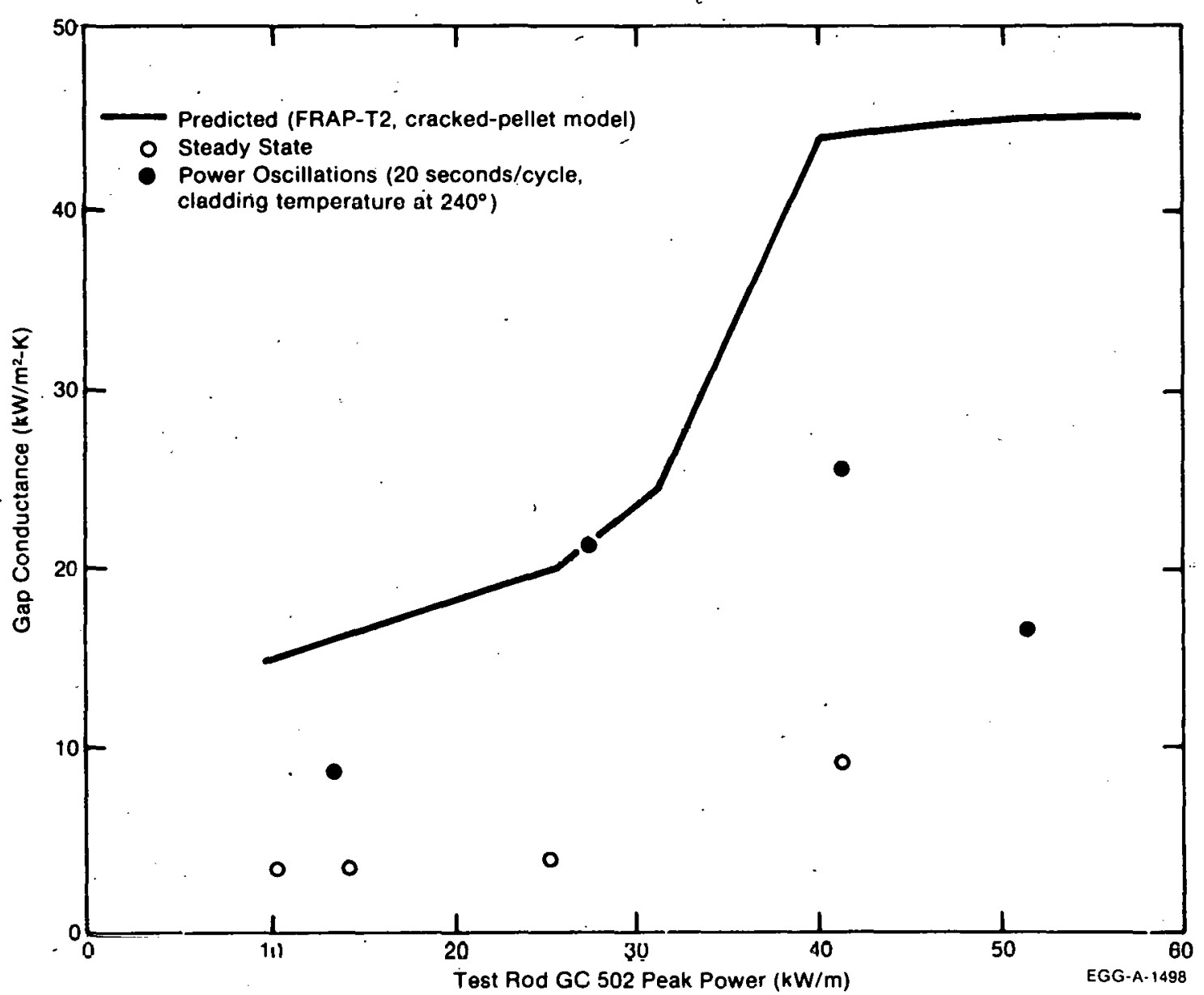

Fig. 72 Comparison of measured and predicted gap conductance values for Rod GC 502 during Test GC 2-1. 


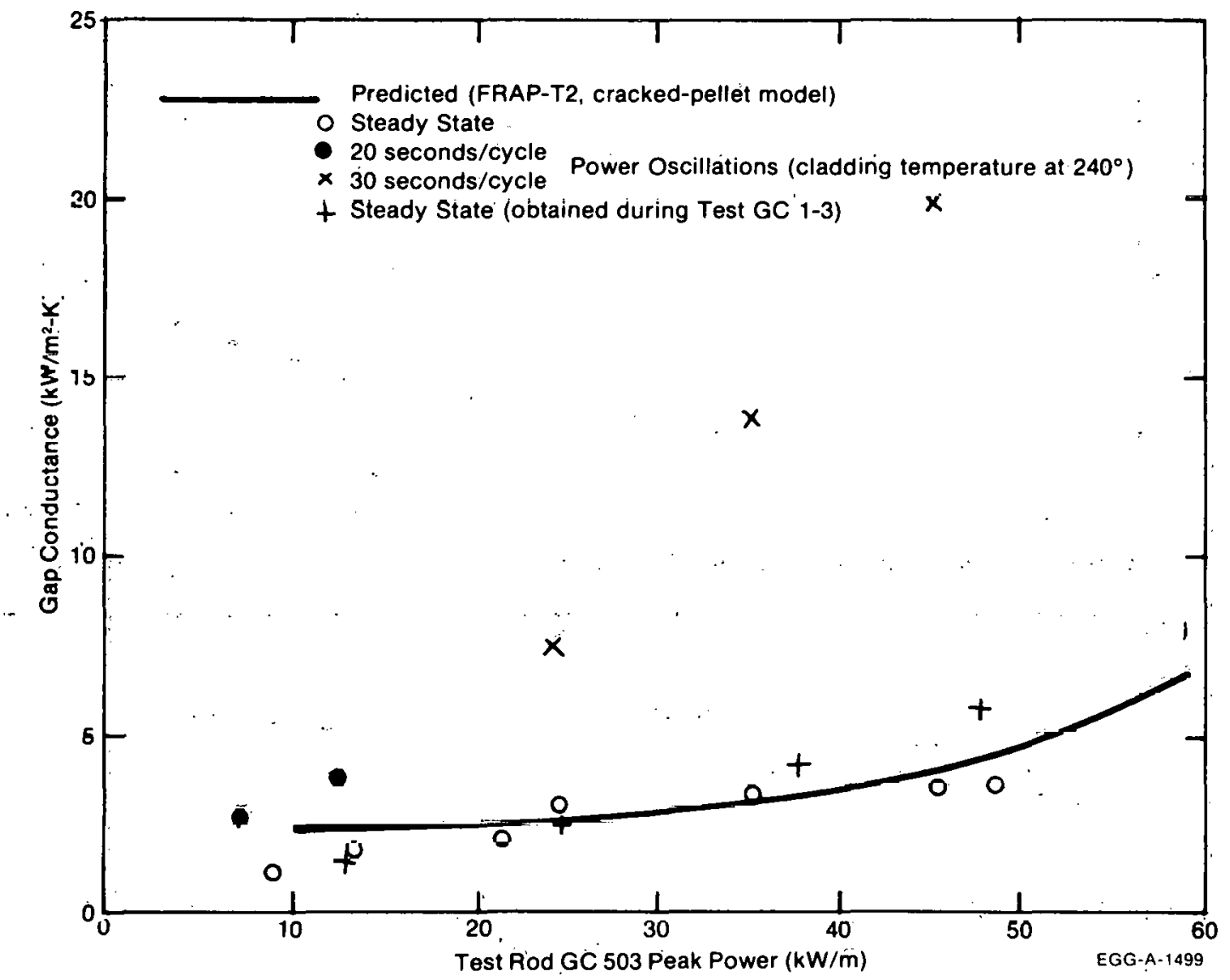

Fig. 73 Comparison of measured and predicted gap conductance values for Rod GC 503 during Test GC 2-1. and GC 1-3. 


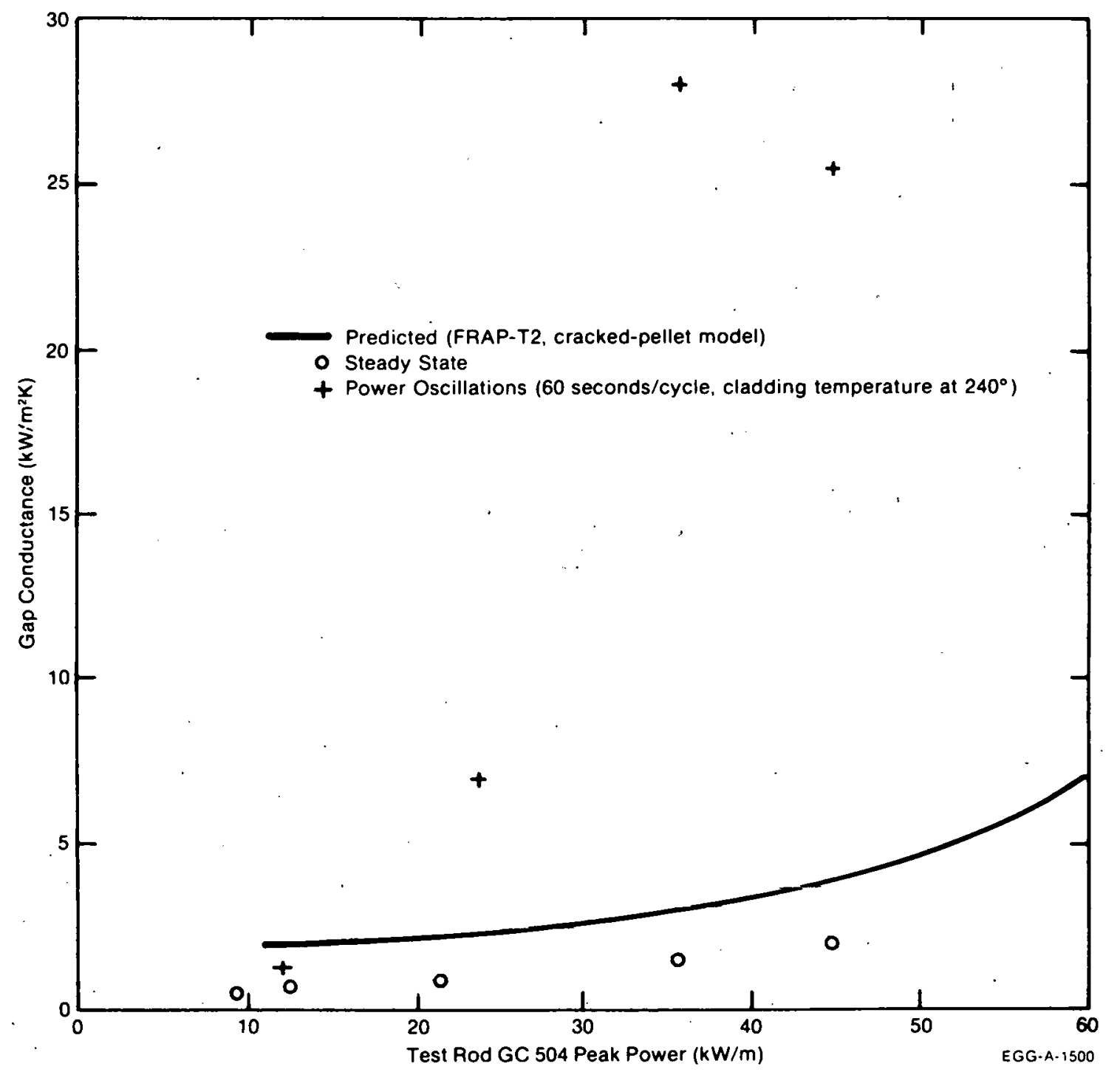

Fig. 74 Comparison of measured and predicted gap conductance values for Rod GC 504 during Test GC 2-1. 


\title{
2. THERMAL FUELS BEHAVIOR PROGRAM DEVELOPMENT AND EVALUATION
}

\author{
P. E. MacDonald and L. B. Thompson
}

Efforts were concentrated in the areas of program development, Halden fuel behavior research, light water reactor materials properties correlations, and postirradiation examination of power reactor fuel. The scope and objectives of these efforts are described in Reference 20.

This section contains a summary of the various program development and evaluation activities (Section 2.1), a zircaloy local strain at rupture model (Section 2.2), a zircaloy cladding creep model (Section 2.3), and additional results from axial gas flow experiments utilizing PWR fuel rods (Section 2.4).

\subsection{Summary of Ongoing Efforts}

Integration with foreign experiment programs proceeded in the areas of reactivity initiated accident (RIA) and loss-of-coolant accident (LOCA) research. RIA research collaboration was carried out in meetings at the Japan Atomic Energy Research Institute (JAERI) in Tokai to review the Nuclear Safety Research Reactor (NSRR) and PBF experiment progress and plans.

Technical liason with the Italian Government was initiated with discussion in Ispra and Rome concerning use of the ESSOR reactor ${ }^{[a]}$ for LOCA testing with bundled rods. In concert with this cooperation, an evaluation of the multinational COFAS ${ }^{[b]}$ proposal for an ESSOR loop capable of testing 52-rod bundles was performed in cooperation with Brookhaven National Laboratory. The COFAS test facility would simulate accidents of the LOCA, PCM, ATWS (anticipated transient without scram), and flow blockage type beginning in about 1983 if continued approvals are obtained.

Integration with German fuel behavior research work involved German - U.S. code comparisons and a return visit to Germany of the Karlsruhe representative to Idaho National Engineering Laboratory (INEL) for discussion of current programs. Early comparisons of the German SSYST and INEL FRAP-T code predictions for LOCA fuel behavior were presented at both a Norwegian conference and the Water Reactor Safety Research Information Meeting. This comparison work continues in an effort to improve both codes.

Comparisons were made of best estimate (BE) and evaluation model (EM) predictions for fuel behavior during a hypothetical LOCA in a typical $15 \times 15$ PWR plant. A best

[a] A test reactor located at the Ispra Research Center in northern Italy.

[b] A loop in the ESSOR reactor. 
estimate prediction of the behavior of a hot pin was provided by FRAP-T2 MOD-2, Version 7A BE, using initial conditions given by a FRAP-S1 MOD-1, Version 002, steady state calculation and thermal-hydraulic boundary conditions from the RELAP4/MOD-E(73) code with $\mathrm{BE}$ update from BE/EM study. Best estimate assumptions for both plant behavior and models were used. The evaluation model calculation was obtained from RELAP4/MOD-E(73) with EM update from BE/EM study (containing the conservative REFRAP fuel model) and by TOODEE-2. The results of the evaluation model were more conservative in the case of cladding surface and fuel centerline temperatures and indicated cladding ballooning, whereas no ballooning was predicted by the best estimate calculation.

Irradiation of the IFA-429 helium sorption and transient fission gas release experiment continued in the Halden Heavy Boiling Water Reactor in Halden, Norway. This experiment has undergone three complete irradiation cycles and has accumulated burnup of approximately $7000 \mathrm{MWd} / \mathrm{t}$.

Characterization of the IFA-429 in-core instrumentation has been completed. The characterizations include instrument function, location, materials, operating principles, calibration, possible decalibration, and measurement uncertainties.

Three fuel rods previously removed from IFA -429 have been prepared for shipment to England where they will undergo postirradiation examination. The postirradiation examination will include a determination of the quantity of helium absorbed by the fuel pellets in addition to routine gas sampling and metallographic examination.

The postirradiation examination of the IFA-226 mixed oxide test assembly is nearing completion in Denmark. The results from the postirradiation examination of these-high burnup fuel rods will be combined with the in-pile test data and with the results of physics calculations currently being performed to produce data on fuel thermal behavior and fission gas release.

The Halden data system, developed by EG\&G Idaho, Inc., has been refined and simplified for easier use. This system is presently being used to store, manipulate, and plot test data from IFA-226 and IFA-429. The data system will also handle the IFA-430 test data when it becomes available.

Models for cladding creep-down and surface emissivity were revised to include uncertainty estimates and to include consideration of data which were not available for the original models. The assignment of uncertainty limits to the model for cladding creep-down is very tentative because available biaxial in-reactor data were limited. However, significant new data allowed generalization of the model for cladding. surface emmissivity to consider surfaces with both thick and thin oxide layers. The effects of crud on the emissivity of the outer cladding surface were also considered.

A new model which incorporates the effects of fuel densification on fuel swelling was completed. The model calculates the combined effects of densification and swelling on 
in-reactor fuel dimensional changes using previously developed models for $\mathrm{UO}_{2}$ densification and swelling.

Local cladding strain components at rupture were modeled using preliminary data from cross sections of tubes burst in isothermal tests. This model is intended for use as a failure criterion with analytical models which predict local strain as a function of lemperature.

Section 2.2 and 2.3 describe the models for cladding creep-down and local strains at rupture.

The power reactor postirradiation examination program continues in several areas. Two BWR bundles from Peach Bottom 2 have been identified hy visual, ultrasonic, and eddy current testing as containing pellet-cladding interaction fuel failures. These will arrive at the INEL in March $1 Y^{\prime} / \mathrm{l} /$ to begin postirradiation examination.

A gamma scanner to accommodate full length commercial fuel rods is being designed. Final design of the pulsed eddy current scanner for commercial rods has been completed, and the package is out for bid.

The axial gas flow experiments using irradiated PWR fuel rods have been completed. Analyses are being performed to compare the effective gaps measured metallographically with those calculated from measured gas flow rates. The results will be used to improve the gas flow models employed in various fuel performance codes.

Equipment preparation is underway for the out-of-pile $\mathrm{UO}_{2}$-zircaloy reaction rate experiments. 'The experiments will assess the influence of time, temperature, fuel stoichiometry, and simulated fission products on $\mathrm{UO}_{2}-\mathrm{Zr}$ interaction. The high-pressure $\mathrm{UO}_{2}-\mathrm{Zr}$ interaction capsule has been designed and capsule materials have been ordered. $\mathrm{A}$ noble metal furnace is being built to perform the higher temperature experiments. The results will contribute to the understanding of the fuel-cladding interaction observed in power-cooling-mismatch experiments in PBF.

\subsection{Clädding Creep Rate}

\section{G. A. Reymann}

A correlation which describes the creep-down behavior of zircaloy cladding used in light water reactors (LWRs) is presented. The correlation is primarily based on data taken under actual PWR operating conditions.

2.2. I Introduction. The correlation developed here will be used in fuel performance computer models which determine fuel temperatures, fuel and cladding deformations, pellet-cladding mechanical interaction, and other irradiation-induced behavior mechanisms which may occur during normal (steady state) operation. 
The following four factors are used in the correlation describing zircaloy cladding creep rate: (a) temperature, (b) fast neutron flux $(>1 \mathrm{MeV})$, (c) time at temperature, and (d) circumferential stress. The model has been developed for the in-pile diametral creep of zircaloy -2 or zircaloy 4 cladding under a biaxial stress. The model is not appropriate for, and does not predict, the results of out-of-pile tests, uniaxial tests, or tests conducted under irradiation conditions significantly different from LWR conditions.

2.2.2 Analy tical Model Derivation. While there is an impressive amount of creep data available, only a very few are chosen for consideration here. Many of the data conflict, and it is likely that this conflict is the result of differing experiment conditions, differing material geometries, or actually different materials. To obtain a correlation which predicts creep under typical LWR conditions, data from other reactor systems and fast flux spectrums were excluded, along with out-of-pile data, uniaxial creep data, plate specimen data, zirconium-based alloy data other than zircaloy- 2 or zircaloy-4, and data from specimens with significantly different texture.

Only a few data were found to meet these extensive criteria. The best of these were from the Saxton Plutonium Project $[21,22,23]$, where many profilometry data are reported for different rods at varying axial locations and burnups. The rods were separated into groups of approximately the same burnup, and the cladding outside diameters were measured at various axial locations after $9750 \mathrm{~h}$ of operating time. The data pertaining to these rods are presented in Table XVII. Because there are only five data in this set, it was possible to fit them very well with a single correlation. However, a paper by Ibrahim ${ }^{\text {[24] }}$ contains data which satisfy all the criteria except the last, having been taken from material with a texture similar to pressure tube material. These data are presented in Table XVIII. Nevertheless, these Ibrahim data were tried in the correlation developed for cladding, largely because they cover somewhat different temperature and stress ranges. It was found that for operating times comparable to those of the Saxton data, the correlation predicted these pressure tube creep rates with reasonable accuracy if multiplied by a constant factor of 0.8 .

(1) Time Dependence. The time dependence of the correlation presented is of the same form as that proposed by Ibrahim ${ }^{[24]}$ :

$$
\varepsilon=\beta t^{m}
$$

where $\beta$ is constant if all other experiment perameters are held constant and only time allowed to vary, $t$ is the time, and $m$ is a constant. Creep with this strain-time relationship has been reported often in the literature for many different types of alloys ${ }^{[25]}$. It is usually described as the result of work hardening dominating over recovery. Ibrahim ${ }^{[24]}$ discusses this at some length in his paper. In a fast flux environment, Ibrahim finds that the best value for $m$ is 0.468 . Ross-Ross and Fidleris ${ }^{[26]}$ find an exponent ranging from 0.8 to 1 from their uniaxial data, while Fidleris ${ }^{[27]}$ estimates values from 0 to 0.33 . When Equation (3) is differentiated with respect to time to obtain $\dot{\epsilon}$, the time exponent is (m-1) which, in all the cases quoted above, will be either negative or zero. A negative value for $m$ indicates a creep rate continuously diminishing with time, and an adequate equation was developed using $\mathrm{m}=0.5$, which means that $\dot{\epsilon} \propto 1 / \sqrt{\mathrm{t}}$. 
TABLE XVII

SAXTON RODS CREEP DATA AT 9750 HOURS $^{[\mathrm{a}]}$

\begin{tabular}{|c|c|c|c|c|}
\hline $\begin{array}{l}\text { Rod Avg } \\
\text { Power } \\
(\mathrm{kW} / \mathrm{ft})\end{array}$ & $\begin{array}{l}\text { Initial } \\
\text { Backfill } \\
\text { Pressure } \\
\quad(\mathrm{Pa}) \\
\end{array}$ & $\begin{array}{c}\text { Average } \\
\text { Cladding } \\
\text { Stress } \\
\text { (MPa) } \\
\end{array}$ & $\begin{array}{c}\text { Average } \\
\text { Cladding } \\
\text { Temperature } \\
\text { (K) } \\
\end{array}$ & $\begin{array}{l}\text { Measured } \\
\text { Creep Rate } \\
(\mathrm{m} / \mathrm{m} \cdot \mathrm{h})\end{array}$ \\
\hline 4.74 & $1.034 \times 10^{5}$ & 89.7 & 614 & $1.31 \times 10^{-7}$ \\
\hline 5.21 & $1.034 \times 10^{5}$ & 89.0 & $6 ? .1$ & $1.44 \times 10^{-7}$ \\
\hline 5.64 & $1.034 \times 10^{5}$ & $88 . \dot{3}$ & 627 & $1.47 \times 10^{-7}$ \\
\hline .6 .50 & $1.034 \times 10^{5}$ & 86.9 & 629 & $1.31 \times 10^{-7}$ \\
\hline 7.90 & $1.034 \times 10^{5}$ & 83.5 & 633 & $5.92 \times 10^{-8}$ \\
\hline
\end{tabular}

[a] A11 rods were in a fast flux (>1 MeV) of $2.168 \times 10^{17} \mathrm{n} / \mathrm{m}^{2} \cdot \mathrm{s}$.

TABLE XVIII

IBRAHIM PRESSURE TUBE CREEP DATA AT 10,000 HOURS [a]

\begin{tabular}{|c|c|c|}
\hline \multicolumn{2}{|c|}{$\begin{array}{c}\text { Average Cladding Stress } \\
(\mathrm{MPa})\end{array}$} & $\begin{array}{c}\text { Measured Creep Rate } \\
(\mathrm{M} / \mathrm{m} \cdot \mathrm{h})\end{array}$ \\
\hline & 110 & $6.23 \times 10^{-8}$ \\
\hline & 135 & $\quad 1.0 \times 10^{-7}$ \\
\hline & 171 & $1.0 \times 10^{-7}$ \\
\hline & 203 & $1.36 \times 10^{-7}$ \\
\hline & 241 & $2.43 \times 10^{-7}$ \\
\hline & 260 & $3.61 \times 10^{-7}$ \\
\hline [a] $\mathrm{c}$ & $\begin{array}{l}\text { ladding temperatur } \\
\text { lux was } 2.9 \times 10^{17}\end{array}$ & $\begin{array}{l}6 \mathrm{~K} \text { and fast neutron } \\
\text { in all cases. }\end{array}$ \\
\hline
\end{tabular}


(2) Stress Dependence. It is clear that a high stress will give a high creep rate, but because of the anisotropy of zircaloy and the complex internal stress interactions within a stressed tubular specimen, this independence is not necessarily linear, although several investigators have reported this to be the case $[26,28,29,30]$. Several authors, such as Wood $^{[31]}$, Busby and White ${ }^{[32]}$, Fidleris and Williams ${ }^{[33]}$, and Pankaskie ${ }^{[34]}$ use hyperbolic functions. A popular form is $\dot{\epsilon} \propto \sigma^{\mathrm{n}}$ with $\mathrm{n} \neq 0$. This is used by (a) Coleman ${ }^{[35]}$, where $90 \longleftarrow \mathrm{n} \longleftarrow 10$; (b) $\mathrm{Kohn}^{[36]}$, where $5.3 \longleftarrow \mathrm{n} \longleftarrow 2$; (c) Azzarto et al ${ }^{[37]}$, where $n \simeq 4$; (d) Fidleris ${ }^{[38]}$, where $n \approx 3$; (e) Wood and Watkins ${ }^{[39]}$, where $10 ₹ \mathrm{n} \approx 50$; and many others. Many of the data indicate that the strain rate is linear with applied stress for relatively low stress and then exhibit a stronger stress dependence as the stress increases. For example, Bernstein ${ }^{[40]}$ found that the stress dependence of zircaloy-2 was proportional to $\sigma^{n}$, where $\mathrm{n}=1$ at low stresses and increases: to 4.5 to 5.5 at higher stresses. Nichols ${ }^{[41]}$ provided more insight along these lines, hypothesizing the in-pile creep mechanisms and specifying their stress dependence. Some of the mechanisms identified by Nichols, in order of increasing stress, are:

(1) Preferential alignment of vacancy and interstital loops $(\dot{\epsilon} \propto \sigma)$

(2) Glide of dislocations $\left(\dot{\epsilon} \propto \sigma^{10}\right)$

(3) Radiation-enhanced glide of edge dislocations over radiation produced obstacles ( $\dot{\epsilon} \propto \sigma$ at low $\sigma$ to $\dot{\epsilon} \propto \sigma^{4}$ at high $\sigma$ )

(4) Destruction of radiation obstacles $\left(\dot{\epsilon} \propto \sigma^{100}\right)$.

This dramatic increase in the stress dependence suggests an exponential function, which of course is also described by the hyperbolic forms cited earlier. The data fit best to an expression of the form $\left(\sigma+A e^{-\beta \sigma}\right)$, where $\beta$ is small enough that the linear stress dependence at low stresses $(\sigma<200 \mathrm{MPa})$ is not disturbed, but the general increase in $\dot{\epsilon}$ with $\sigma$ at high stresses is properly reflected.

(3) Fast Flux Dependence. Although most investigators agree that a fast flux. environment enhances the zircaloy creep rate and that the enhancement is of the form $\phi^{a}$, where $\phi$ is the fast flux, there is disagreement about the magnitude of the exponent a. Fidleris $^{[42]}$, Duffin and Nichols ${ }^{[43]}$, Piercy ${ }^{[44]}$, Ross-Ross and Hunt ${ }^{[30]}$, and Ross-Ross and Fidleris ${ }^{[26]}$ suggest that $\dot{\epsilon}$ is directly proportional to the fast flux. Wood ${ }^{[31,39]}$ uses $\dot{\epsilon} \propto \phi^{0.85}, \mathrm{Kohn}{ }^{[36]}$ uses $\phi^{0.65}$, and Gilbert ${ }^{[45]}$ uses $\phi^{0.5}$. However, several authors have noted that irradiation-induced creep is overshadowed by thermaly activated creep at high temperatures. Among these are Nichols ${ }^{[29,41]}$, Fidleris and Williams ${ }^{[33]}$, and Kohn ${ }^{[36]}$. Fidleris and Williams give the transition temperature as $523 \mathrm{~K}$ and Nichols as about $623 \mathrm{~K}$. Some authors, such as Pankaskie, give two correlations, one for high temperature and one for low temperature, with the transition being between 600 and $625 \mathrm{~K}$. A logical approach seems to be that of $\mathrm{Kohn}^{[36]}$, who models the flux dependence in the form $\mathrm{K} \mathrm{T}^{-7} \phi^{0.65}$. This has the virtue of cutting off the flux term at high temperatures as well as accounting for the fact that the higher the flux level is, the higher the temperature must be to anneal 
out the irradiation damage. The expression used in the correlaton is $\left(1+10^{8} \mathrm{~T}^{-7} \phi^{0.65}\right)$. The exponent 0.65 was chosen because it gave the best fit to the data. The 1 is added to the temperature-dependent term to provide calculational continuity at zero flux and to allow proper modeling of thermal creep at high temperatures.

(4) Temperature Dependence. In addition to the temperature dependence in the flux term, there is a creep activation energy to be overcome by thermal energies. As is usual for such cases, this activation energy is modeled using an Arrhenius function. Because several creep mechanisms are different, authors have reported different values for this activation energy, ranging from over $10^{5} \mathrm{~J} / \mathrm{mol}^{[36,40,46]}$ to less than $10^{4} \mathrm{~J} / \mathrm{mol}{ }^{[28]}$. Often, authors reported different activation energies in different temperature ranges $[36,42]$ but, in the interest of calculational simplicity, only a single activation energy has been used here. The value giving the best fit was $4.2 \times 10^{4} \mathrm{~J} / \mathrm{mol}$.

(5) Creep Rate Correlation. The correlation used to model the creep rate is:

$$
\dot{\varepsilon}=\frac{5.7 \times 10^{-12}\left(1+10^{8} T^{-7} \phi^{0.65}\right)\left(\sigma+710 \mathrm{e}^{4.97 \times 10^{-8} \sigma}\right)\left(\mathrm{e}^{-42,000 / R T}\right)}{\sqrt{t}}
$$

$$
\begin{aligned}
\dot{\epsilon} & =\text { diametral creep strain rate }(\mathrm{m} / \mathrm{m} \cdot \mathrm{s}) \\
\mathrm{T} & =\text { temperature }(\mathrm{K}) \\
\phi & =\text { fast neutron flux }(>1 \mathrm{MeV})\left(\text { neutrons } / \mathrm{m}^{2} \cdot \mathrm{s}\right) \\
\sigma & =\text { stress }(\mathrm{Pa}) \\
\mathrm{R} & =\text { universal gas constant }(8.314 \mathrm{~J} / \mathrm{mol}-\mathrm{k}) \\
\mathrm{t} & =\text { time }(\mathrm{s}) .
\end{aligned}
$$

2.2.3 Data Comparison. The most important data set, since it was the only one which met all of the criteria, is that gleaned from the Saxton reports $[21,22,23]$. A comparison with these data is shown in Figure 75, where the 627 and $629 \mathrm{~K}$ curves are approximated by a single line calculated for $628 \mathrm{~K}$. The agreement between the calculated curves and the data is excellent for the three lowest temperatures, but at the two higher temperatures, the correlation substantially overpredicts the creep rate. The discrepancy for these two data is not considered serious, because many of the rods from which these data were taken showed pellet-cladding interaction. Since the creep rate was obtained by simply taking the total creep and dividing by the time in pile, the cladding may not have been creeping for an appreciable fraction of the time due to the pellet-cladding interaction, thus giving unrealistically low creep rates. More of the $633 \mathrm{~K}$ rods showed pellet-cladding interaction than the $629 \mathrm{~K}$ rods, which in turn showed more than twice that of the $627 \mathrm{~K}$ rods. The 614 and the $621 \mathrm{~K}$ rods showed no pellet-cladding interaction. The predictions of the correlation are entirely in agreement with these considerations. 


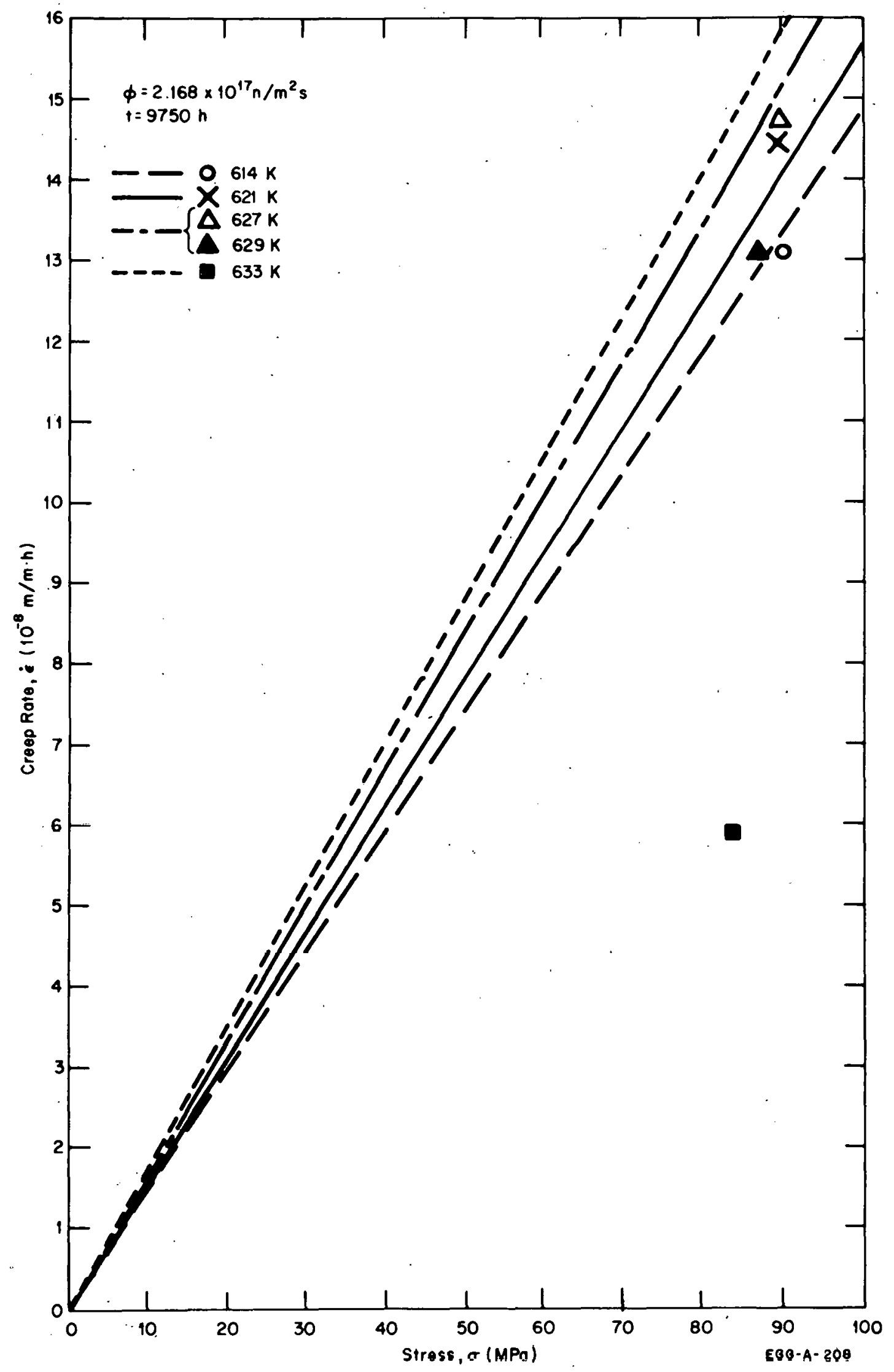

Fig. 75 Comparison of the predictions of the cladding creep rate equation with the Saxton ${ }^{[2,3,4]}$ data. 
Ibrahim's zircaloy-2 data ${ }^{[24]}$ are another data set which essentially. meets the criteria. It is of typical PWR dimensions, biaxially stressed, and was taken in pile. However, it is different from the Saxton data in that the rods were internally, rather than externally, pressurized, and the texture was typical of pressure tube material rather than of cladding material. The comparison of these data with the correlation is shown in Figure 76. The solid line is the prediction of Equation (4) and somewhat overpredicts the creep rate. This is consistent with the observation of Kohn [17] that pressure tubes do not creep as fast as cladding. The dashed line was calculated by multiplying Equation (4) by 0.8 and fits the data quite well. In addition, Figure 76 is useful in that it illustrates how the exponential part of the stress dependence causes a severe departure from a linear relationship between strain rate and stress beginning at a stress of about $200 \mathrm{MPa}$.

\subsection{Local Strains at Runture \\ D. L. Hagrman}

$\Lambda$ new model has bccn devcloped tó pıedicl lucal strains al failure of yircaalny ciladiding in the temperature range 1050 to $1370 \mathrm{~K}$. The model is intended for use with computer subroutines like BALOON ${ }^{[47]}$, which predict local strains as a function of temperature. The use of failure criteria based on local strain will provide improved predictions of tubing deformation at burst, because circumferential variations in temperature can be expected to cause variations in the distribution of strain around the cladding circumference.

2.3.1 Summary. The correlation for local strain at failure is preliminary since it is based on only seven cross sections of tubes that were burst at temperatures between 1090 and $1320 \mathrm{~K}$. However, surprisingly consistent results were obtained with these few tests. Local circumferential and local radial (wall thickness) strain at rupture are found to be closely approximated with linear functions of temperature. This implies that the, pronounced minimum in average circumferential expansion during the alpha-to-beta phase transition ${ }^{[48]}$ is a localization of circumferential strain rather than a reduction of the local strain required for rupture. Because local circumferential strain and local radial strain are related during deformation and at rupture, either component of strain is a candidate for use as a failure criterion. Both strains to rupture are developed here, but the change in radial strain is based on simple direct measurements and is therefore the preferred criterion for failure when cladding wall thickness can be determined. The local circumferential strain is discussed so it may be compared with time-dependent data which may be obtained from photographs of grids attached to cladding.

The true local radial (wall thickness) and true local circumferential strains ${ }^{\text {la }}$ at rupture are given by the correlations:

$$
\text { Circumferential strain }=-0.415+0.001368 \mathrm{~T}
$$

[a] True strain is the change in length divided by the length at the instant of change and integrated from initial to final length. 


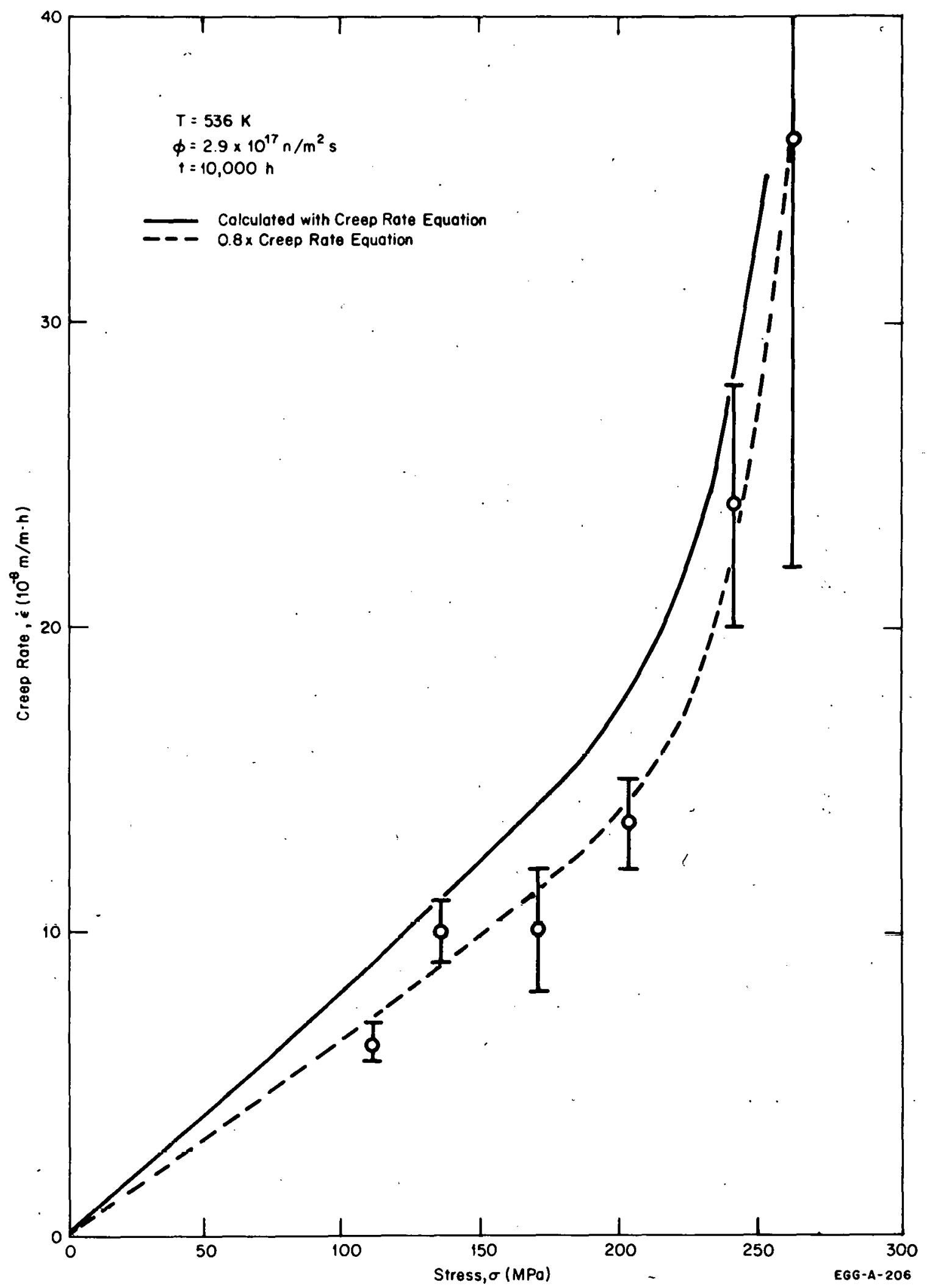

Fig. 76 Comparison of the İbrahim's $[3]$ pressure tube data with the prediction of the cladding creep rate equation. 
where $T$ is temperature in kelvins.

Corresponding values of engineering strain ${ }^{[a]}$ can be calculated with the expression

$$
\text { Engineering strain = exponent (true strain) }-1 \text {. }
$$

2.3.2 Derivation of Expressions Used for Local Strain. Photographs of cross sections taken perpendicular to the axis of several zircaloy tubes burst at Argonne National Laboratory $[49,50,51,52]$ were used to determine strain components at cladding rupture. Expansion of the tubes was assumed to consist of two parts. The first part, uniform expansion, is assumed to be symmetric in the plane of the tubing cross section ${ }^{[\mathrm{b}]}$ and the second part, ballooning, is assumed to consist of equal axial and circumferential strains localized on the side of the tube which burst. Neither of these assumptions has been verified, but neither is necessary to determine radial strains. The assumptions will affect only the relative values of circumferential and axial strains.

In order to find local uniform strains; the center of curvature of the side of the tube away from the burst was located by drawing chords on the side of the cross section opposite the burst and constructing perpendiculars to the chords as illustrated in Figure 77 . The uniform circumferential strain was calculated by comparing the circumference of the circle constructed at the center of curvature to the circumference of the original tubing ${ }^{[50]}$. Local strain was assumed to be equal to the average circumferential strain. Local radial strain during uniform deformation was computed from measurements of cladding wall thickness in the circular part of the cladding cross section and published values ${ }^{[51]}$ of the initial wall thickness. Local axial uniform strain was calculated using the incompressibility relation ${ }^{[c]}$

$$
\text { exponent }\left(\varepsilon_{\theta}\right) \text { exponent }\left(\varepsilon_{Z}\right) \text { exponent }\left(\varepsilon_{r}\right)=1
$$

where $\epsilon_{\theta}, \epsilon_{\mathrm{Z}}$, and $\epsilon_{\mathrm{r}}$ are the circumferential, axial, and radial components of local true strain.

[a] Engineering strain = change in length divided by the original length.

[b] The assumption of symmetric deformation during the first part of these tests may be reasonable, because circumferential variation in temperature should be minimized by the use of self-resistance heating in the tests described here.

[c] In terms of engineering strains, the relation is $\left(1+e_{\theta}\right)\left(1+e_{Z}\right)\left(1+e_{r}\right)=1$, where $e_{\theta}$, $e_{Z}$, and $e_{r}$ are the circumferential, axial, and radial components of local engineering strain. 


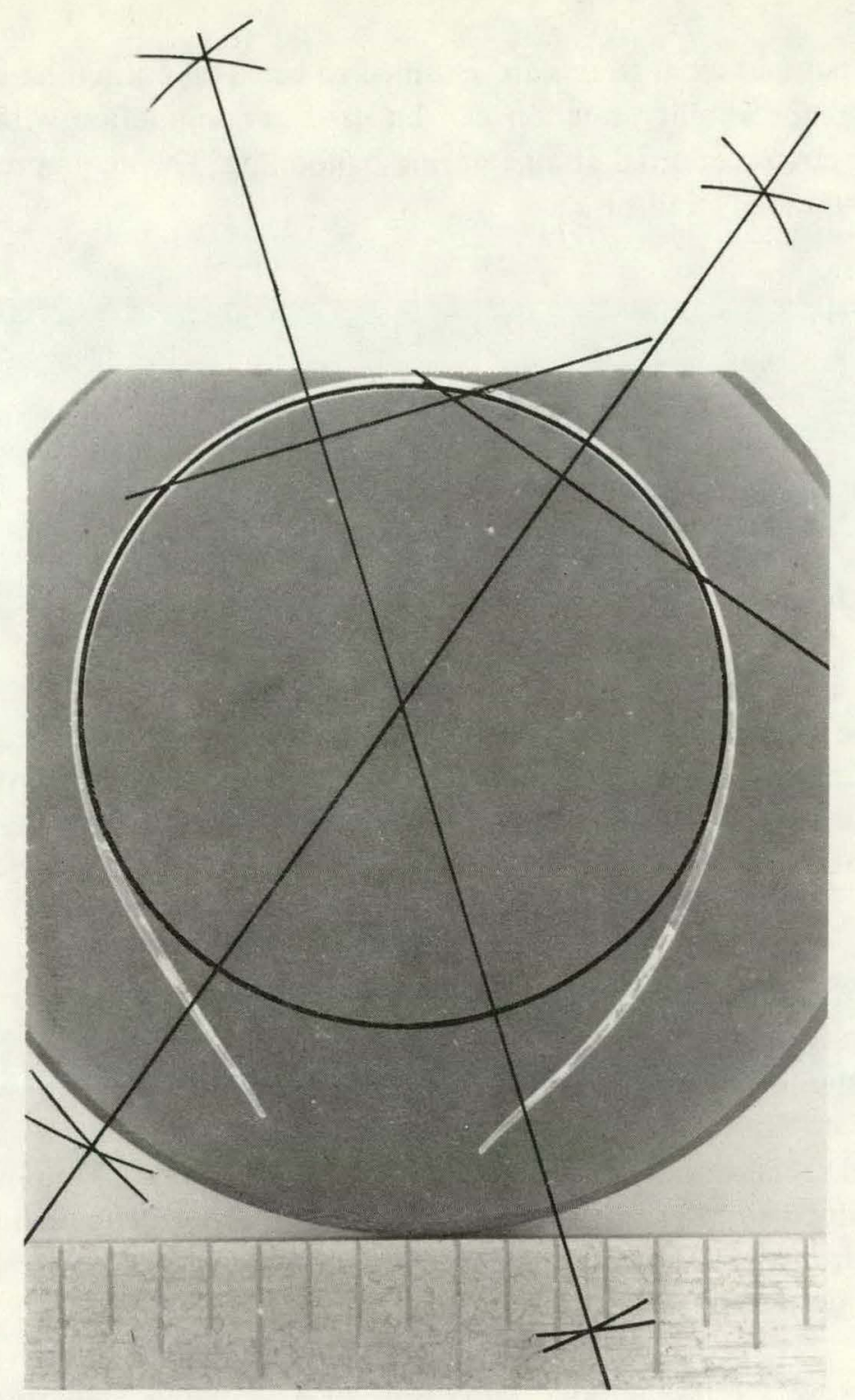

Fig. 77 Lucation of the center of curvature of the side of the tube away from the burst region.

Strains during the ballooning part of the deformation were calculated by measuring the wall thickness adjacent to the burst. Most burst edges displayed a fracture-like line approximately $45^{\circ}$ from the radial direction. The wall thickness was measured adjacent to this line or, if the line could not be distinguished, $0.01 \mathrm{in}$. from the burst tear. The radial strain during the ballooning part is then given by

where

$$
\varepsilon_{r}{ }^{\prime}=\text { natural } \log \frac{W_{\text {burst }}}{W_{\text {uniform }}}
$$

$$
\begin{aligned}
& \epsilon_{\mathrm{r}}^{\prime} \quad=\text { true local radial strain during the ballooning deformation } \\
& \mathrm{W}_{\text {burst }}=\text { wall thickness adjacent to the burst } \\
& \mathrm{W}_{\text {uniform }}=\text { wall thickness after uniform deformation. }
\end{aligned}
$$


Since circumferential and axial strains are assumed to be equal during the ballooning part of the burst, the incompressibility relation can be used in conjunction with Equation (9) to find the axial and circumferential strains during ballooning. The incompressibility equation with equal axial and radial strains is

$$
\varepsilon_{\theta}^{\prime}=\frac{1}{2} \varepsilon_{r}{ }^{\prime}
$$

where

$$
\begin{aligned}
& \epsilon_{\theta}{ }^{\prime}=\text { the true circumferential strain during ballooning } \\
& \epsilon_{\mathrm{r}}{ }^{\prime}=\text { the true radial strain during ballonning. }
\end{aligned}
$$

True strains are additive, so the net local components of strain at rupture are the sum of the uniform and ballooing parts of each component. These sums, the burst temperatures, and the heating rates for seven bursts are shown in Table XIX. Although there is considerable scatter in the uniform and ballooning parts of the strain, there is an apparent correlation of both the net circumferential and net radial strains at rupture with temperature.

Figure 78 illustrates the correlation of the components of true local strain at rupture with burst temperature. The local circumferential strains at rupture increase linearly with temperature, and the local radial strains decrease (become more negative) with temperature. The predictions of Equations (5) and (6), which are used to represent true local circumferential and radial strains, are also illustrated. These equations are linear least squares fits to the seven values of the circumferential and axial strains which are illustrated. The one test that was performed at a heating rate of $5 \mathrm{~K} / \mathrm{s}$ does not deviate from the trend of the other tests, which represent heating rates of approximately $115 \mathrm{~K} / \mathrm{s}$.

\subsection{Axial Gas Flow Experiments: Comparison of Calculated and Measured Gas Flow Gaps}

S. J. Dagbiartsson, B. A. Murdock, D. E. Owen

'Transient and steady state axial gas flow tests have been performed on irradiated commercial PWR fuel rods ${ }^{[6]}$. The steady state results were used to calculate effective radial fuel-to-cladding gap widths by employing the mass flow model used in two fuel performance codes, FRAP-T2 ${ }^{[53]}$ and SSYST ${ }^{[54]}$ :

$$
W=\frac{\pi}{H a} \bar{D} D_{H}{ }^{3} \frac{P_{1}^{2}-P_{2}^{2}}{2 \eta R T L}
$$


TABLE XIX

COMPONENTS OF LOCAL TRUE STRAIN

\begin{tabular}{|c|c|c|c|c|}
\hline \multirow{2}{*}{$\begin{array}{c}\text { Burst Temperature } \\
(\mathrm{K})\end{array}$} & \multirow{2}{*}{$\begin{array}{l}\text { Heating Rate } \\
(\mathrm{K} / \mathrm{S})\end{array}$} & \multicolumn{3}{|c|}{ True Strains } \\
\hline & & Type Strain & Circumferential & Radial Axial \\
\hline 1089 & 5 & $\begin{array}{l}\text { Uniform } \\
\text { Balloon } \\
\text { Total }\end{array}$ & $\begin{array}{l}0.71 \\
0.41 \\
1.12\end{array}$ & $\begin{array}{lr}-0.49 & -0.23 \\
\frac{-0.82}{-1.31} & \frac{0.41}{0.18}\end{array}$ \\
\hline 1129 & 115 & $\begin{array}{l}\text { Uniform } \\
\text { Balloon } \\
\text { Total }\end{array}$ & $\begin{array}{l}0.45 \\
0.55 \\
1.00\end{array}$ & $\begin{array}{lr}-0.19 & -0.26 \\
\frac{-1.11}{-1.30} & \frac{0.55}{0.29}\end{array}$ \\
\hline 1138 & 115 & $\begin{array}{l}\text { Uniform } \\
\text { Balloon } \\
\text { Total }\end{array}$ & $\begin{array}{l}0.67 \\
0.64 \\
1.31\end{array}$ & $\begin{array}{ll}-0.46 & -0.21 \\
\frac{-1.28}{-1.74} & \frac{0.64}{0.43}\end{array}$ \\
\hline 1164 & 115 & $\begin{array}{l}\text { Uniform } \\
\text { Balloon } \\
\text { Total }\end{array}$ & $\begin{array}{l}0.30 \\
0.77 \\
1.07\end{array}$ & $\begin{array}{rr}-0.09 & -0.21 \\
-1.54 & 0.77 \\
-1.63 & \frac{0.56}{0}\end{array}$ \\
\hline 1278 & 130 & $\begin{array}{l}\text { Uniform } \\
\text { Balloon } \\
\text { Total }\end{array}$ & $\begin{array}{l}0.67 \\
0.70 \\
1.37\end{array}$ & $\begin{array}{ll}-0.33 & -0.34 \\
\frac{-1.40}{-1.73} & \frac{0.70}{0.36}\end{array}$ \\
\hline 1348 & 115 & $\begin{array}{l}\text { Uniform } \\
\text { Balloon } \\
\text { Total }\end{array}$ & $\begin{array}{l}0.72 \\
0.60 \\
1.32\end{array}$ & $\begin{array}{ll}-0.43 & -0.29 \\
\frac{-1.20}{-1.63} & \frac{0.60}{0.31}\end{array}$ \\
\hline 1369 & 115 & $\begin{array}{l}\text { Uniform } \\
\text { Balloon } \\
\text { Total }\end{array}$ & $\begin{array}{l}0.85 \\
0.70 \\
1.55\end{array}$ & 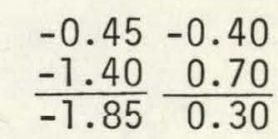 \\
\hline
\end{tabular}

where

$$
\begin{aligned}
& \mathrm{W}=\text { mass flow rate }\left(\mathrm{kg} / \mathrm{m}^{2} \cdot \mathrm{s}\right) \\
& \bar{D}=\text { the mean diameter between fuel surface and cladding ID }(\mathrm{m}) \\
& \mathrm{D}_{\mathrm{H}}=\text { hydraulic diameter }=\text { the diametral gap size }(\mathrm{m}) \\
& \eta=\text { gas viscosity }(\mathrm{Pa}-\mathrm{s}) \\
& \mathrm{R}=\text { universal gas constant }
\end{aligned}
$$




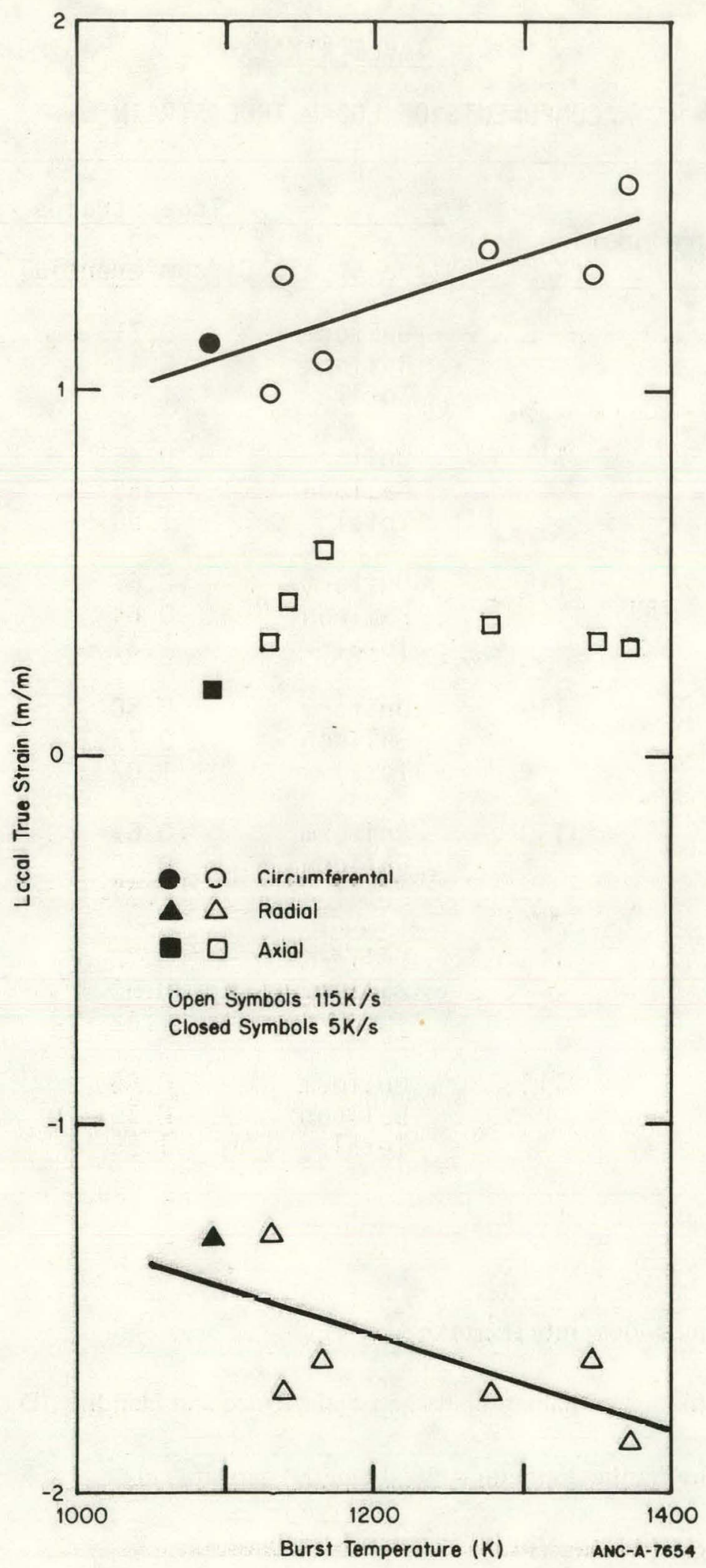

Fig. 78 Components of true local strain at rupture. 


$$
\begin{aligned}
& \mathrm{T}=\text { absolute temperature }(\mathrm{K}) \\
& \mathrm{L}=\text { flow path length }(\mathrm{m}) \\
& \mathrm{Ha}=\text { Hagen number }=64 \text { for tubular gap, } 96 \text { for plane gap. }
\end{aligned}
$$

The effective radial gap $(\Delta r)$ is calculated by using the following definitions:

$$
\begin{aligned}
& \Delta \mathrm{r}=1 / 2 \mathrm{D}_{\mathrm{H}} \\
& \overline{\mathrm{r}}=1 / 2 \overline{\mathrm{D}} \\
& \mathrm{Ha}=96
\end{aligned}
$$

and rearranging Equation (11) to yield

$$
\Delta r^{3}=\frac{W 12 \eta R T L}{\bar{r} \pi\left(P_{1}^{2}-P_{2}^{2}\right)} .
$$

Equations (11) and (12) assume an ideal, laminar compressible gas flow through a constant rod gap.

The steady state test results were used in Equation (12) to calculate the local effective gap as a function of rod position. These results were previously reported ${ }^{[6]}$.

Several metallographic sections were taken near the top of Rod K-4 to compare the calculated effective gaps with gaps measured from metallographic photographs. Uranium dioxide fuel fractures and relocates during operation and the resultant cracks may contribute to the internal gas flow paths. However, the gas flow model from which Equations (11) and (12) are derived assumes annular gap gas flow only. Therefore, the observed fuel gas paths, both annular gaps and fuel cracks, must be reduced to an average effective annular gap for model comparison.

The data obtained from the metallographic photographs (examples of which are shown in Figure 79) were used in two ways. First, the annular fuel-to-cladding gap was measured every $30^{\circ}$ around the sample circumference and averaged. The averaging procedure was based on the assumption that the total gas flow penetrates through many parallel annular gaps of different breadth $b_{i}=2 \pi r$, width $d_{i}=\Delta \bar{r}$, and possibly length $\Delta \mathrm{Z}_{\mathrm{i}}=\mathrm{L}$. Thus, the equation for the total mass flux reduces to

$$
w_{\text {tot }}=\frac{4\left(p_{1}^{2}-p_{2}^{2}\right)}{n R T} \quad \sum_{i=1}^{I} \frac{b_{i} d_{i}^{3}}{H a_{i} \Delta L_{i}}
$$




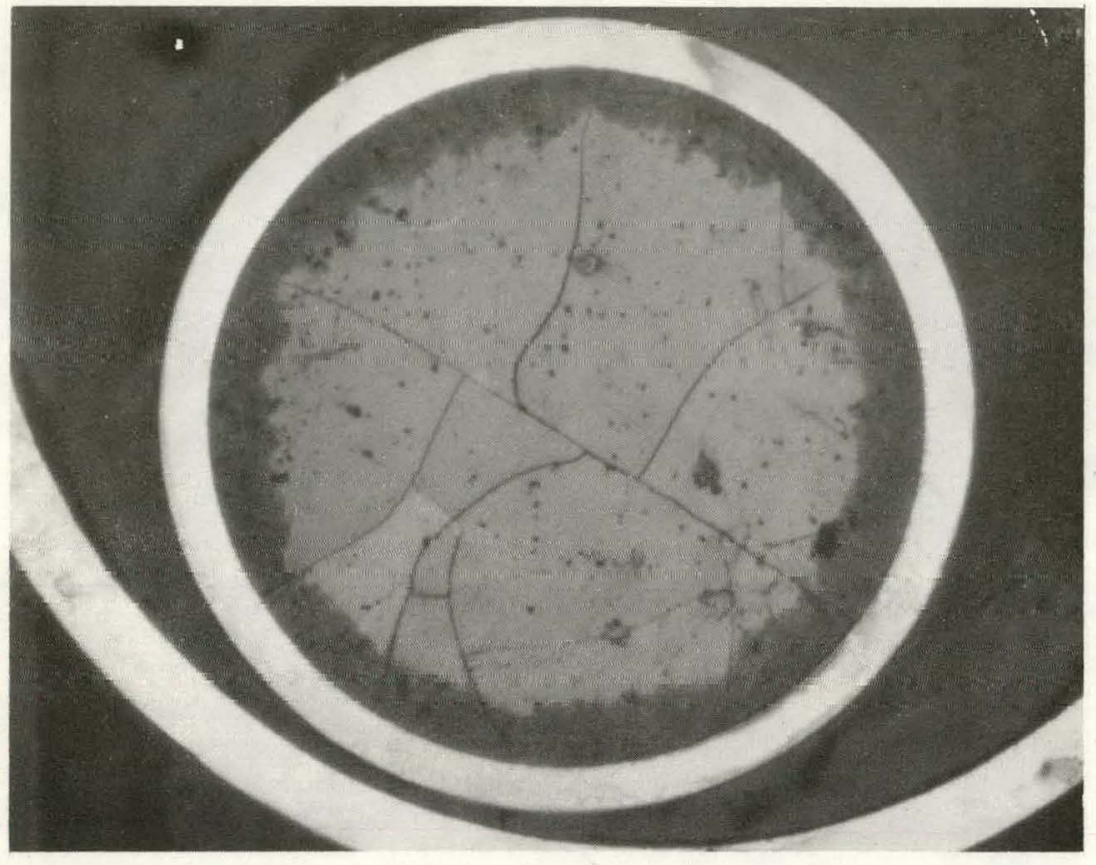

Rod K-4 Section D-5 (Macro)

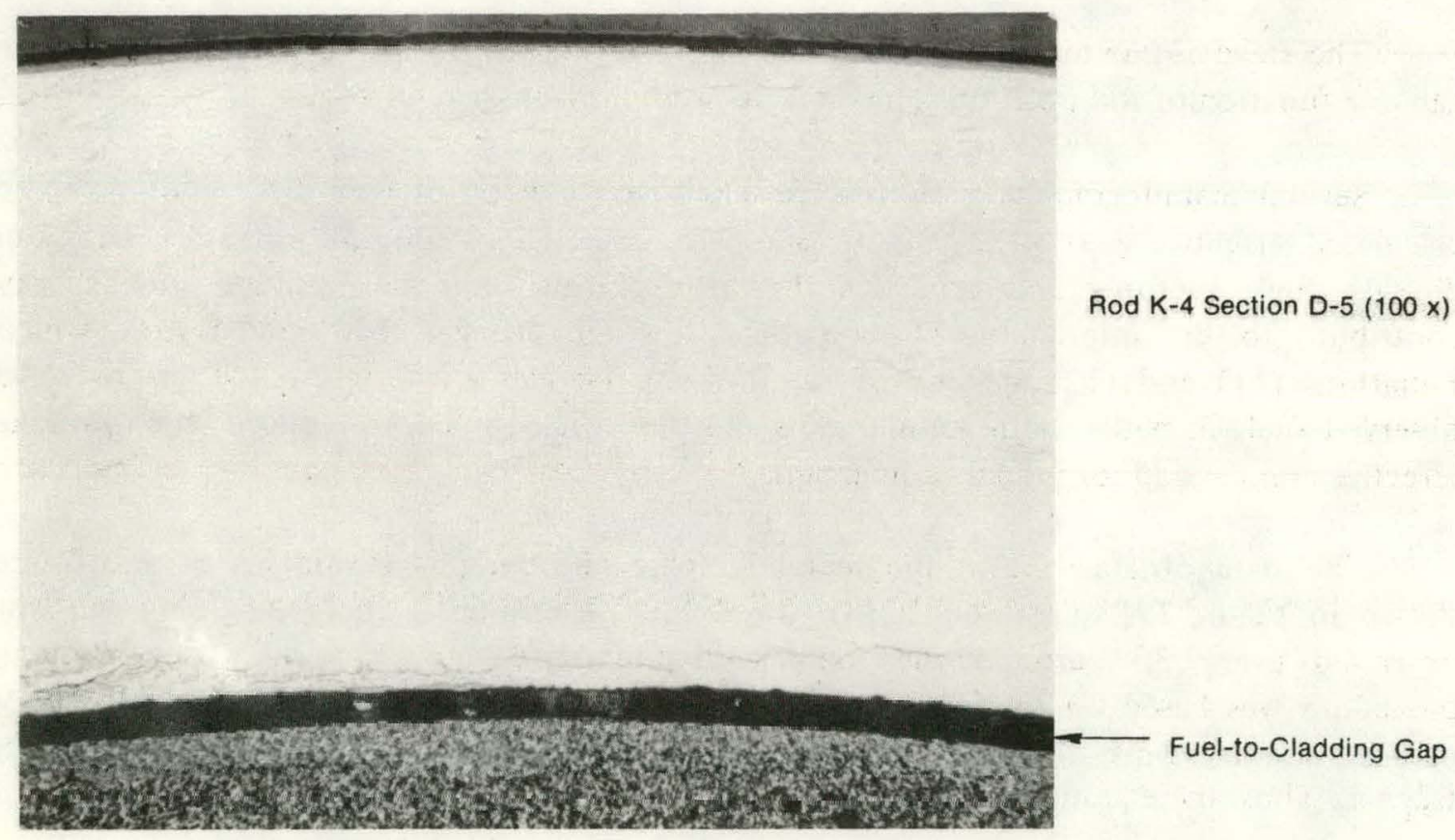

Fig. 79 Typical fuel crack pattern and fuel-to-cladding gap. 
The right hand sides of Equations (11) and (13) being equal resulted in the following equation for the average effective gap:

$$
\frac{\overline{\Delta r}^{3}}{\mathrm{Ha}}=\frac{\Delta \mathrm{Z}}{2 \pi \bar{r}} \quad \sum_{i=1}^{\mathrm{I}} \frac{\mathrm{b}_{i} d_{i}^{3}}{\mathrm{Ha}_{i} \Delta \mathrm{Z}_{i}}:
$$

Equation (14) is simplified by (a) considering the flow path length to be constant in any one fuel section (i.e., $\Delta Z_{i}=\Delta Z$ ); (b) assuming constant annular gap over a $30^{\circ}$ arc, thus the gap breadth reduces to $b_{i}=\pi \bar{r} / 6$; and (c) assuming that Hagen number for individual paths is constant (i.e., $\mathrm{Ha}_{\mathrm{i}}=\mathrm{Ha}$ ). Equation (14) then reduces to

$$
\overline{\Delta r}=\sqrt[3]{\sum_{i=1}^{12} \frac{\left(\Delta r_{i}\right)^{3}}{12}} .
$$

Furthermore, the contribution of the gas flow resulting from the fuel cracks can be determined. A quantitive determination is not possible, but the contribuiton of the major cracks can be estimated by measuring them and using an averaging procedure as above.

The results of the gas flow analysis and actual measurements are summarized in Table $X X$. The calculated effective annular gap was obtained by modifying the previously reported experiment results ${ }^{[6]}$ by a factor which accounts for the variation in Hagen number with gap size. The effect was to increase the earlier results by 20 to $40 \%$. Generally, the effective gaps calculated from the gas flow experiments are bracketed by the two measured effective gaps (annular gap only and annular gap plus cracks). There is a clear indication that the fuel cracks contribute substantially to total gas flow. The importance of fuel cracks to gas flow is even greater when it is recalled that the radial gap will substantially close in an operating fuel rod because of cladding creep-down, fuel expansion, and fuel relocation. 
TABLE. XX

COMFAR:SON OF CALCULATECI AND MEASURED. EFFECTIVE ANNULAP. GAPS IN ROD K-4

\begin{tabular}{|c|c|c|c|c|c|c|c|}
\hline \multirow[b]{2}{*}{ Measurement or Calculation } & \multicolumn{7}{|c|}{ Section Number } \\
\hline & $E-5$ & $\mathrm{E}-2$ & $E-1$ & $\mathrm{D}-5$ & D-3 & $\mathrm{D}-2$ & $\mathrm{D}-1$ \\
\hline Distance from top of rod (neasured, $m$ ) & $0.15:$ & 0.40 & 0.45 & 1.05 & 1.19 & 1.30 & 1.40 \\
\hline 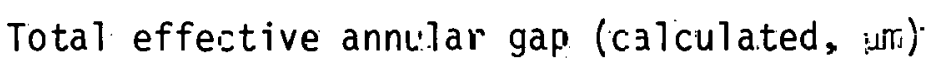 & $36-41$ & $36-41$ & $36-41$ & $29-31$ & $29-31$ & $29-31$ & $29-31$ \\
\hline $\begin{array}{l}\text { Effective annular gap imeacured, }{ }^{\mathrm{m}} \text { ) } \\
\text { (contribution of racia } \text { gaps only) }\end{array}$ & 31. & 34 & 34 & 21 & 28 & 15 & 31 \\
\hline $\begin{array}{l}\text { Effective annular gap (measured, um). } \\
\text { (contribution of fuel cracks anly) }\end{array}$ & 70 & 25 & 42 & 33 & 17 & 28 & 87 \\
\hline $\begin{array}{l}\text { Total effective annular gaf, (measured, } \mathrm{m} \text { ) } \\
\text { (sum of radial gap and fuel cracks) }\end{array}$ & $72^{[\mathrm{a}]}$ & 38 & 48 & 35 & 30 & 30 & 88 \\
\hline [a] $72^{3}=31^{3}+70^{3}$. & & & & & & & \\
\hline
\end{tabular}




\section{FUEL MODEL DEVELOPMENT AND VERIFICATION}

\section{J. A. Dearien}

Development of the Fuel Rod Analysis Program - Steady State (FRAP-S) and Fuel Rod Analysis Program - Transient (FRAP-T) codes continued. FRAP-T3 was verified, and the verification of FRAP-S3 was initiated. FRAP-S2 was transmitted to the Argonne Code Center for distribution to the nuclear industry.

Improvements were made to many of the subcodes of FRAP, with the most significant being to the mechanical analysis subcode FRACAS (Fuel Rod and Cladding Analysis Subcode) and the failure subcode FRAIL (FRAp Integrity Limits). A deformable pellet routine was added to FRACAS to calculate fuel creep and hot pressing at high temperatures. Subroutines were added to FRAIL to calculate transient flow blockage during a LOCA.

Details of the existing FRAIL subcode are covered in the following section.

\subsection{Status of the Fuel Rod Failure Subcode, FRAIL}

J. D. Kerrigan

A subcode has been developed for the FRAP codes[55] to predict failure of the zircaloy cladding of light water reactor fuels. This subcode, FRAIL, is a modular routine that predicts the probability of failure of fuel cladding under a variety of steady state and transient conditions.

The FRAIL subcode is composed of several subroutines, each of which has the capability of predicting zircaloy fuel rod cladding failure. The subroutines are distinguished from one another by mode of failure. A probability of failure is calculated for each mode, and these probabilities are then appropriately combined to yield a general probability of failure of the fuel rod cladding. The cladding failure models that are currently operable in FRAIL include:
(1) Cladding melt
(2) Oxide laycr wall thinning
(3) Ballooning (without rupture)
(4) Eutectic melt
(5) Collapse
(6) Overstress failure 
(7) Cumulative stress damage

(8) Overstrain failure.

This report is one in a continuing series of status reports on the development of the FRAIL subcode. Section 3.1.1 discusses a statistical comparison made between the empirical and assumed distributions of failure stress. A comparison of the Halden pellet-cladding interaction failure frequency and the overstress failure frequency as calculated by FRAIL is presented in Section 3.1.2. A new model in FRAIL, which is discussed in Section 3.1.4, is composed of a set of subroutines which calculate fractional flow blockage during core depressurization accidents.

3.1.1 Comparison Between Empirical and Assumed Distributionis of Failure Stress. One of the models currently being used in FRAIL is an empirical model that predicts the overstress failure of zircaloy-clad fuel rods. An empirical correlation between failure stress and temperature was derived using a least-squares fit of available data, given by:

$$
\log \sigma_{f}=5.00+3.27 \times 10^{-4}-1.15 \times 10^{-6} t^{2}+2.56 \times 10^{-10} t^{3}
$$

where

$$
\begin{aligned}
\sigma_{\mathrm{f}} & =\text { failure stress }(\mathrm{psi}) \\
\mathrm{t} & =\text { temperature }\left({ }^{\circ} \mathrm{F}\right)
\end{aligned}
$$

This correlation is plotted, along with the data, in Figure 80.

For the purpose of the FRAIL subcode, the probability of failure as a function of stress and temperature is needed. This is obtained by assuming a distribution of failure stress about the mean failure stress [as given by Equation (16)] .

From physical considerations; a beta distribution was chosen to represent the data. The beta distribution is limited to a finite interval and, hence, may be adjusted to include all available data. For a given temperature, then, values of stress which lie above the defined interval result in failure (probability of 1 ), while values which fall below the interval result in no failure (probability of 0 ). Because the beta distribution is defined only on the interval $(0,1)$, it is necessary to normalize both the failure stress and the standard deviation of the failure stress to this interval. By a simple variable transformation, the normalized failure stress is found from the expression:

where

$$
\bar{x}=\frac{\sigma_{F}-B}{T-B}
$$

$$
\begin{aligned}
& \overline{\mathrm{x}}=\text { normalized failure stress } \\
& \sigma_{\dot{\mathrm{F}}}=\text { mean failure stress }
\end{aligned}
$$




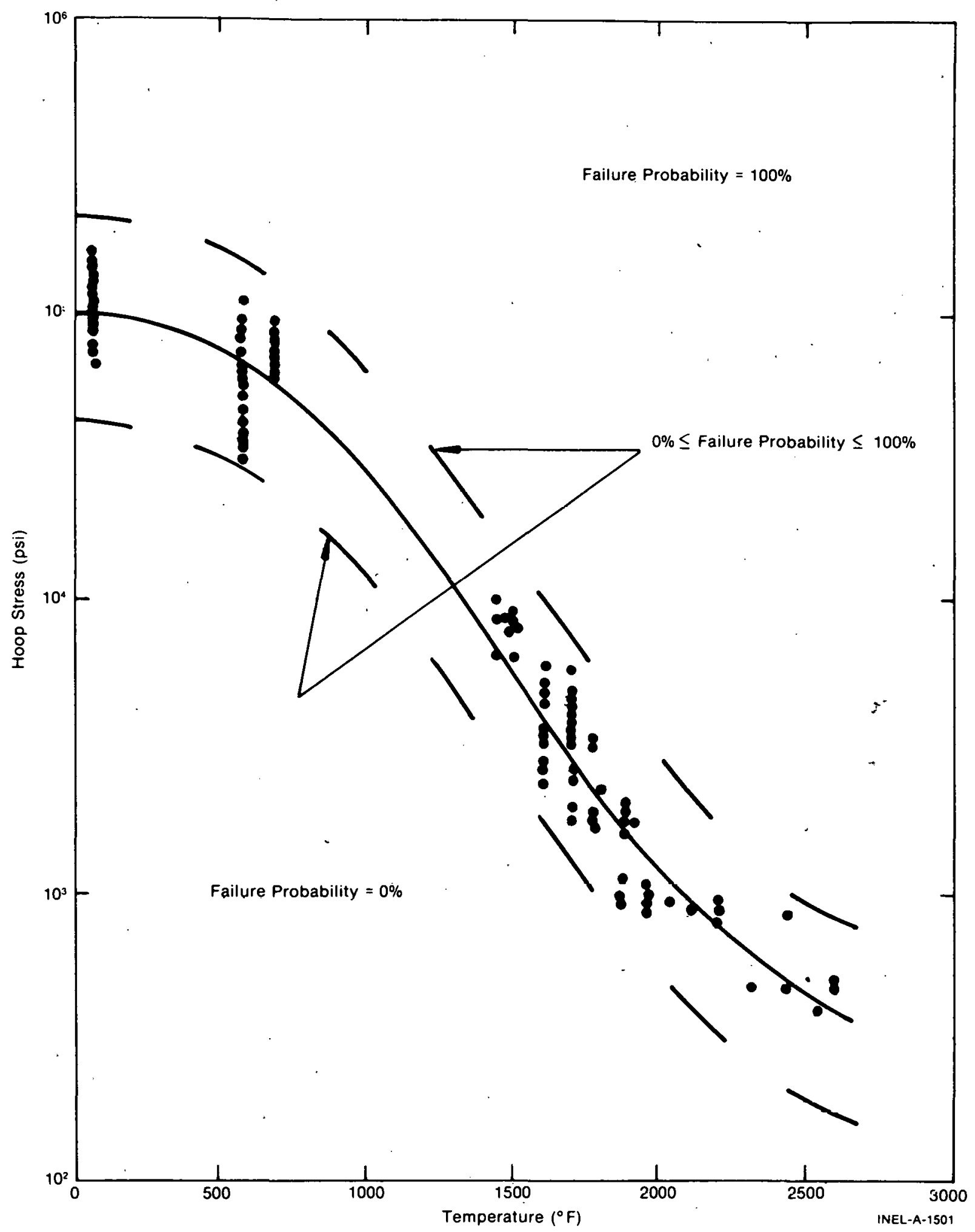

Fig. 80 Correlation between failure stress and temperature. 
and $\mathrm{B}$ and $\mathrm{T}$ define the interval of allowable failure stresses. This interval was chosen to be three standard deviations above and two standard deviations below the mean failure stress, so that for a given temperature, all available test data are included in the interval. These limits are depicted in Figure 80.

The normalized standard deviation is found from the equation [56]

where

$$
s=s^{\prime}\left(\frac{\partial \bar{x}}{\partial \sigma_{F}}\right)=\frac{s^{\prime}}{T-B}
$$

$\mathrm{s}=$ normalized standard deviation, that is, standard deviation of $\bar{x}$

$s^{\prime}=$ standard deviation of $\sigma_{\mathrm{F}}$.

Knowledge of $x$ and $s$ allows estimation of the two shape parameters $(\hat{\eta}$ and $\hat{\gamma})$ of the beta distribution. These estimates are found from the equations [57]:

$$
\hat{n}=\frac{(1-\bar{x})}{s^{2}}\left[\bar{x}(1-\bar{x})-s^{2}\right]
$$

and

$$
\hat{\gamma}=\frac{\bar{x} \hat{n}}{(1-\bar{x})} .
$$

From equations (19) and (20), $\hat{\eta}$ and $\hat{\gamma}$ were found to be 3 and 2 , respectively, for the overstress model.

By using the normalized failure strcss [defined in Equation (17)], it is possible to group all available data into one stress interval. In this way, the number of data points is large enough to allow a check on distributional assumptions.

An empirical cumulative distribution was constructed from the available data and is plotted in Figure 81. Also shown in Figure 81 is a cumulative beta distribution with $\eta=3$ and $\gamma=2$. As can be seen, the beta distribution closely approximates the emplrical distribution.

A chi-squared goodness-of-fit test was used to statistically evaluate the assumption that the failure stress data may be represented by a beta distribution with parameters $\eta=3$ and $\gamma=2$. The value of the computed test statistic was $\chi_{30}^{2}=42.3$. Since the computed test statistic does not exceed the 0.95 tabulated value of $\chi^{2}$ with 30 degrees of freedom $\left(\chi^{2}=43.8\right)$, this means that at the $95 \%$ confidence level there is no evidence of any difference between the empirical distribution and the assumed distribution.

As more data become available, it will be necessary to reevaluate the distributional assumptions. However, because of the large number of data used in this chi-squared test (222 observations), it would require a significant number of deviate observations to alter the validity of the assumed distribution. 


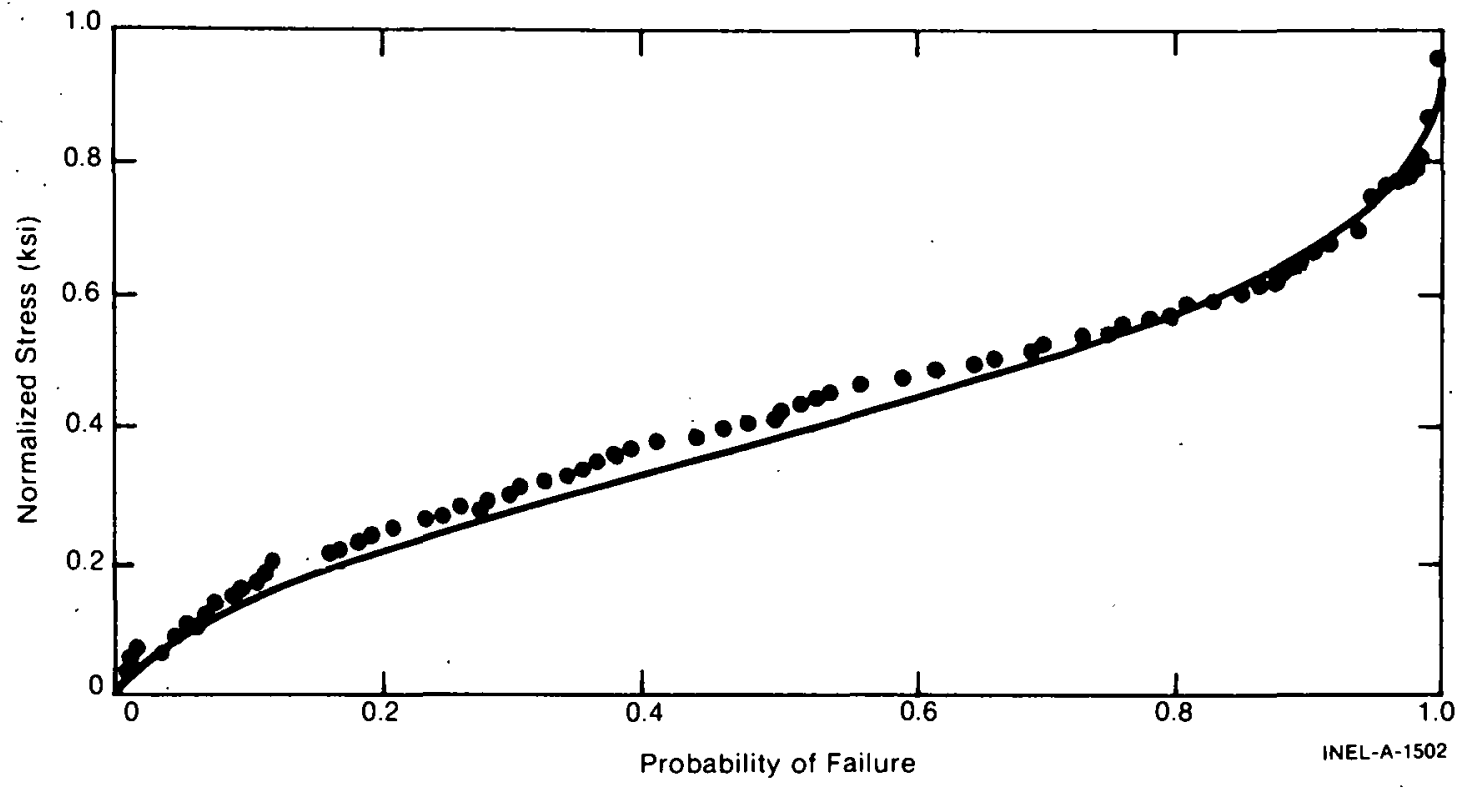

Fig. 81 Comparison of empirical and assumed distribution of failure stress.

3.1.2 Prediction of Pellet-Cladding Interaction Failures. During the FRAP-T2 model verification effort, it was determined that a strong correlation existed between high hoop stress as calculated by FRAP-T2, Version 5, and the failure frequency of the Halden rods [58]. FRAIL contains an overstress failure model, and a comparison of the FRAIL-3 prediction with this observed failure frequency was made.

Figure 82 shows a comparison between the FRAIL prediction of failure and the calculated failure probability of 44 Halden rods put through various power ramps. Because many of the Halden rods were not power ramped to failure, it is not possible to obtain the actual failure probability as a function of stress ratio. Only upper and lower limits on the failure probability may be found.

The bottom dashed line in Figure 82 is found by plotting the following function:

$$
P_{f}(s)=\frac{1}{N} \sum_{s} N_{f}(s)
$$

where

$$
\begin{aligned}
& \mathrm{P}_{\mathrm{f}}=\text { probability of failure } \\
& \mathrm{s}=\text { stress ratio } \\
& \mathrm{N}=\text { total number of rods }(=44) \\
& \mathrm{N}_{\mathrm{f}}=\text { number of observed failures at a given stress ratio. }
\end{aligned}
$$

Because all of the rods that did not fail certainly would have failed if they had been brought to a high enough stress ratio, Equation (21) defines the lower bound on the empirical failure probability. 


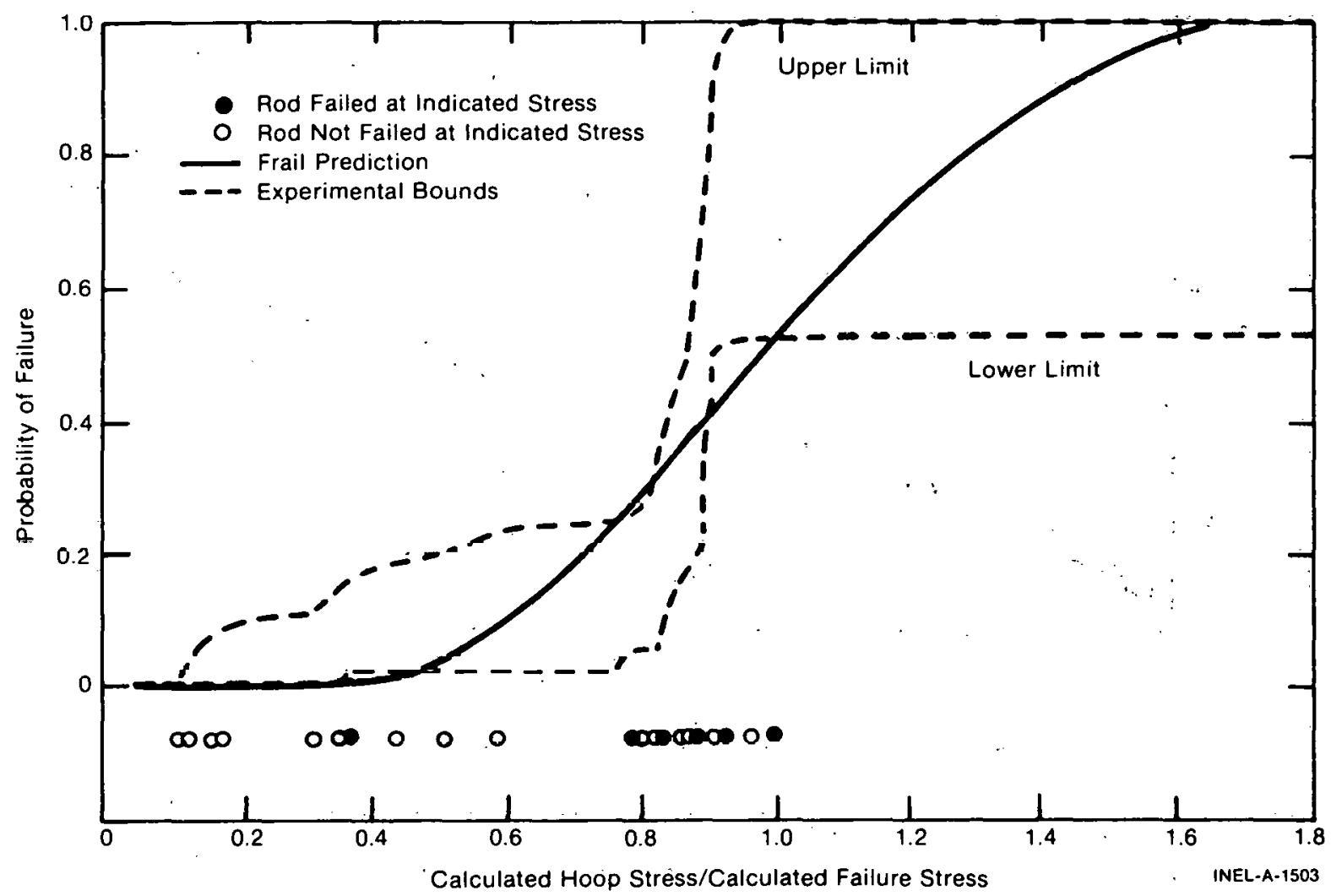

Fig. 82 FRAIL prediction of pellet-cladding interaction failures.

The upper dashed line in Figure 82 is found by assuming the "worst case," i.e., that all of the unfailed rods would fail in the next increment of stress. This defines the function:

where

$$
P_{f}(s)=\frac{1}{N} \sum_{s}\left[N_{f}(s)+N_{U}(s-\Delta s)\right]
$$

$\mathrm{N}_{\mathrm{u}}=$ number of unfailed rods which existed at the previous increment of stress.

As can be seen from Equation (22), this function defines an upper limit on the probability of failure as a function of stress ratio.

There are several important aspects of this comparison of the FRAIL prediction with the calculated failure probability of the Halden rods:

(1) The data base of the FRAIL model is from out-of-pile burst data and the experiment data are in-pile power ramp failures; therefore, this comparison is not merely a comparison of a model with its data base.

(2) The calculated hoop stress came from FRAP-T2, the calculated failure stress came from MATPRO, Version 006[59], and the initial conditions for FRAP-T2 were calculated with FRAP-S1, 
Version 002. Therefore, several different codes and models were exercised in obtaining the results, and the agreement in the final results seems to lend credence to the consistency of all of these models.

(3) The verification analysis of the overpower data suggests that rod failures are more associated with small gaps and high fuel density than with burnup. This supports the contention that stress dominates the failure mechanism in the low-temperature range. The correlation exists, then, between the operant experiment condition and the FRAIL model, which in this case considered only stress. This implies that the primary cause of failure was the same, namely, the high stresses present in both sets of data. This does not, however, imply that the mode of failure was the same.

3.1.3 Flow Blockage Calculations. The capability of predicting core flow blockage has been added to the FRAIL subcode. It is assumed that this routine would only be used during a high temperature transient in conjunction with a thermal-hydraulics code. Because of this, a "switch" has been added to FRAIL so that if flow blockage predictions are desired, no other failure mode is allowed.

(1) Percentage of Failed Rods. Before the flow blockage can be calculated, it is necessary to know the percentage of failed rods in the system. A correlation between failure stress and temperature was constructed from a large number of transient temperature burst data $[60]$. Although the burst tests were conducted in a number of different environments and at many different heating rates, there was no noticeable effect of these variables because of the large scatter in the data. The adopted correlation was of the form proposed by Chapman $[61]$ :

$$
T=\frac{5.02 E 4 \sigma}{3.71+0.71 \sigma}-0.03 \sigma+6.92 E 4
$$

where

$$
\begin{array}{ll}
\mathrm{T} & =\text { rupture temperature }\left({ }^{\circ} \mathrm{F}\right) \\
\sigma & =\text { stress }(\mathrm{psi}) .
\end{array}
$$

Equation (23) is valid in the temperature range of 1000 to $2900^{\circ} \mathrm{F}$ and is depicted in Figure 83.

In the high temperature rupture model, all tests were transient temperature burst tests. Initial pressure was measured and changes only slightly during the tests. In the high pressure region, this change is a very small percentage change, which implies that the pressure is fairly well known. Therefore, in this region, the uncertainty is assumed to be in the temperature measurement. At low pressures, the pressure change is a much larger percentage of the total pressure. Here, then, all uncertainty is assumed to be in the pressure measurement. 


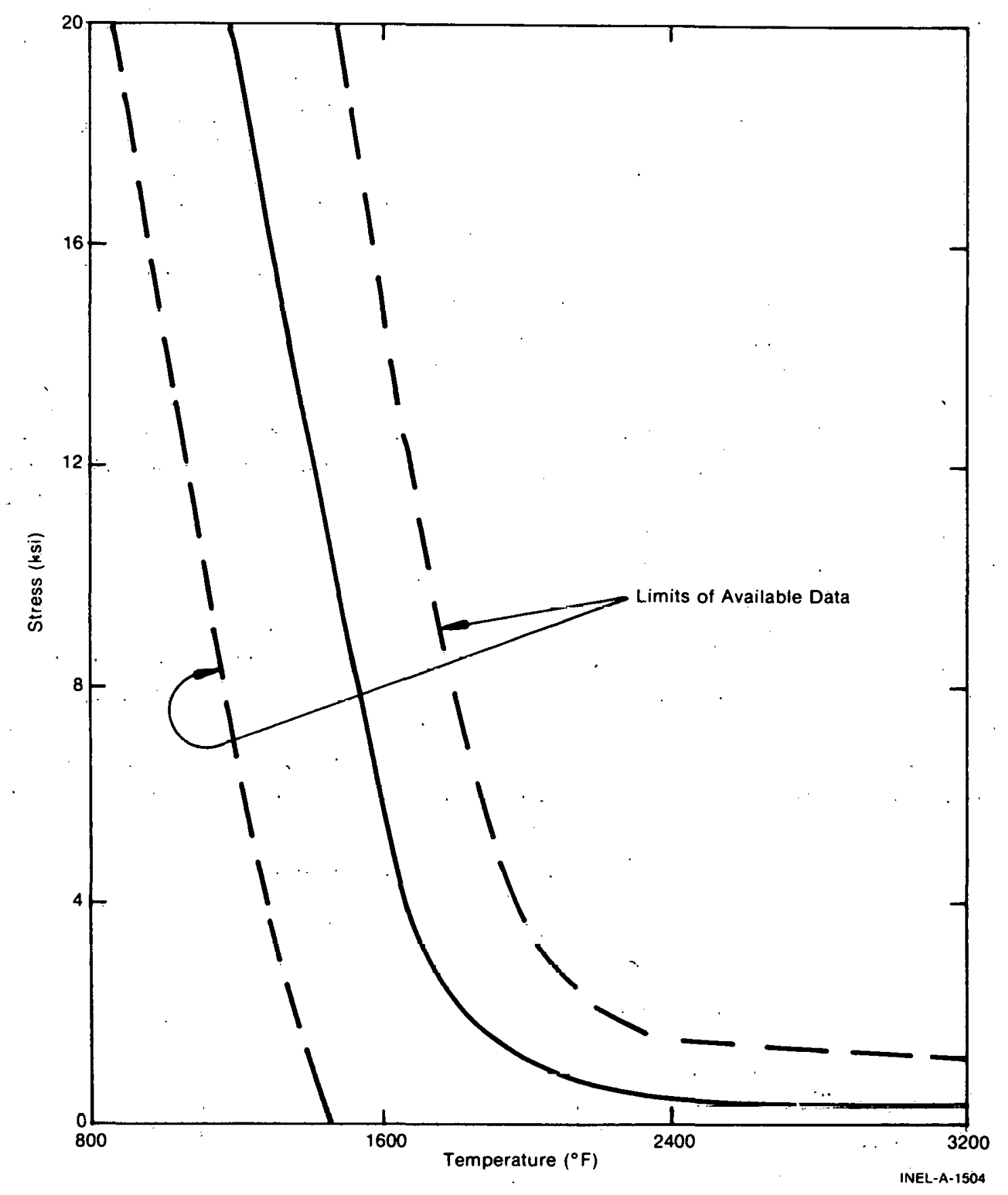

Fig. 83 Correlation between stress and temperature at failure. 
For the FRAIL subcode, a distribution of failure about the mean line must be defined. Above 2000 psi (where all uncertainty is assumed to be in temperature), the rupture temperature is assumed to be distributed according to the beta distribution; below $2000 \mathrm{psi}$, the failure stress is assumed to have the same distribution. The upper and lower limits of these distributions are chosen to include all available data and are shown as the dashed lines in Figure 83.

By defining the density distribuiton on the burst data, a probability of failure (or percentage of failed rods) may then be calculated for any stress-temperature point. The stress and temperature of the cladding is calculated by FRAP-T and transmitted to FRAIL. FRAIL then calculates the percentage of failed rods for use by the flow blockage subroutine.

(2) Flow Blockage. A correlation was developed between flow blockage fraction and cladding stress from Oak Ridge National Laboratory and Transient Reactor Test Facility LOCA data[62]. The data were found to be represented adequately by the equation of the form:

$$
\bar{F}_{A}=1.4-7.0 \times 10^{-4} \sigma+1.2 \times 10^{-7} \sigma^{2}-6.3 \times 10^{-12} \sigma^{3}
$$

where

$$
\begin{aligned}
& \overline{\mathrm{F}}_{\mathrm{A}}=\text { average fractional flow blockage in most highly blocked cross section } \\
& \sigma \quad=\quad \text { applied stress (psi). }
\end{aligned}
$$

The correlation represented by Equation (24) and the upper and lower distributional bounds are shown in Figure 84. Again, the distribution chosen to represent the data is a beta distribution. No check on the distributional assumptions could be made in this case because of the limited amount of data. As data from Chapman [61] become available, however, the statistical assumptions are easily revised, if necessary.

The following pertinent calculations may be made with the flow blockage routines:

(1) The probability of exceeding any specified flow blockage

(2) The flow blockage at any specified confidence level

(3) The average flow blockage

(4) The maximum possible flow blockage

(5) The minimum possible flow blockage.

The above listed quantities are all calculated as functions of time. The resulting flow blockages are valid only when all the fuel rods in a group being analyzed are similiar (i.e., all are undergoing the same temperature-pressure transient). 


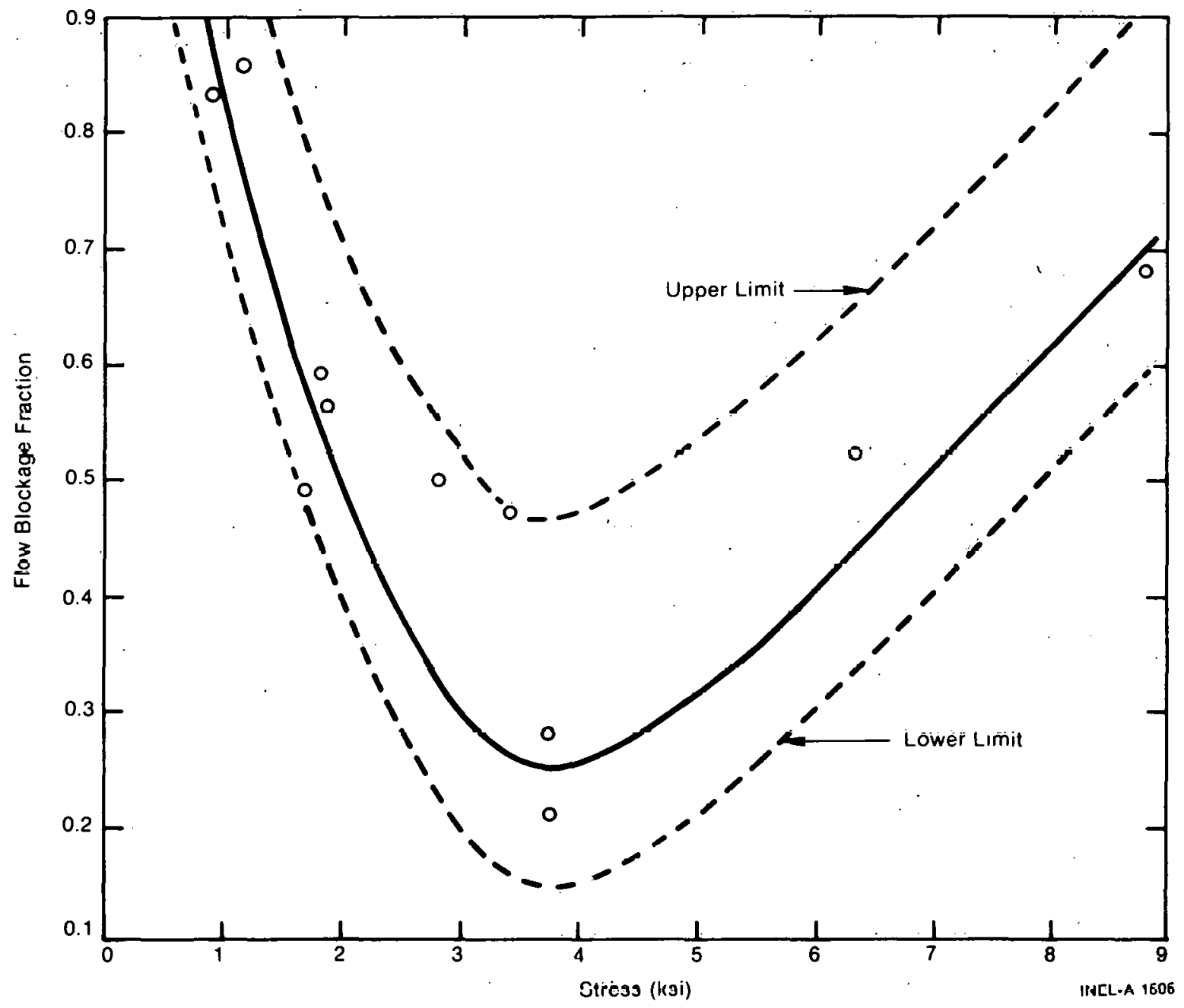

Fig. 84 Fiōw blóckage corièlation.

The following equation is used to calculate the average (best-estimate) flow blockage as a function of time:

$$
F \cdot(t)=F(t-\Delta t)+\bar{F}_{A}(t) p_{f}(t)
$$

where

$$
\begin{array}{ll}
\cdot F(t) \quad & \text { average flow blockage at time } t \\
F(t-\Delta t)= & \text { previous time step value of the average flow blockage } \\
\dot{F}_{A}(t)= & \text { average flow blockage from Equation (24) } \\
p_{f}(t)= & \text { percentage of rods which fail in the time step } t-\Delta t \text { to } t .
\end{array}
$$

By using Equation (25), FRAIL has the capability of calculating the flow blockage during a transient in which some rods survive. The assumption implicit in this equation is 
- that flow blockage accumulates in the same manner as the percentage of failed rods accumulates. Until transient flow blockage experiments are conducted, Equation (25) is assumed to be a valid representation of flow blockage as a function of time.

Another advantage to expressing flow blockage as in Equation (25) is that a constant pressure history need not be assumed. As the pressure changes during a problem, a fuel rod which fails during a particular time step will contribute a different amount to the total fractional flow blockage $\left(\overline{\mathrm{F}}_{\mathrm{A}}\right)$. This is because $\overline{\mathrm{F}}_{\mathrm{A}}$ is itself a function of pressure and, hence, of time.

Upper and lower bounds on the flow blockage are given by the following expressions:

$$
\begin{aligned}
& F_{u}(t)=F_{u}(t-\Delta t)+\bar{F}_{u}(t) p_{f}(t) \\
& F_{\ell}(t)=F_{\ell}(t-\Delta t)+\bar{F}_{\ell}(t) p_{f}(t)
\end{aligned}
$$

where

$$
\begin{aligned}
& \mathrm{F}_{\ell^{i}(\mathrm{t})}=\text { lower limit on flow blockage at time } \mathrm{t} \\
& \mathrm{F}_{\ell}(\mathrm{t}-\Delta \mathrm{t})^{\circ}=\text { previous time step lower limit , } \\
& F_{u}(t) \quad=\quad \text { upper limit on flow blockage at time } t \\
& F_{u}(t-\Delta t)=\quad \text { previous time step upper limit } \\
& \overline{\mathrm{F}}_{\mathrm{u}}(\mathrm{t}) \quad=\quad \overline{\mathrm{F}}_{\mathrm{A}}(\mathrm{t})+1.7 \times \text { standard deviation of flow blockage } \\
& \text { data (found from upper dashed line in Figure } 83 . \\
& \mathrm{p}_{\mathrm{f}}(\mathrm{t})=\text { percentage of rods which fail in the time step } \mathrm{t}-\Delta \mathrm{t} \text { to } \mathrm{t} \\
& \bar{F}_{\ell}(t)=\quad \bar{F}_{A}(t)-1.0 \times \text { standard deviation (from lower dashed } \\
& \text { line in Figure 83). }
\end{aligned}
$$

(3) Sample Flow Blockage Problems. Three sample problems with different stress-temperature histories were run to demonstrate typical output of the FRAIL flow hlockage routines. Each problem is explained by three graphs:

(1) The stress-temperature history (along with the appropriate average rupture temperature from Figure 83)

(2) The percentage of failed rods as a function of the stresstemperature history

(3) The bcst cstimate of the blockage for a group of similar rods (with upper and lower bounds on the calculated blockage) as a function of the stress-temperature history. 
Figure 85 shows the flow blockage resulting when the stress is held constant. Rods begin failing as. soon as the lower temperature limit (first dashed line in Figure 85a) is exceeded. All rods are failed when the upper temperature limit (second dashed line) is reached. As pointed out in Section 3.1.3(1), the upper and lower temperature limits were chosen to include all available rupture data. The calculated flow blockage fraction (Figure $85 \mathrm{c}$ ) appears to give reasonable results. When all rods have failed, the best estimate flow blockage is just the average blockage as calculated in Equation (24). This is to be expected for a problem in which the stress is held constant. The difference between the upper and lower blockage limits increases with temperature. This implies that the best estimate flow blockage becomes more uncertain as the temperature increases.

Figure 86 shows the flow blockage resulting from a prohlem in which both temperature and stress are increasing with time. The flow blockage fraction increases until all the rods have failed. The final flow blockage value in this case is an accumulation of blockages as calculated by Equation (25).

Figure 87 is an example of the flow blockage calculated when about $50 \%$ of the fuel rods survive the transient. A temperature reversal stopped the rod failure trend. Although the stress continued to rise, flow blockage ceased to accumulate when the failure rate reached zero. 


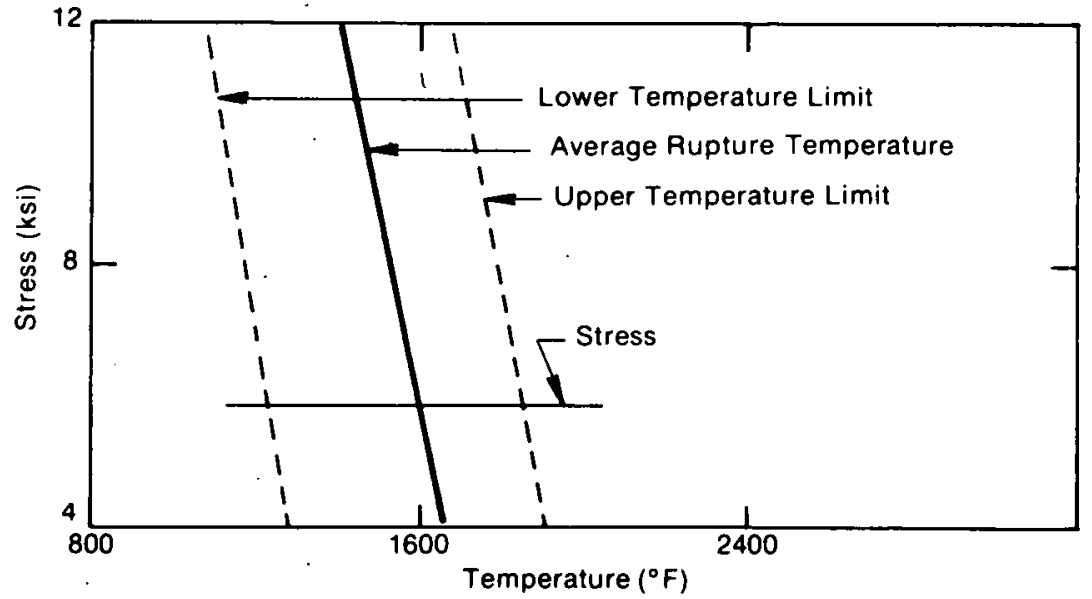

(a) Stress-Temperature History

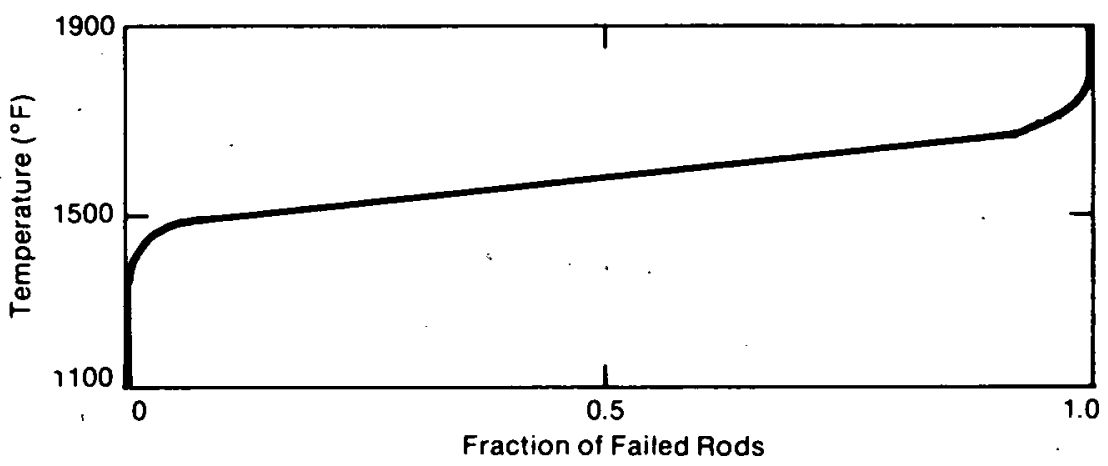

(b) Percentage of Failcd Rods as a Function of the Stress-Temperature History

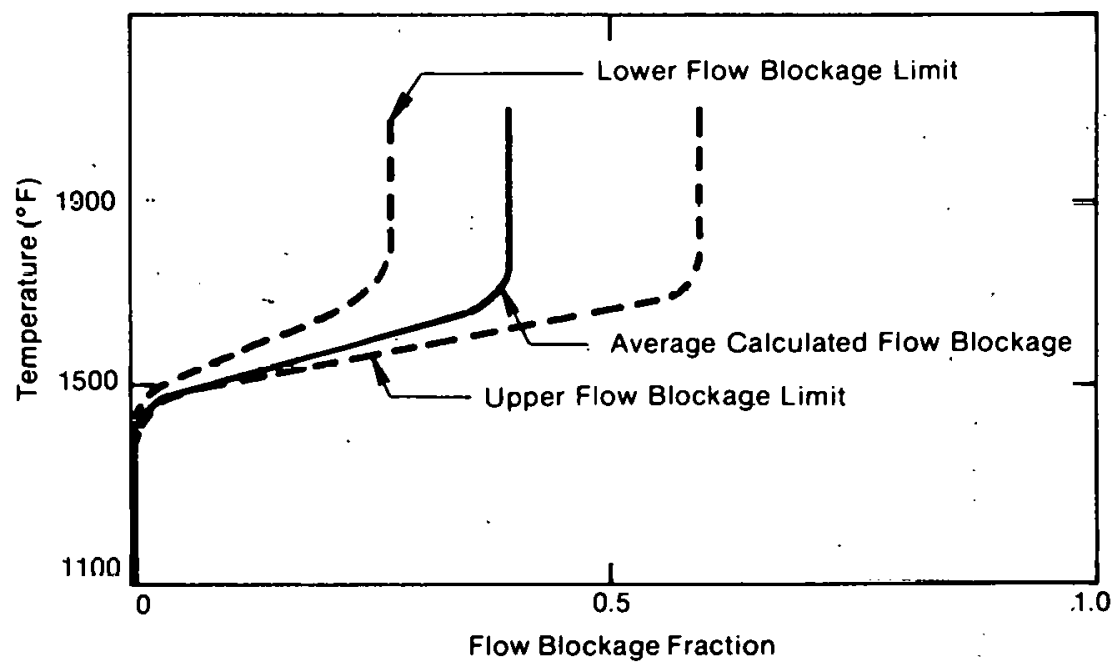

(c) Best Estimate of Blockage for a Group of Similar Rods as a Function of the Stress-Temperature History

INEL-A-1506

Fig. 85 Constant stress șample problem. 


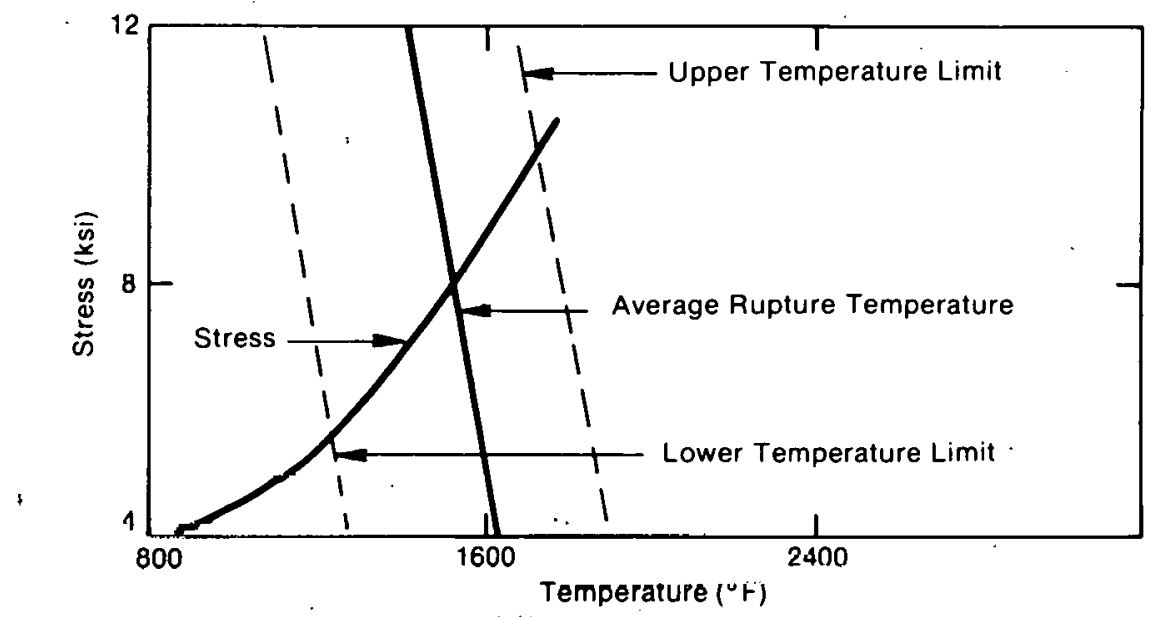

(a) Stress-Temperature History

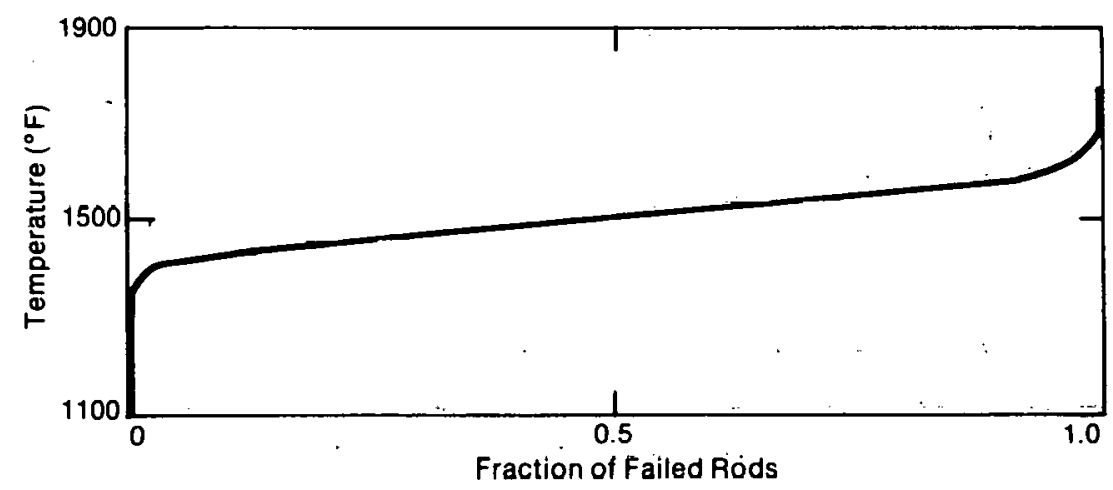

(b) Porcontago of Failod Rode ae a Function of tho Stroes-Tomporature Hiatory

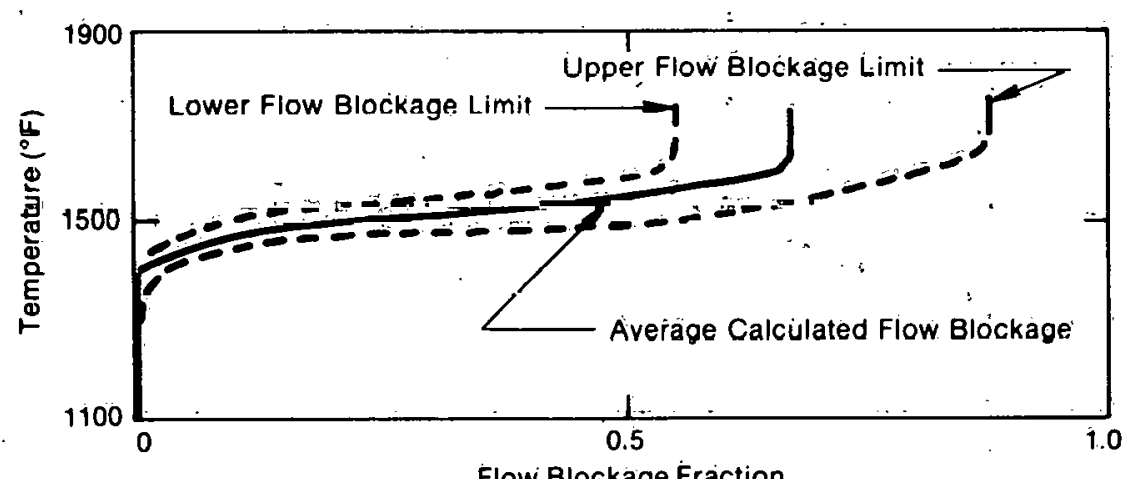

Flow Blockage Fraction

(c) Best Estimate of Blockage for a Group of Similar Rods as a Function of the Stress-Temperature History

INEL-A-1507

Fig. 86 Increasing temperature and stress sample problem. 


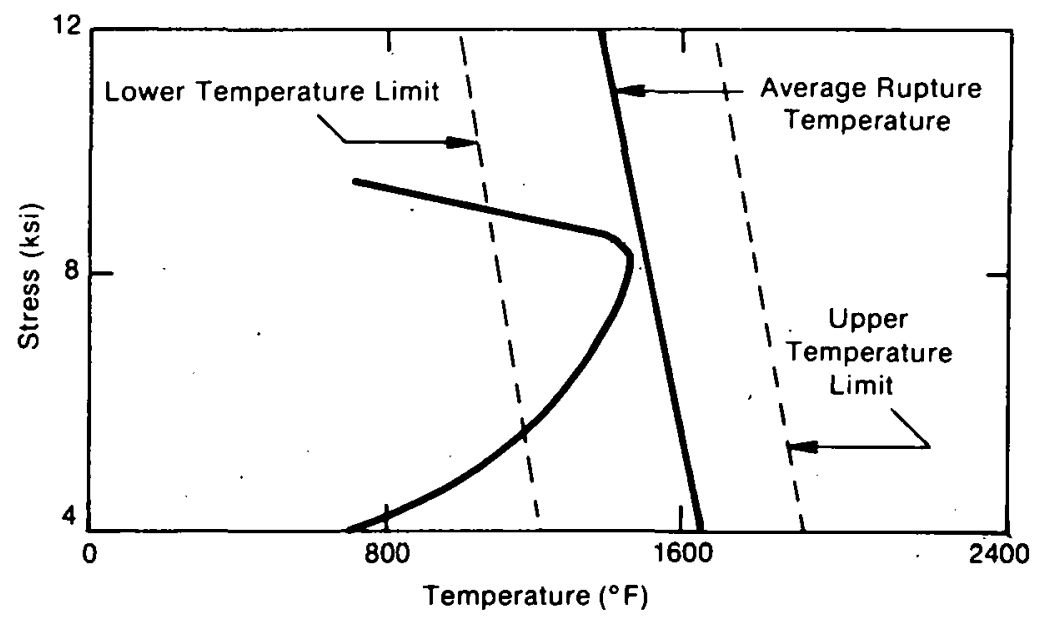

(a) Stress-Temperature History

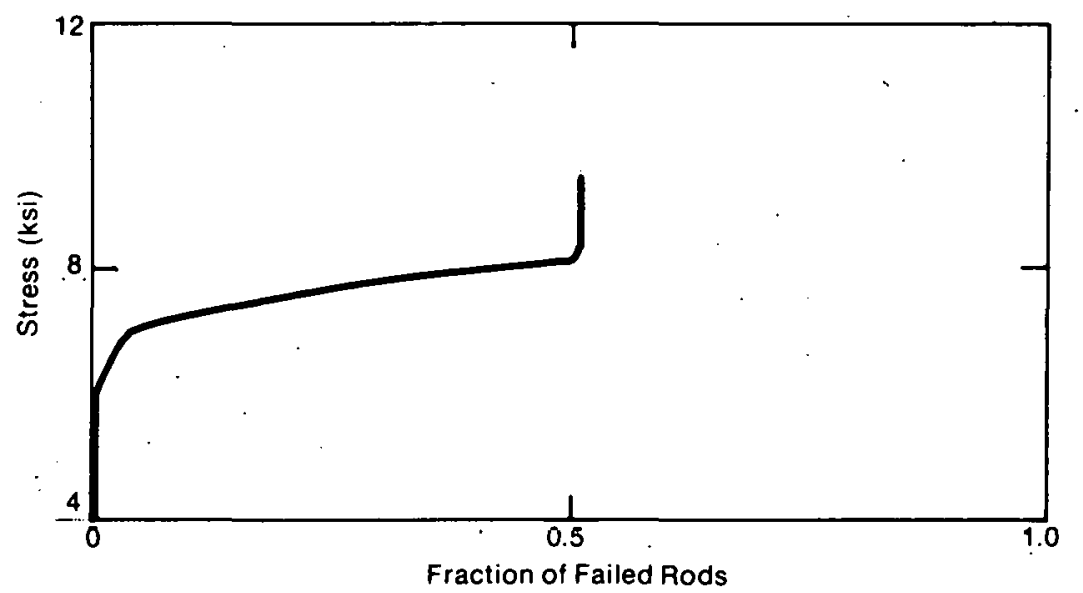

(b) Percentage of Failed Rods as a Function of the Stress-Temperature History

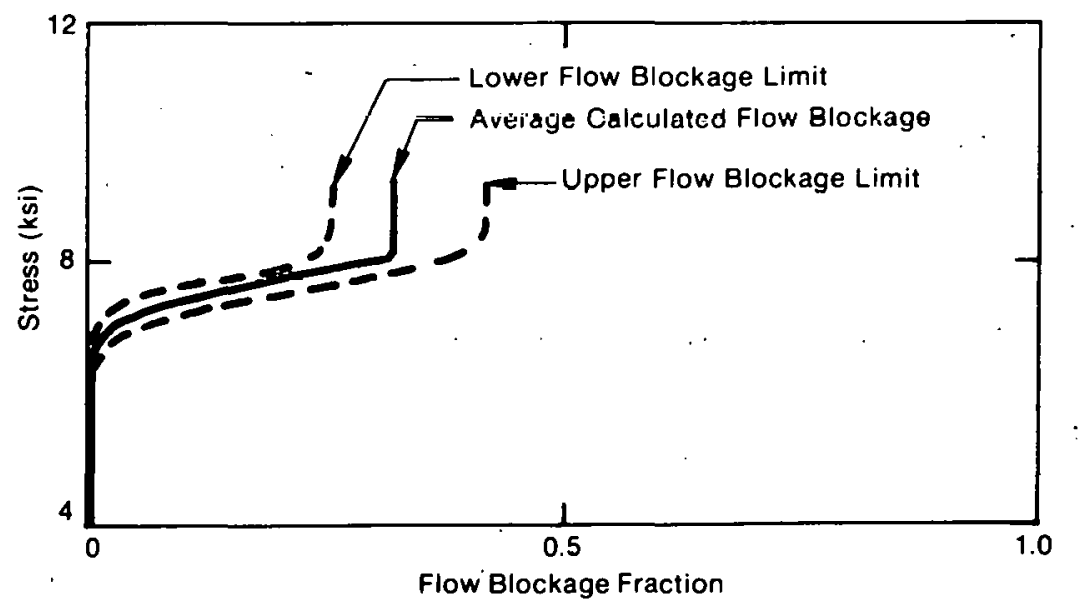

(c) Best Estimate of Blockage for a Group of Similar Rods as a Function of the Stress-Temperature History INEL-A-1508

Fig. 87 Tcmporature reversal sample problem. 


\section{IV: REACTOR BEHAVIOR PROGRAM}

R. R. Stiger, Manager

Accomplishments are reported for loss-of-coolant accident (LOCA) analysis development, containment analysis development, code verification, and a LOCA best estimate/ evaluation model (BE/EM) comparison:

\section{LOCA CODE DEVELOPMENT}

S. R. Fischer and L. V. Ellis

Analytical model development efforts have been directed toward finalizing a version of RELAP4 that includes best estimate models suitable for the simulation of the reflood portion of a pressurized water reactor (PWR) loss-of-coolant accident. Substantial changes and additions were made to enable RELAP4 to perform satisfactory best estimate reflood calculations for a PWR. About 7000 update cards, including some 20 new subroutines, were added to RELAP4/MOD.5. This development effort has culminated in RELAP4/MOD6 Version 1, which contains the MODS options plus the new PWR reflood models.

The new heat transfer and hydraulics models were debugged and checked out both individually and coupled. Prior to internal release, RELAP4/MOD6 Version 1 was subjected to preliminary developmental verification by comparing code calculations, with experimental

data. The code calculations for one blowdown and four reflood simulations showed reasonable agreement with the test data.

The following RELAP4/MOD,6 PWR reflood models are described:

(1) Moving heat conduction mesh

(?) Lorọ! mạss flux

(3). Implicit and explicịt entrainment

(4) Deentrainment

(5) Fallback

(6) Core superheat. 


\subsection{Moving Mesh Model}

The moving mesh model provides for detailed heat transfer calculations in the vicinity of a moving quench front or mixture level. User-defined coarse core heat slabs will be partitioned into a number of finer slabs when a quench front exists near the coarse slab. Use of this model will smooth the sudden increase in volume heat addition when a large coarse slab quenches. For a small break LOCA or during reflood, the model can be used to refine heat transfer calculations in the vicinity of a moving mixture level. Separate heat conduction calculations are made for each moving heat slab conductor using local fluid conditions to evaluate the surface heat transfer coefficient.

The moving mesh will initialize at the bottom of user-specified coarse heat slab stacks. The moving mesh model is sufficiently versatile so it can be employed simultaneously in different core stacks of coarse heat slabs such as in a hot, average, and cold core channel. Figure 88 indicates the heat slab partitioning employed in establishing a moving mesh for a coarse slab stack. The mesh moves in steps of fine mesh lengths. To simplify the calculation, the fine mesh length, DZF, should be input as an integral divisor of the medium mesh length, DZM, which should also be input as an integral divisor of the common length of the coarse slab making up the stack. At each time step during a transient, the moving mesh is positioned such that the location of the quench point $\left(\mathrm{T}_{\mathrm{wall}}-\mathrm{T}_{\text {sat }}=60^{\circ} \mathrm{F}\right)$ is near a point one-third the distance up the fine mesh group.

\subsection{Local Mass Flux Model}

The local mass flux model computes a local mass flux for use in the heat slab conductor heat transfer calculations when a mixture level exists within a volume. The model is used in conjunction with the local heat slab quality calculation. The model can be activated automatically by input cards when a mixture level exists within the volume. The mass flow rate of the coolant adjacent to the heat slabs above the mix ture level is set equal to the primary junction flow above the mixture level. The mass flow of the coolant adjacent to the heat slabs below the mixture level is set equal to the primary junction flow beneath the mixture level. A local mass flux, $\mathrm{G}_{\text {local }}$, is computed for the heat slab containing the mixture level as follows:

$$
G_{\text {local }}=\bar{\alpha}_{\text {mix }} G_{\text {top }}+\left(1-\bar{\alpha}_{\text {mix }}\right) G_{\text {bot }}
$$

where

$$
\begin{aligned}
& \bar{\alpha}_{\text {mix }}=\frac{x_{\text {local }} V_{g}}{\bar{v}_{\text {mix }}} \\
& \bar{v}_{\text {mix }}=\mathrm{X}_{\text {local }} \mathrm{V}_{\mathrm{g}}+\left(1-\mathrm{X}_{\text {local }}\right) \mathrm{V}_{\ell} \\
& \mathrm{G}_{\text {top }}=\text { the primary junction mass flux at the top of the volume } \\
& \mathrm{G}_{\text {bot }}=\text { the primary junction mass flux at the bottom of the volume }
\end{aligned}
$$




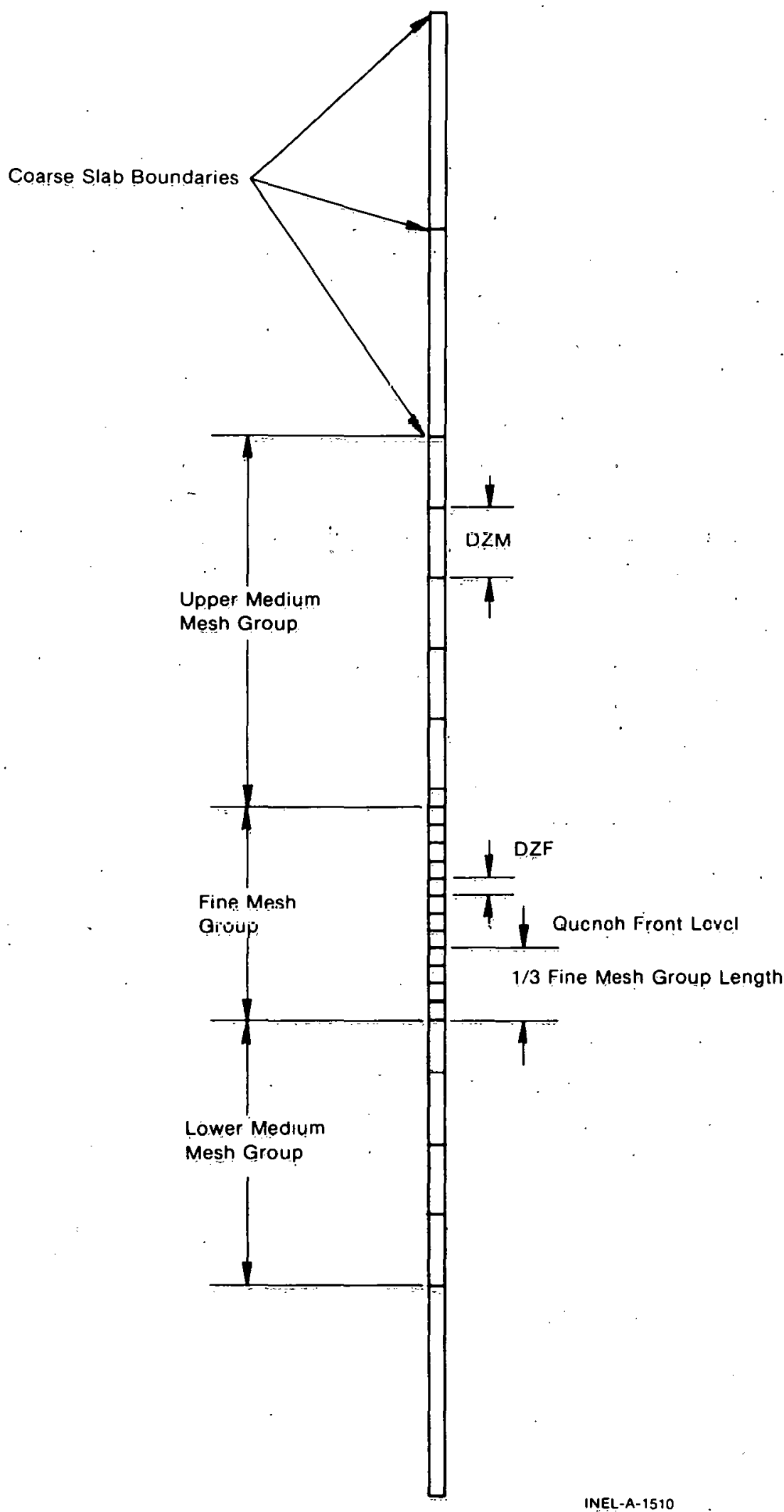

Fig. 88 Instantaneous moving mesh partitioning of coarse heat slab conductors. 


$$
\begin{array}{lll}
\mathrm{X}_{\text {local }} & = & \text { local heat slab quality } \\
\mathrm{V} & = & \text { saturated specific volume } \\
\ell & =\text { liquid } \\
\mathrm{g} & =\text { vapor } \\
\bar{\alpha}_{\text {mix }} & =\text { void fraction of mixture. }
\end{array}
$$

The local mass fluxes computed by this model are more representative of the true local condition than the volume average mass flow rate that would otherwise be used for all heat slabs within the volume.

\subsection{Implicit and Explicit Entrainment Models}

Entrainment phenomena that occur in the core during reflood can be computed using a number of options present in RELAP4/MOD6. The entrainment model not only provides fluid conditions for use in heat transfer calculations but also yields an appropriate core outlet mass flow. Two seperate models are available to implement these entrainment options. An explicit model used in RELAP4-FLOOD[63] or an implicit model based on a modified bubble rise concept can be used to simulate entrainment phenomena in RELAP4/MOD6.

Use of the explicit model involves setting the core outlet flow equal to that calculated explicitly from a given entrainment correlation. That is, the junction mass flow computed from the simultaneous solution of the conservation equations is overridden by the explicit entrainment calculation as

$$
W_{\text {out }}=E N \star W_{\text {in }}
$$

where

$$
\begin{array}{ll}
\mathrm{W}_{\text {out }}= & \text { mass flow rate out of core } \\
\mathrm{W}_{\text {in }}= & \text { mass flow rate into core } \\
\mathrm{EN} .= & \begin{array}{l}
\text { fraction of core inlet flow entrained (calculated from user-specified } \\
\text { entrainment correlation). }
\end{array}
\end{array}
$$

The implicit entrainment model involves calculating an appropriate core outlet junction density and quality based on an entrainment correlation. The implicit entrainment model employs a modified bubble rise model which allows a two-phase mixture to exist above a collapsed liquid layer as shown in Figure 89. Figure 89 indicates the possible physical characteristics of a two-phase core volume using the implicit entrainment option. The quality of the two-phase mixture is determined from an entrainment correlation as 


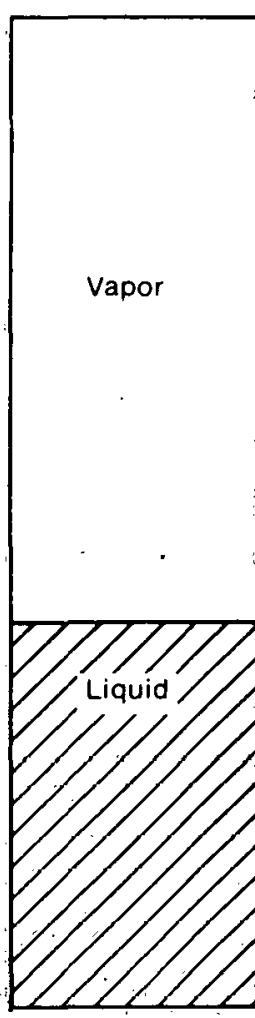

- No Entrainment

- Complete Phase Separation

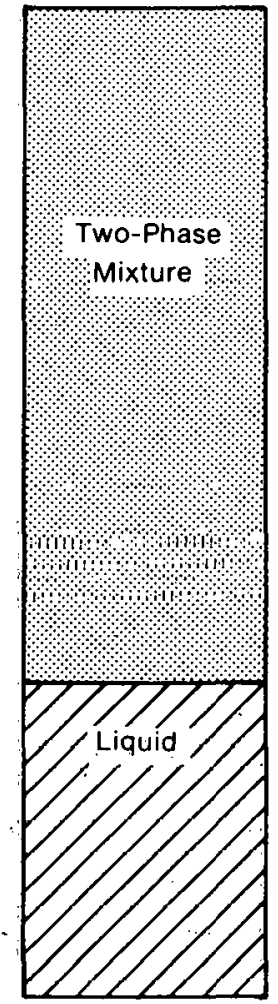

- Entrainment

- Collapsed Liquid Region

- llomogeneuis Tiwu Phase Mixture Above Liquid

\section{- Mixture Quality Determined from Entrainment Mưdel}

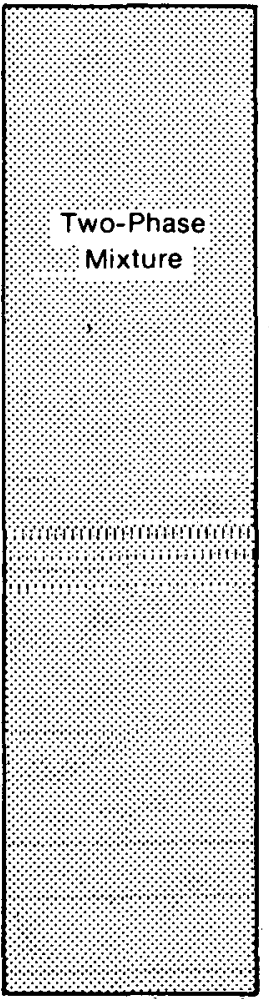

- Mạximum Entrainment

-Volume Homogeneous

where

$$
X_{\text {ábov }}=\frac{W_{g}}{W_{g}+E N * W_{i n}}
$$

$$
\begin{array}{ll}
\mathrm{X}_{\mathrm{abov}}= & \text { quality of mix ture above collapsed liquid level } \\
\mathrm{W}_{\mathrm{g}}= & \text { net vapor generation within collapsed liquid region } \\
\mathrm{EN}= & \text { entrainment fraction from user-specified correlation } \\
\mathrm{W}_{\mathrm{in}}= & \text { core inlet mass flow rate. }
\end{array}
$$


The pressure head terms in the momentum equation are adjusted to reflect the liquid redistribution within the fluid volume when the implicit entrainment model is used. The conservation equations are then used to compute the core outlet flow rate. The implicit model is compatible with the current implicit solution technique for solving the conservation equations and would appear to yield a more realistic model of the entrainment phenomena than the explicit model.

In addition to the entrainment correlations available in RELAP4-FLOOD, two new entrainment options have been added for use in the implicit mode. These options are the Steen-Wallis and REFLUX entrainment models. One entrainment model is based on an annular flow droplet entrainment correlation developed by Steen and Wallis $[64,65]$.

For fully turbulent flow, the critical superficial vapor velocity for the onset of entrainment is defined by

$$
\mathrm{J}_{\text {crit }}=\operatorname{HC2}\left(\frac{\rho_{\ell}}{\rho_{g}}\right)^{1 / 2}\left(\frac{\sigma}{\mu_{g}}\right)
$$

where

$$
\begin{array}{lll}
\rho_{\ell} & = & \text { liquid density }\left(1 \mathrm{bm} / \mathrm{ft}^{3}\right) \\
\rho_{\mathrm{g}} & =\quad \text { vapor density }\left(1 \mathrm{bm} / \mathrm{ft}^{3}\right) \\
\sigma & =\quad \text { surface tension }\left(1 \mathrm{~b}_{\mathrm{f}} / \mathrm{ft}\right) \\
\mu_{\mathrm{g}} & =\quad \text { vapor viscosity }\left(1 \mathrm{~b}_{\mathrm{f}}-\mathrm{s} / \mathrm{ft}\right) \\
\mathrm{HC} 2 & =\quad \text { user-input constant. }
\end{array}
$$

Entrainment commences when the volumetric flux of vapor generated by heat transfer to the collapsed liquid region exceeds the critical flux, $\mathrm{J}_{\mathrm{g}_{\text {crit }}}$.

Once entrainment is initiated, the fraction of core inlet liquid flow which is entrained is given by.

$$
E N=E N 2\left[1-e^{-H C l}\left(V_{g}^{*}-H C 2\right)\right]
$$

where

$$
\begin{aligned}
\mathrm{EN} 2 & =\text { user-input maximum entrainment fraction } \\
\mathrm{HC} 1 & =\text { user-input constant } \\
\mathrm{V}_{\mathrm{G}}^{*} & =\frac{\mathrm{J}_{\mathrm{g}} \mu_{\mathrm{g}}}{\sigma}\left(\frac{\rho_{\mathrm{g}}}{\rho_{\mathrm{l}}}\right)^{1 / 2}=\text { dimensionless vapor velocity } \\
\mathrm{J}_{\mathrm{g}} & =\text { volumetric flux of vapor based on core area }(\mathrm{ft} / \mathrm{s}) .
\end{aligned}
$$


The other entrainment option is somewhat similar to that employed by. Kirchner and Griffith in the REFLUX code[66]. This model can only be utilized in the implicit mode.

Application of the model at each time step proceeds by integrating the net heat flux into the collapsed liquid region, starting at the core bottom, until the net vapor generated exceeds a critical velocity given by

$$
v_{g_{\text {crit }}}=\frac{\rho_{\ell}}{\left(\rho_{\ell}-\rho_{g}\right)}\left(\frac{4 W_{e}}{3 C_{D}}\right)^{1 / 4}\left[\frac{\sigma g\left(\rho_{\ell}-\rho_{g}\right)}{\rho_{g}^{2}}\right]^{1 / 4}
$$

where

$$
\begin{aligned}
& \mathrm{We}_{\mathrm{e}}=\begin{array}{l}
\text { Weber number (Kirchmer's recommended value of } 7.5 \text { is } \\
\text { used) }
\end{array} \\
& \mathrm{C}_{\mathrm{D}}=\begin{array}{l}
\text { drag coefficient (Kirchner's recommended value of } 0.45 \text { is } \\
\text { used) }
\end{array} \\
& \mathrm{g}=\text { gravitational acceleration }\left(\mathrm{ft} / \mathrm{s}^{2}\right) .
\end{aligned}
$$

For the case when subcooled liquid enters the core, the elevation $\left(z_{\text {crit }}\right)$ in the core at which the integrated heat flux generates enough vapor to equal the critical value is given by

where

$$
\int_{0}^{z} \mathrm{crit} Q d z=w_{\text {in }}\left(h_{\ell}-h_{i n}\right)+v_{g_{\text {crit }}} \cdot A_{\text {core }} \dot{g h_{l g}}
$$

$$
\begin{aligned}
& \mathrm{Q}=\text { local heat flux }(\mathrm{Btu} / \mathrm{s}) \\
& \mathrm{z}=\text { vertical distance from bottom of core }(\mathrm{ft}) \\
& \mathrm{W}_{\text {in }}=\text { core inlet liquid flow rate }(\mathrm{lbm} / \mathrm{s}) \\
& \mathrm{h}_{\mathrm{in}}=\text { inlet liquid enthalpy }(\mathrm{Btu} / \mathrm{lbm}) \\
& \mathrm{h}_{\ell \mathrm{g}}=\text { latent heat of vaporization }(\mathrm{Btu} / \mathrm{lbm}) \\
& \mathrm{A}_{\text {core }}=\quad \text { core cross-sectional area }\left(\mathrm{ft}{ }^{2}\right) \\
& \mathrm{h}_{\ell}=\text { saturated liquid enthalpy }(\mathrm{Btu} / \mathrm{lbm}) .
\end{aligned}
$$

The quality above the liquid level is calculated by assuming that all liquid above $\mathrm{z}_{\text {crit }}$ is mixed homogeneously with the vapor generated within the collapsed liquid region. The two-phase mixture above a liquid region simulates the liquid distribution within the volume that would be expected during entrainment. 


\subsection{Deentrainment Model for Upper Plenum}

During reflood, liquid droplets that are entrained in the core will be transported into the upper plenum. The liquid drops will contact control rod guide tubes, support columns, and other structural members, which will act to deentrain the liquid. Therefore, a buildup of liquid at the core outlet may occur. In addition, vapor passing through the upper plenum will reentrain some of the deentrained liquid and carry the liquid out into the hot legs. The purpose of the deentrainment model is to compute the quality of the two-phase fluid flowing from the upper plenum into the hot legs. A correlation based in part on entrainment phenomena above sieve trays in distillation columns ${ }^{[67]}$ can be used to compute the amount of liquid entrained in the upper plenum. Then the modified bubble rise approach can be applied again to compute the various junction properties, and the solution of the mass conservation equation will yield the proper junction flows.

The correlation used to compute the net liquid entrained at the upper plenum hot leg junction is

$$
w_{\ell_{\text {ent }}}=(1-\varepsilon)\left[\left(\frac{Z_{\text {crit }}-Z}{Z_{\text {crit }}}\right) w_{l_{\text {in }}}+c_{1} v_{g}^{c_{2}}\left(\frac{Z}{z_{\text {crit }}}\right)\right]
$$

where

$$
\begin{aligned}
& \left(\frac{z_{\text {crit }}-z}{z_{\text {crit }}}\right)=0 \text { for } z \geq z_{\text {crit }} \\
& \left(\frac{Z}{z_{\text {crit }}}\right)=1 \text { for } z \geq z_{\text {crit }} .
\end{aligned}
$$

and

$$
\begin{array}{lll}
\mathrm{V}_{\mathrm{g}}= & \begin{array}{l}
\text { superficial vapor velocity in upper plenum (based on cross-sectional } \\
\text { area of upper plenum) }(\mathrm{ft} / \mathrm{s})
\end{array} \\
\mathrm{W}_{\ell_{\mathrm{ent}}}= & \begin{array}{l}
\text { liquid entrained (flow) above pool in upper plenum }(\mathrm{lbm} / \mathrm{s}) \\
\epsilon
\end{array} \quad \text { deentrainment efficiency (defined below) } \\
\mathrm{W}_{\ell_{\mathrm{in}}}= & \text { liquid (entrained) flow from core into upper plenum }(\mathrm{lbm} / \mathrm{s}) \\
\mathrm{Z} & =\quad \text { height of liquid pool in upper plenum }(\mathrm{ft}) \\
\mathrm{Z}_{\mathrm{crit}} & = & \text { user-defined critical pool depth }(\mathrm{ft}) \\
\mathrm{C}_{1}, \mathrm{C}_{2}= & \text { user-input constants }
\end{array}
$$


also

$$
\begin{gathered}
\varepsilon=\varepsilon_{0}+\left(\varepsilon_{1}-\varepsilon_{0}\right) e^{B\left(V_{g}-V_{c r i t}\right)} \text { for } V_{g}>V_{c r i t} \\
\varepsilon=\varepsilon_{1} \text { for } V_{g} \leq V_{c r i t}
\end{gathered}
$$

where

$$
\begin{aligned}
& \epsilon_{0}=\text { minimum deentrainment efficiency (user-input) } \\
& \epsilon_{1}=\text { maximum deentrainment efficiency (user-input) } \\
& \mathrm{V}_{\text {crit }}=\text { iser-input critical value of } \mathrm{V}_{\mathrm{g}}(\mathrm{ft} / \mathrm{s}) \\
& B=\text { user-input constant. }
\end{aligned}
$$

The mixture quality at the upper plenum/hot leg junction which will be used in the modified bubble rise model is calculated as follows

where

$$
x_{\text {abov }}=\frac{w_{g}}{w_{g}+w_{\ell \text { ent }}}
$$

$$
\mathrm{w}_{\mathbf{g}}=\quad \text { vapor mass flow rate from core into upper plenum }(\mathrm{lbm} / \mathrm{s}) \text {. }
$$

Other approaches to simulate deentrainment phenomena within the RELAP4 code are possible. These approaches include use of the Wilson bubble rise model $[68,69,70,71]$, use of the standard bubble rise model with constant bubble rise velocity, simulating complete phase separation using a large bubble rise velocity (total deentrainment), or use of the homogeneous model which simulates no deentrainment.

\subsection{Fallback Model for Core/Upper Plenum Junction}

The fallback model is closely tied to the upper plenum deentrainment model. The distribution of liquid in the upper plenum is governed by the core vapor velocity. As the core vapor generation diminishes and the liquid head in the upper plenum increases, the possibility of fallback of liquid into the core arises. This situation is compatible with the RELAP slip model, which allows countercurrent flow. The slip velocity can be limited in the high void fraction region by specifying a flooding correlation. The user can specify individual Wallis-type flooding correlations for various junctions.

The calculation of fallback proceeds by first determining the dimensionless volumetric flux of vapor passing through the upper plenum, $\mathrm{J}_{\mathbf{g}}^{*}$, based on the upper plenum area 


$$
\underset{g}{J^{*}}=\frac{W_{g}}{A \rho_{g}}\left(\frac{\rho_{g}}{\rho_{\ell}-\rho_{g}}\right)
$$

where

$$
\begin{aligned}
& \mathrm{W}_{\mathrm{g}}=\text { vapor mass flow rate at core/upper plenum junction }(\mathrm{lbm} / \mathrm{s}) \\
& \mathrm{A}=\text { flow area of upper plenum }\left(\mathrm{ft}^{2}\right)
\end{aligned}
$$

The Wallis flooding correlation ${ }^{[72]}$ is given by

$$
W C 2=\mathrm{J}_{\mathrm{g}}^{*} \mathrm{1}^{1 / 2}+W C 1 \mathrm{~J}_{\mathrm{f}}^{\star} 1 / 2
$$

where

$$
\begin{array}{lll}
\mathrm{J}_{\mathrm{g}}^{*} & =\begin{array}{l}
\text { dimensionless volumetric flux of vapor at the core/upper } \\
\text { plenum junction }
\end{array} \\
\mathrm{J}_{\mathrm{f}}^{*} & =\begin{array}{l}
\text { dimensionless volumetric flux of liquid at the core/upper } \\
\text { plenum junction }
\end{array} \\
\mathrm{WC1}, \mathrm{WC2}= & \text { Wallis flooding correlation constants (user-input). }
\end{array}
$$

This correlation relates dimensionless counter-current volumetric fluxes of liquid and vapor at the core/upper plenum junction and can be used to make the following decisions regarding fallback:

If $\mathrm{J}_{\mathrm{g}}^{*}$ is greater than $\mathrm{J}_{\mathrm{g}_{\text {crit }}}^{*}=\mathrm{WC} 2$, then no fallback is possible. If $\mathrm{J}_{\mathrm{g}}^{*}<0.0$, then no adjustment of the junction liquid flow is made. For $0.0 \leqslant \mathrm{~J}_{\mathrm{g}}^{*} \leqslant \mathrm{WC2}$, flooding is assumed at the core/upper plenum junction, and the junction volumetric liquid flow is recomputed using the Wallis flooding correlation. The junction slip velocity is set equal to the smaller of the value computed from the user slip correlation or the value computed from a simultaneous solution of the conservation of mass equation with the Wallis correlation.

\subsection{Core Superheat Model for Reflood}

In order to improve the calculation of the surface heat transfer coefficient for both the moving mesh and coarse heat slab conductors, more accurate estimates of the local fluid properties can be calculated using the core superheat model. The superheat calculation begins by assigning a pseudovolume to each moving mesh or coarse heat slab. A steady state heat and mass balance is performed for each pseudovolume in the core region above the collapsed liquid level. A local fluid quality, $\mathrm{X}_{\text {local }}$; a local vapor temperature, $\mathrm{T}_{\mathrm{g}_{\text {local }}}$; and a local mass flux, $G_{\text {local }}$, are computed for each slab. Liquid is assumed to be at saturation conditions throughout the core. However, vapor superheating is calculated locally. At the core outlet, a superheated vapor enthalpy is calculated and then used in the convection term in the energy equation. 
The model is based on the following assumptions:

(1) The mass flow rate above the collapsed liquid level is assumed to be constant during each time step

(2) Potential and kinetic energy terms in the energy equation are neglected

(3) An energy partition function is defined to specify the fraction of energy leaving the heat slab that is transferred to each phase

(4) The entrainment model is used to specify the local liquid and vapor mass flow rates at the interface between the collapsed liquid and steam dome.

The local vapor and liquid mass flow rates at the point of entrainment are

$$
w_{g}=\frac{\int_{0}^{Z_{m}} Q d z-W_{i n}\left(h_{\ell s a t}-h_{i n}\right)}{h_{\ell g}}
$$

and

$$
W_{\ell \text { ent }}=E N * W_{\text {in }}
$$

where

$$
\begin{aligned}
& z=\text { vertical distance from core inlet (ft) } \\
& \mathrm{W}_{\mathrm{g}}=\text { local vapor mass flow rate at point of initiation of entrainment }(\mathrm{lbm} / \mathrm{s}) \\
& \mathrm{W}_{\ell_{\mathrm{ent}}}=\text { local entrained liquid mass flow rate at point of initiation of } \\
& \text { entrainment }(\mathrm{lbm} / \mathrm{s}) \\
& 7_{\mathrm{m}}=\text { hẹght in conre at which entrainment is initiated (ft) } \\
& \mathrm{Q}=\text { local heat transfer rate }(\mathrm{Btu} / \mathrm{s}-\mathrm{ft}) \\
& \mathrm{W}_{\mathrm{in}}=\text { liquid mass flow rate at core inlet }(\mathrm{lbm} / \mathrm{s}) \text {. } \\
& \mathrm{h}_{\ell_{\text {sat }}}=\quad \text { saturated liquid enthalpy }(\mathrm{Btu} / \mathrm{s}) \\
& \mathrm{h}_{\mathrm{in}}=\quad \text { enthalpy of liquid entering core }(\mathrm{Btu} / \mathrm{lbm}) \\
& \mathrm{h}_{\ell_{\mathrm{g}}}=\text { latent heat of vaporization }(\mathrm{Btu} / \mathrm{lbm}) \\
& \text { EN = entrainment fraction from user-specified correlation. }
\end{aligned}
$$


For each pseudovolume assigned to a heat slab above the mixture level, energy and mass balances are computed as follows. The mass balance is

$$
\begin{aligned}
& w_{\ell_{\text {out }}}=w_{\ell_{\text {in }}}-\frac{\lambda Q}{h_{\ell g}} \\
& w_{g_{\text {out }}}=w_{g_{\text {in }}}+\frac{\lambda Q}{h_{\ell g}}
\end{aligned}
$$

where

$$
\begin{array}{ll}
\lambda= & \begin{array}{l}
\text { energy partition function }=\text { fraction of total heat flux, } \\
\text { liquid phase }
\end{array} \\
\mathrm{W}_{\ell_{\text {out }}}= & \text { liquid mass flow rate out of pseudovolume }(\mathrm{lbm} / \mathrm{s}) \\
\mathrm{W}_{\ell_{\text {in }}}= & \text { liquid mass flow rate into pseudovolume }(\mathrm{lbm} / \mathrm{s}) \\
\mathrm{W}_{\mathrm{g}_{\text {out }}}= & \text { vapor mass flow rate out of pseudovolume }(\mathrm{lbm} / \mathrm{s}) \\
\mathrm{W}_{\mathrm{g}_{\text {in }}}= & \text { vapor mass flow rate into pseudovolume }(\mathrm{lbm} / \mathrm{s}) .
\end{array}
$$

The overall energy balance is

$$
W_{\ell_{\text {in }}} h_{\ell_{\text {sat }}}+W_{g_{\text {in }}} h_{g_{\text {in }}}+Q=W_{g_{\text {out }}} h_{g_{\text {out }}}+w_{\ell_{\text {out }}} h_{\ell_{\text {sat }}} .
$$

Solving for $\mathrm{h}_{\mathrm{g}_{\text {out }}}$ :

$$
h_{g_{\text {out }}}=Q+\frac{\left(w_{\ell_{\text {in }}}-w_{l_{\text {out }}}\right) h_{l_{\text {sat }}}+w_{g_{\text {in }}} h_{g_{\text {in }}}}{w_{g_{\text {out }}}}
$$

and equating this to

$$
h_{g_{\text {out }}}=h_{g_{\text {sat }}}+c_{p}\left(T_{\text {out }}-T_{\text {sat }}\right)
$$

where

$$
\begin{array}{ll}
\mathrm{h}_{\mathrm{g}_{\text {out }}}= & \text { vapor enthalpy at outlet of pseudovolume }(\mathrm{Btu} / \mathrm{lbm}) \\
\mathrm{h}_{\mathrm{g}_{\text {in }}}= & \text { vapur enthalpy at inlet of pseudovolumc }(\mathrm{Btu} / \mathrm{lbm}) \\
\mathrm{T}_{\text {out }}= & \text { vapor temperature at outlet of pseudovolume }\left({ }^{\circ} \mathrm{F}\right) \\
\mathrm{T}_{\text {sat }}= & \text { saturation temperature at outlet of pseudovolume }\left({ }^{\circ} \mathrm{F}\right) \\
\mathrm{T} & =\text { temperature }\left({ }^{\circ} \mathrm{F}\right)
\end{array}
$$




$$
\begin{aligned}
& \mathrm{C}_{\mathrm{p}}=\quad \text { vapor specific heat } \\
& \mathrm{h}_{\mathrm{g}_{\mathrm{sat}}}=\quad \text { saturated vapor enthalpy }(\mathrm{Btu} / \mathrm{lbm})
\end{aligned}
$$

yields the vapor temperature, $\mathrm{T}_{\text {out }}$, at the outlet of the pseudovolume.

In performing an energy and mass balance for a pseudovolume, certain restrictions must be placed on the vapor temperature. These are

and

$$
\text { if } Q>0 \text { and } T_{g_{\text {in }}}<T_{\text {wall }} \text {, then } T_{g_{\text {out }}} \leq T_{\text {wall }}
$$

$$
\text { if } Q<0 \text { and } T_{g_{\text {in }}}>T_{\text {wal }} \text {, then } T_{q_{\text {out }}} \geq T_{\text {wal }}
$$

where

$$
\begin{aligned}
& \mathrm{T}_{\mathrm{Bin}_{\mathrm{in}}}=\quad \text { vapor temperature at inlot to pocudovolume (IIF) } \\
& \left.\mathrm{T}_{\mathrm{g}_{\mathrm{out}}}=\quad \text { vapor temperature at outlet to pseudovolume ( }{ }^{\circ} \mathrm{F}\right) \\
& \mathrm{T}_{\text {wall }}=\quad \text { surface temperature of heat slab }\left(^{\circ} \mathrm{F}\right) \\
& \mathrm{Q}=\text { local heat flux, positive flux is from heat slab to fluid (Btu/s). }
\end{aligned}
$$

An additional assumption is necessary to handle reverse flow at the core inlet and to handle the situation when no liquid is present in core. A positive core vapor flow is assumed at all times. This is accomplished by setting the lower limit on core vapor flow to be a small positive mass flux of $20 \mathrm{lb} / \mathrm{h}-\mathrm{ft}^{2}$.

The local fluid properties for a heat slab can be computed once the above information is obtained:

$$
\begin{aligned}
& x_{\text {local }}=\frac{w_{q_{\text {in }}}+w_{q_{\text {out }}}}{w_{g_{\text {in }}}+w_{g_{\text {out }}}+w_{\ell_{\text {in }}}+w_{\ell}} \\
& T_{g_{\text {local }}}=\frac{T_{g_{\text {in }}}+T_{g_{\text {out }}}}{2} \\
& G_{\text {local }}=\frac{W_{g_{\text {in }}}+w_{g_{\text {out }}}}{A}
\end{aligned}
$$

where
$A=$ core flow area $\left(\mathrm{ft}^{2}\right)$.
$\mathrm{G}_{\text {local }}$ is a constant for all slabs above the liquid level. 


\title{
2. CONTAINMENT ANALYSIS DEVELOPMENT
}

\author{
R. A. Wells, J. F. Lime, S. W. James
}

The primary emphasis in the containment analysis development program is on development of the BEACON code for use in investigating reactor containment phenomena such as mixing of steam, water and air; two-phase, two-component flow between compartments; and water droplet deentrainment. The coupling of two-dimensional and one-dimensional Eulerian regions, modeling for lumped parameter regions, and coupling of these regions to one-dimensional regions has been reported $[6,13]$.

The ability to change flow areas at the point of coupling of Eulerian regions was developed, coded, and tested to provide the BEACON code with the capability of adequately modelling multicompartment containment system geometry. The continuing development effort will add interphase mass transfer, wall heat transfer, and other options to increase the usefulness of the code.

The latest version of the BEACON code, containing all of the capabilities developed to date, has been designated as BEACON/MOD1. A series of test cases run with BEACON/MOD1 indicate that the code is performing as desired and represents a useful tool for the analysis of containment systems.

\subsection{Unequal Area Coupling Technique}

The coupling of one-dimensional regions with different cross-sectional flow areas (unequal area coupling) is obtained by modifying the basic fluid computation equations to include multipliers that reflect the change in flow area and control volume across an unequal area coupling boundary. These multipliers are applied in a manner so as to decrease or increase a convective flux quantity across the computational cell control volume boundary. They are also used in adjusting cell-centered velocity values that are computed for interphase drag calculations.

For equal area coupling computations, the unequal area coupling multipliers are defaulted to a value of 1.0. At an unequal area boundary, input cell dimensions of each region are used to recompute the applicable multipliers to reflect the change in flow area and control volume across the boundary.

For computations of cell-centered variables, the computation control volume aligns with the cell edges and only the cell-edge area multipliers need to be applied in the equations. For the momentum flux computations, the computation control volume is centered on a cell edge and extends into the next cell. This requires a different set of area multipliers and introduces a control volume multiplier for each of the momentum flux iterations. 


\subsection{Test Cases}

The configuration shown in Figure 90 was used to test the unequal area coupling technique. The lumped parameter regions are large enough to be assumed infinite and are connected by two one-dimensional regions in series. Lumped Parameter Region 1 is initially at a pressure of $30 \mathrm{psia}$; the remaining regions have an initial pressure of $20 \mathrm{psia}$. The initial temperature of the system is $700^{\circ} \mathrm{R}$, and the void fraction is 0.9 . The area ratio at the coupling boundary is $2: 1$.

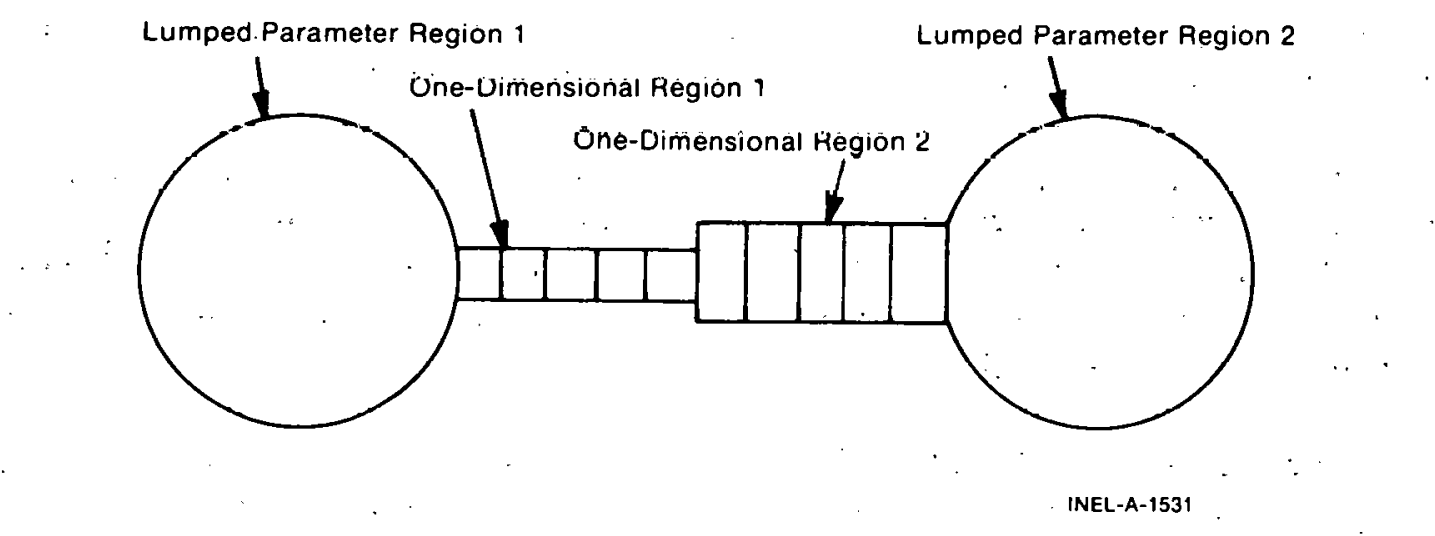

Fig. 90 Configuration for test case.

Steady state is essentially reached at $0.5 \mathrm{~s}$. Figure 91 shows the velocity and pressure distributions along the duct at steady state. The velocity of the vapor changes abruptly at the location of the area change and, also, as expected, the inertia of the droplets resists the sudden velocity change induced by a sudden expansion. This indicates that the unequal area coupling modifications are producing correct results.

Other test cases were run with the test configuration shown in Figure 90 modified by:

(1) Rotating the configuration $180^{\circ}$ so the high pressure lumped region and smaller one-dimensional region is on the right side.

(2) Rotating the configuration $90^{\circ}$ and $270^{\circ}$ so the configuration is in a vertical plane. (The gravitational force was set to zero.)

(3) C'hanging the vertıcally onented case to an axisymmetric coordinate system.

In all cases, numerical results were identical. 

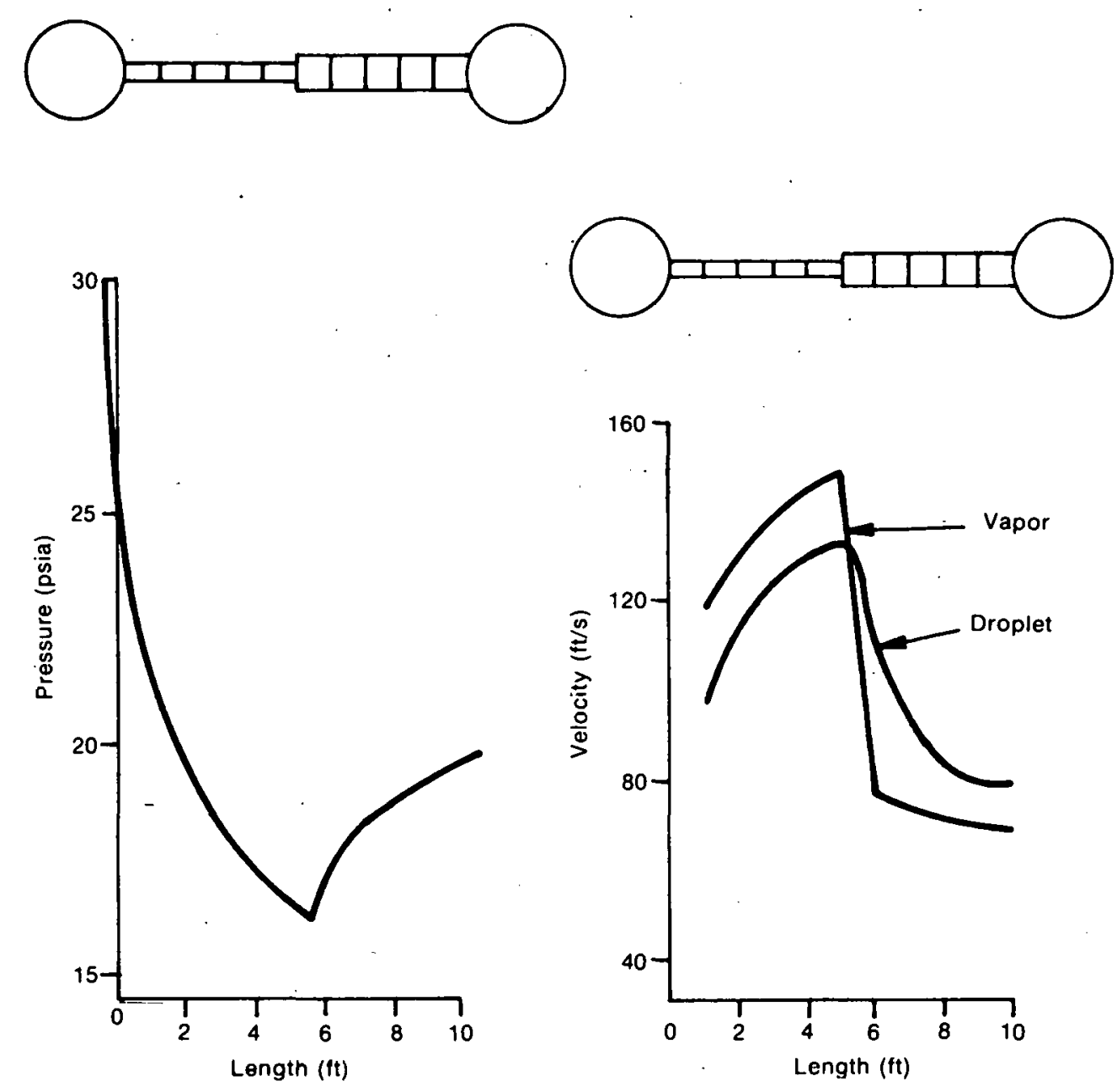

Fig. 91 Steady state pressure and velocity for test case.

\section{CODE VERIFICATTION}

R. E. Rice

A study was completed to investigate the sensitivity of the response surface uncertainty analysis technique to various unknowns in the analysis. This study is part of a continuing astivity to develop methods for determining the uncertainty of LOCA computer codes.

An initial study was completed to evaluate the RELAP4/MOD5 code with new data obtained from the pressurized water reactor blowdown heat transfer (PWR-BDHT) program being conducted at the Oak Ridge National Laboratory. In addition, a sensitivity study was comploted to svaluate potential improvements in the model. 
A prediction of the first boiling water reactor blowdown/emergency core cooling (BWR-BD/ECC) test was made with a version of the RELAP4/MOD5 code. This experiment will be conducted by the General Electric Company (GE) in a modified version of the two-loop test apparatus (TLTA). The prediction of the first BWR-BD/ECC test was compared with a RELAP4 analysis of the TLTA reference test.

Key findings of these studies are provided in the following sections.

\subsection{Sensitivity Study of the Response Surface Method of Uncertainty Analysis \\ N. D. Cox}

A sensitivity study of the response surface method of unccrtainty analysis was conducted using results from a LOCA analytical model. This study was part of a continuing effort to develop methods for evaluation of the uncertainty in PWR LOCA analytical models. As such, this study was concerned with the sensitivity of the output uncertainty density of peak cladding temperature to assumed changes in the input uncertainty densities, to the number of terms in the response surface equation, to the use of a different number of responses to evaluate the response surface equation, and to the use of a Gaussian density for representing the computed peak cladding temperature uncertainty. This work was approached through the use of the results obtained for an uncertainty analysis of a simplified 12-volume RELAP4/MOD5 model of the Trojan PWR $[6,47]$. The major results of this study follow.

3.1.1 Effect of Alternate Uncertainty Density Functions. When using alternate forms of uncertainty density. functions, the greatest effect on the peak cladding temperature density function resulted from the substitutions of densities with unlimited range for the uniform densities, which have limited ranges. The largest of these effects was comparable to that of changing the standard deviations of all the uncertainty densities by $20 \%$. This moderately large magnitude of change was partly because the most influential variable, the discharge coefficient at the side of the inlet pipe break nearest the vessel, was one of the two variables with uniform distributions. This result showed that the uniform density function should be used only when all allowed events are truly equiprohahle.

Figure 92 illustrates the effect on peak cladding temperature density caused by changing the input uncertainty densities for the discharge coefficient for the vessel side of the inlet pipe break and fuel gap conductivity from uniform, as in base case $\operatorname{RSM}(30.5)$, to Gaussian. The differences between these curves were comparable to those arising when the standard deviations of the input uncertainty densities were changed by $10 \%$.

The substitution of alternate density functions for those other than the uniform input densities resulted in changes comparable to or less than those induced by $10 \%$ changes in input standard deviations and, thus, were not significant.

3.1.2 Sensitivity to Distributional Spread. The greatest sensitivity of the response surface method, as applied to the 12-volume model, was to the standard deviations of the input uncertainty densities. The sensitivity of the peak cladding temperature density to 


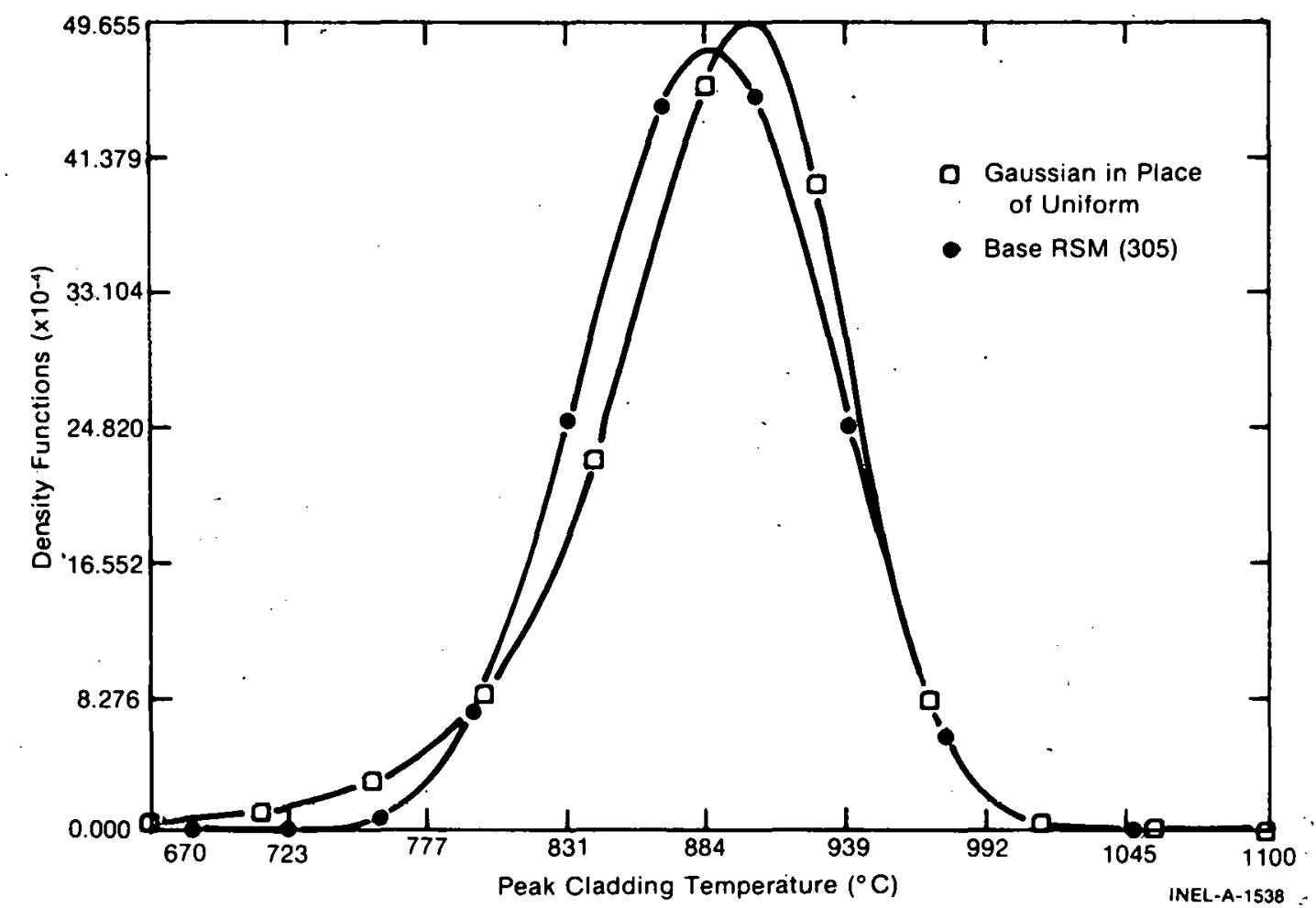

Fig. 92 Effect on peak cladding temperature density caused by changing input uncertainty densities for discharge coefficient for vessel side of inlet pipe break and fuel gap conductivity from uniform to Gaussian.

changes in the standard deviations of all of the input parameters is shown in Figure 93. This sensitivity to distributional specifications need not be a deterrent to the use of the technique for studying parameter margin. For example, with all the variations taken in this study, the probability of a peak cladding temperature greater than $1066^{\circ} \mathrm{C}\left(1950^{\circ} \mathrm{F}\right)$ was always less than 0.0001 .

3.1.3 Sensitivity to Lack-of-Fit. Densities for peak cladding temperature obtained using (a) varying numbers of terms in response surface equation and (b) different numbers of responses for evaluating the equation were compared with the base case results. The differences between cases were not significant.

3.1.4 Adequacy of the Gaussian Approximation for Computed Peak Cladding Temperature Density. The differences between the base case peak cladding temperature density and a Gaussian approximation were comparable to those generated by $10 \%$ changes in standard deviations and, thus, werc not significant.

\subsection{RELAP4/MOD5 Comparison with ORNL PWR-BDHT Test 104}

C. B. Davis

A comparison of calculations from a RELAP4/MOD5 Update $2^{[73]}$ core model and data from the PWR-BDHT separate effects program [74] (PWR-BDHT Test 104) was completed. This data comparison was conducted as part of developmental verification of RELAP4 to (a) assess the adequacy of current models to calculate the blowdown 


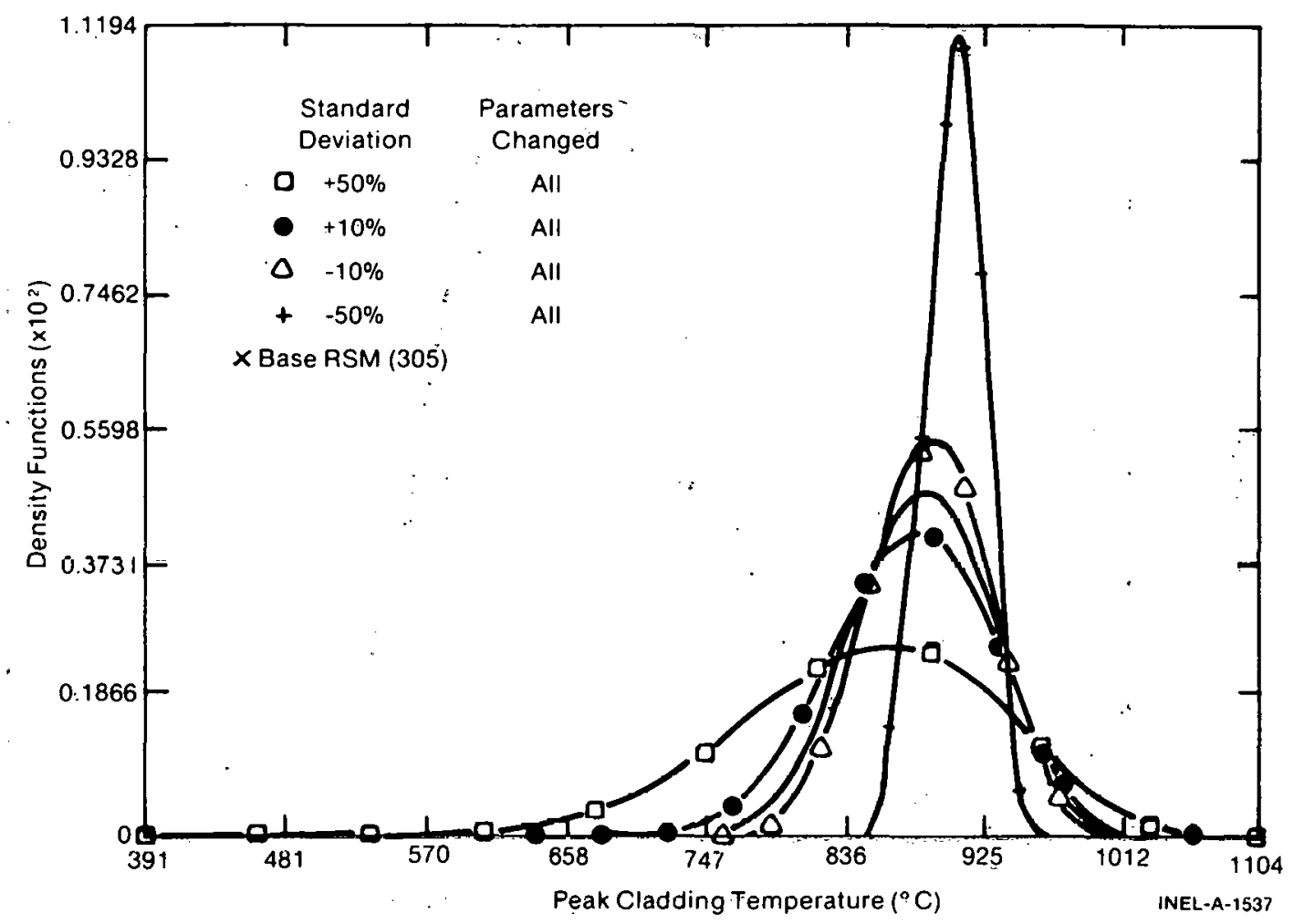

Fig. 93 Effect on peak cladding temperature density by changing standard deviations of input uncertainty densities.

thermal-hydraulic performance of a simulated reactor core and (b) provide a reference for future versions of the code. Measured flow and pressure histories were used as boundary conditions for the RELAP4 core model.

Figure 94 compares the calculated and measured cladding temperatures of the heater rod at the hot spot. At the hot spot, critical heat flux (CHF) was calculated and generally observed at approximately $0.3 \mathrm{~s}$ after rupture. (One of the rods experienced CHF at approximately $2 \mathrm{~s}$.) Measured hot spot cladding temperatures for the rods experiencing early $\mathrm{CHF}$ ranged from 1180 to $1230^{\circ} \mathrm{F}$, while the calculated peak clad temperature was $1220^{\circ} \mathrm{F}$. Within $2 \mathrm{~s}$ after the peak clad temperature was attained in the test, the rods at the hot spot apparently rewet and cooled down very rapidly. RELAP4 did not calculate the rewet and consequently overpredicted clad temperatures at the hot spot by as much as $350^{\circ} \mathrm{F}$ during the cooldown phase of the blowdown.

Sensitivity studies were conducted to investigate potential improvements in the model. The peak clad temperature at the hot spot (Figure 95) was calculated more accurately by RELAP4/MOD5 Update 2 with the Groeneveld 5.9 film boiling correlation than with the Dougall-Rohsenow correlation.

The use of the RELAP4 phase slip option in the core provided a small improvement in the calculated cladding temperature history at the hot spot. 


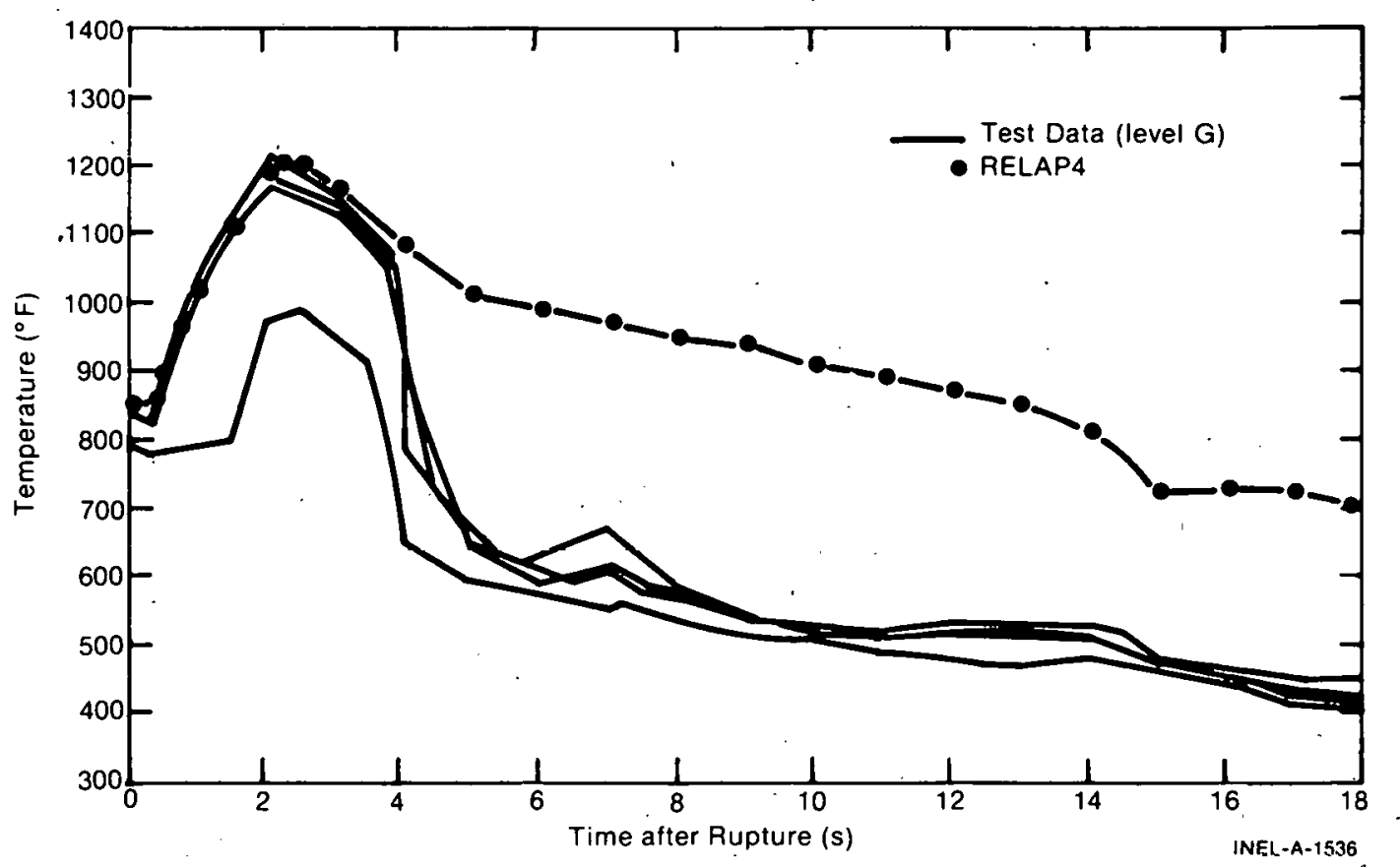

Fig. 94 Comparison of calculated and measured cladding temperatures at hot spot.

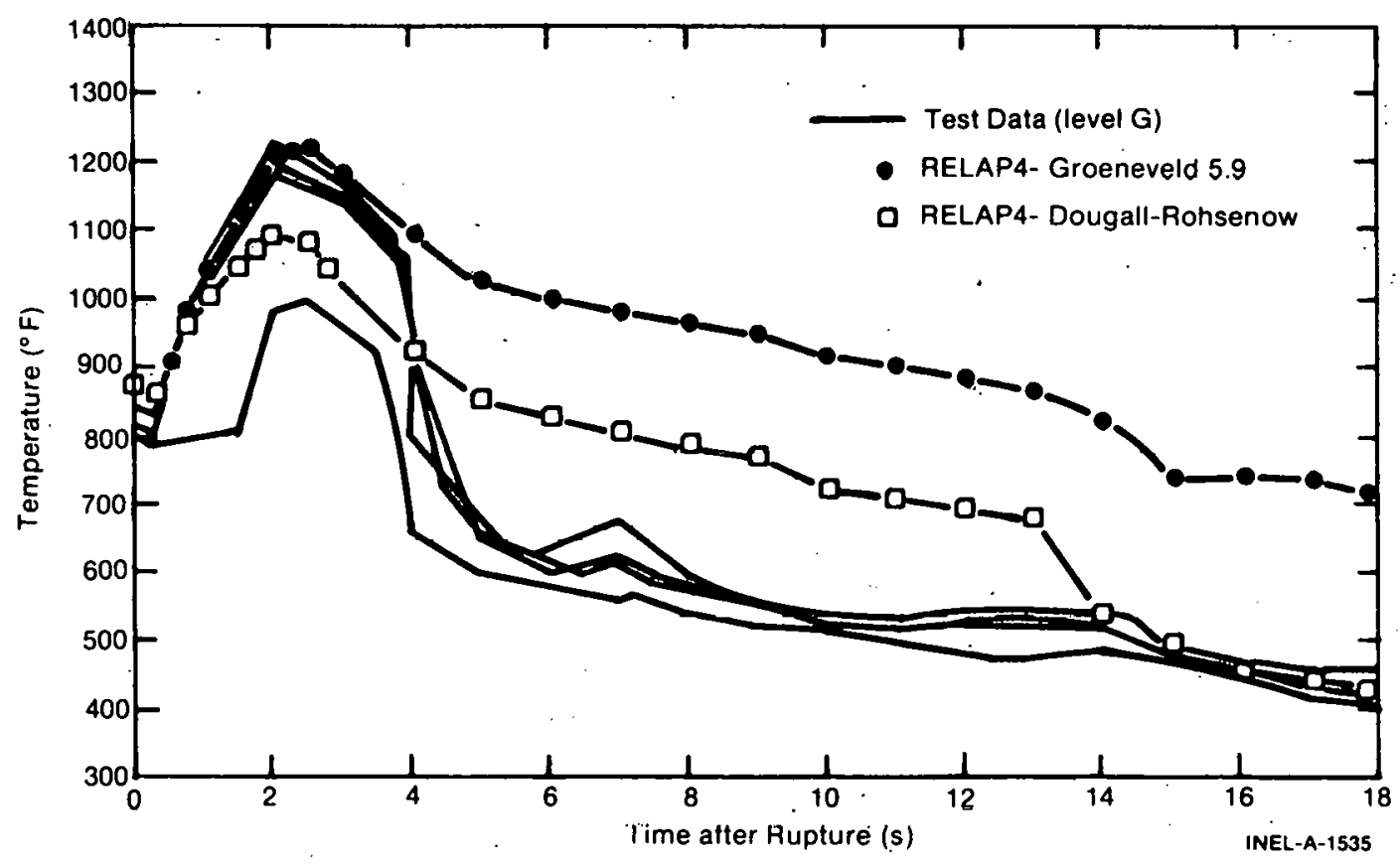

Fig. 95 Cladding temperature at hot spot. 
3.3 The First BWR-BD/ECC Test: A RELAP4 Prediction and Comparison with a RELAP4 Analysis of the TLTA BDHT Program Reference Test

R. R. Schultz

A RELAP4[a] prediction of the first experiment (TLTA-2 Test 1) in the BWRblowdown/emergency core cooling program $[75]$ is presented. This experimental configuration is the first step in changing the two-loop test apparatus at General Electric Company from a blowdown experimental apparatus to an emergency core cooling experimental apparatus. The major difference between this and previous tests is the replacement of the $7 \times 7$ heater rod bundle with an $8 \times 8$ bundle. The TLTA-2 test prediction is compared with the analysis of data from the BDHT program reference test (TLTA-1 Test 4903, Run 16) ${ }^{[13]}$ to assess the thermal-hydraulic differences. The initial core power for this comparison corresponds to an average bundle in a Browns-Ferry class BWR.

3.3.1 RELAP4 Model. The model utilized for the analysis of TLTA-2 'Test 1 data is basically the same as that used for the analysis of the data from TLTA-1 Test 4903, Run 16. Only those model changes necessary to match the revised TLTA-2 geometry were made. The Reference 13 core model was changed to match the $8 \times 8$ core configuration in the TLTA-2, and the upper plenum and core inlet volumes were changed.

3.3.2 Summary of Results. The TLTA-2 Test 1 prediction and the comparison of this prediction with the analysis of data from TLTA-1 Test 4903, Run 16 are summarized in curves, showing the system depressurization, the core hydrodynamics, and core heatup characteristics.

(1) System Pressure Response. The calculated steam dome pressure responses of the TLTA-2 Test 1 prediction and data-from TLTA-1 Test 4903, Run 16 analysis are shown in Figure 96. The steam dome pressure response is typical of all system pressure behavior.

Figure 96 shows the steam dome pressure calculated for the $8 \times 8$ bundle test to be dropping rapidly during the tirst $2 \mathrm{~s}$ atter the break. The pressure stabilizes after $2 \mathrm{~s}$ until a rapid depressurization occurs at $7.8 \mathrm{~s}$, when the jet pump suction uncovers. The most significant system pressure change occurs at $10.1 \mathrm{~s}$ as the recirculation pump suction line uncovers and results in lower plenum flashing at $10.3 \mathrm{~s}$. The steam dome pressure response tor the $\% \mathrm{x} \%$ bundle test is similar. Comparison of the two pressures shows the maximum difference to be approximately $20 \mathrm{psi}$ at any time during the transient. The steam dome pressure is higher for the TLTA-1 analysis because the $7 \times 7$ bundle has a higher total heat transfer ${ }^{[76]}$ (and thus a higher steam generation rate) than the $8 \times 8$ bundle.

(2) Core Hydrodynamics. The core inlet mass flows calculated for both the $7 \times 7$ and the $8 \times 8$ bundle cases are shown in Figure 97. The core inlet flow drops

[a] RELAP4 EXP*P4/CE 06/25/75 - an experimental version of the code very similar to RELAP4/MOD5 Update 2. 


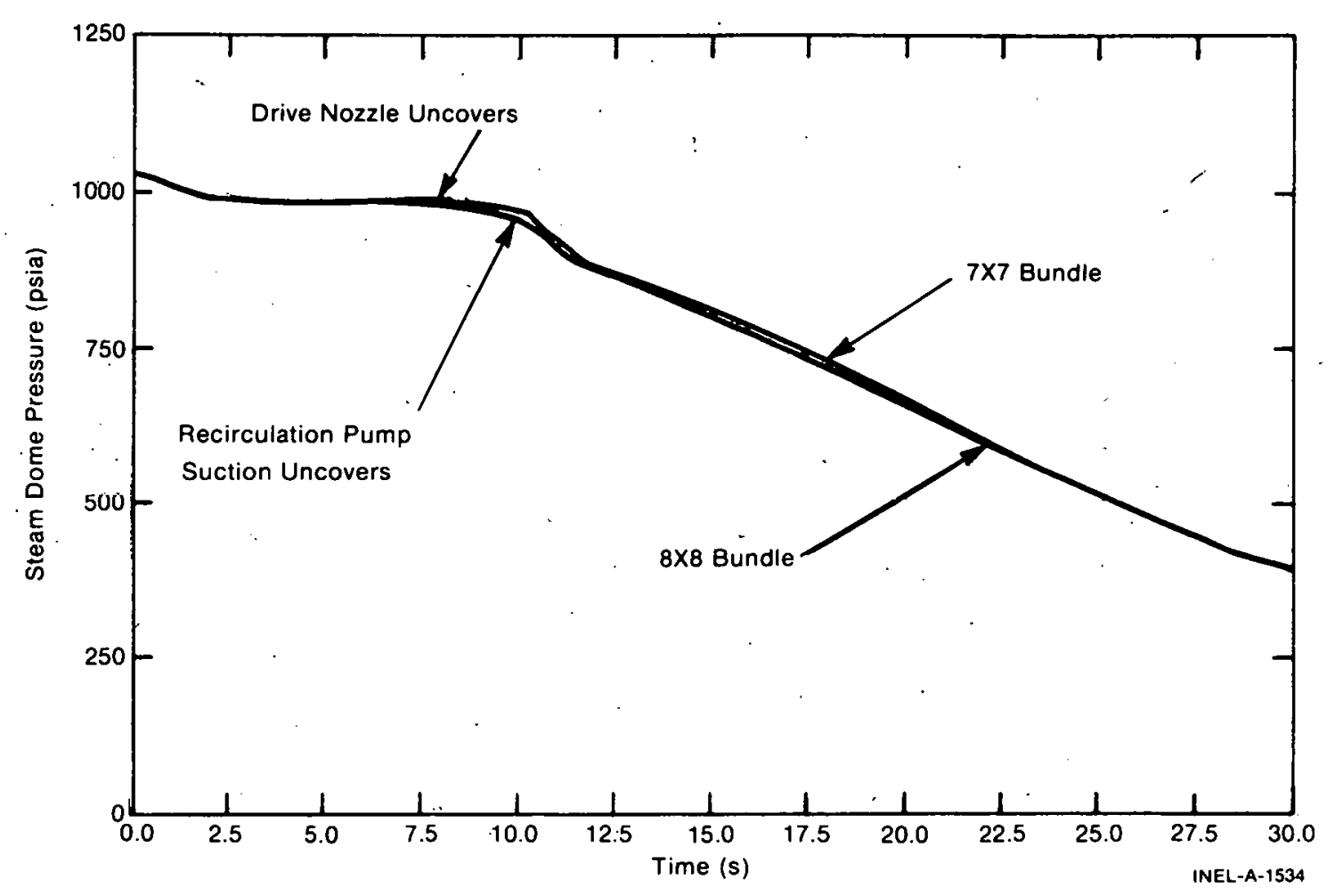

Fig. 96 Comparison of steam dome pressure in TLTA-1 $7 \times 7$ bundle and TLTA-2 $8 \times 8$ bundle cases.

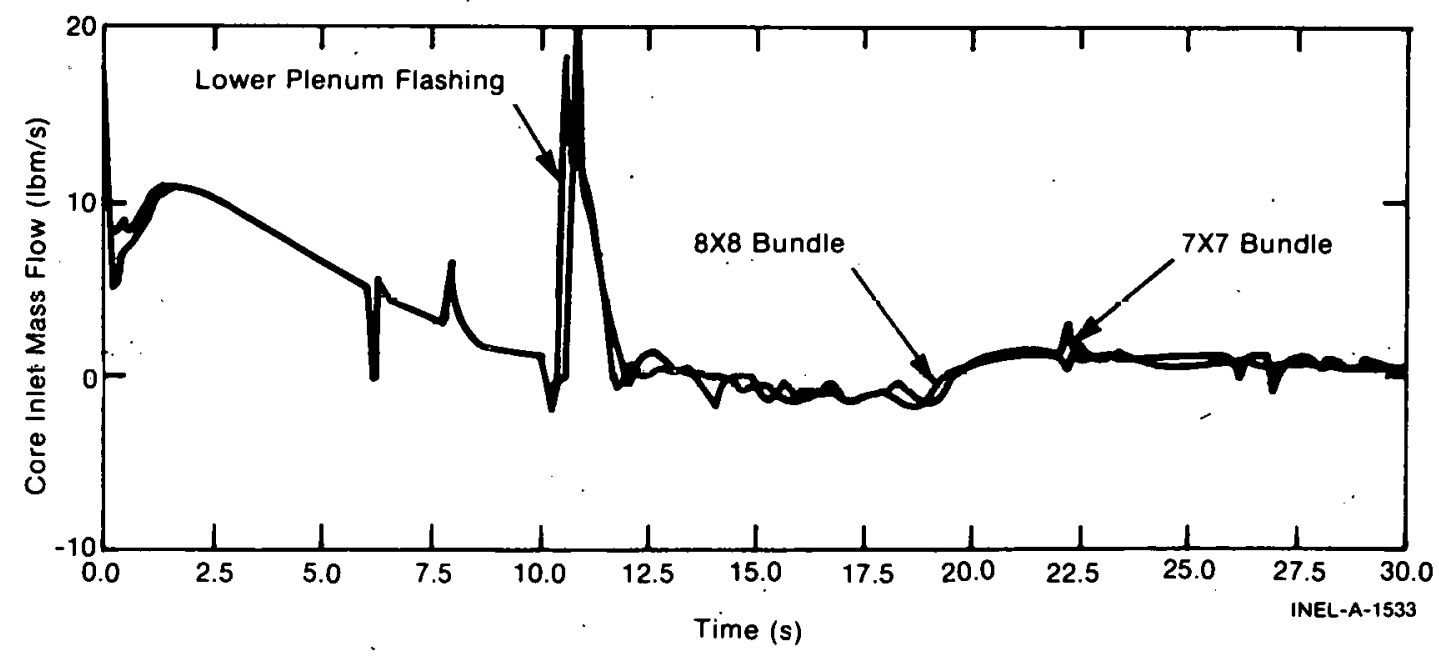

Fig. 97 Comparison of core inlet mass flow in TLTA-1 $7 \times 7$ bundle and TLTA-2 $8 \times 8$ bundle cases. 
immediately following the break to approximately one-third its initial value in both cases. The mass flow continues its downward trend until lower plenum flashing results in a peak value of approximately $19 \mathrm{lbm} / \mathrm{s}$. The lower plenum flow spike lasts about $1.5 \mathrm{~s}$, after which the inlet core flow remains near zero.

Comparison of the core inlet mass flows shows the results to be very similar. The lower plenum flashing occurs $0.3 \mathrm{~s}$ later in the $7 \times 7$ case than in the $8 \times 8$ case. This delay results from the difference in pressure between the two systems at the time of the recirculation pump suction line uncovery.

(3) Core Heat Transfer Characteristics. Figure 98 compares the peak temperature predictions of the $8 \times 8$ hot rod (peaking factor 1.04) at the 96.5 -in. elevation and the $7 \times 7$ hot rod (peaking factor 1.24) at the 98 -in. elevation. The $8 \times 8$ hot rod remains in nucleate boiling for $15 \mathrm{~s}$ and reaches a temperature of $695^{\circ} \mathrm{F}$ at $30 \mathrm{~s}$.

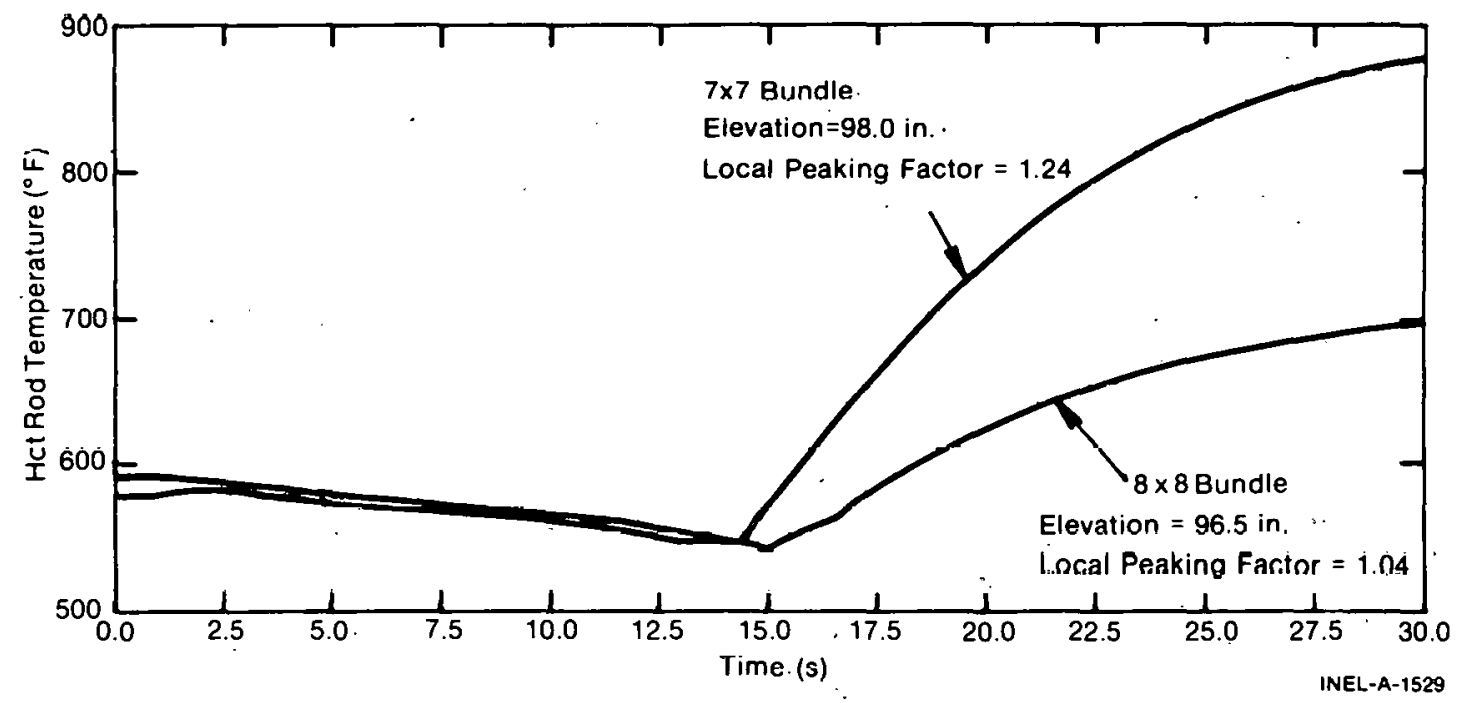

Fig. 98 Comparison of rod temperatures of TLTA-1 $7 \times 7$ bundle and TLTA-2 $8 \times 8$ bundle cases.

The $7 \times 7$ hot rod dries out $0.6 \mathrm{~s}$ earlier than the $8 \times 8$ hot rod and reaches a temperature $180^{\circ} \mathrm{F}$ higher than the $8 \times 8$ hot rod. The $180^{\circ} \mathrm{F}$ temperature difference is primarily a result of the large difference between $8 \times 8$ and $7 \times 7$ local peaking factors and available stored energy.

3.3.3 Conclusions. The peak temperatures measured on the $8 \times 8$ bundle heater rods will be lower than comparable measurements on $7 \times 7$ bundle heater rods for the same test conditions. The TLTA system behavior (hydrodynamics and pressure response) will be much the same with either the $7 \times 7$ or the $8 \times 8$ bundles for the same test conditions. 


\title{
4. BEST ESTIMATE/EVALUATION MODEL COMPARISON
}

\author{
G. W. Johnsen
}

The quantitative differences between best estimate and evaluation model analyses of a postulated loss-of-coolant accident can be used to assess the conservatisms embodied in a reactor licensing calculation. The purpose of the BE/EM study was to make a direct comparison of such analyses, maintaining commonality where possible, so that the quantitative effect of those differences necessitated by the conservatisms of a reactor licensing analysis could be assessed. The best estimate analysis was obtained by applying the currently accepted best judgment mathematical models to a postulated LOCA occurring in a PWR operating in its most probable state. In contrast, the evaluation model analysis was based on conservative models, assuming worst-case PWR operating conditions and the worst single protective system failure.

The evaluation model analysis was conducted in general conformance with the rules specified in the Code of Federal Regulations (10 CFR 50, Appendix $\mathrm{K}^{[77]}$ ). However, it was not a licensing analysis as defined by Appendix $\mathrm{K}$, which would require approved computer codes (RELAP4/MOD5[73] is not approved for licensing) and a spectrum of analyses to determine the limiting case in terms of break area, discharge coefficient, nodalization, etc. Nevertheless, the evaluation model calculation embodied the basic requirements set forth in the rule.

The LOCA chosen for the study was a double-ended cold leg guillotine break. While a highly unlikely event, this is generally a limiting condition for plant licensing and therefore suitable for comparing best estimate and evaluation model analyses. To enhance the value of the study, an operational plant, the ZION I PWR [a], was chosen for analysis. This allowed the use of available plant operating data rather than relying solely on design specifications. The analyses considered the LOCA from rupture to beginning of reflood. This limitation was placed on the study due to the lack of a fully developed best astimate reflood code. However, the intent is to extend the study to consider reflood when RELAP4/MOD6 is available.

Differences between the best estimate and evaluation model analyses were in three aręas: (a) plant operating condition at accident initiation (Table XXI), (b) event sequence during the accident (Table XXII), and (c) the codes and mathematical models applied (Figure 99).

In the best estimate analysis, the plant was assumed to be operating nominally at rated power at the time of the accident. The core was assumed to be in an equilibrium state and.

[a] The ZION 1 reactor is a four-loop PWR that contains fuel bundles having a $15 \times 15$ rod array. The reactor was designed by the Westinghouse Electric Corporation and is owned and operated by the Commonwealth Edison Company. 
TABLE XXI

INITIAL PLANT OPERATING CONDITIONS

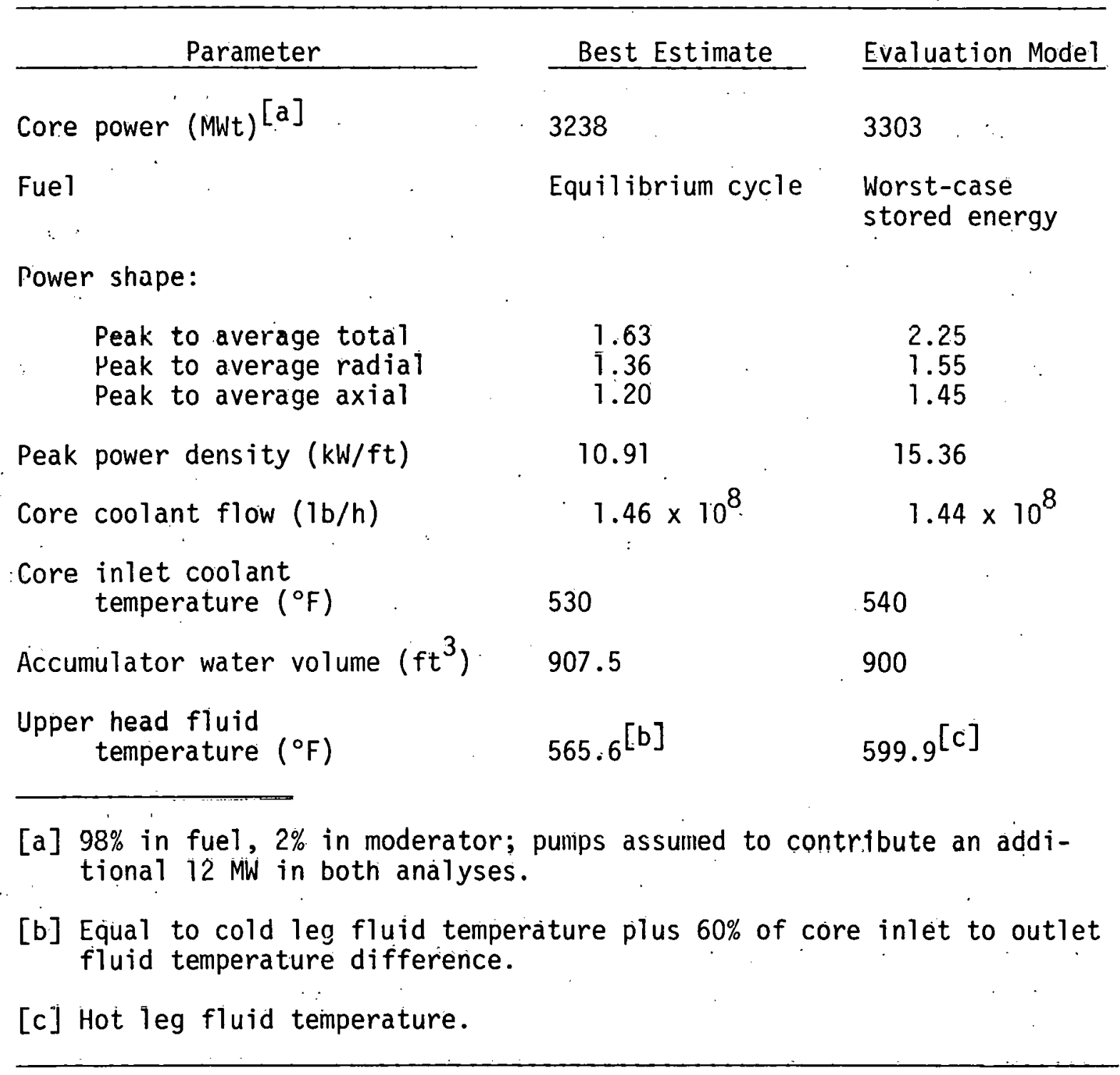




\section{TABLE XXII}

\section{ASSUMPTIONS FOR LOCA SEQUENCE}

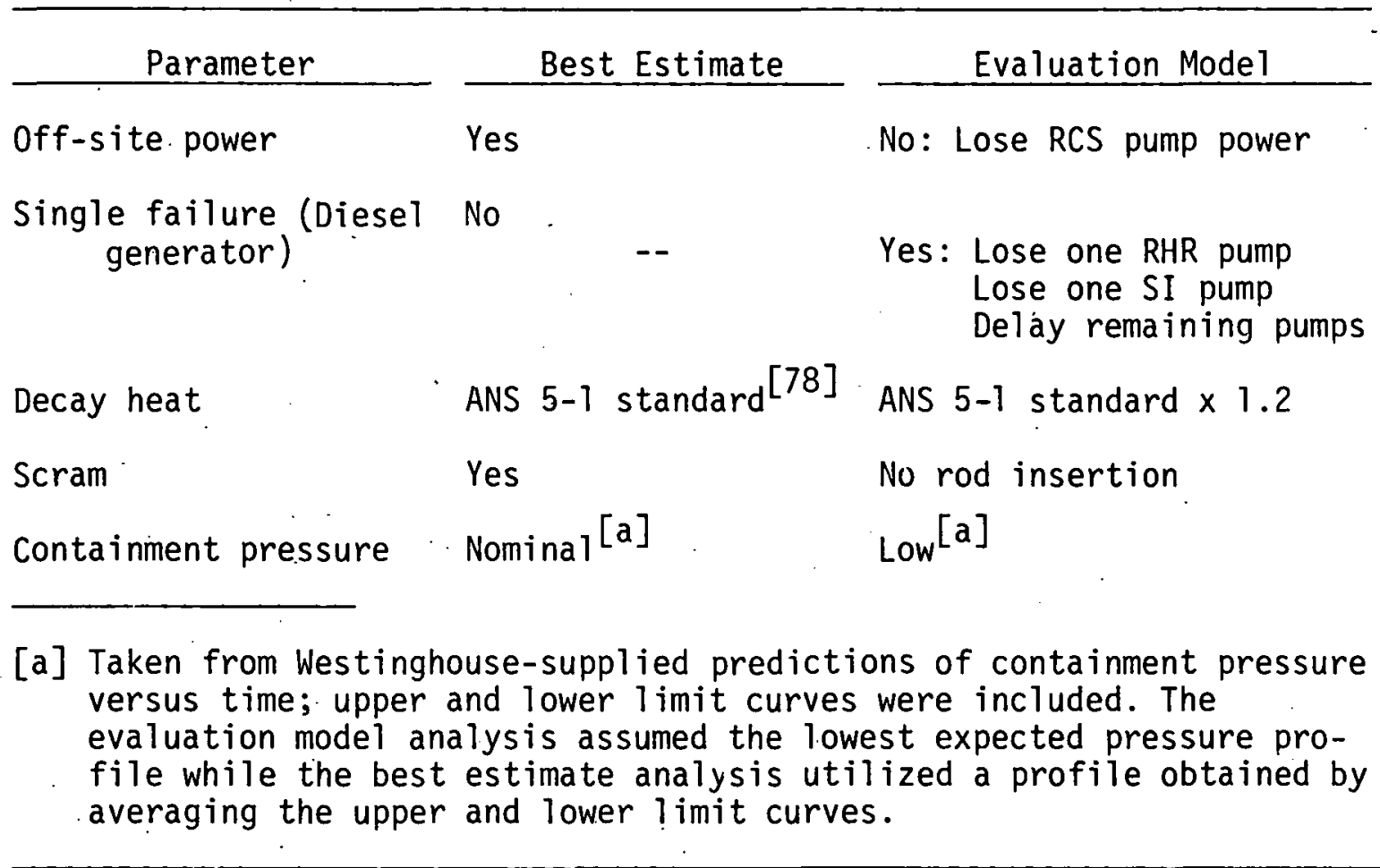

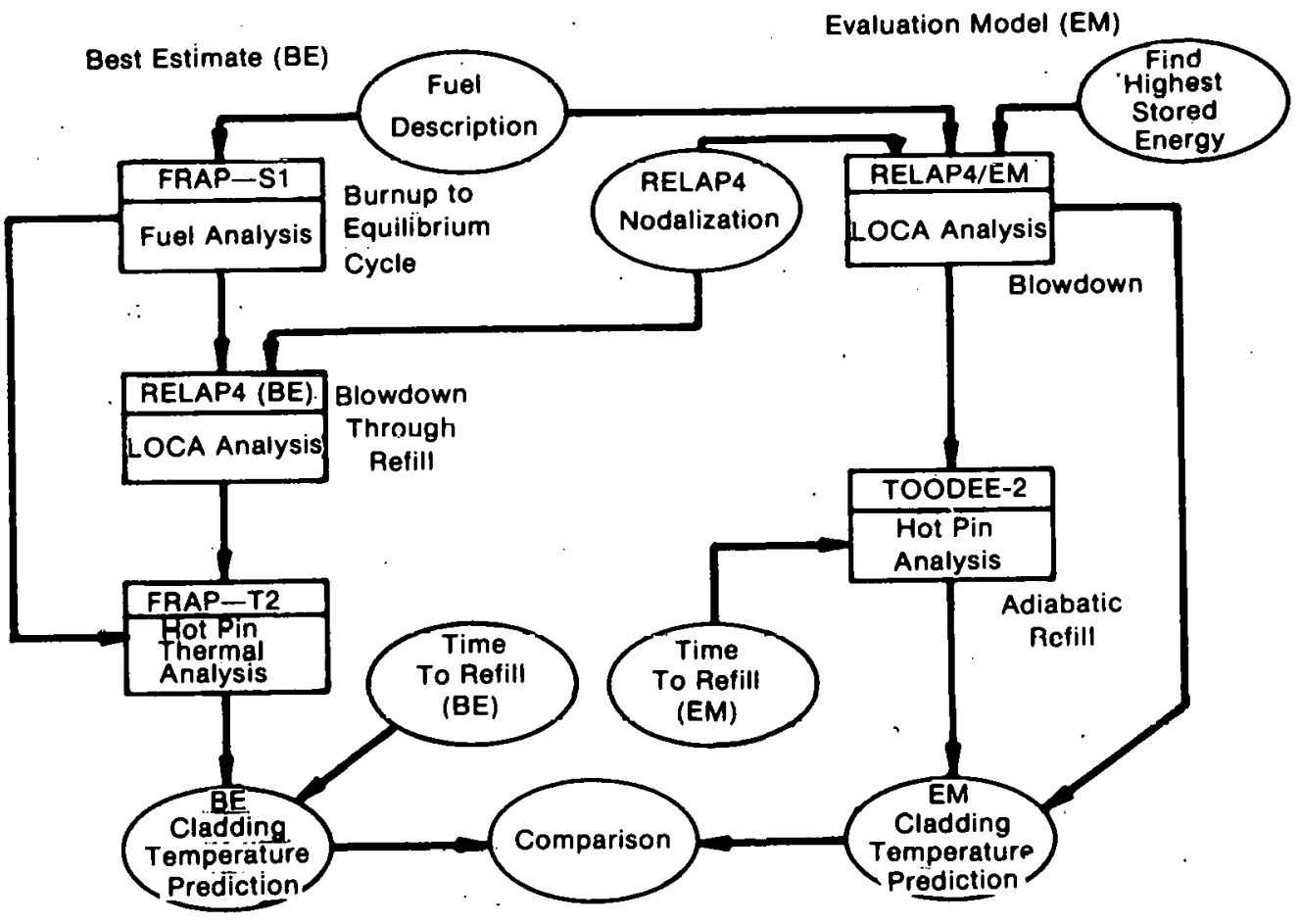

Fig. 99 Analy tical modcls and analy sis process.

INEL-A-1532 
all safety systems remain operational throughout the transient. The mathematical models applied were those considered most representative of anticipated behavior. The bestestimate analysis consisted of a three-step process: (a) defining the fuel characteristics at equilibrium cycle utilizing the FRAP-S1 code; (b) conducting the thermal-hydraulic transient analysis with RELAP4/MOD-E, Version 73, updated for BE/EM; and (c) performing a detailed hot pin thermal analysis with the FRAP-T2 code using the boundary conditions from FRAP-S1 and RELAP4.

The evaluation model analysis assumptions included worst case fuel burnup (highest stored energy), a $2 \%$ overpower condition, loss of offsite power, and the single worst failure in the plant emergency protective system. The special models used in the evaluation model calculations incorporate conservatisms consistent with 10 CFR 50, Appendix K, and in gencral contormed to the rules specified in the Water Reactor Evaluation Model manual[63]. The evaluation model analysis consisted of linking the results from RELAP4/MOD-E, Version 73, updated for BE/EM, with a TOODEE-2[79] hot channel calculation. The RELAP4 evaluation model analysis included a prediction of the hot pin behavior within the hot assembly from initiation of blowdown to end-of-bypass. The fuel rod thermal and mechanical characteristics at end-of-bypass were input to the TOODEE-2 code, which computed hot pin behavior to the beginning of reflood, assuming an adiabatic heatup.

Figure 100 shows the results of the best estimate and evaluation model comparison with respect to peak cladding temperature. Two best estimate calculations were made, differing only in the method employed to compute critical flow at the side of the pipe break

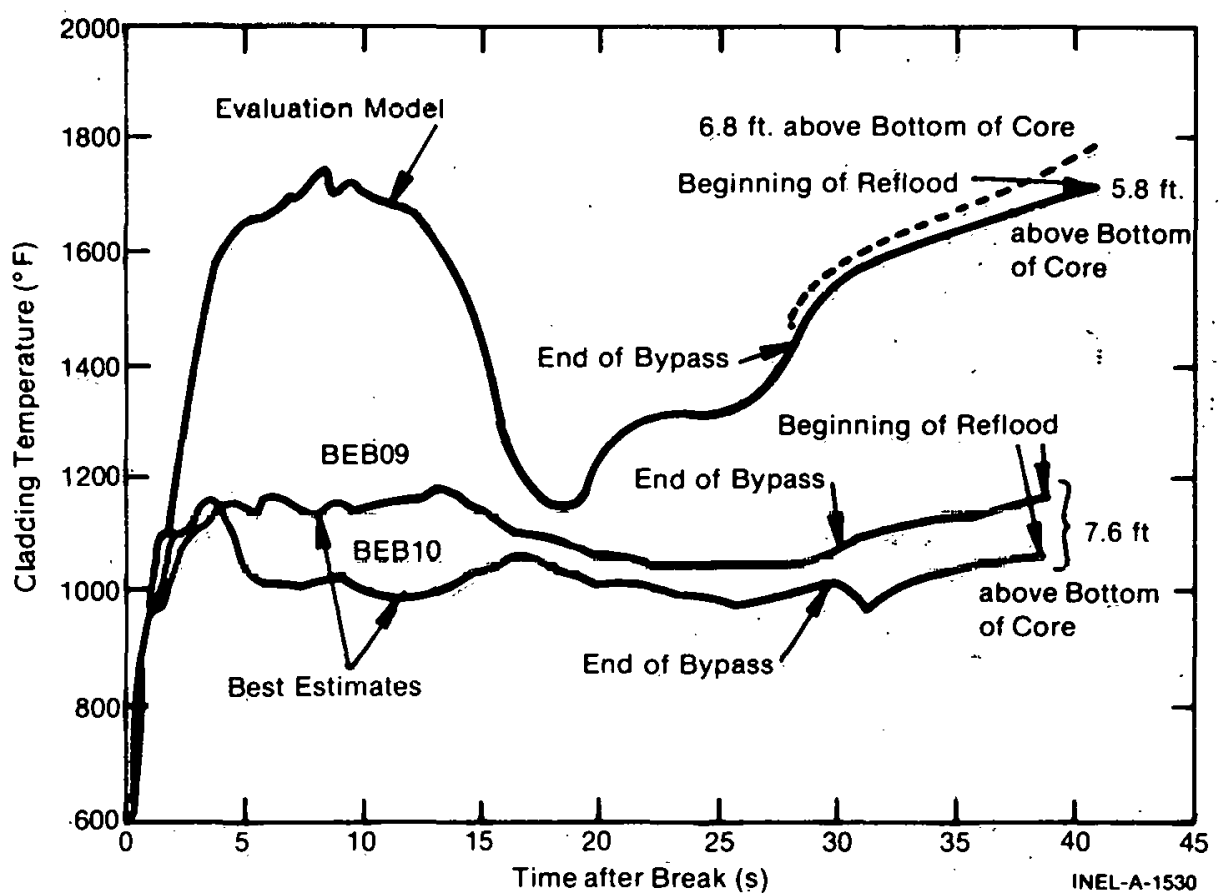

Fig. 100 Comparison of hot spot cladding temperature, evaluation model versus best estimates. 
nearest the vessel[a]. As indicated, the evaluation model peak cladding temperature exceeds that of the best estimate analyses by $600^{\circ} \mathrm{F}$. Furthermore, while clad rupture was predicted to occur during refill in the evaluation model analysis, no swelling or rod deformation was calculated to occur in the best estimate analysis.

[a] Best Estimate Case BEB09 and the evaluation model case used approximate local stagnation fluid properties to compute critical flow; Case BEB10 used static fluid properties. The two calculations indicate the differences between the technically correct method (Case BEB09) and the method which has been historically used (Case BEB10). 


\section{REFERENCES}

1. E. M. Feldman and D. J. Olson, Semiscale Mod-1 Program and System Description for the Blowdown Heat Transfer Tests (Test Series 2), ANCR-1230 (August 1975).

2. E. M. Feldman, B. L. Collins, K. E. Sackett, Experiment Data Report for Semiscale Mod-1 Test S-05-2 (Alternate ECC Injection Test), TREE-NUREG-1051 (February 1977).

3. M. L. Patton, Jr., B. L. Collins, K. E. Sackett, Experiment Data Report for Semiscale Mod-1 Tests S-05-2A and S-05-2B (Alternate ECC Injection Tests) TREENUREG-1057 (April 1977):

4. H. S. Crapo, B. L. Collins, K. E. Sackett, Experiment Data Report for Semiscale Mod-1 Iests SS-U4-S and S'U4-6' (Baseline ECC Tests); TREE-NUREG-1045 (January 1977).

5. C: L. Wheeler et al, COBRA-IV-I: An Interim Version of COBRA for ThermalHydraulic Analysis of Rod Bundle Nuclear Fuel Elements and Cores, BNWL-1962 (March 1976):

6. K. A. Dietz (ed.), Quarterly Technical Progress Report on Water Reactor Safety Programs Sponsored by the Nuclear Regulatory Commission's Division of Reactor Safety Research, April - June 1976, TREE-NUREG-1004 (October 1976).

7. R. V. MacBeth, Burnout Analysis Part 3. The Low Velocity Burnout Regime, AEEW-R, 222 (1963).

8. L. Biasi et al, Studies on Burnout, Part 3, Energia Nucleare, Vol. 14, No. S (1967) pp 530-536.

9. P. G. Barnett, A Correlation of Burnout Data for Uniformly Heated Annuli and Its Use for Predicting Burnout in Uniformly Heated Rod Bundles, AEEW-R, 463 (1966).

10. J. S. Gellerstedt et al, Two-Phase Flow and Heat Transfer in Rod Bundles, American Society of Mechanical Engineers, (1969) pp 63-71.

11: L. S. Tong, Boiling Crisis and Critical Heat Flux, TID-25887 (1972).

12. G. M. Roy, "Getting More out of BWR's", Nucleonics Vol. 24, No. 11 (1966).

13. K. A. Dietz (ed.), Quarterly Technical Progress Report on the Water Reactor Safety Programs Sponsored by the Nuclear Regulatory Commission's. Division of Reactor Safety Research, July - September 1976, TREE-NUREG-1017 (January 1977). 
14. A. S. Mehner et al, Postirradiation Examination Results for the Irradiation Effects Scoping Test 1, ANCR-NUREG-1336 (September 1976).

15. W. J. Quapp, D. W. Croucher, C. M. Allison, Irradiation Effects Test Series, Test IE-3, Quick Look Report, TFBP-TR-145 (NRC Public Document Room File No. RES-77-27) (November 1976).

16. M. King and L. :C. Farrar, Irradiation Effects Test Series, Test IE-3, Experiment Predictions, TFBP-TR-138 (submitted to NRC Public Document Room) (October 1976).

17. J. A. Dearien et al, FRAP-T2 - A Computer Code for the Transient Analysis of Oxide Fuel Rods, TREE-NUREG-1040 (March 1977).

18. R. W. Garner et al, Gap Conductance Test Series, Test GC 1-3, Test Results Report, TREE-NUREG-1067 (to be published).

19. R. W. Garner and D. T. Sparks, Gap Conductance Test Series, Test GC PCM-3, Quick Look Report, TFBP-TR-130 (NRC Public Document Room File No. REG-76-500) (July 1976).

20. Quarterly Technical Progress Report on Water Reactor Safety Programs Sponsored by the Nuclear Regulatory Commission's Division of Reactor. Safety Research, October - December 1975, ANCR-NUREG-1301 (May 1976).

21. W. R. Smalley, Saxton Plutonium Program: Semi-Annual Progress Report for the Period Ending June 30, 1969, WCAP-3385-20 (October 1969).

22. T. E. Caye and W. R. Smalley, Saxton Plutonium Project, Quarterly Progress Report for the Period Ending December 31, 1970, WCAP-3385-26 (March 1970).

23. W. R. Smalley, Evaluation of Saxton Core III Fucl Materials Performance, WCAP-3385-57 (July 1974).

24. E. F. Ibrahim, In-Reactor Tubular Creep of Zircaloy-2 at $260^{\circ} \mathrm{C}$ and $300^{\circ} \mathrm{C}$, Journal of Nuclear Materials, 46 (1973) pp 169-182.

25. H. Conrad, "Experimental Evaluation of Creep and Stress-Rupture," in Mechanical Behavior of Materials at Elevated Temperatures, New York: McGraw-Hill Book Company, Inc., 1961, p 149.

26. P. A. Ross-Ross and V. Fidleris, "Design Basis for Creep of Zirconium Alloy Components in a Fast Neutron Flux," International Conference on Creep and Fatigue in Elevated Temperature Applications, Philadelphia and Sheffield, UK, 1973, pp 216.1-216.7. 
27. V. Fidleris, "The Effect of Texture and Strain Aging on Creep of Zircaloy-2," Applications Related Phenomena for Zirconium and Its Alloys ASTM-STP-458, Philadelphia: American Society for Testing and Materials, 1969, pp 1-17.

28. P. H. Kreyns and Burkart, "Radiation-Enhanced Relaxation in Zircaloy-4 and $\mathrm{Zr} / 2.5$ wt\% Nb/0.5 wt\% Cu Alloys," Journal of Nuclear Materials, 26 (1968) pp 87-104.

29. F. A. Nichols, "Theory of the Creep of Zircaloy During Neutron Irradiation, Journal of Nuclear Materials, 30 (1969) pp 249-270.

30. P. A. Ross-Ross and Hunt, "The In-Reactor Creep of Cold-Worked Zircaloy-2 and Zirconium-2.5 wt\% Niobium Pressure Tubes," Joumal of Nuclear Materials, 26 (1968) pp 2-17.

31. D. S. Wood, "Dose Dependence of Irradiation Creep of Zircaloy-2," Properties of Reactor Structural Alloys after Neutron or Particle Irradiation ASTM-STP-570, Philadelphia: American Society for Testing and Materials, 1975, pp 207-217.

32. C. C. Busby and L. S. White, Some High Temperature Mechanical Properties of Internally Pressurized Zircaloy-4 Tubing, WAPD-TM-1243 (February 1976).

33. V. Fidleris and C. D. Williams, "Influence of Neutron Irradiation of Zircaloy-2 at $300^{\circ} \mathrm{C}$," Journal of Electrochemical Technology, 4 (May - June 1966) pp 258-267.

34. P. J. Pankaskie, Irradiation Effects on the Mechanical Properties of Zirconium and Dilute Zirconium Alloys-A Review, BN-SA-618 (July 1976, updated November 1976).

35. C. E. Colcman, "Tcrtiary Crecp in Cold-Worked Zircaloy-2," Journal of Nuclear Materials, 42 (1971) pp 180-190.

36. E. Kohn, "In-Reactor Creep of $\mathrm{Zr}-2.5 \% \mathrm{Nb}$ Fuel Cladding," Symposium on Zirconium in the Nuclear Industry, Quebec City, August 10-12, 19\%.

37. F. J. Azzarto et al, "Unirradiated In-Pile and Post-Irradiation Low Strain Rate Tensile Properties of Zircaloy-4," Journal of Nuclear Materials, 30 (1969) pp 208-218.

38. V. Fidleris, "Uniaxial In-Reactor Creep of Zirconium Alloys," Journal of Nuclear Materials, 26 (1968) pp 51-76.

39. D. S. Wood and B. Watkins, " $\Lambda$ Crecp Limit $\Lambda$ pproach to tho Dosign of Zircaloy 2 Reactor Pressure Tubes at $275^{\circ} \mathrm{C}$," Journal of Nuclear Materials, 41 (1971) pp 327-340. 
40. M. Bernstein, "Diffusion Creep in Zirconium and Certain Zirconium Alloys," Transactions of the Metallurgical Society of AIME, 239 (1969) pp 1518-1522.

41. F. A. Nichols, "On the Mechanisms of Irradiation Creep in Zirconium-Base Alloys," Journal of Nuclear Materials, 37 (1970) pp 59-70.

42. V. Fidleris, "Uniaxial In-Reactor Creep of Zirconium Alloys," Journal of Nuclear Materials, 26 (1967) pp 51-76.

43. W. J. Duffin and F. A. Nichols, "The Effect of Irradiation on Diffusion-Controlled Creep Processes," Journal of Nuclear Materials, 45 (1972/1973) pp 302-316.

44. G. R. Piercy, "Mechanisms for the In-Reactor Creep of Zirconium Alloys," Journal of Nuclear Materials, 26 (1968) pp 18-50.

45. E. R. Gilbert, "In-Reactor Creep of Reactor Materials," Reactor Technology, 14 (Fall 1971) pp 258-285.

46. M. Gartner and H. Stehle, "In-Pile Creep Behavior of Zircaloy-4 Cladding Tubes at $400^{\circ} \mathrm{C}$, "Siemans Aktiengesellschaft Reaktortechnik: (September 1972).

47. Quarterly Technical Progress Report on Water Reactor Safety Programs Sponsored by the Nuclear Regulatory Commission's Division of Reactor Safety Research, July September 1975, ANCR-1 296 (February 1976).

48. C. O. Hobson and P. L. Rittenhouse, Deformation and Rupture Behavior of Light Water Reactor Fuel Cladding, ORNL-4727 (October 1971).

49. R. G. Sachs, Light-Water-Reactor Safety Research Program: Quarterly Progress Report July - September 1975, ANL-75-72.

50. R. G. Sachs, Light-Water-Reactor Safety Research Program: Quarterly Progress Report October - December 1975, ANL-76-15.

51. R. G. Sachs, Light-Water-Reactor Safety Research Program: Quarterly Progress Report January - March 1976, ANL-76-49.

52. R. G. Sachs, Light-Water-Reactor Safety Research Program: Quarterly Progress Report, April - June 1976, ANL-76-87.

53. Quarterly Technical Progress Report on Water Reactor Safety Programs Sponsored by the Nuclear Regulatory Commission's Division of Reactor Safety Research, January - March 1975, ANCR-1254 (September 1975). 
54. W. Gulden, "Dokumentation von SSYST - Mod-1: Ein Programmsystem zur Berechnung des Brennstabverhaltens bei einem Kuhlmittelverlustunfall," Kernforschungszentrum Karlsruhe Projekt Nukleare Sicherheit - Arbeitsberieht Nr. 58/75 IKE-Berieht Nr. 4-51 (September 1975).

55. J. A. Dearien et al, FRAP-T: A Computer. Code for the Transient Analysis of Oxide Fuel Rods - Volume 1 - Analytical Models and Input Manual, Internal Report I-309-3-53.1 (NRC Public Document Room File No. REG-76-736) (July 1975).

56. P. R. Bevington, Data Reduction and Error Analysis for the Physical Sciences, New York: McGraw-Hill Book Company, 1969.

57. J. Hahn and S. Shapiro, Statistical Models in Engineering, New York: John Wiley and Sons, Inc. 1967.

58. D. R. Coleman and E. T. Laats, FRAP-T: A Computer Code for Transient Analysis of Oxide Fuel Rods - Volume III - FRAP-T2 Model Verification Report, Internal Report SRD-68-76 (NRC Public Document Room File No. REG-76-559) (August 1976).

59. P. E. MacDonald and L. B. Thompson (eds.), MATPRO: A Handbook of Materials Properties For Use in the Analysis of Light Water Reactor Fuel Rod Behavior, ANCR-1263 (February 1976).

60. K. R. Merckx, BULGEX: A Computer Code to Determine the Deformation and the Onset of Bulging of Zircaloy Fuel Rod Cladding, XN-74-27 (A) (December 1974).

61. R. H. Chapman, Multirod Burst Test Program Quarterly Progress Report for January - March 1976, ORNL/NUREG/TM-36 (August 1976).

62. P. L. Rittenhouse et al, The Effect of Light-Water Reactor Fuel Rod Failure on the Area Available for Emergency Coolant Flow Following a Loss-of-Coolant Accident, ORNL-4752 (January 1972).

63. WREM: Water Reactor Evaluation Model (Revision 1); Division of Technical Review, Nuclear Kegulatory ('ommission, NUREG-75/056 (May 1975).

64. J. G. Collier, Convective Boiling and Condensation, London: McGraw-Hill Book Company, Inc. (1972) p 80.

65. D. A. Steen and G. B. Wallis, The Transition from Annular to Annular-Mist Co-Current Two Phase Downflow, Dartmouth College, Interim Report NYO-31 14-2, (June 1964), and Mist Co-Current Two Phase Downflow, Dartmouth College, Interim Report NYO-3114-3, (June 1964). 
66. W. L. Kirchner, Reflood Heat Transfer In A Light Water Reactor, unpublished PhD dissertation, Massachusetts Institute of Technology (January 1976).

67. J. K. Bradie and A. N. Dickson, "Removal of Entrained Liquid Droplets by Wire-Mesh Demisters," Proceedings Instr. Mechanical Engineers Vol. 184, (1969-1970) pp 195-203.

68. J. F. Wilson, "Steam Volume Fraction in a Bubbling Two Phase Mixture," Transactions of the American Nuclear Society (April 1961) pp 356-357.

69. J. F. Wilson, R. J. Grenda, J. F: Patterson, "The Velocity of Rising Steam in a Bubbling Two Phase Mixture," Transactions of the American Nuclear Society (May 1962).

70. B. M. Dunn, G. D. Morgan, L. R. Carter, Multinode Analysis of Core Flooding Line Break for B\&W $2568 \mathrm{MWt}$ Internals Vent Valve Plants, BAW-10064, Topical Report (April 1973).

71. B. C. Slifer and J. E. Hench, Loss-of-Coolant Accident and Emergency Core Cooling Models for General Electric Boiling Water Reactors, NEDO-10329 (April 1971).

72. G. B. Wallis, One-dimensional Two-phase Flow, New York: McGraw-Hill Book Company, Inc., 1969, p 336.

73. RELAP4/MOD5: A Computer Program for Transient Thermal-Hydraulic Analysis of Nuclear Reactors and Related Systems User's Manual, Volume I RELAP4/MODS Description, ANCR-NUREG-1335 (September 1976).

74. Project Description ORNL PWR Blowdown Heat Transfer Separate Effects Program - Thermal-Hydraulic Test Facility (THTF), ORNL/NUREG/TM-2, (February 1976).

75. R. J. Muzzy, Preliminary BWR Blowdown/Emergency Core Cooling Program Plan, GEAP-21 255 (June 1976).

76. J. P. Dougherty and .. R. J. Muzzy, BWR $8 \times 8^{\circ}$ Fuel Rod Simulation Using Electrical Heulers, GEAP-21207 (March 1976).

77. 10 CFR, Part. 50, "Acceptance Criteria for Emergency Core Cooling System for Light-Water-Cooled Nuclear Power .Plants," Federal Register, Vol. 39, No. 3 (January 1974).

78. Proposed ANS Standard Decay Energy Release Rates Following Shutdown of Uranium-Fueled Thermal Reactors, ANS 5-1 (October 1971). 
79. G. N. Lauben, TOODEE 2: A Two-Dimensional Time Dependent Fuel Element Thermal Analysis Program, NUREG-75/057 (May 1975). 


\section{DISTRIBUTION RECORD FOR TREE-NUREG-1070}

Internal Distribution

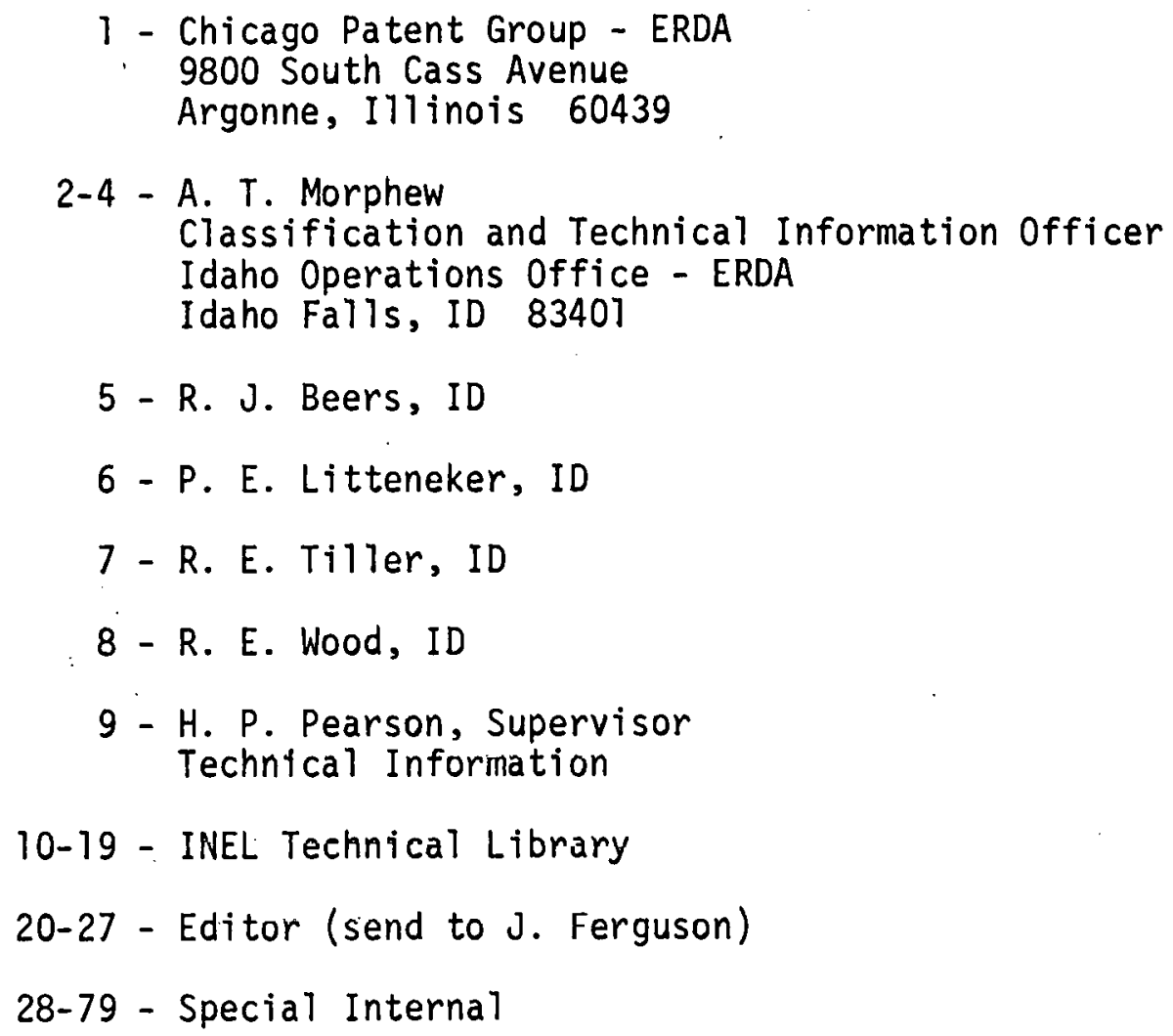

External Distribution

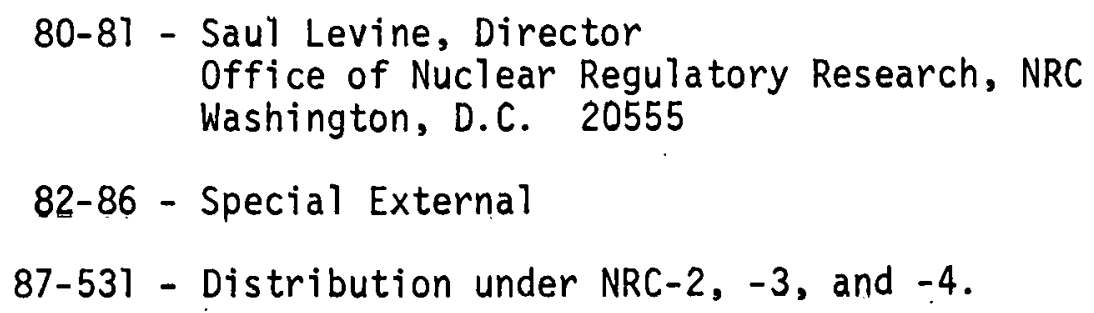

